\title{
Disentangling interfaces and bulk in spin transport calculations
}

Kriti Gupta 
DISENTANGLING INTERFACES AND BULK IN SPIN TRANSPORT CALCULATIONS

Kriti Gupta 
This dissertation has been approved by:

Prof. dr. P.J. Kelly (promotor)
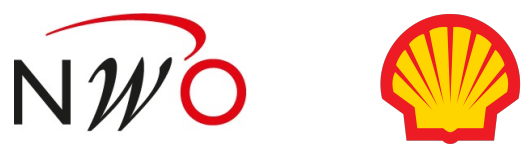

The work in this thesis was carried out in the Computational Materials Science group of the Faculty of Science and Technology (TNW) at the University of Twente. This work is part of the Industrial Partnership Programme (IPP) "Computational Sciences for Energy Research" (CSER) of the Foundation for Fundamental Research on Matter (FOM), which is part of the Netherlands Organisation for Scientific Research (NWO). This research programme is co-financed by Shell Global Solutions International B.V.

Cover design: An artist's impression of spin transport. Magnetic top with LEDs attached spinning on a reflective surface photographed using long exposure. By Sander Wildeman and Kriti Gupta.

Printed by: Gildeprint, Enschede, The Netherlands

Typeset: $\mathrm{BT}_{\mathrm{E}} \mathrm{X}$

ISBN: 978-90-365-4776-5

DOI: $10.3990 / 1.9789036547765$

URL: https://doi.org/10.3990/1.9789036547765

(C)2019, Kriti Gupta, The Netherlands.

All rights reserved. No parts of this thesis may be reproduced, stored in a retrieval system or transmitted in any form or by any means without permission of the author. 


\section{DISENTANGLING INTERFACES AND BULK IN SPIN TRANSPORT CALCULATIONS}

\section{DISSERTATION}

to obtain

the degree of doctor at the University of Twente, on the authority of the rector magnificus, Prof. dr. T.T.M. Palstra, on account of the decision of the Doctorate Board, to be publicly defended on Friday the $24^{\text {th }}$ of May 2019 at 16:45 hrs.

$$
\text { by }
$$

Kriti Gupta

born on the $18^{\text {th }}$ of December, 1990

in Bhopal, India 
Graduation Committee:

Chairman: Prof. dr. J.L. Herek

Secretary: Prof. dr. J.L. Herek University of Twente, TNW

Supervisor: Prof. dr. P.J. Kelly University of Twente, TNW

Members: Prof. dr. ir. W.G. van der Wiel University of Twente, EWI Prof. dr. ir. A. Brinkman University of Twente, TNW Prof. dr. G.E.W. Bauer Tohoku University

Prof. dr. S.T.B. Goennenwein Technische Universität Dresden Prof. dr. Zhe Yuan Beijing Normal University 


\section{Contents}

1 Introduction $\quad 1$

1.1 Origins of spin transport $\ldots \ldots \ldots \ldots \ldots \ldots \ldots$

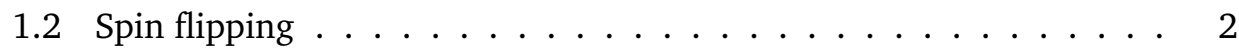

1.3 Spin Hall effect $\ldots \ldots \ldots \ldots \ldots \ldots$

1.4 Scope of this thesis $\ldots \ldots \ldots \ldots \ldots \ldots \ldots$

1.5 Computational scheme . . . . . . . . . . . . . 8

1.5.1 Density functional theory . . . . . . . . . . . 8

1.5.2 Solving the Kohn-Sham Schrödinger equation for the two terminal geometry . . . . . . . . . . . . 9

1.5.3 Self-consistent atomic potentials: Linearized muffin-tin orbital basis . . . . . . . . . . . . 10

1.5.4 Practical implementation . . . . . . . . . . . . 12

2 Calculating spin transport properties $\quad 15$

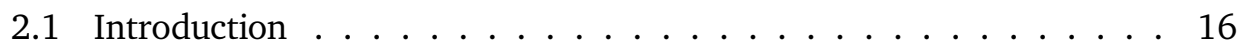

2.2 Methods . . . . . . . . . . . . . . . . 20

2.2.1 Semiclassical transport theory . . . . . . . . . . . . 20

2.2.2 Quantum Mechanical Scattering . . . . . . . . . . 22

2.2.3 Calculating the full current tensor . . . . . . . . . . 23

2.2.4 Layer averaged current tensor . . . . . . . . . . . . . . 26

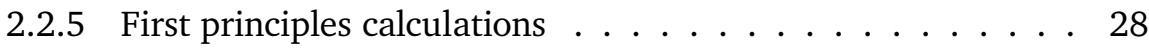

2.3 Results . . . . . . . . . . . . . . . . . 32

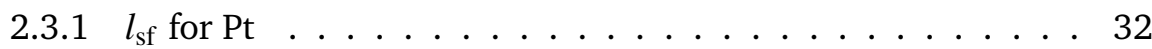

$2.3 .2 \beta$ for Permalloy . . . . . . . . . . . . . . . . 38

$2.3 .3 l_{\mathrm{sf}}$ for Permalloy $\ldots \ldots \ldots \ldots \ldots$

2.3.4 Spin Hall angle for Platinum _. . . . . . . . . . . . 42

2.4 Comparison with other work . . . . . . . . . . . . . 46

2.5 Summary and Conclusions . . . . . . . . . . . . . 51

3 Calculating interface transport parameters at finite temperatures: Nonmagnetic interfaces 53

3.1 Introduction $\ldots \ldots \ldots \ldots \ldots \ldots \ldots \ldots \ldots$

3.2 Methods . . . . . . . . . . . . . . . 56 
3.2.1 Valet-Fert model . . . . . . . . . . . . . . . 56

3.2 .2 Interface discontinuity . . . . . . . . . . . . . 57

3.2.3 Transverse spin current at an interface . . . . . . . . . . 60

3.2.4 Transverse charge current and interface SHE . . . . . . . . . 61

3.3 Calculations . . . . . . . . . . . . . . . . . 62

3.4 Results . . . . . . . . . . . . . . . . . . . . . . 64

3.4.1 AulPt: interface resistance . . . . . . . . . . . . . . . 64

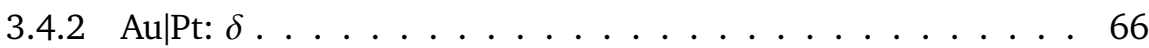

$3.4 .3 \mathrm{AulPt}$ interface SHE . . . . . . . . . . . . . . . . 68

$3.4 .4 \mathrm{AulPt}$ interface ISHE - $\Theta_{\mathrm{I}} \ldots \ldots \ldots$. . . . . . . . . 68

$3.4 .5 \mathrm{Au} \mid \mathrm{Pt}$ vs $\mathrm{Au} \mid \mathrm{Pd} \ldots \ldots \ldots \ldots$. . . . . . . . . . . . . 71

3.4.6 Temperature dependence of the interface parameters . . . 72

3.5 Summary and Conclusions . . . . . . . . . . . . . . . 72

4 Calculating interface transport parameters at finite temperatures: Ferromagnetic | Nonmagnetic interfaces $\quad 77$

4.1 Introduction . . . . . . . . . . . . . . . . . . 78

4.2 Methods . . . . . . . . . . . . . . . . 80

4.2.1 Valet-Fert model . . . . . . . . . . . . . . . . 80

4.2 .2 Interface discontinuity . . . . . . . . . . . . 83

4.3 Calculations . . . . . . . . . . . . . . . 86

4.4 Results and Discussion . . . . . . . . . . . . . . . . . . . . 90

4.4.1 Bulk materials . . . . . . . . . . . . . . . . 90

4.4 .2 Interfaces . . . . . . . . . . . . . . . . . . 94

4.5 Comparison with other experiments and calculations . . . . . . 100

4.6 Conclusions . . . . . . . . . . . . . . . . . . . . . . 101

5 Intrinsic spin-Hall effect in Permalloy 103

5.1 Introduction . . . . . . . . . . . . . . . . . . . 104

5.2 Calculations . . . . . . . . . . . . . . . 105

5.3 Results . . . . . . . . . . . . . . . . . 106

5.3.1 Spin-Hall effect in Py at 0 K . . . . . . . . . . . . 106

5.3 .2 Effect of SC size . . . . . . . . . . . . . . . . 109

5.3.3 Spin-Hall effect in Py as a function of temperature . . . . . . 110

5.4 Microscopic origin of the spin Hall effect in Py . . . . . . . . . . . . . . 111

5.5 Summary . . . . . . . . . . . . . . . . . . . . . 114

$\begin{array}{ll}\text { Bibliography } & 115\end{array}$

$\begin{array}{ll}\text { Summary } & 129\end{array}$

$\begin{array}{ll}\text { Acknowledgements } & 133\end{array}$ 


\section{1 \\ Introduction}

\subsection{Origins of spin transport}

Our ability to manipulate the transport of electric charge, first through vacuum and then through matter, has developed over little more than a hundred years and has been truly revolutionary. Starting with radio and television based upon vacuum tubes and culminating with modern digital computers, the internet, smartphones etc. electronics has increasingly pervaded our lives. In the past three decades, another fundamental property of the electron, its spin, has emerged as a new dimension for the design of electronic devices. Spin-based electronics, or "spintronics", is already widely applied in read heads for hard disk drives. The operating principle is based upon detecting bits stored in a magnetic medium using spin-dependent electron transport through a layered ferromagnetic structure in the read head.

The scattering probability of an electron travelling through a "strong" ferromagnet like cobalt is low (high) if its spin is parallel (antiparallel) to the magnetization direction [1]. A read head consists of two ferromagnetic layers separated by a nonmagnetic metallic or (more recently) insulating spacer layer. The ease of passage of electrons through this structure depends on the relative orientation of the magnetizations of the two ferromagnetic layers. Unless stated otherwise, we will refer to spins parallel to the magnetization of the uppermost ferromagnet in Fig. 1.1 as "spin-up" and to those antiparallel as "spin-down". When the ferromagnetic layers have parallel magnetic orientations, spin-up electrons undergo minimal scattering in both layers compared to spin-down electrons (illustrated in the left panel of Fig. 1.1). On the other hand, when the magnetizations in the two layers are antiparallel, both spin-up and spin-down electrons are scattered strongly in the layer 

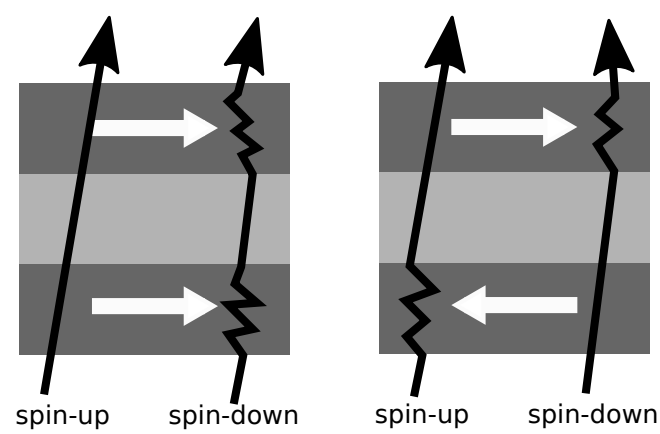

Figure 1.1: Spin dependent scattering in an FM|NM|FM geometry for parallel (lefthand side) and antiparallel (rhs) alignment of the magnetic layers.

where their spin is antiparallel to the magnetization (right panel of Fig. 1.1). The antiparallel configuration leads to an electrical resistance that is much higher than that of the parallel configuration. This large difference in resistances is referred to as "giant" magnetoresistance (GMR).

\subsection{Spin flipping}

GMR was discovered in 1988 for a Fe|Cr|Fe trilayer [2, 3] and the discovery was recognized with the award of the Nobel prize for physics in 2007. The observed difference between antiparallel and parallel resistances was 50\%. This discovery was groundbreaking because it highlighted how significantly electron transport can depend on the electron spin. The early models used to analyze GMR [4, 5] assumed that the spin-up and spin-down electrons traverse the device independently, acting as two independent channels that together determine the total conduction of the device. This assumption is only valid if the layer thicknesses for both ferromagnetic and nonmagnetic materials are much smaller than the length scale over which the electron spins can flip (the so called spin-flip diffusion length, $l_{\text {sf }}$ ). The early models ignored spin-flip scattering in the layers but noted that there were contributions to the spin-dependent scattering from the bulk of the layers as well as from the interfaces between the layers.

The configuration of the first GMR devices was such that Current flowed In the Plane (CIP) of the layers. Soon, GMR studies were extended to geometries where the Current was Perpendicular to the layer Planes (CPP) [6, 7]. Because of their higher (axial) symmetry, CPP geometries are more amenable to theoretical analysis and better suited for the study of spin transport through multilayers on length scales comparable to $l_{\mathrm{sf}}$. Before the discovery of GMR, spin-flipping in a CPP ferromagnetic|nonmagnetic (FM|NM) bilayer had already been studied theoretically using a diffusion model [8]. This model, illustrated in Fig. 1.2, describes 


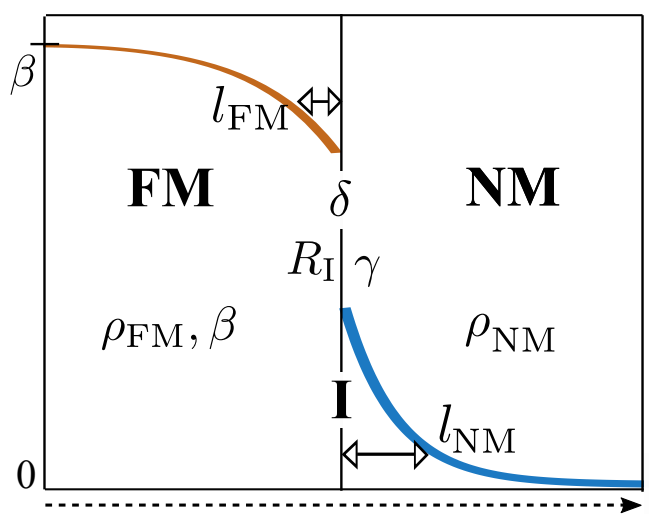

Charge current

Figure 1.2: Spin current through a ferromagnetic|nonmagnetic (FM|NM) bilayer as described by the diffusion model $[8,9]$. The length scale over which spin current equilibriates in the FM and NM materials is the spin flip diffusion length $l_{\mathrm{FM}}$ and $l_{\mathrm{NM}}$ respectively. In a FM material, a spin current is additionally characterized by the spin-dependent resistivities $\rho_{\uparrow}$ and $\rho_{\downarrow}$ or equivalently the total bulk resistivity $\rho_{\mathrm{FM}}$ and spin-asymmetry parameter $\beta=\left(\rho_{\downarrow}-\rho_{\uparrow}\right) /\left(\rho_{\downarrow}+\rho_{\uparrow}\right)$. In the nonmagnetic material the bulk resistivity is $\rho_{\mathrm{NM}}$ and $\beta=0$. The interface is characterized in terms of spin-dependent interface resistances $R_{\uparrow}$ and $R_{\downarrow}$ or equivalently the total interface resistance $R_{\mathrm{I}}$ and interface spin-asymmetry parameter $\gamma$. Spin-flip scattering at the interface leads to a discontinuity in the spin current and is characterized by the spin memory loss parameter $\delta$.

the flow of a charge current through the bilayer as follows. The polarization of the charge current far from the interface is $\beta=\left(\rho_{\downarrow}-\rho_{\uparrow}\right) /\left(\rho_{\downarrow}+\rho_{\uparrow}\right)$ where $\rho_{\uparrow}$ and $\rho_{\downarrow}$ denote the resistivities for spin-up and spin-down electrons in an infinite, bulk sample of either material. In a nonmagnetic material there is symmetry between spin-up and spin-down electrons, $\rho_{\uparrow}=\rho_{\downarrow}$ and $\beta=0$; a charge current is unpolarized far to the right of the interface in Fig. 1.2. However, in the vicinity of the interface, the finite spin-polarized current from the ferromagnetic layer leads to an "accumulation" of spin. This build-up is balanced over the length scale $l_{\text {sf }}$ unique to each metal. A spin current injected into a nonmagnetic material much thicker than its $l_{\mathrm{NM}} \equiv l_{\mathrm{sf}}^{\mathrm{NM}}$ will decay completely to zero. Likewise, a charge current injected from a nonmagnetic material into a ferromagnet much thinner than $l_{\mathrm{FM}} \equiv l_{\mathrm{sf}}^{\mathrm{FM}}$ will not attain the finite polarization value $\beta$. Thus, knowledge of $l_{\mathrm{sf}}$ for both nonmagnetic and ferromagnetic metals is essential to plan device dimensions.

On the same lines as the above, a phenomenological model based upon the semiclassical Boltzmann formalism was proposed by Valet and Fert to analyze CPPGMR experiments [9]. This model took into account spin-dependent scattering in the bulk and at interfaces as well as spin-flip scattering in individual layers by 
including momentum and spin relaxation. They arrived at macroscopic equations that describe the spatial distribution of spin accumulation in multilayers and spin currents through them in terms of a spin-flip diffusion length $l_{\mathrm{sf}}$ and spin-dependent resistivities $\rho_{\downarrow}$ and $\rho_{\uparrow}$ for each metal (with $\rho_{\downarrow}=\rho_{\uparrow}$ for nonmagnetic metals) as well as spin-dependent interface resistances $R_{\uparrow}$ and $R_{\downarrow}$. The Valet-Fert model was adopted almost universally to interpret CPP-GMR experiments and underlies the determination of $l_{\mathrm{sf}}$ in various magnetic and nonmagnetic metals [10].

$l_{\mathrm{sf}}$ is found to span orders of magnitudes from the micron lengthscale for $3 d$ metals such as $\mathrm{Cu}$ [10] to a couple of nanometres for $5 d$ metals such as Pt [11, 12] at room temperature. The spin-flip scattering in pure metals at finite temperatures is dominated by electron-phonons interactions involving intrinsic spin-orbit coupling $[13,14]$. The lowering of symmetry at interfaces leads to a larger effective spinorbit coupling. This results in enhancement of spin-flip scattering at interfaces and a discontinuity in the spin currents. It has been shown that neglecting interface spin-flipping can lead to incorrect estimates of $l_{\mathrm{sf}}$ for heavy transition metals with large spin-orbit coupling, such as Pt [15]. The range of $l_{\mathrm{Pt}}$ reported in various experiments lies between 0.5 to $10 \mathrm{~nm}[11,12]$. In its original form, the ValetFert model did not take spin-flip scattering at interfaces into account [9]. The discontinuity of spin currents at the interfaces was incorporated into the Valet-Fert model in terms of a spin-memory loss (SML) parameter $\delta$ by Baxter et al. in 1999 for nonmagnetic interfaces [16]. An interfacial region of finite thickness $t$ was assumed with a spin-flip diffusion length $l_{\mathrm{I}}$ and the SML was defined to be $\delta=t / l_{\mathrm{I}}$. Since then, $\delta$ has been determined for several nonmagnetic and magnetic|nonmagnetic interfaces at low temperatures, mainly by the Michigan State University group [10, 17]. Almost nothing is known about it at finite temperatures.

Pt has received a lot of attention in the spintronics community in the last decade because of the possibility of it being used to efficiently generate spin currents at room temperature. This effect, known as the "spin-Hall effect" will be discussed in detail in the following section. Because spin currents generated in nonmagnetic metals inevitably undergo bulk and interface spin-flipping, determining the spincurrent generation efficiencies depends on estimating $l_{\mathrm{sf}}$ and $\delta$ correctly.

In this thesis, I undertake a systematic computational study of the spatial behaviour of spin currents as they pass through interfaces and bulk-like materials in multilayers. By using a first-principles computational scheme to calculate conductance and local spin and charge currents for multilayer systems including spin-orbit coupling and thermal and chemical disorder, I will estimate the bulk and interface parameters described above for several material combinations that are important for spin-transport at finite temperatures. 


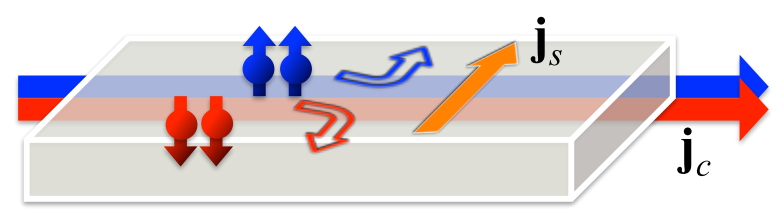

Figure 1.3: Spin Hall effect in a nonmagnetic slab through which a charge current $\mathbf{j}_{c}$ is passed. Spin-up and spin-down electrons aligned perpendicular to $\hat{\mathbf{j}}_{c}$ are scattered in opposite directions. The accumulation of spin-up and spin-down electrons at opposite edges of the samples give rise to a spin current $\mathbf{j}_{s}$. Because there is no charge imbalance between the two edges, this is a pure spin current.

\subsection{Spin Hall effect}

There has been an extensive effort in spintronics to identify phenomena that can be used to generate pure spin currents without using ferromagnetic materials. One such phenomenon is the spin Hall effect (SHE) [18-20] in nonmagnetic heavy transition metals. Nonmagnetic materials have an equal number of arbitrarily oriented spin-up and spin-down electrons in equilibrium. When a charge current is passed through a thin film of nonmagnetic material in the plane of the material, spin-orbit coupling deflects spin-up and spin-down electrons that are oriented perpendicular to the charge current in opposite transverse directions as sketched in Fig. 1.3. The spin accumulation that results at a surface or interface can drive a pure spin current. The relevant parameter that characterizes the spin Hall effect is the spin Hall angle $\Theta_{\mathrm{sH}}$ that is the ratio of the spin current generated per unit charge current.

To estimate $\Theta_{\mathrm{sH}}$, the amount of spin current generated in a nonmagnetic layer is usually detected indirectly by the interaction with an adjacent ferromagnetic layer. Techniques such as nonlocal spin injection [21] and spin-pumping (SP) [22, 23] allowed the SHE to be studied by means of the inverse SHE (ISHE) *. Alternatively, spin currents generated by the SHE could be used to drive the precession of a magnetization by the spin-transfer torque (STT) [24].

To motivate the calculations that will be presented in this thesis, we briefly describe a typical experimental scheme for the SP-ISHE technique in a FM|NM bilayer structure illustrated in Fig. 1.4. Exposure to a microwave frequency electromagnetic field causes the magnetization $\mathbf{M}(t)$ of a ferromagnetic material to precess about a fixed external magnetic field $\mathbf{H}$. The phenomenon of spin-pumping [26, 27] results in the generation of a pure spin current (thick grey arrows in Fig. 1.4) through the interface between the ferromagnetic and nonmagnetic materials. The polarization of the DC component of the spin current is along $\mathbf{H}$. The AC compo-

\footnotetext{
"A spin current polarized perpendicular to the current direction generates a mutually perpendicular charge current
} 
Figure 1.4: Spin pumping and inverse spin Hall effect (ISHE) in a ferromagnetic| nonmagnetic (FM|NM) bilayer. A magnetization $\mathbf{M}(t)$ forced to precess about an external magnetic field $\mathbf{H}$ generates a spin current through the FM|NM interface (thick grey arrows) that flows along the $z$ axis into the NM material. The DC (small, vertical green arrow) and AC (long, horizontal black arrow) components of spin are polarized along the $x$ axis and in the $y-z$ plane, respectively. These DC and AC spin currents generate charge currents by virtue of the ISHE along the $y$ and $x$ axes, respectively that are detected using voltage probes. From Ref. [25], copyright Macmillan Publishers Limited 2014. All rights reserved.

nent, denoted $\sigma(t)$ in Fig. 1.4, is polarized perpendicular to $\mathbf{H}$. Both components of the spin current decay exponentially into the nonmagnetic material away from the interface and generate transverse charge currents by the ISHE [28] that are measured as $\mathrm{DC}$ and $\mathrm{AC}$ voltages, $\mathrm{U}_{\mathrm{DC}}$ and $\mathrm{U}(\mathrm{t})$, respectively. Most experiments only measure the DC part.

Determination of $\Theta_{\mathrm{sH}}$ in a heavy nonmagnetic metal such as Pt using the SPISHE method assumes the knowledge of several underlying parameters. The spin current at the interface, pumped from the ferromagnet (typically permalloy, $\mathrm{Py}=$ $\mathrm{Ni}_{0.8} \mathrm{Fe}_{0.2}$ or Co), is estimated in terms of a spin-mixing conductance $g^{\uparrow \downarrow}$ [27] that depends on the specific FM|NM interface being studied. Spin-orbit coupling at an interface results in an effective spin-mixing conductance parameter $g_{\text {eff }}^{\uparrow \downarrow}$ that contains contributions from the interface spin-memory loss $\delta$ and resistance $R_{\mathrm{I}}$ [29, 30]. As this spin current crosses the interface into the nonmagnetic material, it is attenuated at the interface by interface spin-flipping before undergoing spin-flip diffusion on the length scale $l_{\mathrm{sf}}$ in the bulk of the nonmagnetic layer. To account for the exponentially decaying spin current, experiments measure $U_{D C}$ for multiple samples with different thicknesses of the nonmagnetic layer. Thus, to extract $\Theta_{\mathrm{sH}}$ using SP-ISHE, $g_{\text {eff }}^{\uparrow \downarrow}, \delta, R_{\mathrm{I}}$ and $l_{\text {sf }}$ must either be already known or determined simultaneously.

Because estimates of $l_{\mathrm{sf}}$ for materials like Pt are unreliable [12] and because of a complete lack of any estimates for $\delta$ and $R_{\mathrm{I}}$ for Py|Pt or Co|Pt at finite temperatures, determinations of $\Theta_{\mathrm{sH}}$ have been controversial. Values of $\Theta_{\mathrm{sH}}$ ranging 
from $1.2-39 \%$ have been reported $[11,12]$. The same is true for other techniques such as SHE-STT and nonlocal spin injection. Spin-orbit coupling at an interface is theoretically predicted to enhance the spin Hall effect $[31,32]$ and spin-transfer torque $[33,34]$. However, to avoid introducing a multitude of parameters, most experiments do not account for spin-orbit coupling interface effects. In the last five years, experiments have begun to acknowledge and incorporate interface effects using a variety of advanced models [29, 35-38] but if anything this has led to an increase in the spread of reported values of $\Theta_{\mathrm{sH}}$.

\subsection{Scope of this thesis}

The above discussion provides the motivation for this thesis. We aim to address spin transport in bulk materials such as Pt, Pd, Py, Co and at interfaces between these materials at finite temperatures using first-principles scattering calculations. We pose and address the following questions in the remaining chapters.

- Chapter 2. A scheme to calculate localized spin and charge currents from the results of first-principles scattering calculations is presented. Can the spin-flip diffusion length $l_{\mathrm{sf}}$, the spin-polarization $\beta$ (for ferromagnets) and the spin Hall angle $\Theta_{\mathrm{sH}}$ be calculated for bulk materials free of interface contributions? Are these estimates independent of the choice of computational parameters?

- Chapter 3. How can we extract interface parameters such as the spin-memory loss $\delta$ and interface resistance $R_{\mathrm{I}}$ for interfaces between nonmagnetic metals $(\mathrm{Au} \mid \mathrm{Pt}$ and $\mathrm{Au} \mid \mathrm{Pd})$ from calculations for spin currents and conductance? Is $\delta$ significant for nonmagnetic interfaces? Is there a "giant" interface spin Hall effect for nonmagnetic interfaces analogous to that predicted for Py|Pt interfaces [32]? How do various interface parameters depend on the spin-orbit coupling strength of the heavy metals Pt and Pd? How do these parameters behave as a function of temperature?

- Chapter 4. Generalizing the methodology of Chapter 3, values of $\delta, R_{\mathrm{I}}$ and the interface spin-polarization $\gamma$ are extracted for Py|Pt and Co|Pt interfaces. How do these parameters depend on the magnetic material, magnetic alloy Py vs crystalline Co? How do these parameters behave as a function of temperature? Is there any effect of proximity induced magnetization in Pt on the estimates of these parameters?

- Chapter 5. In recent years several experiments have reported observations of an ISHE in Py [39-42] comparable to that found in Pt. Is a spin Hall effect in Py also found in our calculations using the methods illustrated in Chapter 


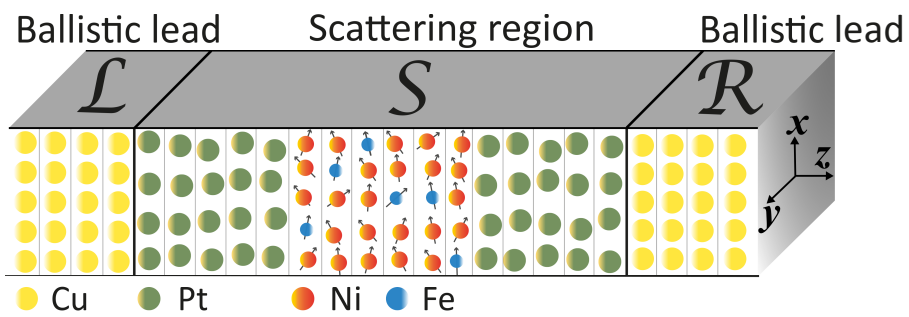

Figure 1.5: Several two dimensional layers of Py and Pt comprise a scattering region $\mathcal{S}$ embedded between semi-infinite crystalline Cu leads on the left $(\mathcal{L})$ and right $(\mathcal{R})$. All atoms in the scattering region are displaced from their equilibrium positions on fcc lattices by thermal disorder. Additionally for Py, the magnetic moments are rotated away from their equilibrium alignment. Because the lattice constants for Pt $\left(a_{\mathrm{Pt}}=3.92 \AA\right)$ and Py $\left(a_{\mathrm{Py}}=3.54 \AA\right)$ are quite different, their in-plane two dimensional unit cells contain different numbers of atoms.

2 and how large is it? If so, what is the microscopic origin of this effect in a light $3 d$ ferromagnetic metal?

\subsection{Computational scheme}

In this section, we give an overview of the method used to perform the calculations that will be presented in this thesis. This method and the corresponding computer codes have been developed over twenty years in the Computational Materials Science group at the University of Twente. At the core of this computational scheme is a two terminal geometry where a scattering structure is embedded between semiinfinite crystalline leads and probed by studying how the Bloch states of the leads are scattered by the disorder of the scattering region. The calculations yield the full scattering matrix and scattering wavefunctions. From these we can determine the response to application of an infinitesimal voltage bias across the leads such as the conductance and spin currents. An example of a scattering structure that will be addressed in Chapter 4 consisting of a Pt|Py|Pt trilayer is shown in Fig. 1.5. By solving the Kohn-Sham equations of density functional theory (DFT) for such a structure, we are able to incorporate realistic electronic structures and models of disorder including thermal lattice and spin disorder in our description of the transport properties.

\subsubsection{Density functional theory}

The solution of the full many-body Schrödinger equation (SE) for $N$ interacting electrons requires finding a wavefunction that is a function of $3 N$ spatial variables. For more than a few electrons this is quite intractable. Hohenberg and Kohn proved 
that the determination of the electronic ground state does not require finding the full many-body wavefunction but only depended on the electron density, a function of only three spatial variables and that the ground state energy functional obeyed a variational principle [43]. Kohn and Sham subsequently made use of the variational principle to derive a single-particle like SE for a system of noninteracting electrons moving in an effective potential that when solved yields the ground state energy of the interacting electron system [44]. This SE must be determined self-consistently with an equation expressing the effective potential in terms of the electron density and another equation expressing the electron density in terms of the single-particle solutions of the SE. These are the so-called Kohn-Sham equations of DFT which was recognized with the award of a Nobel prize (for Chemistry!) in 1998. For magnetic materials, spin-dependent densities must be determined whose sum yields the electron density and whose difference yields the spin density.

\subsubsection{Solving the Kohn-Sham Schrödinger equation for the two ter- minal geometry}

For the $\mathcal{L}|\mathcal{S}| \mathcal{R}$ geometry shown in Fig. 1.5, we solve the Kohn-Sham equation in a matrix representation: $(\mathbf{H}-E \mathbf{O}) \Psi=0[45,46]$. The wavefunction $\Psi$ is expanded in an efficient "tight-binding muffin-tin orbital" (TB-MTO) basis $|i\rangle$ that leads to a vector $\boldsymbol{\Psi}$ of coefficients $\Psi_{i}(i \equiv \mathbf{R} l m \sigma$ where $\mathbf{R}$ is an atom site index and $\operatorname{lm} \sigma$ have their conventional orbital angular momentum and spin meaning). $\mathbf{H}$ and $\mathbf{O}$ are the Hamiltonian and overlap matrices in the localized orbital basis. The Hamiltonian is constructed from Kohn-Sham (KS) potentials for all atoms in the system that have already been calculated self-consistently with a TB-MTO basis along with the ground state charge and spin-densities. The systems we will be interested in include the substitutional alloy Py and interfaces between Pt and Co or Py. A sketch of the TB-MTO basis will be given in Sec. 1.5.3. We first describe the formalism used to evaluate $\boldsymbol{\Psi}$ and how various parameters of interest are extracted from the solutions.

\section{Wave function matching}

The complete wavefunction for the infinite geometry shown in Fig. 1.5 is determined using a two-step "wave function matching" (WFM) scheme [47, 48]. In the first step of this scheme, the Bloch solutions in each semi-infinite lead $(\mathcal{L}$ and $\mathcal{R})$ are determined making use of their translational symmetry to yield eigenmodes that are classified as either left- or right-going solutions. So-called generalized Bloch matrices are used to eliminate the semi-infinite leads, replacing them with energy dependent embedding potentials for all atoms on the boundary layers. This reduces the infinite problem to one of finite size (scattering region plus embedding layers). In the second step, the lead eigenmodes that propagate into the scattering region $\mathcal{S}$ are used as boundary conditions to solve the KS equations for $\mathcal{S}$ by solving a set 
of linear equations numerically. This yields the full scattering matrix consisting of reflection and transmission probability amplitudes as well as the complete wavefunction $\boldsymbol{\Psi}$ throughout the scattering region. From these the interatomic charge and spin currents can be constructed.

\section{Conductance and currents}

Once the transmission matrix $\mathbf{t}$ has been evaluated, the conductance $G$ of the system can be expressed as: $G=e^{2} / h \operatorname{Tr}\left\{\mathbf{t t}^{\dagger}\right\}$ according to the Landauer-Büttiker formalism [49]. This will allow us to extract the resistivity for a bulk material as well as the interface resistance for an $\mathrm{A} \mid \mathrm{B}$ interface between two materials $\mathrm{A}$ and $\mathrm{B}$.

The details of how charge and spin currents are evaluated will be described in Chapter 2. The interatomic currents are averaged over each atomic layer perpendicular to the transport direction $z$ yielding a charge current vector $\mathbf{j}_{c}(z)$ and spin current tensor $\mathbf{j}_{s \alpha}(z)$ with $\alpha \equiv x, y, z$. The spatial profile of these currents allow us to extract the bulk and interface spin transport parameters: the spin-flip diffusion length, spin-Hall angle, spin-memory loss, interface spin-Hall angle and bulk and interface spin-polarization.

\subsubsection{Self-consistent atomic potentials: Linearized muffin-tin orbital basis}

We expand all wavefunctions in a basis of tight-binding muffin-tin orbitals (TBMTO) in the so called atomic spheres approximation (ASA) [50-52]. Since the Kohn-Sham equations are solved self consistently, we assume we have a potential and want to solve the next iteration of the KS cycle. To use separation of variables, we construct an auxiliary potential with so-called muffin-tin form. It is spherically symmetric in non-overlapping spheres centered on the atoms - so-called muffin-tin (MT) spheres - and constant in the interstitial region between these spheres where the KS equation just becomes the wave equation. Spherical symmetry means that the KS equation is separable inside MT spheres and can be solved (numerically) at any given energy $\varepsilon$ in terms of products of $l$ dependent partial waves $\phi_{l}(\varepsilon, r)$ that are functions of the radial variable $r$ and spherical harmonics $Y_{l m}(\theta, \phi)$ that are functions of the angle variables $\theta$ and $\phi$. In the interstitial region the KS equation is just the wave equation that is solved in terms of products of spherical Bessel and Neumann functions and spherical harmonics. As solutions of the wave equation, spherical Bessel and Neumann functions centered on any site can be expanded in terms of spherical Bessel and Neumann functions on any other site where the expansion coefficients are called structure constants. Continuous and differentiable functions are constructed by matching the partial waves inside a MT sphere to appropriate linear combinations of spherical Bessel and Neumann functions. These functions are, however, not normalizable. By substracting the Bessel function from the solu- 
tions in the MT sphere and in the interstitial region, functions are constructed that are continuous, differentiable and normalizable - but no longer solutions of the KS equation inside a MT sphere because of the subtracted Bessel function. The resulting "muffin-tin orbital" can be used to calculate matrix elements of the Hamiltonian eventually including nonspherical components of the full potential. This results in an energy dependent secular equation that can be linearized by (i) expanding the energy dependent partial wave in a Taylor expansion in energy keeping just the partial wave and its first energy derivative evaluated at some fixed energy $\varepsilon_{v}$ so $\phi_{l}(\varepsilon, r) \sim \phi_{l}\left(\varepsilon_{v}, r\right)+\left(\varepsilon-\varepsilon_{v}\right) \dot{\phi}_{l}\left(\varepsilon_{v}, r\right)$ where $\dot{\phi}$ indicates an energy derivative, (ii) expanding the MT spheres so as to completely fill space and (iii) assuming that the kinetic region in the (eliminated!) interstitial region is zero. The expanded MT spheres are called atomic or Wigner-Seitz spheres. This linearized atomic spheres approximation (ASA) has been shown to work very well for close-packed transition metals [50-52].

The basis described above is called a "linearized muffin-tin orbital" (LMTO). When the kinetic energy in the interstitial region is assumed to be zero, the structure constants become "canonical"; i.e. they only depend on the crystal structure. In practice $s, p, d$ and occasionally $f$ orbitals are sufficient. These orbitals have one drawback: they have very long range in real space. This problem can be resolved using a tight-binding (TB) representation of LMTOs. By introducing "screened" structure constants, the long range can be reduced to second or third nearest neighbours. Together, these approximations result in a highly efficient TB-LMTO-ASA scheme that describes the electronic structure of most transition metals very accurately.

\section{Periodic and nonperiodic systems}

Translational periodicity greatly simplifies solution of the electronic structure problem described above. The potential $V(\mathbf{r})$ has the periodicity of the lattice translation vectors $\mathbf{T}$ so $V(\mathbf{T}+\mathbf{r})=V \mathbf{r})$. The consequence of this periodicity is that it is sufficient to determine the wavefunction $\psi(\mathbf{r})$ within a primitive unit cell in order to describe the wavefunction everywhere in the lattice. This follows from Bloch's theorem which states that the solution of the Schrödinger equation for a periodic solid can be labelled by a wavevector $\mathbf{k}$ and has the form $\psi_{\mathbf{k}}(\mathbf{r})=e^{i \mathbf{k} . \mathbf{r}} u_{\mathbf{k}}(\mathbf{r})$ such that $u_{\mathbf{k}}(\mathbf{T}+\mathbf{r})=u_{\mathbf{k}}(\mathbf{r})$. In this thesis, atomic sphere potentials for perfectly periodic solids will be determined using the LMTO-ASA code developed in Stuttgart in the group of O.K. Andersen and referred to simply as "The Stuttgart TB-LMTO-ASA program" ${ }^{\dagger}$.

In the following chapters, we will need to construct scattering regions containing the following nonperiodic configurations of atoms: interfaces, substitutional alloys and combinations of both. In Fig. 1.5, a typical scattering configuration we

\footnotetext{
†https://www2.fkf.mpg.de/andersen/LMTODOC/LMTODOC.html
} 
will encounter is illustrated. Cu (leads) and Pt atomic layers are stacked to form an interface. Py is a substitutional disordered alloy containing two kinds of atoms, $80 \% \mathrm{Ni}$ and $20 \% \mathrm{Fe}$ that randomly occupy fcc lattice sites. In these nonperiodic systems where different atomic species lie in close vicinity to each other, atomic potentials can no longer be calculated independently. Self-consistent potentials for different atomic species along an interface or in an alloy contain contributions from the neighbouring atoms.

To calculate these potentials, we use a code based on the LMTO-ASA basis developed by I. Turek [53] that calculates electronic structures for both periodic and nonperiodic systems using Green's function techniques. In this code, systems consisting of an interface between two atomic species are treated using surface Green's functions. The coherent potential approximation (CPA) [54] is used to describe substitutional alloys. Potentials of different atomic species that make up the alloy are assumed to be present on every lattice site with probabilities corresponding to the stoichiometry of the alloy. The CPA determines an optimal potential iteratively and outputs atomic sphere (AS) potentials for each atomic species.

\subsubsection{Practical implementation}

So far we have discussed specific aspects, ingredients, of the general computational scheme. In practice, it works as sketched in Fig. 1.6. First, some experimental inputs are required to evaluate the AS potentials for a structure such as that shown in Fig. 1.5. These are the composition (nuclear charge) and structure (atomic positions) shown in the dashed boxes on the top left and top center. The information in the top middle box is also required to construct the geometry of the scattering region including interfaces between materials such as Pt and Co or Py with large lattice mismatch. Because the calculations include the effect of temperature-induced disorder, experimental data about temperature dependent resistivities and magnetization (dashed box on top right) for different elements are used to model this thermal disorder. More details of the "Geometry construction" and the modelling of thermal disorder will be discussed in Chapter 2. Once we have the "Electronic structure" of the constitutent atomic species in the form of optimized AS potentials, and the structural arrangement of the atoms in the scattering region, the KohnSham equation is solved to yield the full scattering matrix consisting of matrices of reflection and transmission probability amplitudes as well as all the scattering wavefunctions throughout the scattering region as described in references $[45,46]$ (bold box in the center). From these outputs, we can calculate the conductance and charge and spin currents. This allows us to spin-transport parameters using methods that will be described in the remaining chapters of this thesis (bold dotted box at the bottom). 


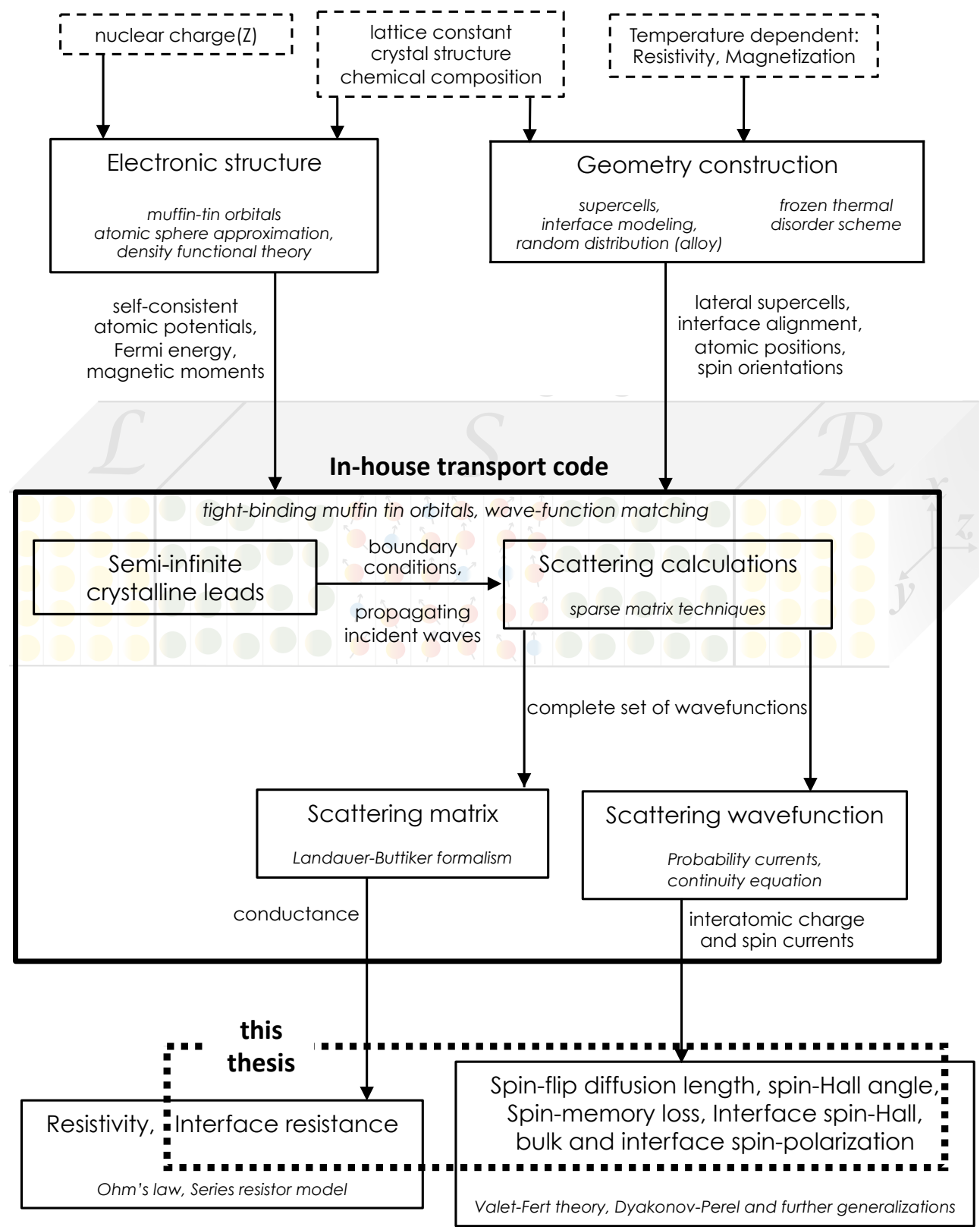

Figure 1.6: The steps required to extract transport parameters from firstprinciple calculations. The italic text indicates the underlying methods/approximations/formalism; the arrows indicate the flow of information. The bold box indicates the computation carried out with our in-house code. The bold dotted box indicates the focus of this thesis. 


\section{2}

\section{Calculating spin transport properties from first principles: spin currents" ${ }^{*}$}

Local charge and spin currents are evaluated from the solutions of fully relativistic quantum mechanical scattering calculations for systems that include temperatureinduced lattice and spin disorder as well as intrinsic alloy disorder. This makes it possible to determine material-specific spin transport parameters at finite temperatures. Illustrations are given for a number of important materials and parameters at $300 \mathrm{~K}$. The spin-flip diffusion length $l_{\mathrm{sf}}$ of Pt is determined from the exponential decay of a spin current injected into a long length of thermally disordered Pt; we find $l_{\mathrm{sf}}^{\mathrm{Pt}}=5.3 \pm 0.4 \mathrm{~nm}$. For the ferromagnetic substitutional disordered alloy Permalloy (Py), we inject currents that are fully polarized parallel and antiparallel to the magnetization and calculate $l_{\mathrm{sf}}$ from the exponential decay of their difference; we find $l_{\mathrm{sf}}^{\mathrm{Py}}=2.8 \pm 0.1 \mathrm{~nm}$. The transport polarization $\beta$ is found from the asymptotic polarization of a charge current in a long length of Py to be $\beta=0.75 \pm 0.01$. The spin Hall angle $\Theta_{\mathrm{sH}}$ is determined from the transverse spin current induced by the passage of a longitudinal charge current in thermally disordered Pt; our best estimate is $\Theta_{\mathrm{sH}}^{\mathrm{Pt}}=4.5 \pm 1 \%$ corresponding to the experimental room temperature bulk resistivity $\rho=10.8 \mu \Omega \mathrm{cm}$.

"Published as: R.J.H. Wesselink, K. Gupta, Z. Yuan, P.J. Kelly, Calculating spin transport properties from first principles: spin currents, Physical Review B 99, 144409 (2019)

${ }^{\dagger}$ R.J.H. Wesselink and K. Gupta contributed equally to this work. The presented methodology was developed and implemented in a computer code by R.J.H. Wesselink. The calculations and analysis in this chapter are carried out by K. Gupta. 


\subsection{Introduction}

Experiments in the field of spintronics are almost universally interpreted using semiclassical transport theories [55]. In such phenomenological theories based upon the Boltzmann or diffusion equations, a number of parameters are used to describe how transport depends on material composition, structure and temperature. For a bulk nonmagnetic material (NM) these are the resistivity $\rho$, the spin flip diffusion length (SDL) $l_{\mathrm{sf}}$ [8-10] and the spin Hall angle (SHA) $\Theta_{\mathrm{sH}}$ that measures the efficiency of the spin Hall effect (SHE) [18-20] whereby a longitudinal charge current is converted to a transverse spin current, or of its inverse [11, 12]. The transport properties of a ferromagnetic material (FM) are characterized in terms of the spin-dependent resistivities $\rho_{\downarrow}$ and $\rho_{\uparrow}$, a SDL $l_{\mathrm{sf}}$ and an anomalous Hall angle (AHA). Instead of $\rho_{\downarrow}$ and $\rho_{\uparrow}$, the polarization $\beta=\left(\rho_{\downarrow}-\rho_{\uparrow}\right) /\left(\rho_{\downarrow}+\rho_{\uparrow}\right)$ and a resistivity $\rho^{*}=\left(\rho_{\uparrow}+\rho_{\downarrow}\right) / 4$ are frequently used. Phenomenological theories ultimately aim to relate currents of charge $\mathbf{j}_{c}$ and spin $\mathbf{j}_{s \alpha}$ to, respectively, gradients of the chemical potential $\mu_{c}$ and spin accumulation $\mu_{s \alpha}$ (where $\alpha$ labels the spin component) in terms of the above parameters but they tell us nothing about the values of the parameters for particular materials or combinations of materials. In this chapter we evaluate these parameters using realistic electronic structures and models of disorder within the framework of density functional theory (DFT).

Ten years ago only a handful of measurements had been made of $l_{\mathrm{sf}}[10,12]$ and $\Theta_{\mathrm{sH}}[11,12]$ in Pt. The polarization $\beta$ had earlier been found to depend on the type of measurement used to extract it. This usually involved an interface [56] and it was only the introduction of current-induced spin-wave Doppler shift measurements [57] that made it possible to probe the current polarization in the bulk of a magnetic material far from any interfaces. The influence of interfaces still plagues measurements of $\Theta_{\mathrm{sH}}$ and $l_{\mathrm{sf}}$ however. The advent of nonlocal spin injection and spin-pumping (SP) allowed the SHA and SDL to be studied by means of the inverse SHE (ISHE). Alternatively, spin currents generated by the SHE could be used to drive the precession of a magnetization by the spin-transfer torque (STT). These innovations have yielded a host of new, mainly room temperature (RT) results $[11,12,58]$. All of these methods involve NM|FM interfaces that introduce a variety of interface-related factors such as spin memory loss and interface spin Hall effects that are not taken into account systematically in the interpretation of the experimental results leading to a large spread in estimates of the SDL and SHA [29]. Perhaps as a result of this, there are few systematic studies of the temperature dependence of $l_{\mathrm{sf}}, \Theta_{\mathrm{sH}}$ and $\beta[59,60]$.

We will demonstrate below how interface effects also present problems for firstprinciples calculations of bulk parameters using scattering theory. This motivated us to develop a method to extract local spin currents from the results of scattering calculations. We will show how this helps to resolve the problems just mentioned and how it will allow us to calculate the parameters $l_{\mathrm{sf}}, \Theta_{\mathrm{sH}}$ and $\beta$ appropriate to 
various bulk materials in a reliable manner. "Bulk" here implies a monocrystalline material with only "intrinsic" alloy disorder or the thermal vibrational or spin disorder that is inherent in experiments at finite temperatures. The disorder resulting from impurities, grain boundaries, stacking faults, surfaces, interfaces etc. that can in principle be avoided in experiment will not be considered here. Such disorder can vary considerably between different experiments and is sometimes used as an effective way of tuning material properties. For example, the SDL and SHA of a metal are found to be significantly different depending on the type of impurities present albeit in the low-concentration and low temperature limits [61, 62]. Interfaces can also strongly enhance the efficiency of spin-charge conversion [32] but may be difficult to distinguish from bulk effects in experiment. In the following we provide a detailed background to the problems encountered in the scattering formalism and how they will be resolved by introducing local currents.

\section{Problems presented by scattering theory}

To simultaneously describe the magnetic and transport properties of transition metals quantitatively requires taking into account their degenerate electronic structures and complex Fermi surfaces. Realistic electronic structures have only been incorporated into Boltzmann transport theory for the particular cases of point impurities [63] and for thermally disordered elemental metals [64]. For the layered structures that form the backbone of spintronics, the most promising way to combine complex electronic structures with transport theory is to use scattering theory formulated either in terms of nonequilibrium Green's functions or wavefunction matching [55] that are equivalent in the linear response regime [65]. The effect of temperature on transport has been successfully included in scattering calculations in the adiabatic approximation by constructing scattering regions with temperature-induced lattice and spin disorder $[66,67]$. By constructing charge and spin currents (and chemical potentials [68]) from the scattering theory solutions, we aim to make contact with the phenomenological theories that are formulated in terms of these quantities. Though we will be focusing on bulk transport properties in this chapter, the methodology we present will be directly extended to interfaces in Chapter 3 and Chapter 4.

Indeed, in a two-terminal $\mathcal{L}|\mathcal{S}| \mathcal{R}$ scattering formalism where a "scattering" region $\mathcal{S}$ is probed by attaching left $(\mathcal{L})$ and right $(\mathcal{R})$ leads to study how incoming Bloch states in the leads are scattered into outgoing states, interfaces are unavoidable and must be factored into (or out of) any subsequent analysis. For example, an interface gives rise to an interface resistance even in the absence of disorder because of the electronic structure mismatch between different materials [45, 6971]. For disordered materials, the linear dependence of the resistance $R$ on the length $L$ of the scattering region allows the interface contribution to be factored out by extracting the bulk resistivity from the linear part of $R(L)[46,72]$. An anal- 


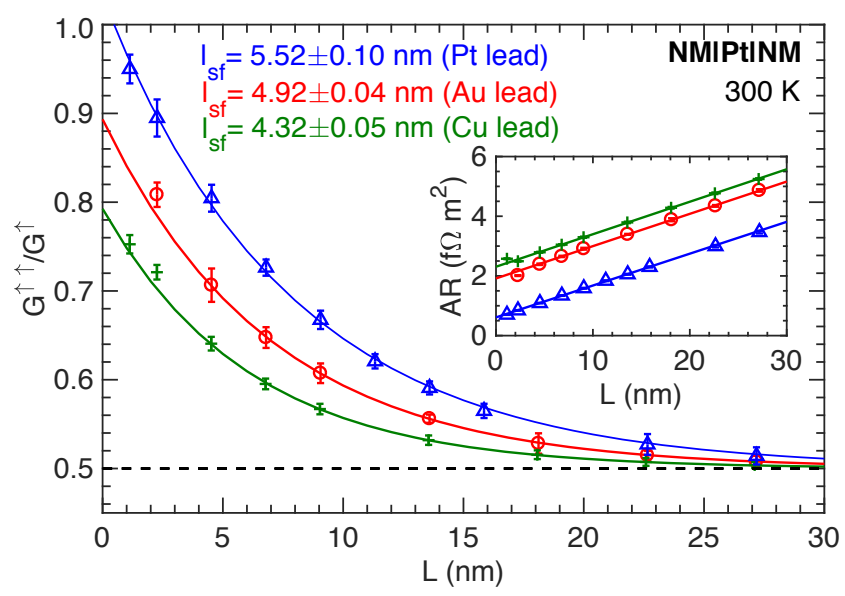

Figure 2.1: Calculated fractional spin conductance $G^{\uparrow} / G^{\uparrow}$ for RT thermally disordered Pt sandwiched between the different ballistic leads: Pt (blue triangles), Au (red circles) and $\mathrm{Cu}$ (green crosses). $G^{\sigma \sigma^{\prime}}$ is ( $e^{2} / h$ times) the transmission probability of a spin $\sigma$ from the left hand lead into a spin $\sigma^{\prime}$ in the right hand lead; $G^{\uparrow}=G^{\uparrow \uparrow}+G^{\uparrow \downarrow}$. The solid lines are the exponential fits to the calculated values giving rise to $l_{\mathrm{sf}}$. Inset: The areal resistance of the NM|Pt|NM as a function of the length $L$ of Pt for all three ballistic leads. Solid lines are linear fits whose slopes yield identical resistivities in the three cases. Data for $L<4 \mathrm{~nm}$ is excluded from the linear fit [46].

ogous procedure can be applied to study the magnetization damping [30, 46, 72] where interfaces give rise to important observable effects [55].

In the case of spin-flipping, the exponential dependence on $L$ of the transmission probability $T^{\sigma \sigma^{\prime}}$ of states with spin $\sigma$ from one lead into states with spin $\sigma^{\prime}$ in the other lead makes this numerically challenging. Starikov, Liu and coworkers used $T^{\sigma \sigma^{\prime}}$ to evaluate the SDL in $\mathrm{Fe}_{x} \mathrm{Ni}_{1-x}$ disordered alloys [72] and in thermally disordered Pd and Pt [30]. In terms of the corresponding spin-resolved conductances $G^{\sigma \sigma^{\prime}}=\frac{e^{2}}{h} T^{\sigma \sigma^{\prime}}$, the total conductance of spin $\sigma$ is given by $G^{\sigma}=$ $\sum_{\sigma^{\prime}} G^{\sigma \sigma^{\prime}}$ and the total conductance of the system is the sum over both possible spins: $G=\sum_{\sigma \sigma^{\prime}} G^{\sigma \sigma^{\prime}}$. For a single spin channel, Liu et al. identified the exponential decay of the "fractional spin conductance" $G^{\uparrow \uparrow} / G^{\uparrow}$ with the "spin diffusion length" $l_{\uparrow}$. In Fig. 2.1 we show $G^{\uparrow \uparrow} / G^{\uparrow}$ for RT thermally disordered Pt and different lead materials. The lattice disorder in the scattering region is taken to be Gaussian with a mean-square displacment chosen to reproduce the experimental room temperature resistivity $\rho=10.8 \mu \Omega \mathrm{cm}$ [73]. Using ballistic Pt leads, we calculate the (blue) curve indicated with open triangles in Fig. 2.1 from which we obtain a value of $l_{\uparrow}=7.8 \pm 0.3 \mathrm{~nm}$. Because Pt is spin degenerate, $l_{\downarrow} \equiv l_{\uparrow}$ and [9] 
$l_{\mathrm{sf}}=\left(l_{\uparrow}^{-2}+l_{\downarrow}^{-2}\right)^{-1 / 2}=5.52 \pm 0.10 \mathrm{~nm}$ in agreement with Ref.[30]. For $L \sim 1 \mathrm{~nm}$, we see that $G^{\uparrow \uparrow} \sim G^{\uparrow}$ indicative of a very weak interface between ballistic Pt leads and thermally disordered Pt. When we use Au leads however, the effect of the interface becomes more noticeable and the value of $l_{\mathrm{sf}}$ is reduced to $\sim 4.9 \mathrm{~nm}$. Because of the large difference of $\sim 8.5 \%$ between the lattice constants of $\mathrm{Pt}$ and $\mathrm{Cu}$, to study an interface between them we use an $8 \times 8$ lateral supercell of $\mathrm{Cu}$ to match to a $2 \sqrt{13} \times 2 \sqrt{13}$ lateral supercell of Pt. In this case, the interface is even stronger and we find an even shorter value of $l_{\mathrm{sf}} \sim 4.3 \mathrm{~nm}$. This dependence of $l_{\mathrm{sf}}$ on the lead material is unsatisfactory.

For an ohmic material, the conductance decays as $1 / L$ and it is relatively easy to separate out interface effects by plotting the resistance $R=1 / G$ as a function of $L$ to determine the resistivity $\rho$, eventually ignoring short values of $L$ not characteristic of the bulk material as illustrated in the inset to Fig. 2.1. However, in the SDL case where the partial conductances decay exponentially, it is numerically much less straightforward to eliminate interface contributions. Ignoring too many small values of $L$ leaves us with too few data points with which to determine $l_{\text {sf }}$ accurately. Unfortunately, we do not know a priori how far the effect of the interface extends. Similar considerations apply to the determination of $l_{\mathrm{sf}}$ for a ferromagnetic material when we examine the effect of using different lead materials.

\section{How these problems can be solved}

Local spin currents provide a description of the scattering region layer by layer. Contributions from interfaces show up only in layers close to the interfaces and not deep in the bulk. We will resolve the problems discussed above by evaluating spin currents as a function of $z$ from the results of scattering calculations that include temperature-induced lattice and spin disorder as well as alloy disorder but do not assume diffusive behaviour a priori. By focussing on the currents and chemical potentials employed in semiclassical theories [8] such as the Valet-Fert (VF) formalism [9] that are widely used to interpret experiments, we will be able to evaluate the parameters that occur in those formalisms. For example, we will be able to determine the SDL $l_{\mathrm{sf}}$ from the exponential decay of a spin current injected into a long length of thermally disordered material. The transport polarization $\beta$ of the ferromagnetic substitutional disordered alloy Permalloy ( $\mathrm{Py}, \mathrm{Fe}_{20} \mathrm{Ni}_{80}$ ) will be determined straighforwardly from the asymptotic polarization of a charge current. The spin Hall angle $\Theta_{\mathrm{sH}}$ of Pt will be found from the transverse spin current induced by the passage of a longitudinal charge current. We will demonstrate that we can treat sufficiently long scattering regions as to be able to distinguish bulk and interface behaviour in practice. In Chapter 3 and Chapter 4, we will study the interface contributions explicitly in order to extract interface parameters for various $\mathrm{NM} \mid \mathrm{NM}^{\prime}$ and $\mathrm{FM} \mid \mathrm{NM}$ interfaces.

The plan of this chapter is as follows. We begin Sec. 2.2 with a summary of 
the phenomenological Valet-Fert formalism (Sec. 2.2.1) containing the parameters we aim to evaluate. Sec. 2.2.2 outlines the quantum mechanical formalism that results in scattering wavefunctions which we will use to calculate position resolved charge and spin currents. In Sec. 2.2.3 we explain how currents between pairs of atoms are calculated using the scattering wavefunctions. Sec. 2.2.4 explains how layer averaged currents are constructed from the interatomic currents. The most important practical aspects of scattering calculations that determine the accuracy of the computational results are reviewed in Sec. 2.2.5. In Sec. 2.3 we illustrate the foregoing methodology by calculating $l_{\mathrm{sf}}$ for Pt (2.3.1), $l_{\mathrm{sf}}$ (2.3.3) and $\beta$ (2.3.2) for Py, and $\Theta_{\mathrm{sH}}$ for Pt (2.3.4). The emphasis in this chapter will be on studying how the parameters depend on computational details of the scattering calculations such as lateral supercell size, Brillouin zone (BZ) sampling, basis set etc. A comparison with experiment and other calculations is made in Section 2.4. Our results are summarized and some conclusions are drawn in Section 2.5.

\subsection{Methods}

\subsubsection{Semiclassical transport theory}

In this section, we recapitulate the VF description of spin transport that characterizes transport in axially symmetric "current perpendicular to the plane" (CPP) geometries in terms of the material-specific parameters $\rho_{\sigma}$ and $l_{\mathrm{sf}}$. Starting from the Boltzmann formalism, Valet and Fert [9] derived the following macroscopic equations for a current flowing along the $z$ direction perpendicular to the interface plane in response to gradients of the chemical potential

$$
\begin{aligned}
& \frac{\partial^{2} \mu_{s}}{\partial z^{2}}=\frac{\mu_{s}}{l_{\mathrm{sf}}^{2}}, \\
& j_{\sigma}(z)=-\frac{1}{e \rho_{\sigma}} \frac{\partial \mu_{\sigma}}{\partial z} .
\end{aligned}
$$

With respect to a quantization axis taken to be the $z$ axis, the majority and minority spin-polarized current densities and chemical potentials are denoted by $j_{\sigma}$ and $\mu_{\sigma}$ respectively with $\sigma=\uparrow$ (majority) or $\downarrow$ (minority). $\mu_{s} \equiv \mu_{s z}=\mu_{\uparrow}-\mu_{\downarrow}$ and $\rho_{\sigma}$ is the spin-dependent bulk resistivity. According to the two-current series resistor model [9], resistances are first calculated separately for spin up and spin down electrons and then added in parallel. For non-magnetic materials, $\rho_{\uparrow}=\rho_{\downarrow}=2 \rho$, where $\rho$ is the total resistivity. Thus, spin transport in the bulk of a material can be characterized in terms of its resistivity $\rho$ and SDL $l_{\mathrm{sf}}$. Equations (2.1a) and (2.1b) can be solved for $\mu_{\uparrow}, \mu_{\downarrow}, j_{\uparrow}$, and $j_{\downarrow}$ making use of the condition that the total current density $j=j_{\uparrow}+j_{\downarrow}$ is conserved in one-dimensional transport. Dropping the "sf" subscript when there is no danger of confusion, the general solution of (2.1a) is 
$\mu_{s}(z)=A e^{z / l}+B e^{-z / l}$. The normalized effective spin-current density $\widehat{j_{s}} \equiv j_{s z}^{z} / j=$ $\left[j_{\uparrow}(z)-j_{\downarrow}(z)\right] / j$ is given by

$$
\widehat{j_{s}}(z)=\beta-\frac{1}{2 e j \rho^{*} l}\left[A e^{z / l}-B e^{-z / l}\right]
$$

where the coefficients $A$ and $B$ can be determined by using appropriate boundary conditions. For a NM material $\beta=0$. We will be concerned with calculating $\widehat{j_{s}}(z)$ from the results of two-terminal scattering calculations for $\mathcal{L}|\mathcal{S}| \mathcal{R}$ configurations. The coefficients $A_{\mathcal{L}}, B_{\mathcal{L}}, A_{\mathcal{S}}, B_{\mathcal{S}}, A_{\mathcal{R}}$ and $B_{\mathcal{R}}$ will be determined by imposing suitable boundary conditions at the $\mathcal{L} \mid \mathcal{S}$ and $\mathcal{S} \mid \mathcal{R}$ interfaces.

\section{Spin-flip diffusion length}

Equation (2.2) provides a simple prescription for extracting the SDL from a calculation of the spin current ${\widehat{j_{s}}}_{(z)}$. In the case of a non-magnetic material, we choose the left lead to be ferromagnetic, e.g. a half metallic ferromagnet, so the current entering the non-magnetic material is fully polarized with $\left(\left|\widehat{j_{s}}(0)\right|=1\right)$. The right lead is nonmagnetic so $\widehat{j_{s}}(z) \rightarrow 0$ in the limit of large $z$. The boundary condition for the right lead in this limit is $\widehat{j_{s}}(\infty)=0$ so $\widehat{j_{s}}(z)=C \exp (-z / l)$ and $l_{\text {sf }}$ can be determined from the slope of $\ln {\widehat{j_{s}}}_{s}(z)$.

\section{Polarization}

For a symmetric NM|FM|NM configuration with a thickness $L$ of FM, we choose the origin at the middle of the FM layer so $B=-A$ in (2.2) and the spin current has the form

$$
\widehat{j_{s}}(z)=\frac{j_{\uparrow}(z)-j_{\downarrow}(z)}{j}=\beta-c \cosh \frac{z}{l}
$$

and $\widehat{j_{s}}(z) \rightarrow \beta$ for scattering regions much longer than $l_{\mathrm{sf}}$.

\section{Spin-Hall angle}

The spin Hall effect is such that passage of a charge current through a diffusive nonmagnetic material leads to the generation of transverse spin currents $j_{s \alpha}^{\perp}$ where $\alpha$ labels the direction of spin polarization that is given by the vector product of the driving charge current (assumed to be in the $z$ direction) and the induced transverse spin current $(\perp)$. For a constant charge current density $j$, the normalized transverse spin current sufficiently far from the interfaces gives the spin Hall angle $\Theta_{\mathrm{sH}}=\widehat{j}_{s}^{\perp} \equiv$ $j_{s \alpha}^{\perp} / j$. 


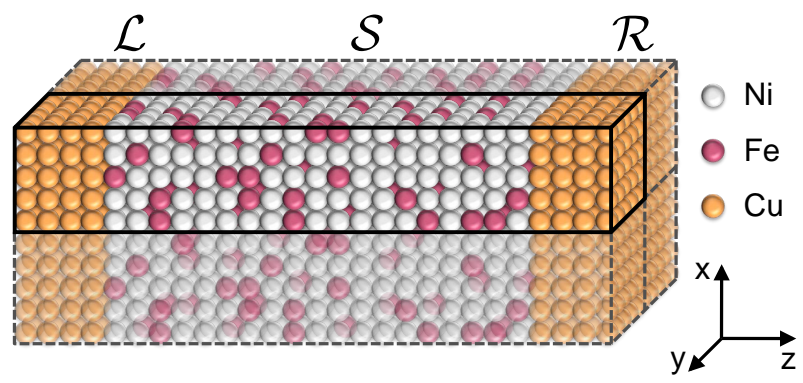

Figure 2.2: Example of a transverse supercell for a $\mathrm{Cu}|\mathrm{Py}| \mathrm{Cu}$ scattering geometry. $\mathrm{Cu}$ atomic layers form semi-infinite ballistic leads denoted $\mathcal{L}$ and $\mathcal{R}$. The scattering region $\mathcal{S}$ consists of a thickness $L$ of the substitutional disordered $\mathrm{Ni}_{80} \mathrm{Fe}_{20}$ alloy, Permalloy, sandwiched between the leads. Each atomic layer in $\mathcal{L}|\mathcal{S}| \mathcal{R}$ contains $5 \times 5$ atoms. The layers are parallel to the $x y$ plane and in the calculations this structure is repeated in the $x$ and $y$ directions so that an infinite periodic structure arises.

\subsubsection{Quantum Mechanical Scattering}

The starting point for our determination of $\mathbf{j}_{c}$ and $\mathbf{j}_{s \alpha}$ is the solution of a singleparticle Schrödinger equation $* H \Psi=E \Psi$ for a two terminal $\mathcal{L}|\mathcal{S}| \mathcal{R}$ configuration in which a disordered scattering region $\mathcal{S}$ is sandwiched between crystalline left$(\mathcal{L})$ and right-hand $(\mathcal{R})$ leads, Fig. 2.2. The quantum mechanical calculations are based upon Ando's wave-function matching (WFM) $[47,48]$ method formulated in terms of a localized orbital basis $|i\rangle$. Our implementation $[45,46]$ is based upon a particularly efficient minimal basis of tight-binding muffin tin orbitals (TB-MTOs) [51, 74, 75] with $i=R \operatorname{lm} \sigma$ in combination with the atomic spheres approximation (ASA) [50]. Here $R$ is an atom site index and $l m \sigma$ have their conventional meaning. In terms of the basis $|i\rangle$, the wavefunction $\Psi$ is expressed as

$$
|\Psi\rangle=\sum_{i}|i\rangle\langle i \mid \Psi\rangle
$$

and the Schrödinger equation becomes a matrix equation with matrix elements $\langle i|H| j\rangle . \Psi$ is a vector of coefficients with elements $\psi_{i} \equiv\langle i \mid \Psi\rangle$ extending over all sites $R$ and over the orbitals on those sites, for convenience collectively labelled as $i_{R}$.

A number of approximations makes solution of the infinitely large system tractable. First, by making use of their translational periodicity, the WFM method eliminates the semiinfinite leads by introducing an energy dependent "embedding potential"

\footnotetext{
${ }^{*}$ In the framework of density functional theory $[43,44]$ these are the Kohn-Sham equations.
} 
on each atom in the layer of atoms bounding the scattering region $[47,48]$. Second, the system is assumed to be periodic in the directions transverse to the transport direction (taken to be the $z$-axis). This makes it possible to characterize the scattering states with a transverse Bloch wavevector $\mathbf{k}_{\|}$. Fixing $\mathbf{k}_{\|}$and the energy (typically, but not necessarily, at $E=E_{F}$ ), the Schrödinger equation is first solved for each lead yielding several eigenmodes $\mu$ and their corresponding wavevectors $k_{\perp \mu}$. For propagating solutions $k_{\perp}$ must be real. By calculating the velocity vectors $\mathbf{v}$ for $\mathbf{k}_{\mu} \equiv\left(\mathbf{k}_{\|}, \mathbf{k}_{\perp \mu}\right)$, propagating modes in both leads can be classified as right-going " $\mathbf{v}^{+}$" or left-going " $\mathbf{v}^{-}$". To simplify the notation, we rewrite $\mathbf{k}_{\mu} \equiv \mu \mathbf{k}$. The lead solutions are then used as boundary conditions to solve the Schrödinger equation in the scattering region for states transmitting from left to right $(L \rightarrow R)$ and right to left $(R \rightarrow L)$. The complete wavefunction can be written as

$$
\Psi_{\mu \mathbf{k}}^{+}=\left(\begin{array}{c}
\Psi_{\mu \mathbf{k}}^{\mathcal{L}+}+\sum_{\nu \mathbf{l}} r_{\nu \mathbf{l}, \mu \mathbf{k}} \Psi_{\nu \mathbf{l}}^{\mathcal{L}-} \\
\Psi_{\mu \mathbf{k}}^{\mathcal{S}+} \\
\sum_{\nu \mathbf{l}} t_{\nu \mathbf{l}, \mu \mathbf{k}} \Psi_{\nu \mathbf{l}}^{\mathcal{R}+}
\end{array}\right)
$$

and

$$
\Psi_{\mu \mathbf{k}}^{-}=\left(\begin{array}{c}
\sum_{\nu \mathbf{l}} t_{\nu \mathbf{l}, \mu \mathbf{k}} \Psi_{\nu \mathbf{l}}^{\mathcal{L}-} \\
\Psi_{\mu \mathbf{k}}^{\mathcal{S}-} \\
\Psi_{\mu \mathbf{k}}^{\mathcal{R}-}+\sum_{\nu \mathbf{l}} r_{\nu \mathbf{l}, \mu \mathbf{k}} \Psi_{\nu \mathbf{l}}^{\mathcal{R}+}
\end{array}\right)
$$

where $t$ and $r$ are matrices of transmission and reflection probability amplitudes. Because the leads contain no disorder by construction, we will be focusing on the wave functions $\Psi_{\mu \mathbf{k}}^{\mathcal{S}_{ \pm}}$of (2.5) and (2.6) in the scattering region to calculate the current tensor separately for $\Psi^{\mathcal{S}^{+}}(L \rightarrow R)$ and $\Psi^{\mathcal{S}^{-}}(R \rightarrow L)$ summed over all $\mu \mathbf{k}$. The former yields a right going electron current whereas the latter yields a left going hole current when an infinitesimal voltage bias is applied.

\subsubsection{Calculating the full current tensor}

In this section we discuss a method to calculate from first principles charge and spin currents between atoms using localized orbitals. This is particularly suited for methods using the ASA and TB-MTOs [51, 74, 75]. In an independent electron picture (in the framework of DFT), the particle density is given by $n(\mathbf{r}, t)=|\Psi(\mathbf{r}, t)|^{2}$ where we omit the subscripts $\mu \mathbf{k}$ and superscripts \pm of the previous section. Particle conservation requires that $\partial_{t} n(\mathbf{r}, t)+\nabla \cdot \mathbf{j}(\mathbf{r})=0$ where $\mathbf{j}(\mathbf{r})$ is the probability current density. A volume integral over the atomic sphere (AS) $S_{P}$ centered on atom $P$ 
yields

$$
\partial_{t} n_{P}=-\iint_{S_{P}} \mathbf{j} \cdot d \mathbf{S}
$$

where $n_{P}$ is the number of particles in the AS that can only change in time if a current flows in or out of the atomic sphere. The ASA requires filling all of space with atomic Wigner Seitz spheres and leads to a discretized picture in which the net current into or out of $S_{P}$ is balanced by the sum of currents leaving or entering the neighbouring atomic spheres. This interpretation works especially well when we use TB-MTOs whose hopping range is limited to second or third nearest neighbors $[51,74,75]$.

The coefficients in $\Psi$ relating to the basis on atom $P$ can be labelled $\Psi_{P}$

$$
\left|\Psi_{P}\right\rangle=\widehat{P}|\Psi\rangle
$$

with

$$
\widehat{P}=\sum_{i_{P}}\left|i_{P}\right\rangle\left\langle i_{P}\right|
$$

The number of electrons $n_{P}$ on atom $P$ is then defined as

$$
n_{P}=\langle\Psi|\widehat{P}| \Psi\rangle \equiv\left\langle\Psi_{P} \mid \Psi_{P}\right\rangle
$$

where the bra-ket notation implies an inner product. We denote the net current from atom $Q$ to atom $P$ with $j_{c}^{P Q}$, measured in units of the electron charge $-e$ where $e$ is a positive quantity. A sub-block of the Hamiltonian containing the hopping elements from atom $Q$ to atom $P$ is denoted $H_{P Q}$.

Similarly, the $\alpha$ component of the spin density on atom $P$ is

$$
s_{\alpha, P}=\left\langle\Psi_{P}\left|\sigma_{\alpha}\right| \Psi_{P}\right\rangle
$$

where $\sigma_{\alpha}$ is a Pauli matrix. For convenience we divide the spin density by $\hbar / 2$ and express it as a particle density. We also express the spin current as a particle current. $j_{s \alpha}^{P Q}$ is the spin transfer into atomic sphere $P$ carried by electrons hopping from atom $Q$.

The formalism described here is generally applicable with any basis of atomcentered orbitals. The interatomic currents can be simply interpreted in terms of electron hopping between orbitals centered on different atoms; as long as the hopping Hamiltonian is well defined [75-77] there is no further ambiguity. Particle conservation is ensured by (2.7) and numerically verified.

\section{Interatomic electron currents}

With the above definitions, we can rewrite the charge conservation equation (2.7) as

$$
\partial_{t} n_{P}=\sum_{Q} j_{c}^{P Q}\left(\Psi_{P}, \Psi_{Q}\right)
$$


where $j_{c}^{P Q}\left(\Psi_{P}, \Psi_{Q}\right)$ should change sign if $P$ and $Q$ are interchanged; the current from $Q$ to $P$ is minus the current from $P$ to $Q$. The current $j_{c}^{P Q}$ cannot depend on electron densities located elsewhere than on $Q$ or $P$ in an independent electron picture. Note that $j_{c}^{P P}=0$ in accordance with particle conservation. In the Schrödinger picture we have

$$
\partial_{t} \Psi=\frac{1}{i \hbar} H \Psi .
$$

From this we can deduce that with any general time-dependent wavefunction $\Psi$ at a specific moment in time, the number of electrons on atom $P$ changes with the following rate

$$
\begin{aligned}
& \partial_{t} n_{P}=\left\langle\Psi|\hat{P}| \partial_{t} \Psi\right\rangle+\left\langle\partial_{t} \Psi|\hat{P}| \Psi\right\rangle \\
& =\frac{1}{i \hbar} \sum_{Q}\left[\left\langle\Psi_{P}\left|H_{P Q}\right| \Psi_{Q}\right\rangle-\left\langle\Psi_{Q}\left|H_{Q P}\right| \Psi_{P}\right\rangle\right]
\end{aligned}
$$

which has the form of (2.12) with

$$
j_{c}^{P Q}=\frac{1}{i \hbar}\left[\left\langle\Psi_{P}\left|H_{P Q}\right| \Psi_{Q}\right\rangle-\left\langle\Psi_{Q}\left|H_{Q P}\right| \Psi_{P}\right\rangle\right] .
$$

It is easy to see from this expression that solving the time-independent Schrödinger equation $H \Psi=E \Psi$ makes sure the charge on an atom stays constant. This formula can be used to calculate interatomic electron currents.

\section{Interatomic spin currents}

The general form of the time dependence of the spin density on atom $P$ is similar to (2.12)

$$
\partial_{t} s_{\alpha, P}=\sum_{Q} j_{s \alpha}^{P Q}\left(\Psi_{P}, \Psi_{Q}\right)
$$

because spin is carried by electrons. $j_{s \alpha}^{P Q}$ is now not required to change sign if $Q$ and $P$ are interchanged because spin is not conserved ${ }^{\S}$; it changes due to exchange torque as well as spin-orbit torque. This also means that $j_{s \alpha}^{P P}$ need not be zero and in fact it is the local torque on the spin density at $P$. Physically the rate of change of the total spin in a certain region consists of two contributions: the net spin flow into the region and a local torque, i.e.

$$
\partial_{t} s_{\alpha, P}=-\iint_{S_{P}} \mathbf{j}_{s \alpha} \cdot d \mathbf{S}+\tau_{\alpha, P} .
$$

The general form of (2.16) is consistent with the spin conservation equation (2.17).

\footnotetext{
${ }^{\S}$ If spin-orbit coupling is neglected, only the component of spin perpendicular to the magnetization is not conserved.
} 
From the Schrödinger equation we calculate the rate of change of spin on atom $P$ to be

$$
\begin{aligned}
& \partial_{t} s_{\alpha, P}=\left\langle\Psi_{P}\left|\sigma_{\alpha}\right| \partial_{t} \Psi_{P}\right\rangle+\left\langle\partial_{t} \Psi_{P}\left|\sigma_{\alpha}\right| \Psi_{P}\right\rangle \\
& \quad=\frac{1}{i \hbar} \sum_{Q}\left[\left\langle\Psi_{P}\left|\sigma_{\alpha} H_{P Q}\right| \Psi_{Q}\right\rangle-\left\langle\Psi_{Q}\left|H_{Q P} \sigma_{\alpha}\right| \Psi_{P}\right\rangle\right]
\end{aligned}
$$

which has the form of (2.16) with

$$
j_{s \alpha}^{P Q}=\frac{1}{i \hbar}\left[\left\langle\Psi_{P}\left|\sigma_{\alpha} H_{P Q}\right| \Psi_{Q}\right\rangle-\left\langle\Psi_{Q}\left|H_{Q P} \sigma_{\alpha}\right| \Psi_{P}\right\rangle\right] .
$$

If basis functions are defined within the ASA it is very clear that this is the spin current exactly at the sphere boundary of atom $P$ if $Q \neq P$ and it is the local torque if $Q=P$.

As mentioned above, the change of spin in a sphere is the local torque $j_{s \alpha}^{P P}$ plus the sum of all spin currents $j_{s \alpha}^{P Q}$ into the sphere. The spin current leaving sphere $Q$ is not the same as the spin current entering sphere $P$, i.e. $j_{s \alpha}^{P Q} \neq-j_{s \alpha}^{Q P}$ because spin is not conserved. This means there must also be torques acting on spins when they are "between" the atoms in addition to the torques inside the spheres. A torque is of course equal to the rate of change of spin. It can be relevant to compare this way of calculating the local torques to other methods [78].

\subsubsection{Layer averaged current tensor}

The information obtained from the calculations outlined in the previous section has the form of a network flow or a weighted graph. Every node in the graph represents an atom and each end of a connection is accompanied by 4 numbers representing currents. These currents can be arranged in a 4-vector for convenience: $\mathbf{j}^{P Q}=\left(j_{c}^{P Q}, j_{s x}^{P Q}, j_{s y}^{P Q}, j_{s z}^{P Q}\right)$. The problem we now address is how to convert this information to a continuum current density tensor represented on a discrete grid. We start by separating the system into layers $l$. If there is periodicity in the $x$ and $y$ directions (or if the system is finite) this will define cells with volumes $V_{l}$ depending on the thicknesses of the layers. If there is periodicity in the $x y$ plane, we need to characterize equivalent atoms $T$ and $T^{\prime}$ by the unit cell $\mathbf{R}$ they are in, in order to know in which direction an interatomic current is flowing, see Fig. 2.3. That can be done by decomposing the Hamiltonian

$$
H=\sum_{\mathbf{R}} H_{\mathbf{R}} e^{i \mathbf{k} \cdot \mathbf{R}}
$$

and calculating the currents, e.g. $\mathbf{j}^{P T}$ and $\mathbf{j}^{P T^{\prime}}$, for each term separately. We label every atom in the unit cell and every relevant translation of it with a different index ( $P$ or $Q$ here). Note that in $\mathbf{j}^{P Q}$ every atom $P$ lies inside the original unit cell; $Q$ 


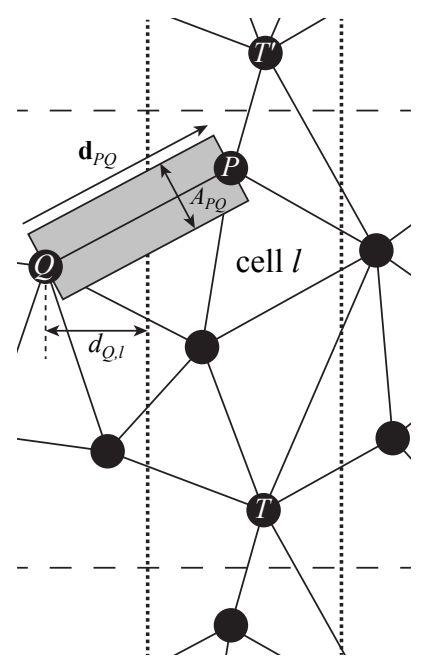

Figure 2.3: Illustration of a number of concepts defined in the text. Filled black circles represent atoms. Current flow is in the $z$ direction from left to right. The horizontal dashed lines indicate the (lateral) unit cell boundaries. The vertical dotted lines indicate layer boundaries in the $z$ direction. The gray area is a "wire" with assumed homogeneous current density that substitutes for the general spatial distribution of the current between $Q$ and $P$, which can therefore be left unknown.

can be either inside or outside. This way we are sure that we count all the currents that should be attributed to one unit cell exactly once. Details of how a current $\mathbf{j}^{P Q}$ is distributed in space are not known so we imagine that the flow is homogeneous in a wire with arbitrary cross-section $A_{P Q}$ and volume $V_{P Q}=A_{P Q}\left|\mathbf{d}_{P Q}\right|$, where $\mathbf{d}_{P Q}$ is the vector pointing from atom $Q$ to $P$.

The current density tensor integrated over the volume of this wire is $\stackrel{\leftrightarrow}{j}^{P Q} V_{P Q}=$ $\mathbf{j}^{P Q} \otimes \mathbf{d}_{P Q}$ and does not depend on the cross-section. The average current density tensor times the volume of cell $l, \stackrel{\leftrightarrow}{j_{l}} V_{l}$, is now the sum of current densities of all these wires integrated within cell $l$. We define a parameter that indicates how much of the wire $P Q$ lies outside the cell at the atom $Q$ end

$$
\beta_{Q P, l}= \begin{cases}0 & \text { if } Q \text { inside cell } l \\ d_{Q, l} / d_{z, Q P} & \text { if } Q \text { outside cell } l\end{cases}
$$

where $d_{P, l}$ is the $z$-distance from atom $P$ to the closest boundary plane of layer $l$. Since the spin current changes between $Q$ and $P$, we make a linear interpolation

$$
\mathbf{j}^{P Q}(c)=c \mathbf{j}^{P Q}-(1-c) \mathbf{j}^{Q P},
$$

where $c$ is a parameter that runs from 0 to 1 depending on the position between 
$Q$ and $P$. Now the part of $\stackrel{\leftrightarrow}{j} P Q V_{P Q}$ that should be counted into $\stackrel{\leftrightarrow}{j_{l}} V_{l}$ is

$$
\begin{aligned}
\int_{\beta_{Q P, l}}^{1-\beta_{P Q, l}} & \mathbf{j}^{P Q}(c) \otimes \mathbf{d}_{P Q} \mathrm{~d} c= \\
\frac{1}{2} & {\left[\left(1-\beta_{P Q, l}\right)^{2}-\left(\beta_{Q P, l}\right)^{2}\right] \mathbf{j}^{P Q} \otimes \mathbf{d}_{P Q} } \\
+\frac{1}{2} & {\left[\left(1-\beta_{Q P, l}\right)^{2}-\left(\beta_{P Q, l}\right)^{2}\right] \mathbf{j}^{Q P} \otimes \mathbf{d}_{Q P} . }
\end{aligned}
$$

Note that $\mathbf{d}_{Q P}=-\mathbf{d}_{P Q}$. The average current density tensor in cell $l$ is then

$$
\stackrel{\leftrightarrow}{j_{l}}=\frac{1}{V_{l}} \sum_{P, Q} \frac{1}{2}\left[\left(1-\beta_{P Q, l}\right)^{2}-\left(\beta_{Q P, l}\right)^{2}\right] \mathbf{j}^{P Q} \otimes \mathbf{d}_{P Q}
$$

Now we can multiply with the cross-sectional area of the unit cell to obtain a total current per unit voltage between two leads that can be compared directly with the total Landauer-Büttiker conductance. This is an important criterion to verify the numerical implementation of the above local current scheme. Eventually, the current density tensor is divided by the total conductance or total current to yield normalised current densities that will be presented in Sec. 2.3.

\subsubsection{First principles calculations}

The formalism for calculating currents sketched in the previous section has been applied to the wave functions (2.5) and (2.6) expanded in a basis of TB-MTOs. We here briefly recapitulate some technical aspects of the TB-MTO-WFM method $[45,46]$ that need to be checked in the scattering calculations to determine the dependence of the spin currents and quantities derived from the spin currents.

TB-MTOs are a so-called "first-principles" basis constructed around partial waves, numerical solutions at energy $E$ of the radial Schrödinger equation for potentials that are spherically symmetric inside atomic Wigner-Seitz spheres (AS). The MTOs and matrix elements of the Hamiltonian are constructed from AS potentials calculated self-consistently within the DFT framework combined with short-range "screened structure constants" [51, 74, 75]. Inside an AS, the MTO is expressed as products of partial waves, spherical harmonics and spinors so that a MTO is labelled $|R l m \sigma\rangle$ in the notation of Sec. 2.2.2.

\section{SOC: two and three center terms}

Spin-orbit coupling is included in a perturbative way by adding a Pauli term to the Hamiltonian $[46,50,79,80]$. TB-MTOs lead to a Hamiltonian with one, two and three centre tight-binding-like terms where the three-centre SOC terms introduce longer range hopping [46] than the next-nearest neighbour interaction of 
the "screened structure constant matrix" [51, 74, 75]. Explicit calculation demonstrated that omitting these terms had negligible effect on the resistivity and Gilbert damping but reduced the computational cost by some 70\% [46]. Unless stated otherwise, calculations will only include two center terms.

\section{Partial wave expansion}

In the TB-MTO-WFM code $[45,46]$ the wavefunctions inside atomic spheres are expanded in a partial wave basis that is in principle infinite. In practice the infinite summation must be of course be truncated. For transition metal atoms, we usually use a basis of spd orbitals and test the convergence with an $s p d f$ basis. Unless stated otherwise, an $s p d$ basis will be used.

\section{Scattering configuration: lateral supercells}

Transport in ballistic metals can be studied by constructing an $\mathcal{L}|\mathcal{S}| \mathcal{R}$ scattering configuration with $1 \times 1$ periodicity perpendicular to the transport direction and exploiting the periodicity of the system. Because systems with thermal and chemical disorder or multilayers are not periodic, we model them with a scattering region consisting of a large unit cell transverse to the transport direction that we call a "lateral supercell", Fig. 2.2. Typically this consists of $N \times N$ primitive $1 \times 1$ unit cells containing $M=N^{2}$ atoms. No periodicity is assumed in the transport direction itself that is typically $L$ atomic layers in length $[45,46]$. The size of supercell that can be handled is constrained by computational expense. This scales as the third power of the number of atoms in a lateral supercell and linearly in the length of the scattering region, as $M^{3} L=N^{6} L$. The lateral supercell leads to a reduced twodimensional (2D) Brillouin zone (BZ) and a saving on the BZ sampling so that the computational effort ultimately scales as $M^{2} L=N^{4} L$. An alloy like Py has no longrange order, thus the supercell approximation is only exact for infinite supercell size. In practice, it will turn out that very good results can be obtained for both $\mathrm{Pt}$ and Py using remarkably small lateral supercells.

The simplest way to perform scattering calculations for e.g. thermally disordered Pt is to use ballistic Pt leads. We will examine the effect of a different choice of lead material on the parameter estimates by using other lead materials. The lattice constants of $\mathrm{Au}(a=4.078 \AA)$ and $\mathrm{Ag}(a=4.085 \AA)$ are much closer to that of Pt $(a=3.923 \AA)$ than is that of $\mathrm{Cu}(a=3.615 \AA)$ and by compressing them slightly, they can be made to match Pt without significantly changing their electronic structures. The requirement that leads should have full translational symmetry precludes using an alloy as a lead material [46]. To study the properties of Py ( $a=3.541 \AA$ ), it is convenient to use slightly compressed $\mathrm{Cu}$ as lead material. To use $\mathrm{Cu}$ as a lead for Pt (as mentioned in Sec. 2.1), we constructed a relaxed $\mathrm{Cu}|\mathrm{Pt}| \mathrm{Cu}$ scattering configuration by choosing appropriately matched supercells for 
$\mathrm{Cu}$ and Pt. As long as we are only interested in the bulk properties of Pt and Py, the choice of lead material should not matter; we will demonstrate this explicitly.

\section{Alloy disorder}

Disordered substitutional alloys can be modelled in lateral supercells by randomly populating supercell sites with AS potentials subject to the constraint imposed by the stoichiometry of the targeted experimental system. In principle, the AS potentials can result from self-consistent supercell calculations. In practice, we use the very efficient coherent-potential-approximation (CPA) [54] implemented with TBMTOs [53] to calculate optimal Ni and Fe potentials for Permalloy. Since we will not be studying interface properties in this chapter, we will use CPA potentials calculated for bulk Py rather than using a version of the CPA generalized to allow the optimized potentials to depend on the layer position with respect to an interface [53].

\section{Thermal disorder}

Many experiments in the field of spintronics are performed at room temperature where transport properties are dominated by temperature induced lattice and spin disorder. We will model this type of disorder within the adiabatic approximation using a recently developed "frozen thermal disorder scheme" [66, 67]. In Ref. [67] correlated atomic displacements were determined from the results of lattice dynamics calculations by taking a superposition of phonon modes weighted with a temperature dependent Bose-Einstein occupancy; this was shown to very satisfactorily reproduce earlier results obtained in the lowest order variational approximation (LOVA) with electron phonon matrix elements calculated from first principles with linearized MTOs [64]. Rather than trying to extend this ab-initio approach to disordered alloys, we adopt the simpler procedure of modelling atomic displacements with a Gaussian distribution [66] and choosing the root-mean square displacement $\Delta$ to reproduce the experimental resistivity [67]. Here, it is important to note that $\Delta$ can depend on the choice of orbital basis, supercell size and inclusion of three center terms. For RT Pt, $\Delta$ is chosen to yield the room temperature resistivity $\rho_{\mathrm{Pt}}=10.8 \mu \Omega \mathrm{cm}$ [73]. With this approach, the results we obtain for $l_{\mathrm{sf}}$ and $\Theta_{\mathrm{sH}}$ for RT Pt differ slightly from our earlier work [32, 67]. However, because Pt satisfies the Elliot-Yafet relationship, the products $\rho l_{\mathrm{sf}}$ and $\sigma \Theta_{\mathrm{sH}}$ agree with those earlier publications. Here $\sigma=1 / \rho$ is the conductivity.

Spin disorder is treated analogously [66]. Because spin-wave theory underestimates the temperature induced magnetization reduction, we choose a Gaussian distribution of polar rotations and a uniform distribution in the azimuthal angle to reproduce the temperature dependent magnetization $[46,67]$. The lattice disorder 
is then chosen so that spin and lattice disorder combined reproduce the experimental [81] resistivity of Py, $\rho_{\mathrm{Py}}=15.4 \mu \Omega \mathrm{cm}$, at $300 \mathrm{~K}$.

For both lattice and spin disorder it is necessary to average over a sufficient number of configurations of disorder and to study the effect of the supercell size. All results presented in this chapter are averaged over 20 configurations of disorder.

\section{k-point sampling}

To count all possible scattering states at the Fermi energy a summation over the Bloch wavevectors $\mathbf{k}_{\|}$in the 2D BZ common to the real space supercells must be performed. We sample the $\mathrm{BZ}$ uniformly dividing each reciprocal lattice vector into $Q$ intervals. For an $N \times N$ real space lateral supercell, sampling the 2D BZ with $Q \times Q$ k-points leads to a sampling that is equivalent to an $N Q \times N Q$ sampling for the primitive $1 \times 1$ unit cell.

\section{Slab length}

To extract a value of the SDL characteristic of the bulk, it is important to verify that the decay of the spin current is exponential over a length at least several times longer than $l_{\mathrm{sf}}$ and independent of the lead materials. Because the bulk material is always embedded between two ballistic leads, a deviation from exponential behavior is unavoidable close to the interfaces. We will see that acceptable exponential behaviour is obtained if the lateral supercell and k-space sampling are sufficiently large and the scattering region is sufficiently long.

\section{Averaging $\mathrm{L} \rightarrow \mathrm{R}$ and $\mathrm{R} \rightarrow \mathrm{L}$ currents}

At an interface, the wave character of particles in a quantum mechanical calculation leads to interference between the incident and reflected waves and we observe standing waves in the spin currents that decay away from the interface. These fluctuations are largest close to the left interface for $\mathbf{j}_{s \alpha}^{L R}(z)$ and to the right interface for $\mathbf{j}_{s \alpha}^{R L}(z)$ and gradually disappear towards the other interface, largely paralleling the corresponding unscreened particle accumulations $n^{L R}(z)$ and $n^{R L}(z)$. Even though the oscillations are real effects, we are interested in comparing our data with semiclassical descriptions that do not contain them. For an ideal bulk system, the spin current $\mathbf{j}_{s \alpha}^{L R}(z)$ accompanying a current of electrons from left to right should be identical to the spin current $\mathbf{j}_{s \alpha}^{R L}(z)$ arising from passing a current of holes from right to left. In order to extract various bulk parameters, we use the unscreened particle accumulations $n^{L R}(z)$ and $n^{R L}(z)$ in the following expression to reduce the fluctuations

$$
\mathbf{j}_{s \alpha}^{\mathrm{av}}=\frac{n^{R L}}{n^{L R}+n^{R L}} \mathbf{j}_{s \alpha}^{L R}+\frac{n^{L R}}{n^{L R}+n^{R L}} \mathbf{j}_{s \alpha}^{R L} .
$$

All results presented in this thesis are based upon such averaging. 


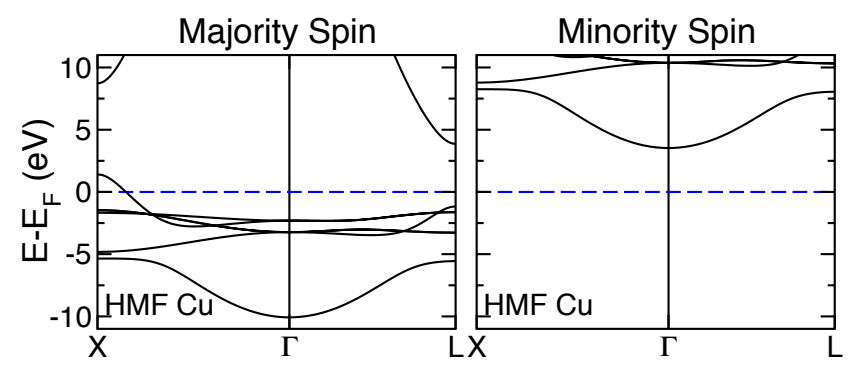

Figure 2.4: Majority (lhs) and minority (rhs) spin band structures of $\mathrm{Cu}$ when a repulsive constant potential of 1 Rydberg is added to the minority spin potential. The effect is to remove all minority spin states from the Fermi energy.

\section{Spin polarized leads}

In order to study the SDL of a material, e.g. Pt, we need to attach magnetic leads to it to inject a spin polarized current. The polarization of a magnetic lead will in general not be unity and the lead|Pt interface will result in a loss of spin signal entering Pt. We maximise the incident spin current by making a halfmetallic ferromagnet (HMF) out of a noble metal. To do so, we add a constant to the potential of one spin channel of the lead material in the scattering calculation to remove that spin channel from the Fermi energy entirely. This is illustrated in Fig. 2.4 where a constant of one Rydberg has been added to the "minority" spin potential to make $\mathrm{Cu}$ HMF. Since we are not interested in interface properties in the present chapter, it is of no concern that this potential is not self-consistent. In a study of real interfaces, more attention would need to be paid to this issue. We denote $\mathrm{Cu}$ made to be HMF in this way as $\mathrm{Cu} \uparrow$.

\subsection{Results}

We illustrate the spin-current formalism with calculations of the SDL $l_{\mathrm{sf}}$ for Pt and Py, the current polarization $\beta$ for Py and the spin Hall angle $\Theta_{\mathrm{sH}}$ for Pt, all at room temperature. The words spin currents and spin current densities will be used interchangeably. Because the results of calculations are always presented in terms of spin current densities normalized with respect to the constant total current $j \equiv j_{c}^{z}(z)$ in the $z$ direction, we omit the over $j_{s}(z)$ in (2.3) when there is no ambiguity.

\subsection{1 $l_{\mathrm{sf}}$ for Pt}

We inject a fully polarized current from a HMF ballistic Au个 lead into RT thermally disordered Pt along the $z$-axis chosen to be the fcc (111) direction perpendicular to close packed atomic layers with the spin current polarized along the $z$-axis. The 


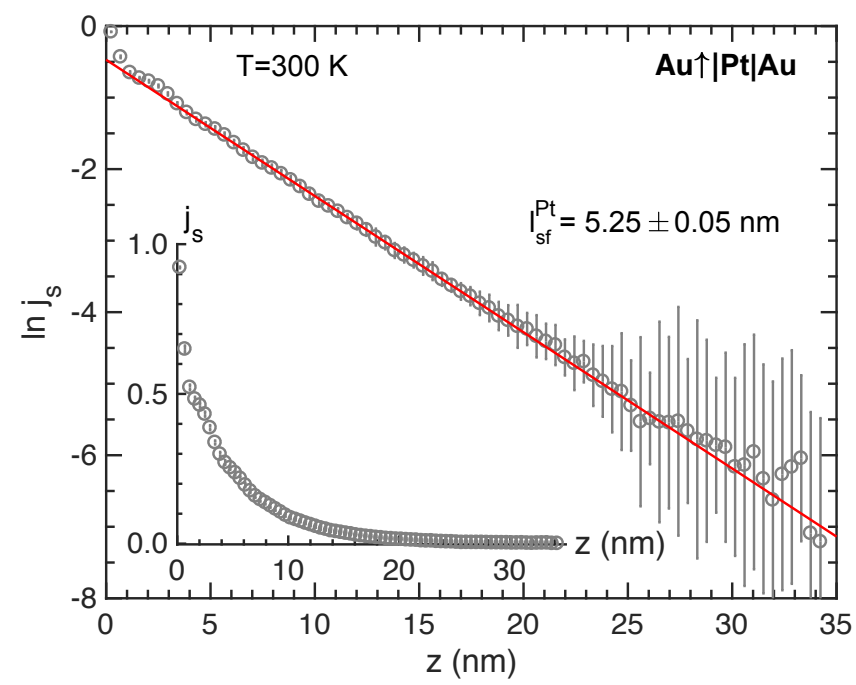

Figure 2.5: Natural logarithm of the spin current injected into RT Pt as a function of the coordinate $z$ in the transport direction. The inset shows the spin current on a linear scale. The current was extracted from the results of a scattering calculation for a two-terminal Au个|Pt|Au configuration using a $7 \times 7$ lateral supercell. The red line is a weighted linear least squares fit; the error bar in the value $5.25 \pm 0.05$ results from different "reasonable" weightings and cutoff values.

distribution of the random displacments of the Pt atoms from their equilibrium lattice positions is Gaussian with a rms displacement $\Delta$ chosen to reproduce the experimental RT resistivity.

The natural logarithm of $j_{s}(z)$ is shown in Fig. 2.5. In the linear plot shown in the inset, we see an initial rapid decrease of the spin current over a distance of order $1 \mathrm{~nm}$ from a value close to unity at the interface, followed by oscillatory damped behaviour that rapidly decays to 0 . The exponential decay over almost five orders is very clear in the logarithmic plot. The red line is a weighted linear least squares fit to (2.2) from which data up to $4 \mathrm{~nm}$ are excluded (including the interface and first half cycle of the oscillatory term). The slope directly yields a value of $l_{\mathrm{sf}}^{\mathrm{Pt}}=5.25 \pm 0.05 \mathrm{~nm}$. The weights are selected to be the inverse of the variance of the spin currents that results from 20 different configurations of thermal disorder. The error bar is then estimated using weighted residuals.

The initial decrease at the interface of $\sim e^{-\frac{1}{2}}$ over a length of $z=1 \mathrm{~nm}$ leads directly to an "interface" $l_{\mathrm{sf}}^{I} \sim 2 \mathrm{~nm}$. Using the definition $[16,82,83]$ of the interface "spin memory loss" parameter $\delta=t_{I} / l_{\mathrm{sf}}^{I}$ in terms of an interface thickness $t_{I}=1 \mathrm{~nm}$ yields a value of $\delta \sim 0.5$, a reasonable value [10]. The clearly visible oscillations in the spin current are not predicted by semiclassical treatments. We attribute them to Fermi surface nesting-like features but more analysis would be 


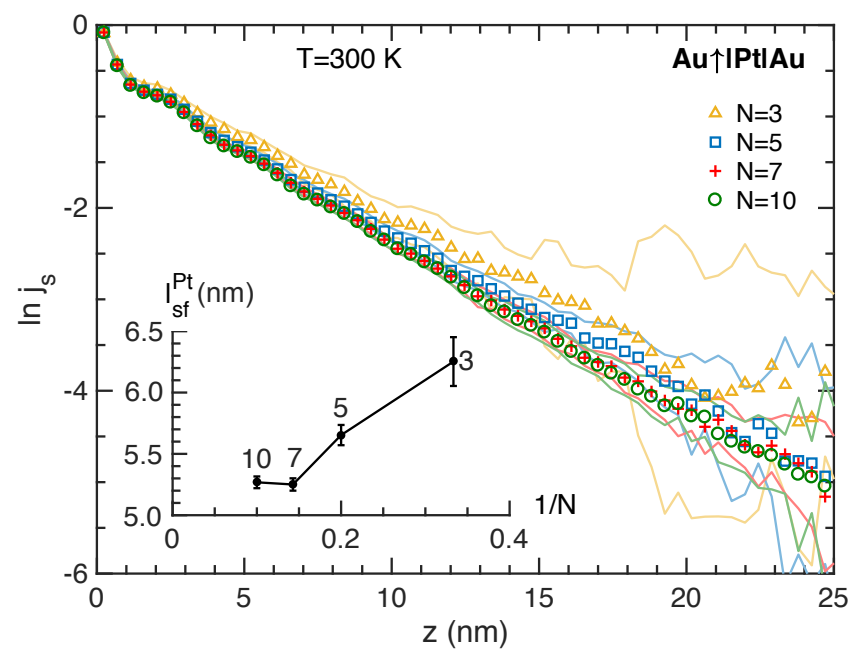

Figure 2.6: Natural logarithm of the spin current density vs length for various $N \times N$ supercells $(N=3,5,7,10)$ for a $35 \mathrm{~nm}$ long Pt slab at $300 \mathrm{~K}$. The color coordinated symbols and solid lines indicate the mean and a measure of the spread of the data from 20 different configurations, respectively. Inset: SDL obtained from the linear fit of $\ln j_{s}(z)$ shown as a function of $1 / N$. The numerical values of $l_{\mathrm{sf}}$ are given in Table 2.1.

required to establish this firmly.

The results shown in Fig. 2.5 were calculated in a $7 \times 7$ Pt lateral supercell with an $s p d$ basis and using a $2 \mathrm{D}$ BZ sampling of $32 \times 32 \mathrm{k}$ points equivalent to a $224 \times 224$ sampling for a $1 \times 1$ unit cell. In the remainder of this section we will examine how $l_{\mathrm{sf}}^{\mathrm{Pt}}$ depends on these and a number of the other computational parameters discussed in Sect. 2.2.5.

\section{Supercell size}

It is not a priori clear how large a lateral supercell should be in order to adequately represent diffusive transport. On the one hand, one might expect it should be larger than the mean free path; in that case, this project would be doomed to failure for all but the most resistive of materials. On the other hand, only electrons scattered through $90^{\circ}$ "know" about the lateral translational symmetry. In Fig. 2.6, we show the natural logarithm of the normalized spin current density calculated for a Au个|Pt|Au scattering geometry using Pt $N \times N$ lateral supercells with $N=$ 3, 5, 7, 10; the largest supercell contains some 15000 atoms. For each value of $N$, we choose the BZ k-sampling parameter $Q$ so that $N Q \sim 160$ in order to maintain a constant reciprocal space sampling equivalent to $160 \times 160$ for a $1 \times 1$ primitive unit cell. The main features seen in Fig. 2.5 are reproduced for all values of $N$. 
Table 2.1: Dependence of the calculated SDL of RT Pt on the $N \times N$ supercell size for $N=3,5,7,10$. Calculations were performed with a k-point sampling equivalent to $160 \times 160$ for a $1 \times 1$ supercell in each case.

\begin{tabular}{rccc}
\hline \hline$N$ & $s p d+2$ center & $s p d+3$ center & $s p d f+2$ center \\
\hline 3 & $6.25 \pm 0.20$ & & \\
5 & $5.65 \pm 0.08$ & $5.22 \pm 0.09$ & $5.21 \pm 0.07$ \\
7 & $5.25 \pm 0.05$ & $4.96 \pm 0.07$ & \\
10 & $5.27 \pm 0.05$ & & \\
\hline \hline
\end{tabular}

The most important trend is that $l_{\mathrm{sf}}$ decreases slightly with increasing $N$. As seen clearly in the inset, it converges rapidly to a value of $\sim 5.25 \mathrm{~nm}$; the values are given separately in Table 2.1. For room temperature Pt, we see that it is sufficient to use a $7 \times 7$ supercell.

Perhaps more striking is how rapidly the error bar decreases; see the inset. This can be easily understood. In an $N \times N$ lateral supercell, a single configuration of disorder "seen" by a spin before it flips contains of order $N^{2} l_{\mathrm{sf}} / d$ atoms where $d$ is the spacing between Pt (111) planes $\sim 0.2 \mathrm{~nm}$. For $N=3$, this amounts to only about 250 atoms, for $N=10$, it is about 2500 . For short values of $l_{\mathrm{sf}}$ or small lateral supercells, we expect very large configuration to configuration variation and to have to include more configurations of disorder in our configuration averaging. By itself, this will not be sufficient because the freedom available to sample thermal disorder in a small supercell is intrinsically limited e.g. long wavelength transverse fluctuations cannot be represented in small supercells.

This has another important consequence. If we assume that a Au个 lead has a single scattering state per $\mathbf{k}_{\|}$point in a $1 \times 1$ primitive interface unit cell, this means we begin with $160 \times 160=25600$ states incident on the scattering region. For $z=6 l_{\mathrm{sf}}=31 \mathrm{~nm}, e^{-6} \sim=\frac{1}{400}$. Of the 25600 scattering states we started with in the left hand lead, we lose half at the interface and eventually only about 32 states are transmitted into the right hand lead without flipping their spins. This accounts for the large amount of noise seen in the spin current density for large values of $z$. This can be reduced to some extent by increasing the number of $\mathrm{k}$ points used to sample the BZ but is ultimately limited by a too-small supercell size.

\section{k-point sampling}

The last point brings us to the question of BZ sampling. The spin current $j_{s}(z)$ is obtained by summing partial spin currents over a discrete grid of $\mathbf{k}_{\|}$vectors in a $2 \mathrm{D} \mathrm{BZ}$ and integrating over $x y$ planes of real space atomic layers. As the BZ grid becomes finer, the fluctuations in spin current density in each layer must tend towards a converged value dependent on the lateral supercell size. In Fig. 2.7 we 


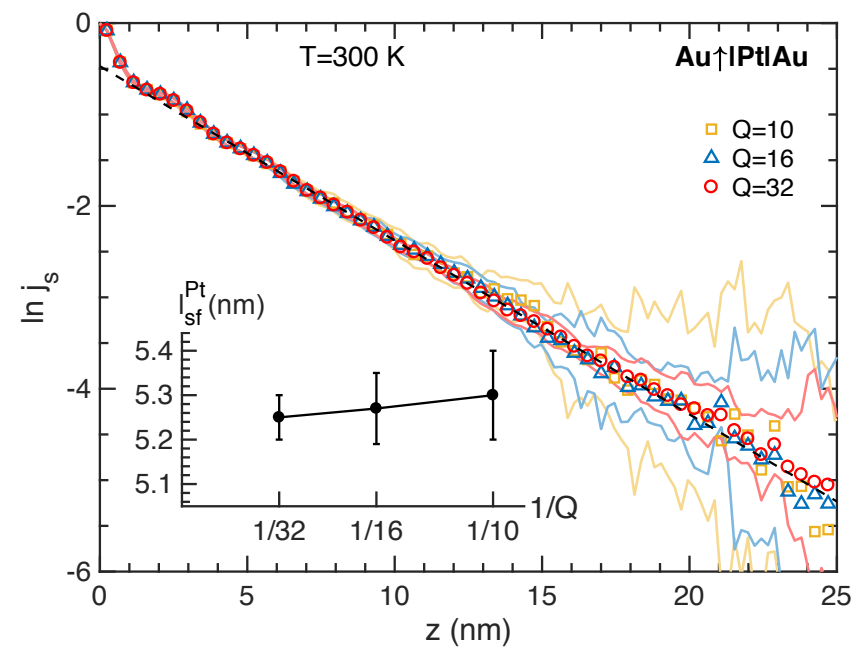

Figure 2.7: Natural logarithm of the spin current density calculated for RT Pt with a 7×7 lateral supercell and three different $Q \times Q$ samplings of the BZ: $Q=10$ (yellow squares), $Q=16$ (blue triangles) and $Q=32$ (red circles). The color coordinated symbols and solid lines indicate the mean and a measure of the spread of the data, respectively, for 20 different configurations for different $\mathrm{Q}$ samplings. The dashed black line indicates the linear fit determined for $\ln j_{s}$ calculated with $Q=32$. Though the three curves initially overlap perfectly, for $z \geq 14 \mathrm{~nm}$ we see that noise in the mean and spread of $\ln j_{s}$ for $Q=10$ is substantially larger than for the $Q=32$ data. Inset: $l_{\mathrm{sf}}^{\mathrm{Pt}}$ as a function of the BZ sampling parameter $Q$.

show the fluctuations found as a function of $z$ for a room temperature Pt slab of length $\sim 35 \mathrm{~nm}$ and an $N=7$ lateral supercell. We compare the results obtained for three $Q \times Q$ BZ sampling densities with $Q=10,16,32$. As the spin currents become smaller, the noise in the data becomes larger. The solid lines in Fig. 2.7 are a measure of the spread found for 20 random configurations of disorder. The spread becomes significantly smaller with increasing $Q$. Since the current injected from the left lead is fully polarized, the noise does not significantly affect the determination of $l_{\mathrm{sf}}^{\mathrm{Pt}}$. We shall see in the next subsection that a smaller spin current entering from a diffuse Py|Pt interface leads to more noise in the data, making the choice of BZ sampling more critical.

\section{Leads}

In Fig. 2.1 we showed how $l_{\text {sf }}$ obtained directly from the transmission matrix depended on the choice of lead material. Here we demonstrate that when determined from the decay of the spin current, $l_{\mathrm{sf}}$ does not depend on the lead material used. To study this, we carried out calculations for a $35 \mathrm{~nm}$ long slab of RT Pt with a 


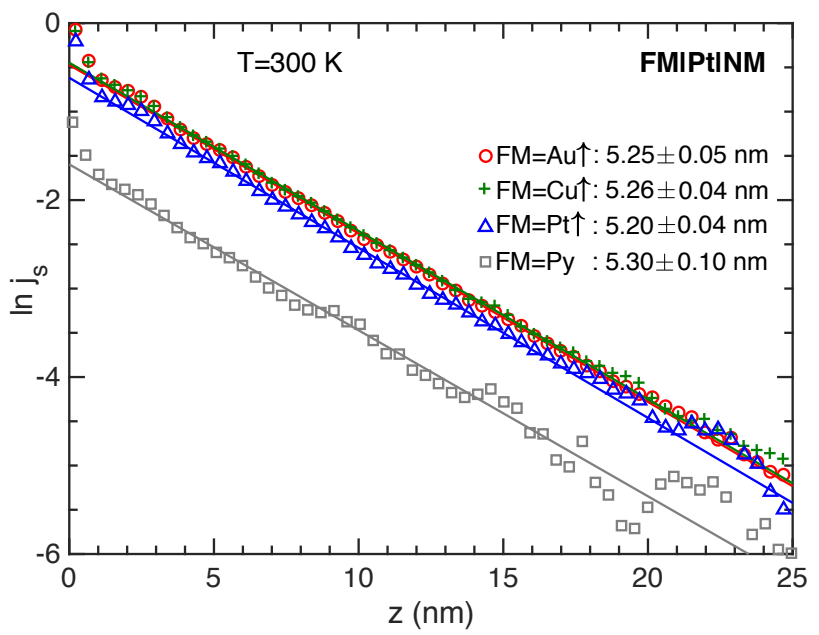

Figure 2.8: Natural logarithm of the spin current injected into Pt as a function of the coordinate in the transport direction $z$ using different lead materials. The current was extracted from the results of a scattering calculation for a two-terminal $\mathrm{NM} \uparrow|\mathrm{Pt}| \mathrm{NM}^{\prime}$ configuration. The lattice constant of the Au leads was scaled to match to $\mathrm{Pt}$. In the case of $\mathrm{Cu}$, a lateral $\mathrm{Cu} 8 \times 8$ supercell was matched to a $2 \sqrt{13} \times$ $2 \sqrt{13}$ lateral Pt supercell. Injection from permalloy was realized in a $\mathrm{Cu}|\mathrm{Py}| \mathrm{Pt} \mid \mathrm{Cu}$ configuration where lattice matching was realized using the same supercells as for $\mathrm{Cu}|\mathrm{Pt}| \mathrm{Cu}$. The straight lines are weighted linear least squares fits from which the interface region is omitted; the error bars result from different "reasonable" weightings and cutoff values.

$7 \times 7$ lateral supercell and a $32 \times 32 \mathrm{BZ}$ sampling for three different lead materials: ballistic $\mathrm{HMF} \mathrm{Cu} \uparrow, \mathrm{Au} \uparrow$ and $\mathrm{Pt} \uparrow$ leads, in each case raising the spin-down electronic bands above the Fermi energy by adding a constant to the AS potential. Thus, a fully polarized spin current enters Pt and decays exponentially as shown in Fig. 2.8.

To within $1 \%, l_{\mathrm{sf}}^{\mathrm{Pt}}$ is the same for all lead materials. The quantum oscillations are also independent of the lead material supporting our assertion that they are an intrinsic property of Pt. What does change is the interface contribution to the loss of spin current ("spin memory loss") as indicated by different intercepts for the three different HMF leads.

Since these leads were polarized artificially, we also examine what happens when a "naturally" polarized current from a ferromagnetic material enters Pt. We used an $8 \times 8$ lateral supercell of $\mathrm{Cu} \mid \mathrm{Py}$ to match to a $2 \sqrt{13} \times 2 \sqrt{13}$ lateral supercell of Pt and absorbed the residual mismatch in a small trigonal distortion of Pt. For this geometry, we find $l_{\mathrm{sf}}^{\mathrm{Pt}}=5.3 \pm 0.1 \mathrm{~nm}$ in Fig. 2.8 (grey squares). The slight difference from the other values can be traced to the small trigonal distortion of the Pt lattice. Compared to the HMF lead cases, a smaller spin current enters Pt 
from Py because (i) the current polarization in Py is not 100\% (see Sect. 2.3.2) and (ii) because of the spin-flipping at the interface (spin-memory loss). As discussed in the previous subsection, the noise in $\ln j_{s}(z)$ for smaller absolute values of $j_{s}(z)$ at large $z$ could be reduced somewhat by increasing the BZ sampling.

\section{Three center terms}

Including three center terms in the SOC part of the Hamiltonian more than triples the computational cost [46]. The effect on $l_{\mathrm{sf}}^{\mathrm{Pt}}$ is compared for $5 \times 5$ and $7 \times 7$ supercells in Table 2.1. For a $5 \times 5$ supercell we find that $l_{\mathrm{P} t}$ decreases by $7.5 \%$ from $5.65 \pm 0.08 \mathrm{~nm}$ with two center terms to $5.22 \pm 0.09 \mathrm{~nm}$ with three center terms. For a $7 \times 7$ supercell, $l_{\mathrm{sf}}^{\mathrm{Pt}}$ decreases by $5.5 \%$ from $5.25 \pm 0.05 \mathrm{~nm}$ with two center terms to $4.96 \pm 0.07 \mathrm{~nm}$ with three center terms.

\section{Basis: spd vs spdf}

Using a 16 orbital $s p d f$ basis instead of a 9 orbital $s p d$ basis increases the computational costs by a factor $(16 / 9)^{3} \sim 5.6$. Thus, we use only a $5 \times 5$ lateral supercell to estimate the effect of including $f$ orbitals on $l_{\text {sf }}^{\mathrm{Pt}}$ compared with the $s p d$ results in Table 2.1. We find a 7.5\% decrease in $l_{\mathrm{sf}}^{\mathrm{Pt}}$ from $5.65 \pm 0.08 \mathrm{~nm}$ with an $s p d$ basis to $5.21 \pm 0.07 \mathrm{~nm}$ with an $s p d f$ basis. In view of the substantial computational costs incurred in including them and their relatively small effect on $l_{\mathrm{sf}}$, neglect of the three centre terms in the Hamiltonian and $f$ orbitals in the basis is justified by the much larger uncertainty that currently exists in the experimental determination of $l_{\mathrm{sf}}$. The only barrier to including them, should the experimental situation warrant an improved estimate, is computational expense. Our best estimate of $l_{\mathrm{sf}}^{\mathrm{Pt}}$ at $300 \mathrm{~K}$ is $5.3 \pm 0.4 \mathrm{~nm}$.

\subsection{2 $\beta$ for Permalloy}

To determine the transport polarization $\beta$ of Py, we can use a symmetric NM|FM|NM configuration and equation (2.3). By choosing the center of the FM slab to be the origin $z=0$ with the interfaces at $z= \pm L / 2, \widehat{j_{s}}(z)=\beta-c \cosh \left(z / l_{\mathrm{sf}}\right)$ as in (2.3). The results of injecting an unpolarized current from $\mathrm{Cu}$ leads into a $40 \mathrm{~nm}$ thick slab of RT Py are shown in Fig. 2.9(a) for a $5 \times 5$ lateral supercell. $\beta$ and $l_{\mathrm{sf}}^{\mathrm{Py}}$ are determined simultaneously by using both as free parameters for the fitting.

Since the current polarization for an infinitely long Py slab should be $\beta$ for all $z$ and because $\cosh (0)=1, c$ must vanish in the limit $L \rightarrow \infty$ over a length scale $l_{\mathrm{sf}}$. Because the scattering region is finite in length, $\widehat{j_{s}}(z)$ must be fitted to $\beta-c(L) \cosh \left(z / l_{\mathrm{sf}}\right)$. We need to determine $c(L)$ or ensure that it does not affect the values of $\beta$ and $l_{\mathrm{sf}}$ obtained by fitting. These values are given in Table 2.2 as a 


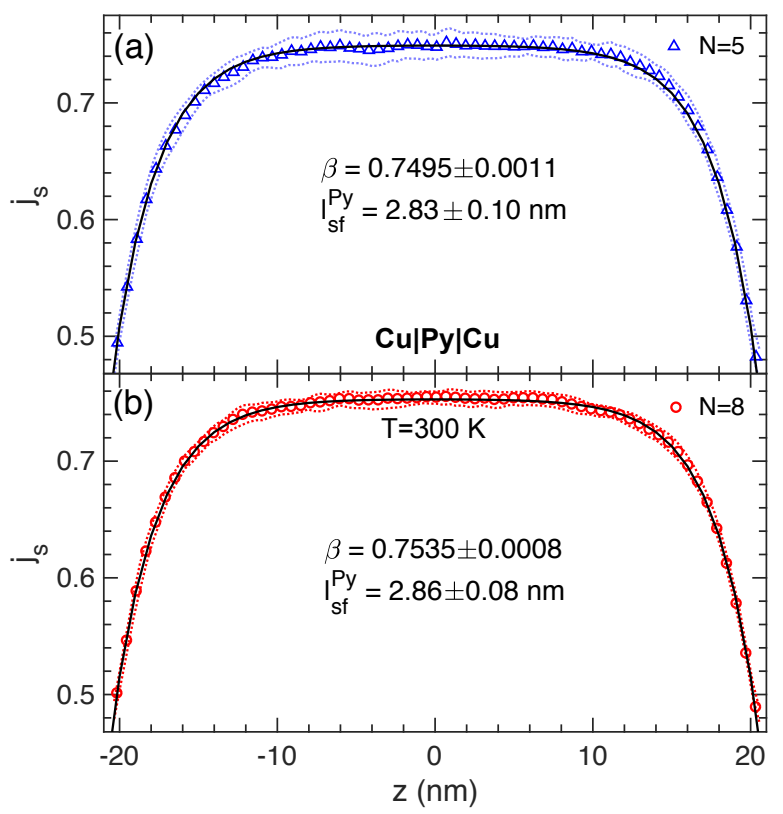

Figure 2.9: A charge current passed through Py polarizes to $\beta$ in the middle of the scattering region. By fitting (solid black lines) the spin current calculated for an $N \times N$ supercell to Eq. (2.3), $\beta$ and $l_{\text {sf }}^{\mathrm{Py}}$ are extracted for (a) $N=5$ (blue) and (b) $N=8$ (red). The dotted lines indicate the spread from 20 different configurations of disorder.

function of the length of the scattering region, $L_{\mathrm{Py}} \simeq 10,20,30,40 \mathrm{~nm}$. Reasonable estimates of $l_{\mathrm{sf}}$ and $\beta$ are found for $L \geq 20 \mathrm{~nm}$ with very acceptable error bars.

\section{Supercell size}

We compare the results obtained for Py with $5 \times 5$ and $8 \times 8$ supercells in Fig. 2.9 and Table 2.2. Both thermal and chemical disorder contribute to the fluctuations which are larger for $N=5$ than for $N=8$. However, the parameter estimates do not show a significant $N$ dependence. Carrying out a calculation with $N=8$ would be necessary only in cases where statistical fluctuations or parameter errors are unacceptably large.

\section{Averaging $\mathrm{L} \rightarrow \mathrm{R}$ and $\mathrm{R} \rightarrow \mathrm{L}$ currents}

In Fig. 2.10, we plot the spin current $j_{s z}^{L R}(z)$ that arises when a current of electrons is passed from left to right, and $j_{s z}^{R L}(z)$ when a current of holes is passed from right to left, for a $40 \mathrm{~nm}$ long slab of an $5 \times 5$ supercell of Py sandwiched between ballistic $\mathrm{Cu}$ leads. Reflections at the $\mathrm{Cu} \mid \mathrm{Py}$ interfaces give rise to interferences that slowly 


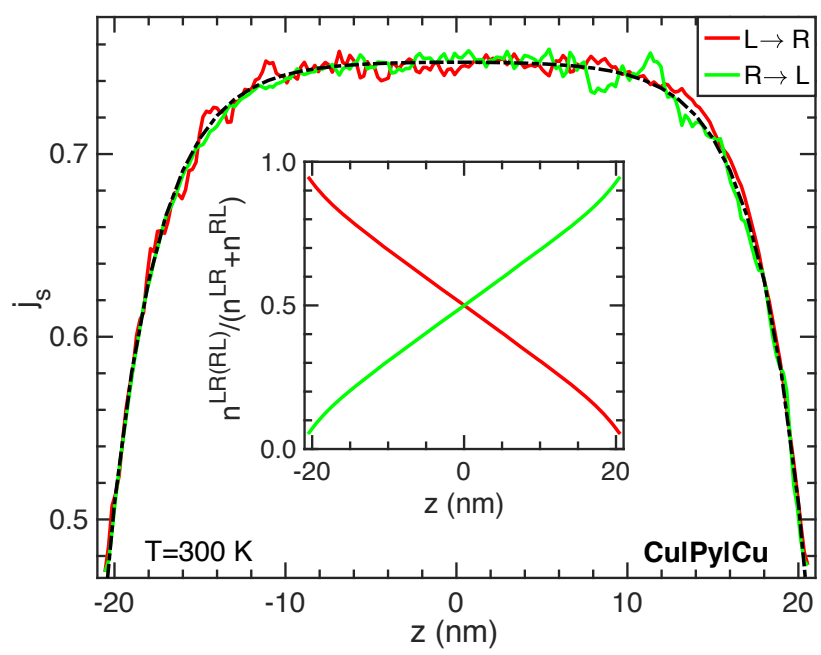

Figure 2.10: Spin currents $\widehat{j}_{s z}(z)$ obtained by injecting electrons into Py from a left $\mathrm{Cu}$ lead (red) and from injecting holes from a right $\mathrm{Cu}$ lead (green) for an $5 \times 5$ lateral supercell. The black dashed line shows the fit to the averaged current discussed in the text. Inset: Fractional nonequilibrium particle densities incident from left (red) and right (green) leads.

decay into the scattering region. The interference and its decay are clearly visible in Fig. 2.10 for both currents as they progress from the source to the drain lead. The fluctuations are significantly reduced after averaging using (2.25).

\subsection{3 $l_{\mathrm{sf}}$ for Permalloy}

Although we obtain reasonable values for $l_{\mathrm{sf}}^{\mathrm{Py}}$ simultaneously with $\beta$, it can be desirable to be able to extract $l_{\mathrm{sf}}^{\mathrm{Py}}$ independently. For a symmetric NM|FM|NM configuration, the spin current has the form (2.2) which approaches $\beta$ asymptotically for scattering regions much longer than $l_{\mathrm{sf}}$. Unlike $l_{\mathrm{sf}}^{\mathrm{Pt}}$ for which $\beta=0$, the finite asymptotic value of $\beta$ prevents us from extracting $l_{\mathrm{sf}}^{\mathrm{Py}}$ by taking the logarithm of $j_{s}(z)$. However, by considering $\mathrm{NM} \uparrow|\mathrm{FM}| \mathrm{NM}$ and $\mathrm{NM} \downarrow|\mathrm{FM}| \mathrm{NM}$ configurations for which $j_{s}^{\uparrow}(z)=\beta+b_{\uparrow} e^{-z / l_{\mathrm{sf}}}-a_{\uparrow} e^{z / l_{\mathrm{sf}}}$ and, respectively, $j_{s}^{\downarrow}(z)=\beta+b_{\downarrow} e^{-z / l_{\mathrm{sf}}}-a_{\downarrow} e^{z / l_{\mathrm{sf}}}$, we can take the difference so the constant $\beta$ drops out. We then consider a long scattering region and values of $z$ far from the right-hand interface so that the exponentially increasing terms can be neglected and we are left with a pure exponentially decreasing function. To optimize the "systematic cancellation" when taking the difference of the two spin currents, we use identical microscopic configurations of alloy and thermal disorder to perform scattering calculations first with $\mathrm{Cu} \uparrow$ and then with $\mathrm{Cu} \downarrow$ left leads. This is then done pairwise for 20 different configurations 


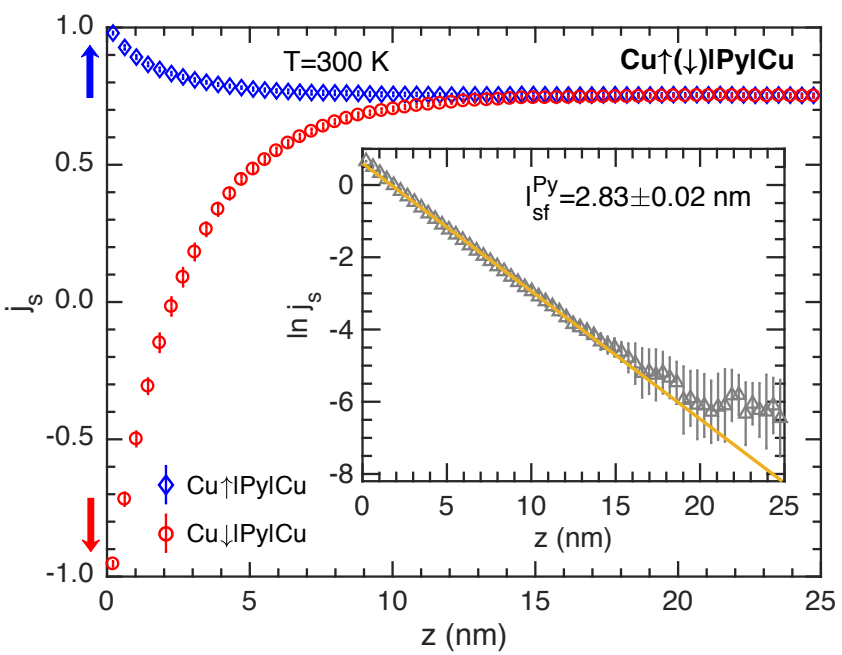

Figure 2.11: Fully polarized spin-up (blue) and spin-down (red) currents injected from the left ballistic $\mathrm{Cu} \uparrow$, respectively $\mathrm{Cu} \downarrow$ lead into $40 \mathrm{~nm}$ of Py with RT thermal lattice and spin disorder saturate to $\beta$ far from the lead. The difference of the two currents decays exponentially to zero. The supercell size is $8 \times 8$ and the Brillouin zone k-sampling is $28 \times 28$. Inset: natural $\log$ of the difference is fit linearly (in yellow) to yield $l_{\mathrm{Py}}=2.83 \pm 0.02 \mathrm{~nm}$.

of $40 \mathrm{~nm}$ long disordered Py to obtain the results shown in Fig. 2.11. The small oscillations in the spin current found for Pt are not observed here for Py. Presumably, this is due to the larger amount of disorder, as we now also have thermal spin disorder and substitutional alloy disorder in addition to the thermal lattice disorder, resulting in a pure exponential decay of $j_{s}^{\uparrow}(z)-j_{s}^{\downarrow}(z)$.

The natural logarithm of the difference is shown in the inset to Fig. 2.11. A weighted linear least squares fit yields a room temperature decay length of $l_{\mathrm{sf}}^{\mathrm{Py}}=$ $2.83 \pm 0.02 \mathrm{~nm}$ for $8 \times 8$ supercell. Only data between $z=2$ and $20 \mathrm{~nm}$ is used for the fitting. The small curvature around $z=0 \mathrm{~nm}$ is due to spin-memory loss. Beyond $z=20 \mathrm{~nm}$ the variance in the spin current is relatively larger, and the exponentially increasing term in $j_{s}^{\uparrow}(z)-j_{s}^{\downarrow}(z)$ is not negligible. Since the region of fitting is $\sim 6 l_{\mathrm{sf}}^{\mathrm{Py}}$, these effects are of little consequence. The weights are selected to be the inverse of the variance of the spin currents due to different configurations. The error bar is then estimated using weighted residuals. It is worth emphasizing that the value of $l_{\mathrm{sf}}^{\mathrm{Py}}=2.83 \pm 0.02 \mathrm{~nm}$ obtained using HMF leads is in perfect agreement with the value $l_{\mathrm{sf}}^{\mathrm{Py}}=2.86 \pm 0.08$ (Fig. 2.9 and Table 2.2) obtained by passing a current from unpolarized NM leads. For Py at room temperature, our best estimate of $l_{\mathrm{sf}}^{\mathrm{Py}}$ is $2.8 \pm 0.1 \mathrm{~nm}$ and of $\beta$ is $0.75 \pm 0.01$. 
Table 2.2: Dependence of the SDL and polarization $\beta$ on the length $L_{\mathrm{Py}}$ of the Py slab and on the $N \times N$ supercell size for Py at $300 \mathrm{~K}$ for $N=5,8$. Calculations are performed with k-point sampling equivalent to $140 \times 140$ for a $1 \times 1$ unit cell in each case.

\begin{tabular}{cccc}
\hline \hline$N$ & $L_{\mathrm{Py}}(\mathrm{nm})$ & $l_{\text {sf }}(\mathrm{nm})$ & $\beta$ \\
\hline 5 & 10.44 & $2.29 \pm 0.72$ & $0.7200 \pm 0.0500$ \\
& 20.66 & $2.71 \pm 0.14$ & $0.7410 \pm 0.0032$ \\
& 30.88 & $2.69 \pm 0.08$ & $0.7481 \pm 0.0014$ \\
& 41.11 & $2.83 \pm 0.10$ & $0.7495 \pm 0.0011$ \\
\hline 8 & 41.11 & $2.86 \pm 0.08$ & $0.7535 \pm 0.0007$ \\
\hline \hline
\end{tabular}

\subsubsection{Spin Hall angle for Platinum}

A charge current passed through a length of diffusive Pt sandwiched between ballistic Pt leads results in spin currents in the transverse direction; this is the spin Hall effect $[11,12,18-20]$. The polarization direction of the spin current is given by a vector product of the original current direction $(z)$ and the transverse spin current direction. Thus, spin currents in the $x$ and $-y$ directions are polarized in the $y$ and $x$ directions, respectively and have the same amplitude reflecting the axial symmetry of the system. These two transverse currents normalized to the longitudinal charge current, $\widehat{j}_{s y}^{x}(z)$ and $-\widehat{j_{s x}^{y}}(z)$, are plotted in Fig. 2.12(a) for a RT (111) oriented slab of Pt. The fluctuations about the bulk value are a result of a combination of configuration averaging, supercell size and BZ sampling.

We extract the bulk value of the spin-Hall angle $\Theta_{\mathrm{sH}}$ as follows. Starting from the left interface at $z=0$, the configuration average of $\widehat{j}_{s y}^{x}(z)$ and $\widehat{j}_{s x}^{y}(z)$ is integrated over atomic layers up to some $L_{\mathrm{Pt}}: J_{s}^{\perp}\left(L_{\mathrm{Pt}}\right)=\int_{0}^{L_{\mathrm{Pt}}}\left[\widehat{j}_{s y}^{x}(z)-\widehat{j}_{s x}^{y}(z)\right] / 2 d z$. The integrated quantities for a number of discrete values of $L_{\mathrm{Pt}}$ are shown in the inset to Fig. 2.12(a) as green stars. A least squares fit to linear behaviour yields a value of $\Theta_{\mathrm{sH}}=3.71 \pm 0.03 \%$ as the slope [32]. The error bar results from the weighted residuals where the weights are the mean deviation for 20 configurations of thermal disorder.

The above calculations were carried out with a $7 \times 7$ lateral supercell and a $22 \times 22 \mathrm{BZ}$ sampling that is equivalent to a $154 \times 154$ sampling for a $1 \times 1$ unit cell. We now examine the effect on $\Theta_{\mathrm{sH}}$ of varying some of the different computational parameters discussed in Sec. 2.2.5.

\section{Leads}

To rule out an eventual dependence of $\Theta_{\mathrm{sH}}$ on the leads, results for Pt and Au leads are compared in Figs. 2.12(a) and (b) respectively. Close to the Au leads, the 

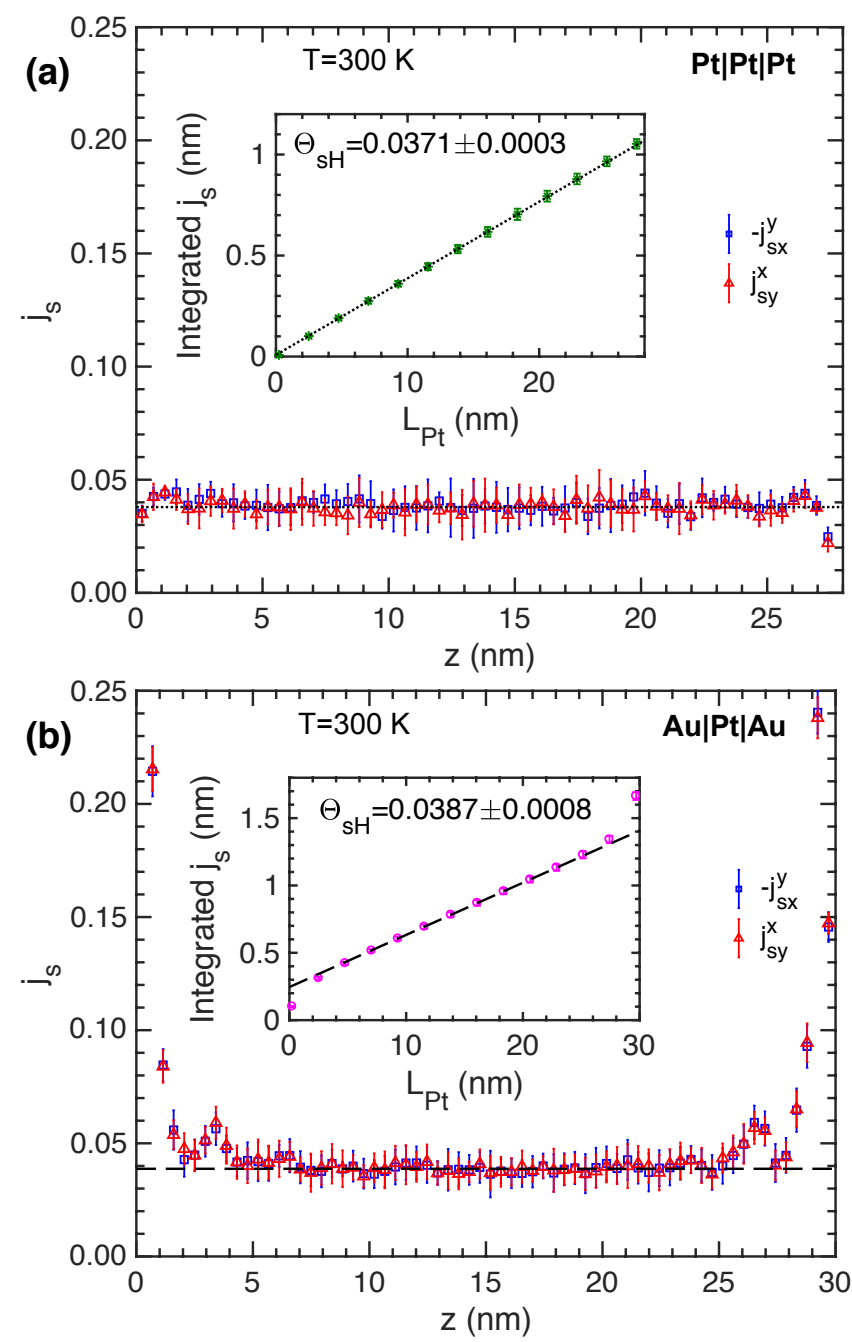

Figure 2.12: Transverse spin currents driven by a charge current in the $z$ direction for a (111) oriented Pt slab embedded between (a) Pt and (b) Au leads. The error bars are the mean deviation of the currents for 20 configurations of disorder. The horizontal dotted and dashed lines indicate the extracted values of $\Theta_{\mathrm{sH}}$. For both leads, $7 \times 7$ supercells were used with a $22 \times 22 \mathrm{BZ}$ sampling. Inset: Integrated transverse spin currents as a function of $L_{\mathrm{Pt}}$ for a RT diffusive Pt scattering region embedded between ballistic Pt (green stars) (a) and Au (pink circles) (b) leads. The dotted and dashed lines indicate the weighted linear least squares fit with Pt (a) and Au (b) leads, respectively. The interface contributions in (a) are negligible compared to (b). 
transverse spin currents are dominated by a huge Au|Pt interface contribution [32] and then drop rapidly towards the bulk value, indicated by the horizontal dashed line, away from the two interfaces. The interface contributions with Pt leads are negligible compared to Au. The slopes determined by linear least squares fitting of the integrated spin current density are nearly identical. To ensure a sufficiently long range in $L_{\mathrm{Pt}}$ that exhibits linear behaviour, a longer length of Pt must be used with Au leads than with Pt leads.

\section{Supercell size and k-point sampling}

We studied how the SHA depends on the size of the lateral supercell with $N=$ $3,5,7,10$ using a BZ sampling $Q$ for each $N$ that corresponds to sampling a $1 \times 1$ unit cell with $N Q \sim 160 \mathrm{k}$ points. Unlike $l_{\mathrm{sf}}^{\mathrm{Pt}}, \Theta_{\mathrm{sH}}$ shows a negligible dependence on the supercell size, as seen in Table 2.3 for results calculated with two center SOC terms. On changing $N$, the central value scarcely changes with respect to the value $\Theta_{\mathrm{sH}}=3.71 \%$ found above. What does change is that the already small error bar decreases with increasing supercell size.

Calculating $\Theta_{\mathrm{sH}}$ for a 7 $\times 7$ Pt supercell with a denser k-sampling, $Q=32(N Q=$ 224), yields $\Theta_{\mathrm{sH}}=3.73 \%$ compared to $\Theta_{\mathrm{sH}}=3.71 \%$ with $Q=22(N Q=154)$, see Table 2.3. Thus a choice of $Q=22$ for $N=7$ is quite sufficient.

\section{SOC: three center terms}

The results obtained with the three center terms in the SOC Hamiltonian included are also given in Table 2.3. $\Theta_{\mathrm{sH}}$ increases by about a third compared to the values with two center terms. Three center (3C) terms are thus seen to affect $\Theta_{\mathrm{sH}}$ much more than $l_{\mathrm{sf}}^{\mathrm{Pt}}$. We return to this below.

Table 2.3: Dependence of $\Theta_{\mathrm{sH}}$ (in \%) for RT Pt on the supercell size $N$, without and with three centre SOC terms. Calculations were performed with a $Q \times Q$ kpoint sampling nearly equivalent to $160 \times 160$ for a $1 \times 1$ supercell in each case; $N \times Q \sim 160$.

\begin{tabular}{|c|c|c|c|c|c|}
\hline \multirow[b]{2}{*}{$N$} & \multirow[b]{2}{*}{$Q$} & \multirow[b]{2}{*}{$N \times Q$} & \multicolumn{2}{|c|}{ spd } & \multirow{2}{*}{$\frac{s p d f}{2 \text { center }}$} \\
\hline & & & 2 center & 3 center & \\
\hline 3 & 54 & 162 & $3.65 \pm 0.07$ & & \\
\hline 5 & 32 & 160 & $3.79 \pm 0.06$ & $5.1 \pm 0.2$ & $2.95 \pm 0.03$ \\
\hline 7 & 22 & 154 & $3.71 \pm 0.03$ & $5.0 \pm 0.1$ & \\
\hline 7 & 32 & 224 & $3.73 \pm 0.03$ & & \\
\hline 10 & 16 & 160 & $3.75 \pm 0.01$ & & \\
\hline
\end{tabular}




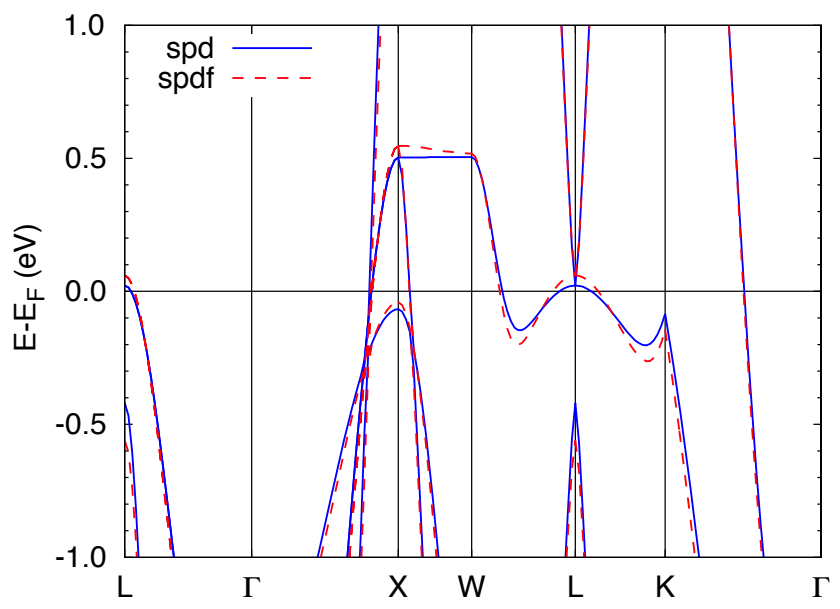

Figure 2.13: Band structure of Pt evaluated using Stuttgart LMTO code with an spd (blue) and $s p d f$ (red) basis. The dispersion about the Fermi energy and details of the Fermi surface are very sensitive to the choice of basis.

\section{Basis: spd vs spdf}

Augmenting the spd basis with $f$ orbitals increases the computational effort by a factor $(16 / 9)^{3} \sim 5.6$ and reduces $\Theta_{\mathrm{sH}}$ by about a fifth from $3.71 \%$ to $2.95 \%$. The sensitivity of the Pt spin-Hall angle to the basis and three-center terms can be related [84] to the sharp peak in the density of states (DoS) at the Fermi energy, $D\left(E_{F}\right)$, that originates in the very flat X-W-L-K $d$ band [85] whose dispersion depends sensitively on the choice of basis, Fig. 2.13. The spin-orbit splitting of the unoccupied orbitally doubly degenerate X-point state at $\sim 0.5 \mathrm{eV}$ is $0.66 \mathrm{eV}$ and of the unoccupied L-point state just above the Fermi energy is even larger at $0.93 \mathrm{eV}$ [84]. These splittings are so large that the effect of not recalculating the Fermi energy when SOC is included needs to be examined.

\section{SOC self-consistency}

So far we have determined $l_{\mathrm{sf}}$ and $\Theta_{\mathrm{sH}}$ for Pt using AS potentials calculated selfconsistently with the Stuttgart TB-LMTO code for $s p d$ and $s p d f$ bases without (w/o) SOC; in the latter, the $f$ states were included by downfolding. For the scattering calculations, these potentials were used to construct a Hamiltonian matrix including the spin-orbit interaction $H_{\mathrm{so}}$ in an LMTO basis [46] but using the Fermi energies calculated without SOC. The results obtained with an $s p d$ or $s p d f$ basis using only two center terms in $H_{\text {so }}$ are reproduced in the first row of Table 2.4 from Table $2.1\left(l_{\mathrm{sf}}\right)$ and Table $2.3\left(\Theta_{\mathrm{sH}}\right)$ and labelled "w/o SOC".

To include SOC self-consistently, we generate new spd and $s p d f$ potentials for 
Table 2.4: Dependence of the SDL $l_{\mathrm{sf}}$ and the spin Hall angle $\Theta_{\mathrm{sH}}$ for RT Pt on whether or not the Fermi energy was calculated without (w/o) or with SOC. To compare the results obtained with $s p d$ and $s p d f$ bases, only two-center terms in the SOC were included in the scattering calculations. A $5 \times 5$ supercell was used with a k-point sampling equivalent to $160 \times 160$ for a $1 \times 1$ supercell.

\begin{tabular}{ccccc}
\hline \hline & \multicolumn{2}{c}{$l_{\text {sf }}(\mathrm{nm})$} & \multicolumn{2}{c}{$\Theta_{\mathrm{sH}}(\%)$} \\
\cline { 2 - 5 } & $s p d$ & $s p d f$ & $s p d$ & $s p d f$ \\
\hline w/o SOC & $5.65 \pm 0.08$ & $5.21 \pm 0.07$ & $3.79 \pm 0.06$ & $2.95 \pm 0.03$ \\
with SOC & $5.28 \pm 0.09$ & $5.30 \pm 0.09$ & $4.27 \pm 0.03$ & $3.16 \pm 0.02$ \\
\hline \hline
\end{tabular}

Pt as input for scattering calculations using a version of the Stuttgart LMTO-ASA code extended to include SOC self-consistently ${ }^{\top}$. The results obtained with these potentials, labelled "with SOC" are shown in the second row of Table 2.4. The change found in Sec. 2.3.1 for $l_{\mathrm{sf}}^{\mathrm{Pt}}$ on going from an $s p d$ to an $s p d f$ basis is almost completely eliminated for the self-consistent SOC potentials to yield a best estimate of $l_{\mathrm{sf}}^{\mathrm{Pt}}=5.3 \pm 0.4 \mathrm{~nm}$.

For $\Theta_{\mathrm{sH}}$, the discrepancy between values found with spd and $s p d f$ bases remains. With an $s p d f$ basis we find $\Theta_{\mathrm{sH}}=3.16 \pm 0.02 \%$. Including a correction for three center terms of $5.0-3.7 \%$ from Table 2.3, our best estimate for $\Theta_{\mathrm{sH}}^{\mathrm{Pt}}$ is $3.2+5.0-3.7=4.5 \%$ with an uncertainty of about one percent.

\subsection{Comparison with other work}

\section{Experiment}

A 2007 review [10] of spin-diffusion lengths in metals and alloys contains a single entry for Pt (also for $\mathrm{Nb}, \mathrm{Pd}, \mathrm{Ru}$, and $\mathrm{W}$ ) and just a handful for Py. The entry for Pt refers to measurements at low temperatures necessitated by the use of superconducting leads in conjunction with spin-valves (SV) in a CPP geometry [86]. These $\mathrm{SV}$ measurements were interpreted within the framework of diffusive transport and led to an estimate of $l_{\mathrm{sf}}^{\mathrm{Pt}} \sim 14 \pm 6 \mathrm{~nm}$ but without a clear picture as to the microscopic origin of the diffusive scattering at the liquid He measurement temperatures. A common refrain in this section will be the need for detailed characterization of samples relating their transport properties to their microscopic structures and composition in order to make further progress.

\footnotetext{
"We use Mark van Schilfgaarde's "lm" extension of the Stuttgart LMTO code that treats noncollinear magnetization and spin-orbit coupling and is maintained in the "QUESTAAL" suite at https://www.questaal.org.
} 
Pt: $l_{\mathrm{sf}}$ and $\Theta_{\mathrm{sH}}$

At about the same time, the first electrical measurement of an ISHE was reported for the light metal $\mathrm{Al}$ [87]. Although the nonlocal measurement technique used was not directly applicable to heavy metals like Pt with short SDLs [86], it did herald the development of a number of new methods that were potentially suitable [11, 12]. The first spin-pumping (SP-ISHE) [22, 23, 28, 88], nonlocal spin-absorption (NLSA) [21, 89], spin-transfer torque FMR (SHE-STT-FMR) [24] measurements established the feasibility of measuring the I(SHE) for materials like Pt but quantitative estimates of $\Theta_{\mathrm{sH}}$ required knowledge of $l_{\mathrm{sf}}$; extensive use was made of the only value available at the time from the low temperature CPP-SV measurements [86]. Some ten years later, a review contained 22 room temperature entries for Pt [12] with $l_{\mathrm{sf}}$ ranging from $1.2 \pm 0.1$ to $11 \pm 2 \mathrm{~nm}$ and $\Theta_{\mathrm{sH}}$ from 0.37 to $12 \pm 4 \%$. We briefly discuss (some of) these experimental determinations in order to identify what needs to be done to improve the confrontation of theory and experiment.

Even for groups performing the same measurements, large differences emerged. Mosendz et al. [23] reported $\Theta_{\mathrm{sH}}=1.3 \pm 0.2 \%$ using a value of $l_{\mathrm{sf}}=10 \pm 2 \mathrm{~nm}$ they (incorrectly?) attributed to Kurt et al. [86] Performing essentially the same SP-ISHE measurements, Azevedo et al. [90] were able to determine a value of $l_{\mathrm{sf}}=3.7 \pm 0.2 \mathrm{~nm}$ by varying the thickness of $\mathrm{Pt}$ that then yielded an estimate for $\Theta_{\mathrm{sH}}=8 \pm 1 \%$. However, they used as input the Pt conductivity measured by Mosendz et al. [22] though such properties are very sensitive to where and how samples are prepared. Because of such sample to sample variability, it is very desirable to measure as many properties as possible on the same samples.

In the work cited in Table 2.5, both $l_{\mathrm{sf}}$ and $\Theta_{\mathrm{sH}}$ were extracted from measurements on the same samples, usually by varying the thickness of the Pt layer. In the experimental results shown in the top half of the table, no attempt was made to take the interface properties of the $\mathrm{FM} \mid \mathrm{Pt}$ or $\mathrm{NM} \mid \mathrm{Pt}$ interfaces into account and we see that $l_{\mathrm{sf}}^{\mathrm{Pt}}$ ranges between 1.2 and $8.3 \mathrm{~nm}$, while $\Theta_{\mathrm{sH}}$ lies in the range 1-11\%. The realization that interfaces play an essential role in degrading spin currents $[15,29,30]$ and that $l_{\text {sf }}$ might be correlated with the enhancement of thin film resistivities by interface and surface scattering [36] seemed to offer the possibility to resolve the difficulty posed by the spread in $l_{\mathrm{sf}}$ and $\Theta_{\mathrm{sH}}$ values.

However, if we look at the work cited in the bottom half of Table 2.5 that attempted to take interface SML or transparency into account, the situation has if anything worsened. We see that $l_{\mathrm{sf}}^{\mathrm{Pt}}$ ranges from 1.4 to $11 \mathrm{~nm}$ and find values for $\Theta_{\mathrm{sH}}$ as low as 3\% and as high as 39\%. Whereas the RT resistivity of bulk crystalline Pt is known to be $10.8 \mu \Omega \mathrm{cm}$ [73], we see a wide range of resistivities, from 20 to $48 \mu \Omega \mathrm{cm}$. 


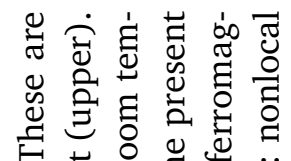

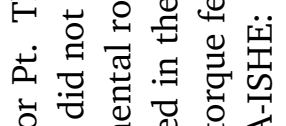

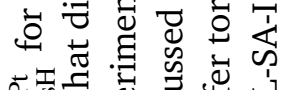

(1)

范 范

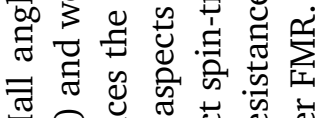

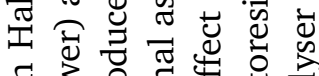

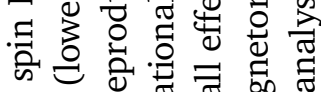

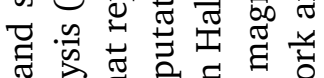

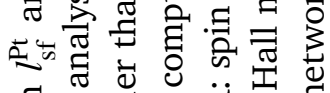

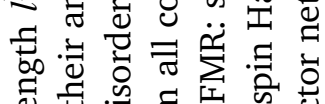

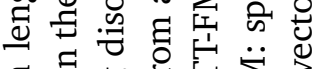

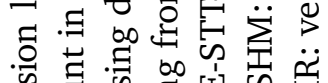

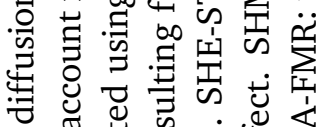

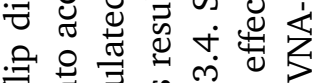

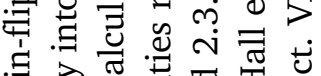

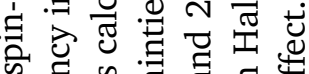

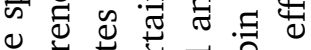

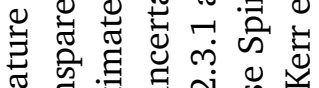

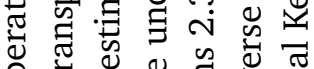

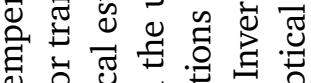

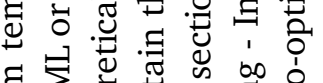

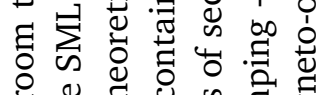

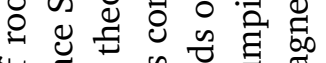

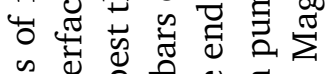

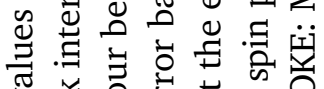

गु 可

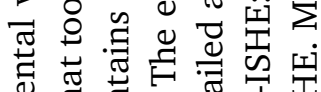

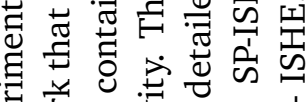

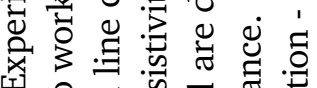

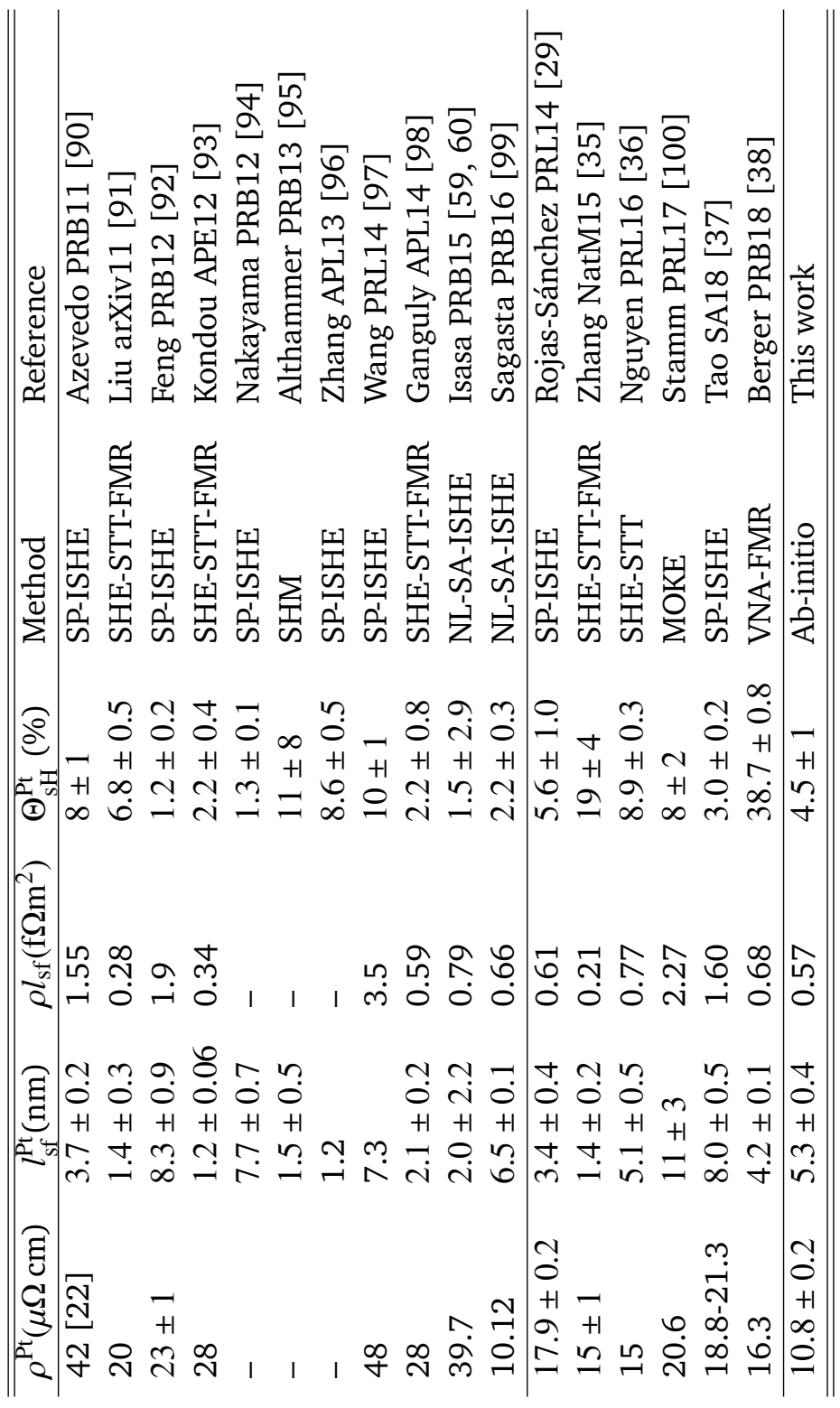

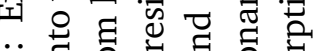

ن่ ن

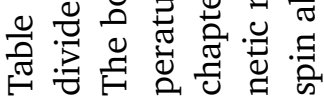


Because it has long been known that the scattering from surfaces and interfaces in thin films leads to enhanced resistivity this is not very surprising. However, little is known about the microscopic nature of the corresponding disorder on an atomic scale making it difficult to predict how it might affect the SDL and spin Hall effect. The product $\rho l_{\mathrm{sf}}$ is seen to span a much larger range between 0.21 and 2.27 $\mathrm{f} \Omega \mathrm{m}^{2}$. In view of the values of $l_{\mathrm{sf}}^{\mathrm{Pt}}$ and $\Theta_{\mathrm{sH}}$ that we calculate for bulk Pt, we can only conclude that many experiments are not at present probing the corresponding phenomena in bulk materials but are dominated by extrinsic effects - a situation very reminiscent of the discussion relating to the polarization of ferromagnets until the current-induced spin wave Doppler measurement technique was developed capable of probing the polarization far from surfaces and interfaces [57].

\section{Py: $l_{\text {sf }}$ and $\beta$}

Though it is used in a wide range of experiments and much is known about its magnetic properties, relatively few studies have been made of the transport parameters of bulk Permalloy at room temperature. These are compiled in Table 2.6 together with our best RT estimates of $l_{\mathrm{sf}}^{\mathrm{Py}}=2.8 \mathrm{~nm}$ and $\beta=0.75$. With the exception of Kimura's 2008 value [101] and in spite of the reported resistivities being much higher than the bulk value of $\rho_{\mathrm{Py}}=15.4 \mu \Omega \mathrm{cm}$ [81], there is excellent agreement between values of $l_{\mathrm{sf}}^{\mathrm{Py}}$ extracted from various experiments [39, 102, 103] and our best theoretical estimte.

Table 2.6: Experimental values of room temperature spin-flip diffusion length $l_{\mathrm{sf}}^{\mathrm{Py}}$ and spin polarization $\beta$ for Py compared with our best estimated values values calculated with lattice and spin disorder that reproduce the experimental room temperature resistivity and magnetization. The error bars contain the uncertainties resulting from all computational aspects discussed in the present chapter and are detailed at the ends of sections 2.3.2 and 2.3.3. NLSV: nonlocal spin valve. SSEISHE: spin Seebeck effect + inverse spin Hall effect. SA-LSV: spin absorption in lateral spin valves. SW-DS: spin wave Doppler shift.

\begin{tabular}{cllll}
\hline \hline$\rho(\mu \Omega \mathrm{cm})$ & \multicolumn{1}{c}{$l_{\mathrm{sf}}^{\mathrm{Py}}(\mathrm{nm})$} & \multicolumn{1}{c}{$\beta$} & Method & \multicolumn{1}{c}{ Ref. } \\
\hline 26.8 & 3 & 0.25 & NLSV & Kimura PRB05 [102] \\
23.1 & 4.5 & 0.49 & NLSV & Kimura PRL08 [101] \\
$\sim 30$ & 2.5 & - & SSE-ISHE & Miao PRL13 [39] \\
44 & $2.30 \pm 0.61$ & $0.31 \pm 0.02$ & SA-LSV & Sagasta APL17 [103] \\
$29 \pm 3$ & - & $0.61 \pm 0.02$ & SW-DS & Zhu PRB10 [104] \\
25 & - & 0.71 & SW-DS & Haidar PRB13 [58] \\
\hline $15.4 \pm 0.2$ & $2.8 \pm 0.1$ & $0.75 \pm 0.01$ & Ab-initio & This work \\
\hline \hline
\end{tabular}

The polarizations reported from the non-local spin valve experiments [101- 
103] are however much smaller than our bulk value, $\beta=0.75 \pm 0.01$. Two studies $[58,104]$ measured $\beta$ independent of $l_{\mathrm{sf}}$ using spin-wave Doppler shift experiments. Haidar and Bailleul [58] carried out systematic thickness dependent measurements at room temperature and predicted an extrapolated bulk value of 0.71 . In the nonlocal spin valve based spin absorption experiments where $l_{\mathrm{sf}}^{\mathrm{Py}}$ and $\beta$ were determined simultaneously, the assumption of transparent $\mathrm{Py} \mid \mathrm{Cu}$ interfaces may have affected the determination of $\beta$ [101-103]. Alternatively, with Fig. 2.9 in mind, it is tempting to speculate that these experiments are probing an interface property rather than a property of bulk Py.

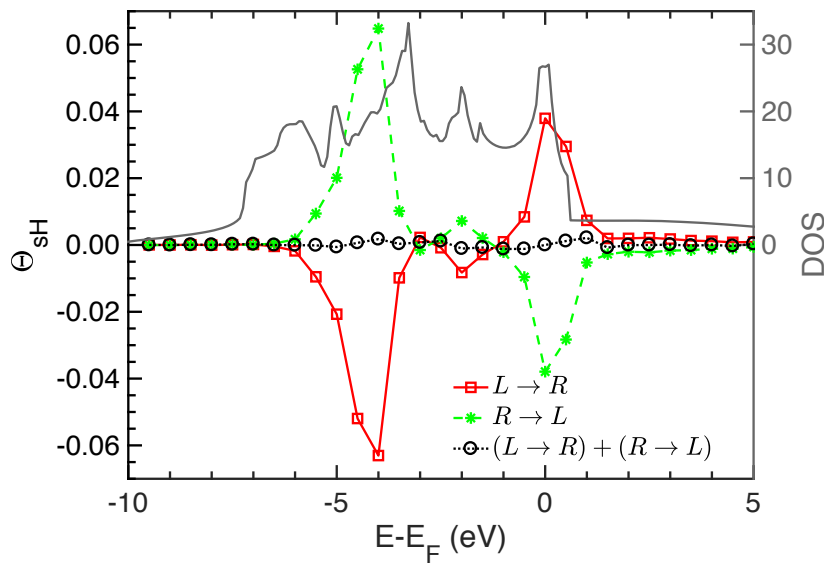

Figure 2.14: Spin Hall angle as a function of the energy in a rigid band approximation calculated for electrons incident from the left (red squares) and from the right (green stars). The sum of these contributions is shown as black circles. For reference, we show the Pt $d$-density of states in grey.

\section{Other calculations}

We are not aware of any theoretical studies of $l_{\mathrm{sf}}$ in either Pt or Py. There have been a number of studies of the "intrinsic" spin Hall conductivity (SHC) of bulk Pt that only depends on the electronic band structure of the crystalline material and can be evaluated in linear response by taking the $\omega \rightarrow 0$ limit of the optical conductivity using electronic structures calculated from first principles [84] or tight binding fits to first principles band structures [105]. In materials with strong spinorbit coupled bands, it would appear that the intrinsic contribution dominates the SHC. The largest contributions arise from orbital degeneracies close to the Fermi energy. The inclusion of finite temperatures for the electrons via the Fermi Dirac function leads to a rapid quenching of the SHC in this picture [84].

Our calculation of the SHA is also "intrinsic" in the sense that no impurities are 
involved but as shown by Wang et al. it also leads to a different temperature dependence. In Ref. [32] we found that the SHA is essentially linear in temperature so that the SHC is temperature independent. In Fig. 2.14, we show how the SHA depends on the band filling. Like Guo we identify two prominent peaks that arise when the Fermi level coincides with orbital degeneracies at high symmetry points in the Brillouin zone. These features quite clearly survive lattice disorder. By calculating the contribution to the SHA from electrons propagating from $L \rightarrow R$ and from $R \rightarrow L$, we can obtain the so called "Fermi sea" contribution to the SHA by direct summation. Unlike the case of charge transport where filled bands make no contribution, there is no guarantee that this will always be the case for spin transport $[62,106]$. In the absence of disorder, time reversal and inversion symmetry lead to Kramers degeneracy and the Fermi sea contribution vanishes identically. Thermal disorder breaks inversion symmetry locally and lifts the Kramers degeneracy. In the present case however, the resulting contribution is entirely negligible.

\subsection{Summary and Conclusions}

We have developed a method to calculate localized charge and spin currents in a multilayer system from the results of first-principles scattering calculations that include thermal lattice and spin disorder as well as chemical disorder for alloys. This allows us to factor out the effect of the interfaces that are unavoidable in scattering calculations and quantitatively evaluate parameters for bulk materials of interest in spin transport studies. We illustrated it by calculating the spin-flip diffusion length for Py $l_{\mathrm{sf}}^{\mathrm{Py}}=2.8 \pm 0.1 \mathrm{~nm}$ and Pt $l_{\mathrm{sf}}^{\mathrm{Pt}}=5.3 \pm 0.4 \mathrm{~nm}$, the bulk spin polarization $\beta=0.75 \pm 0.01$ for Py and the spin Hall angle $\Theta_{\mathrm{sH}}=4.5 \pm 1.0 \%$ for Pt at room temperature. Here the uncertainties were identified by systematically examining the approximations that must necessarily be made in calculations with finite computational resources.

A comparison of the calculated bulk transport parameters with experimental results was inconclusive because, we believe, experiment is not able to unambiguously identify the bulk transport regime in the case of $l_{\mathrm{sf}}$ and $\Theta_{\mathrm{sH}}$ for Pt and many reported results are dominated by interface effects. Although recent attempts have been made to incorporate interface effects into the interpretation of experiments for bilayers, the effect of doing so appears to lead to diverging results rather than convergence [29, 35-38].

The study presented in this chapter opens up a wide range of possibilities to predict systematic trends for material parameters essential for spintronics applications. One possibility is to extend the calculations presented here to determine $l_{\mathrm{sf}}$ and $\beta$ for other bulk magnetic systems; to determine $\Theta_{\mathrm{sH}}$ and $l_{\mathrm{sf}}$ for other bulk $5 d, 4 d$ and $3 d$ metals and their alloys all as a function of temperature with a view to identifying suitable candidates for spintronics applications and to better understand 
their temperature dependence and underlying scattering mechanisms. Another very promising direction is to use the localized spin currents to focus on interface effects and help disentangle bulk and interface contributions in the experimental studies we discussed briefly in the previous section. The latter direction will be pursued in Chapter 3 and Chapter 4. 


\section{3 \\ Calculating interface transport parameters at finite temperatures: Nonmagnetic interfaces*}

We use first-principles scattering calculations to investigate spin transport through interfaces between nonmagnetic metals where the symmetry-lowering leads to an enhancement of the effect of spin-orbit coupling (SOC) and to a discontinuity of the spin currents passing through the interfaces. By calculating the conductance and local spin currents for nonmagnetic bilayers, we extract values of the room temperature spin memory loss $\delta$ and of the interface spin Hall angle $\Theta_{\mathrm{I}}$ along with the interface resistance $R_{\mathrm{I}}$ for nonmagnetic $A u \mid P t$ and $A u \mid P d$ interfaces using a frozen thermal disorder scheme to model finite temperatures. Substantial values of all three parameters are found with important consequences for experiments involving nonmagnetic spacer and capping layers. The temperature dependence of the interface parameters is determined for $\mathrm{Au} \mid \mathrm{Pt}$.

"To be submitted as: K. Gupta, Z. Yuan, P.J. Kelly, First-principles spin transport at finite temperatures: nonmagnetic interfaces 


\subsection{Introduction}

In Chapter 2, we introduced a formalism to evaluate localized charge and spin currents from the solutions of fully relativistic quantum mechanical scattering calculations. We obtained a layer-resolved description of spin currents propagating and diffusing through atomic layers of thermally disordered Pt and Py. This allowed us to distinguish bulk from interface behaviour in these materials. Specifically for $\mathrm{Pt}$, we observed a large deviation from bulk behaviour close to the interface with a lead. Although an interface between a room temperature diffusive Pt slab and a zero temperature ballistic lead would be very difficult to realize experimentally, the deviation we observed pointed towards a strong interface spin-orbit splitting, which is not unexpected considering the strength of the spin-orbit coupling (SOC) in Pt. This has led us to use the local spin current scheme in this chapter to study what happens at realistic interfaces between two materials, both at room temperature.

Even though it is generally recognized that interfaces play an extremely important role in influencing spin transport through magnetic multilayers, it has turned out to be very difficult to take this into account in interpreting experiments because of the additional interface parameters that this would involve; spin transport experiments already involve a large number of poorly known bulk parameters like the spin-flip diffusion length (SDL) and the conductivity polarization. As an example, we consider spin pumping-inverse spin Hall effect (SHE) measurements $[23,28,90]$ for ferromagnetic|nonmagnetic (FM|NM) bilayers. The spin current pumped into the NM material by ferromagnetic resonance-induced magnetization precession leads to an interface enhancement of the Gilbert magnetization damping [26, 27, 107]. Experiments [29] and first-principles calculations [30] have shown that the spin current lost at the interface, expressed in terms of a "spin memory loss" parameter $\delta$, must be incorporated in the damping enhancement in order to correctly estimate the bulk spin-flip diffusion length $l_{\mathrm{NM}} \equiv l_{\mathrm{sf}}^{\mathrm{NM}}$ and bulk spin Hall angle (SHA) $\Theta_{\mathrm{NM}} \equiv \Theta_{\mathrm{sH}}^{\mathrm{NM}}$ in the NM metal. The pumped spin current can also give rise to an interface SHE parameterized in terms of an additional parameter, the interface SHA [32]. If the NM metal has an interface with a seeding or a capping layer, additional interface effects might need to be considered. Analysis of experiments with a view to extracting the bulk parameters $l_{\mathrm{NM}}$ and $\Theta_{\mathrm{NM}}$ needs to include the possibility of not only the interface effects just mentioned [37] but also the magnetic proximity effect (MPE) that renders a few layers of NM metal adjacent to the FM magnetic; this has also been claimed to modify the damping enhancement [108].

In such a scenario, it is crucial to have a way of determining the interface parameters independently. The spin memory loss parameter $\delta$ is typically extracted from current-perpendicular-to-plane (CPP) magnetoresistance experiments [10, 17] using the semiclassical Valet-Fert (VF) model [9]. While these experiments are relatively simple to interpret, they are restricted to low temperatures as they require 
superconducting leads. In this chapter, we will use the VF model to extract $\delta$ and the interface SHA for $\mathrm{NM} \mid \mathrm{NM}^{\prime}$ interfaces from spin currents. These interfaces are free of any magnetization that could influence interface parameters by way of an MPE or simply through the exchange interaction. We can thus focus on the role of interface spin-orbit splitting for the heavy transition metals Pt and Pd. FM|NM interfaces will be addressed in the following chapter.

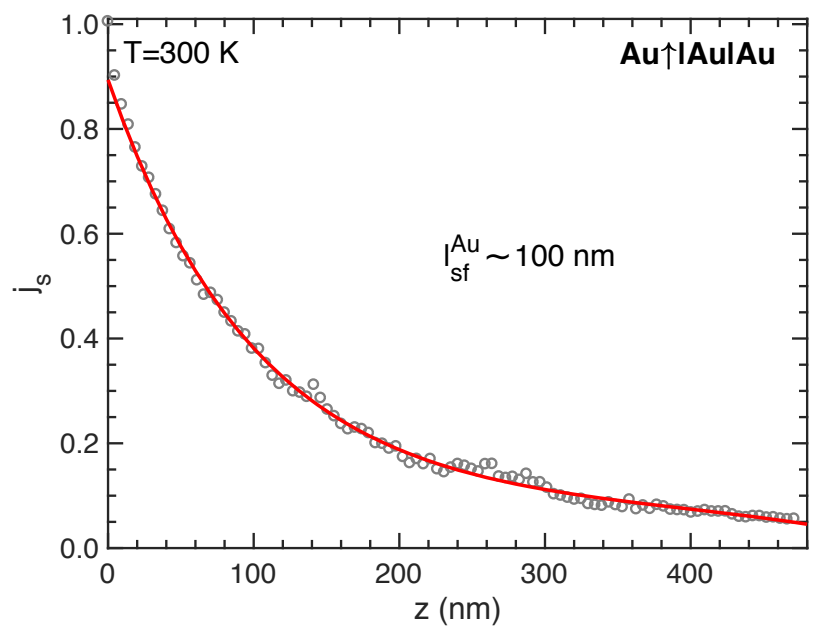

Figure 3.1: A fully polarized spin current $\left(j_{s}(0)=1\right)$ is injected into $470 \mathrm{~nm}$ of diffusive $\mathrm{Au}$ at $300 \mathrm{~K}$ for a $4 \times 4$ lateral supercell. The open circles indicate the calculated spin current profile. An exponential fit yields $l_{\mathrm{Au}} \sim 100 \mathrm{~nm}$.

The $\mathrm{NM} \mid \mathrm{NM}^{\prime}$ interfaces we will construct are $\mathrm{Au} \mid \mathrm{Pt}$ and $\mathrm{Au} \mid \mathrm{Pd}$. This choice offers several advantages. Au has a low effective SOC because its $d$ and $p$ bands are completely filled or empty and only affect transport via hybridization with the free electron like $s$ band that is Kramers degenerate for an inversion symmetric material. This is expected to lead to a very long spin-flip diffusion length and small spin Hall angle. At room temperature, literature values of $l_{\mathrm{Au}}$ are reported in the range 27$86 \mathrm{~nm}$ [12]; $\Theta_{\mathrm{Au}}$ is found to be as small as $0.05 \%$ [59] or as large as $11.3 \%$ [12, 109] and apparently depends strongly on the thickness of the samples used in the measurements [109-111]. We can estimate $l_{\mathrm{Au}}$ at room temperature by injecting a fully polarized spin current from a "half-metallic ferromagnetic" Au lead (Au个) into a $470 \mathrm{~nm}$ thick slab of diffusive Au. The results obtained for a single configuration of thermal disorder and a small $4 \times 4$ lateral supercell are plotted in Fig. 3.1. By fitting the exponentially decaying spin current, that does not fully decrease to zero, we obtain an estimate of $l_{\mathrm{Au}} \sim 100 \mathrm{~nm}^{\dagger}$. In order to determine the interface spin

\footnotetext{
The value is only a rough estimate. Making it more quantitative would require a systematic study of how the resistivity and spin-flip diffusion length depend on supercell size, sampling of the Brillouin
} 
memory loss, we will use this construction to inject a fully polarized spin current into a thin slab of diffusive Au that will undergo minimal decay before encountering an interface with either Pt or Pd. The lattice mismatch between Au, Pt and Pd is small enough to construct pseudomorphic interfaces by compressing Au uniformly to match the other two lattices without drastically changing the electronic structure of $\mathrm{Au}$ at the Fermi energy.

Besides evaluating $\delta$, we will look to see if there is an interface spin Hall effect at the $\mathrm{Au} \mid \mathrm{Pt}$ and $\mathrm{Au} \mid \mathrm{Pd}$ interfaces. Since the prediction of Wang et al. of such an effect for Py|Pt [32], there have been experiments [112] and theoretical studies [113] that point towards a role for interface spin-orbit coupling in generating spin currents at NM|NM' interfaces. Since everything we know about nonmagnetic interfaces is through low temperature magnetoresistance experiments $[17,114]$ or through calculations for ballistic interfaces $[115,116]$, we will also investigate the temperature dependence of interface transport parameters.

The plan of the chapter is as follows. In Sec. 3.2 we first summarize the original VF model (Sec. 3.2.1) and then extend it to include the effect of SOC at interfaces and discuss how it will be used to extract interface parameters (Sec. 3.2.2). We describe how transverse spin currents generated by the spin Hall effect behave at interfaces (Sec. 3.2.3) before elaborating on the scheme we use to extract quantitative estimates of the interface SHA from inverse spin-Hall effect calculations for interfaces (Sec. 3.2.4). In Sec. 3.3 we briefly summarize the first-principles scattering theory $[45,46,117]$, give details of how $\mathrm{Au} \mid \mathrm{Pt}$ and $\mathrm{Au} \mid \mathrm{Pd}$ interface geometries are constructed and describe how temperature is incorporated in the adiabatic approximation. In Sec. 3.4, we demonstrate the procedure described in Sec. 3.2 by extracting the interface parameters for AulPt at RT (Sections 3.4.1, 3.4.2 and 3.4.4), compare them with interface parameters for AulPd (Sec. 3.4.5) and determine $\mathrm{Aul}$ Pt parameters at 200 and $400 \mathrm{~K}$ (Sec. 3.4.6). In Sec. 3.5 we summarize our findings and present some conclusions.

\subsection{Methods}

\subsubsection{Valet-Fert model}

In this subsection, we recapitulate the VF model [9] for spin transport before generalizing it to include spin-flip scattering at interfaces [16]. The macroscopic equations derived by VF characterize transport in terms of material specific parameters. For a current flowing along the $z$ direction perpendicular to the interface plane in an axially symmetric CPP geometry, the spatial profiles of majority (minority) spin-

zone, configuration averaging etc. 
polarized current densities $j_{\uparrow(\downarrow)}$ and spin accumulation $\mu_{\uparrow(\downarrow)}$ are related as follows

$$
\begin{aligned}
\frac{\partial^{2} \mu_{s}}{\partial z^{2}} & =\frac{\mu_{s}}{l^{2}}, \\
j_{\uparrow(\downarrow)}(z) & =-\frac{1}{e \rho_{\uparrow(\downarrow)}} \frac{\partial \mu_{\uparrow(\downarrow)}}{\partial z} .
\end{aligned}
$$

Here, $\mu_{s}=\mu_{\uparrow}-\mu_{\downarrow}$ is the spin accumulation, $l \equiv l_{\text {sf }}$ denotes the spin-flip diffusion length (SDL), and $\rho_{\uparrow(\downarrow)}$ is the spin-dependent bulk resistivity. According to the two-current series resistor model [9], resistances are first calculated separately for spin-up and spin-down electrons and then added in parallel. For non-magnetic materials, $\rho_{\uparrow(\downarrow)}=2 \rho$, where $\rho$ is the total resistivity. Thus, spin transport in the bulk of a material can be characterized in terms of its resistivity $\rho$ and spin-flip diffusion length $l$. Equations (3.1) and (3.2) can be solved for $\mu_{\uparrow}, \mu_{\downarrow}, j_{\uparrow}, j_{\downarrow}$ making use of the condition that the total current density $j=j_{\uparrow}+j_{\downarrow}$ is conserved in onedimensional transport. The general solution of (3.1) is $\mu_{s}(z)=A e^{z / l}+B e^{-z / l}$. The normalized spin-current density $j_{s}(z) \equiv\left[j_{\uparrow}(z)-j_{\downarrow}(z)\right] / j$ is given by

$$
j_{s}(z)=\frac{1}{2 e j \rho l}\left[B e^{-z / l}-A e^{z / l}\right]
$$

where the coefficients $A$ and $B$ can be determined by using suitable boundary conditions. Spin-dependent scattering at an interface is characterized in terms of the interface resistances $R_{\uparrow(\downarrow)}$. By analogy with the bulk resistivity, $R_{\uparrow(\downarrow)}=2 R_{\mathrm{I}}$ where $R_{\mathrm{I}}$ is the total interface resistance.

\subsubsection{Interface discontinuity}

The model described above was extended by Fert and Lee [118] to include the effect of interface SOC in terms of additional spin-flip interface resistances. The effect of interface spin flipping was first described in terms of the spin-memory loss parameter $\delta$ by Baxter et al. for $\mathrm{NM} \mid \mathrm{NM}^{\prime}$ interfaces between two nonmagnetic metals [16]. In this subsection, we summarize this generalized VF model and extract the boundary conditions for a geometrically sharp $\mathrm{NM} \mid \mathrm{NM}^{\prime}$ interface.

In Fig. 3.2 we sketch how a spin current passes through a nonmagnetic bilayer. A fully polarized spin current $j_{s}(0)=1$ enters the first nonmagnetic layer, $\mathrm{NM}_{1}$, from the left. The SDL in this material, $l_{1}$, determines the spatial decay of $j_{s}(z)$. For a slab length corresponding to a multiple $N$ of $l_{1}, j_{s}\left(N l_{1}\right)=e^{-N} \sim(0.37)^{N}$ at the right boundary of $\mathrm{NM}_{1}$. An interface breaks inversion symmetry and lifts the Kramers degeneracy. SOC-induced energy band splittings are enhanced by the symmetry lowering so that the spin current decays rapidly in the vicinity of the interface leading to an interface discontinuity in $j_{s}(z)$. In the semiclassical framework, it is quantified in terms of the spin memory loss parameter $\delta$. After the abrupt decay 


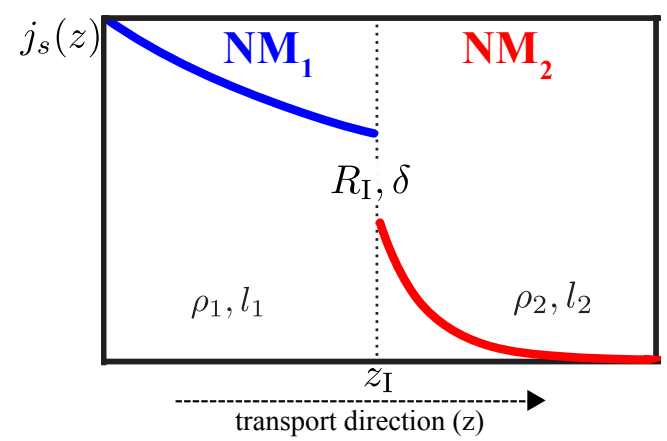

Figure 3.2: Valet-Fert model for spin-flip scattering in a nonmagnetic bilayer. A fully polarized spin current is injected into $\mathrm{NM}_{1}$ from a ballistic half-metallic lead. The decay of current in $\mathrm{NM}_{i}$ is parametrized by the resistivity $\rho_{i}$ and spin-flip diffusion length $l_{i}$ of material $i=1,2$. Because of the enhancment of the effective spin-orbit interaction at an interface, the spin current is discontinuous at $z_{\mathrm{I}}$ (position of the geometrical interface). This discontinuity is characterized in terms of the interface resistance $R_{\mathrm{I}}$ and spin-memory loss $\delta$.

at the interface, the spin current that survives in $\mathrm{NM}_{2}$ decays to zero on a length scale described by the SDL $l_{2}$.

By fitting $j_{s}(z)$ calculated quantum mechanically from scattering theory to the corresponding VF equation, we obtain values of $j_{s}\left(z_{\mathrm{I}}\right)$ on either side of the interface. To extract $\delta$, the interface discontinuity is incorporated into the VF framework. It is assumed that the interface region (I) has a finite thickness $t$ and can be treated as a material with resistivity $\rho_{\mathrm{I}}$ and spin-flip diffusion length $l_{\mathrm{I}}$. In terms of these "bulk" parameters, the areal interface resistance $A R_{\mathrm{I}}$ and spin-flip diffusion length are

$$
A R_{\mathrm{I}}=\lim _{t \rightarrow 0} \rho_{\mathrm{I}} t \quad \text { and } \quad \delta=\lim _{t \rightarrow 0} t / l_{\mathrm{I}}
$$

With the above description of the interface, a bilayer of any two non-magnetic bulk materials $\left(\mathrm{NM}_{1}\right.$ and $\left.\mathrm{NM}_{2}\right)$ becomes a fictitious trilayer $\mathrm{NM}_{1}|\mathrm{I}| \mathrm{NM}_{2}$. Spin transport in this geometry can thus be characterized by six bulk transport parameters $\rho_{1}, \rho_{\mathrm{I}}, \rho_{2}, l_{1}, l_{\mathrm{I}}, l_{2}$ instead of $\rho_{1}, \rho_{2}, A R_{\mathrm{I}}, l_{1}, l_{2}$ and $\delta$. The generalized spin transport equations for the three distinct layers labelled $i=1,2$ and I are

$$
\begin{aligned}
\mu_{s i}(z) & =A_{i} e^{z / l_{i}}+B_{i} e^{-z / l_{i}} \\
j_{s i}(z) & =\frac{1}{2 e j \rho_{i} l_{i}}\left[B_{i} e^{-z / l_{i}}-A_{i} e^{z / l_{i}}\right] .
\end{aligned}
$$

To switch from an $\mathrm{NM}_{1} \mid \mathrm{NM}_{2}$ to an $\mathrm{NM}_{1}|\mathrm{I}| \mathrm{NM}_{2}$ description, the discontinuity at the sharp interface at $z=z_{\mathrm{I}}$ in $\mu_{s}\left(z_{\mathrm{I}}\right)$ and $j_{s}\left(z_{\mathrm{I}}\right)$ becomes a continuous transition through the interface layer between $z=z_{\mathrm{I}}$ and $z=z_{\mathrm{I}}+t$ (Fig. 3.3). Continuity of 


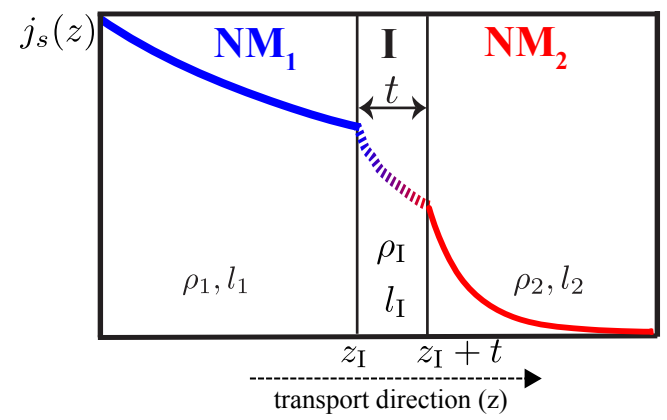

Figure 3.3: Generalized VF model for a $\mathrm{NM}_{1}|\mathbf{I}| \mathrm{NM}_{2}$ trilayer. The interface is modelled as a fictitious bulk-like layer with thickness $t$ that is characterized by parameters $\rho_{\mathrm{I}}$ and $l_{\mathrm{I}}$, such that $\rho_{\mathrm{I}}=A R_{\mathrm{I}} / t$ and $l_{\mathrm{I}}=t / \delta$. Ths spin current is continuous at the $\mathrm{NM}_{1} \mid \mathrm{I}$ and $\mathrm{I} \mid \mathrm{NM}_{2}$ interfaces.

$\mu(z)$ at the $\mathrm{NM}_{1} \mid \mathrm{I}$ and $\mathrm{I} \mid \mathrm{NM}_{2}$ interfaces yields the equations

$$
\begin{aligned}
\mu_{s 1}\left(z_{\mathrm{I}}\right) & =A_{\mathrm{I}} e^{z_{\mathrm{I}} / l_{\mathrm{I}}}+B_{\mathrm{I}} e^{-z_{\mathrm{I}} / l_{\mathrm{I}}}, \\
\mu_{s 2}\left(z_{\mathrm{I}}+t\right) & =A_{\mathrm{I}} e^{z_{\mathrm{I}} / l_{\mathrm{I}}} e^{\delta}+B_{\mathrm{I}} e^{-z_{\mathrm{I}} / l_{\mathrm{I}}} e^{-\delta},
\end{aligned}
$$

and of $j_{s}(z)$

$$
\begin{aligned}
j_{s 1}\left(z_{\mathrm{I}}\right) & =\frac{1}{2 e j \rho_{\mathrm{I}} l_{\mathrm{I}}}\left[B_{\mathrm{I}} e^{-z_{\mathrm{I}} / l_{\mathrm{I}}}-A_{\mathrm{I}} e^{z_{\mathrm{I}} / l_{\mathrm{I}}}\right], \\
j_{s 2}\left(z_{\mathrm{I}}+t\right) & =\frac{1}{2 e j \rho_{\mathrm{I}} l_{\mathrm{I}}}\left[B_{\mathrm{I}} e^{-z_{\mathrm{I}} / l_{\mathrm{I}}} e^{-\delta}-A_{\mathrm{I}} e^{z_{\mathrm{I}} / l_{\mathrm{I}}} e^{\delta}\right] .
\end{aligned}
$$

Eliminating $A_{\mathrm{I}}$ and $B_{\mathrm{I}}$ and taking the limit $t \rightarrow 0$ leads to the expected discontinuity in $\mu_{s}$ and $j_{s}$ at the $\mathrm{NM}_{1} \mid \mathrm{NM}_{2}$ interface. Substituting (3.4) then yields

$$
\begin{aligned}
& j_{s 1}\left(z_{\mathrm{I}}\right)=\frac{\delta}{2 e j A R_{\mathrm{I}} \sinh \delta}\left[\mu_{s 1}\left(z_{\mathrm{I}}\right) \cosh \delta-\mu_{s 2}\left(z_{\mathrm{I}}\right)\right], \\
& j_{s 2}\left(z_{\mathrm{I}}\right)=\frac{\delta}{2 e j A R_{\mathrm{I}} \sinh \delta}\left[\mu_{s 1}\left(z_{\mathrm{I}}\right)-\mu_{s 2}\left(z_{\mathrm{I}}\right) \cosh \delta\right] .
\end{aligned}
$$

Use of the remaining boundary conditions: $j_{s}(0)=1, j_{s}(\infty)=0$ allows us to express $\mu_{s 1}\left(z_{\mathrm{I}}\right)$ and $\mu_{s 2}\left(z_{\mathrm{I}}\right)$ in terms of $j_{s 1}\left(z_{\mathrm{I}}\right)$ and $j_{s 2}\left(z_{\mathrm{I}}\right)$. After some algebra, we obtain

$$
\begin{aligned}
& j_{s 1}\left(z_{\mathrm{I}}\right)=\frac{\delta}{R_{\mathrm{I}} \sinh \delta}\left[\rho_{1} l_{1} \cosh \delta\left\{\operatorname{coth}\left(\frac{z_{\mathrm{I}}}{l_{1}}\right)\left[j_{s 1}\left(z_{\mathrm{I}}\right)-e^{z_{\mathrm{I}} / l_{1}}\right]+e^{z_{\mathrm{I}} / l_{1}}\right\}-\rho_{2} l_{2} j_{s 2}\left(z_{\mathrm{I}}\right)\right] \\
& j_{s 2}\left(z_{\mathrm{I}}\right)=\frac{\delta}{R_{\mathrm{I}} \sinh \delta}\left[\rho_{1} l_{1}\left\{\operatorname{coth}\left(\frac{z_{\mathrm{I}}}{l_{1}}\right)\left[j_{s 1}\left(z_{\mathrm{I}}\right)-e^{z_{\mathrm{I}} / l_{1}}\right]+e^{z_{\mathrm{I}} / l_{1}}\right\}-\rho_{2} l_{2} j_{s 2}\left(z_{\mathrm{I}}\right) \cosh \delta\right] .
\end{aligned}
$$




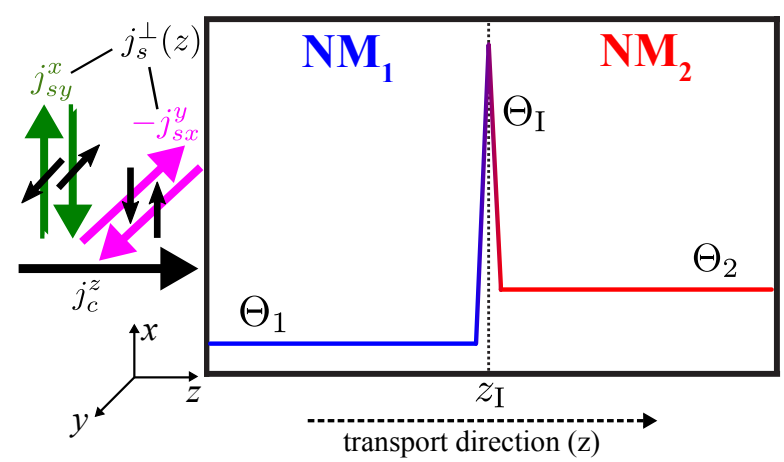

Figure 3.4: Sketch of the transverse spin currents $j_{s \alpha}^{\perp}\left(j_{s y}^{x}\right.$, vertical green arrows and $-j_{s x}^{y}$, pink arrows) generated in response to a constant charge current $j_{c}^{z}=j$ (horizontal black arrow) through a $\mathrm{NM}_{1} \mid \mathrm{NM}_{2}$ bilayer. $\Theta_{1}$ and $\Theta_{2}$ represent the ratio $j_{s \alpha}^{\perp} / j$ in $\mathrm{NM}_{1}$ and $\mathrm{NM}_{2}$ respectively. A sharp peak in $j_{s}^{\perp}$ at the interface is attributed to an interface spin Hall effect described by the angle $\Theta_{\mathrm{I}}$.

In principle we can solve either of the above two equations numerically by substituting values of all parameters and $j_{s 1}\left(z_{\mathrm{I}}\right)$ and $j_{s 2}\left(z_{\mathrm{I}}\right)$ to find $\delta$. However, as mentioned in the introduction, $l_{\mathrm{Au}}$ cannot be easily determined accurately. To avoid using $l_{\mathrm{Au}}$ in extracting $\delta$, we eliminate $\rho_{1} l_{1}$ in the above two equations to yield

$$
\frac{j_{s 1}\left(z_{\mathrm{I}}\right)}{j_{s 2}\left(z_{\mathrm{I}}\right)}=\cosh \delta+\frac{\rho_{2} l_{2}}{A R_{\mathrm{I}}} \delta \sinh \delta
$$

expressing $\delta$ in terms of $j_{s 1}\left(z_{\mathrm{I}}\right)$ and $j_{s 2}\left(z_{\mathrm{I}}\right)$ as well as $\rho_{2}, l_{2}$ and $R_{\mathrm{I}}$.

We will determine $\rho_{2}$ and $A R_{\mathrm{I}}$ from calculations of the conductance. The spinflip diffusion length $l_{2}$ will be extracted using the method described in Sec. 2.3.1 and illustrated in Fig. 3.1 for Au. $j_{s 1}\left(z_{\mathrm{I}}\right)$ and $j_{s 2}\left(z_{\mathrm{I}}\right)$ will be determined by fitting the spin current in $\mathrm{NM}_{1} \mid \mathrm{NM}_{2}$ using (3.6).

\subsubsection{Transverse spin current at an interface}

When a charge current is passed through a nonmagnetic bulk material, the SOC leads to a transverse spin current in an effect called the spin Hall effect [11, 12, 19, 119]. This spin current can be denoted $j_{s \alpha}^{\perp}$ where $\alpha$ labels the direction of the spin polarization that is given by the vector product of the driving charge current and the induced transverse spin current. As sketched in Fig. 3.4 for a constant charge current in the $z$ direction, $j \equiv j_{c}^{z}$, transverse spin currents are generated in the radial direction e.g. $x$ and $-y$ directions that are polarized in the $y$ and $x$ directions, respectively. The amount of spin current generated per unit charge current is given by the spin Hall angle $\Theta_{\mathrm{sH}}=j_{s \alpha}^{\perp} / j$. By measuring charge currents in terms of the fundamental unit of charge $-|e|$ and spin currents in units of $\hbar / 2$, $\Theta_{\mathrm{sH}}$ becomes dimensionless. 


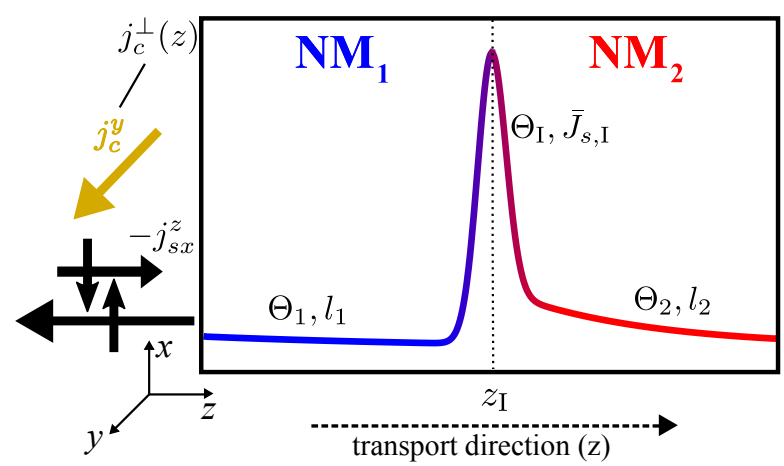

Figure 3.5: On injecting a spin polarized current, whose polarization is perpendicular to the current direction, into a $\mathrm{NM}_{1} \mid \mathrm{NM}_{2}$ bilayer, a transverse charge current $j_{c}^{\perp}$ is generated in a mutually perpendicular direction because of the inverse spin Hall effect. The spin current $j_{s x}^{z}(z)$, composed of up and down spins propagating to the left and right, respectively (black horizontal arrows), is not conserved because of SOC-induced spin flipping. It induces a transverse charge current in each layer determined by the respective spin Hall angles and results in the spatially varying $j_{c}^{\perp}(z)$ sketched in the figure. At the interface, the discontinuity in $j_{s}(z)$ that is spin memory loss (Fig. 3.2) combined with the interface spin Hall angle (Fig. 3.4) gives rise to a peak in $j_{c}^{\perp}(z)$ about $z_{\mathrm{I}}$.

When the constant charge current $j$ passes through a bilayer composed of the nonmagnetic materials $\mathrm{NM}_{1}$ and $\mathrm{NM}_{2}$ perpendicular to the $\mathrm{NM}_{1} \mid \mathrm{NM}_{2}$ interface, it gives rise to transverse spin currents $j_{s \alpha}^{\perp}$ with magnitudes given by $j \Theta_{1}$ in $\mathrm{NM}_{1}$ and $j \Theta_{2}$ in $\mathrm{NM}_{2}$, respectively; see Fig. 3.4. In the vicinity of the interface, the possibility of an abrupt deviation from the bulk behaviour resulting from an interface spin-Hall effect and described by the angle $\Theta_{\mathrm{I}}$ has been proposed [32]. Because of the finite width of this peak, it is not possible to directly extract $\Theta_{\text {I }}$ from the transverse spin currents. Instead, we will follow Wang et al. [32] and access it through the inverse spin Hall effect (ISHE) whereby a spin current polarized perpendicular to the current direction generates a transverse charge current along a mutually perpendicular direction. In the following subsection, we describe how $\Theta_{I}$ is extracted from ISHE calculations.

\subsubsection{Transverse charge current and interface SHE}

A fully polarized spin current entering an $\mathrm{NM}_{1} \mid \mathrm{NM}_{2}$ bilayer as shown in Fig. 3.2 undergoes spin-flip diffusion in each layer as described by (3.6). The spin polarization is oriented along $-x$ perpendicular to the current direction $z$. This spatially varying spin current $j_{s}(z) \equiv j_{s x}^{z}(z) / j$ induces a transverse charge current $j_{c}^{y}(z)$ in the $y$ direction. For two layers labelled $i=1,2$, the normalized charge current is given 
by $j_{c}^{y}(z)=\Theta_{i} j_{s i}(z)$ as sketched in Fig. 3.5. At the interface, the abrupt decay in spin current that is called spin memory loss, combined with an interface spin Hall angle $\Theta_{\mathrm{I}}$ yields a peak $j_{c}^{y}\left(z_{\mathrm{I}}\right)=\Theta_{\mathrm{I}} \bar{J}_{s, \mathrm{I}} \delta\left(z-z_{\mathrm{I}}\right)$. Here $\bar{J}_{s, \mathrm{I}}$ is the effective spin current at the interface. Following Wang et al. [32], we integrate the spin current and the transverse charge current in $\mathrm{NM}_{2}$ from the interface at $z_{\mathrm{I}}=0$ out to a distance $L$. As a function of $L$, the total spin current is

$$
\begin{aligned}
\bar{J}_{s}(L) & =\int_{0}^{L} \bar{J}_{s, \mathrm{I}} \delta(z)+B_{2} e^{-z / l_{2}} d z \\
& =\bar{J}_{s, \mathrm{I}}+B_{2} l_{2}\left(1-e^{-L / l_{2}}\right) .
\end{aligned}
$$

The total transverse charge current induced by the spin current is

$$
\bar{J}_{c}(L)=\Theta_{\mathrm{I}} \bar{J}_{s, \mathrm{I}}+\Theta_{2} B_{2} l_{2}\left(1-e^{-L / l_{2}}\right) .
$$

To extract $\Theta_{\mathrm{I}}$, (3.13) and (3.14) are used to fit the calculated spin and charge currents integrated out to a distance $L$. An effective spin Hall angle due to the inverse spin Hall effect can be defined as

$$
\Theta_{\mathrm{eff}}(L) \equiv \frac{\bar{J}_{c}(L)}{\bar{J}_{s}(L)}=\frac{\Theta_{\mathrm{I}} \bar{J}_{s, \mathrm{I}}+\Theta_{2} B_{2} l_{2}\left(1-e^{-L / l_{2}}\right)}{\bar{J}_{s, \mathrm{I}}+B_{2} l_{2}\left(1-e^{-L / l_{2}}\right)} .
$$

\subsection{Calculations}

Within the framework of density functional theory $[43,44]$, we solve the quantum mechanical scattering problem [120] for a general two terminal $\mathcal{L}|\mathcal{S}| \mathcal{R}$ configuration consisting of an $\mathrm{NM} \mid \mathrm{NM}^{\prime}$ scattering region $(\mathcal{S}$ ) embedded between ballistic left $(\mathcal{L})$ and right $(\mathcal{R})$ Au leads using a wave-function matching (WFM) method [47] implemented $[45,48]$ with a tight-binding (TB) muffin-tin orbital (MTO) basis in the atomic spheres approximation (ASA) [51, 74, 75] and generalized to include spin-orbit coupling and noncollinearity $[46,72]$ as well as temperature induced lattice and spin disorder [66,67]. The solution yields the scattering matrix $S$, from which we can directly calculate the conductance, as well as the full quantum mechanical wave function throughout the scattering region from which we can calculate position dependent charge and spin currents [32, 117]. Atomic sphere (AS) potentials for $\mathrm{Au}, \mathrm{Pt}$ and Pd are generated using the Stuttgart TB-LMTO code. Scattering calculations are carried out with an $s p d$ orbital basis and two center terms in the SOC Hamiltonian [46, 117] with tests carried out with three center terms. In all the calculations that follow, the transport direction is along $z$ and the atomic layers correspond to fcc [111] planes. 


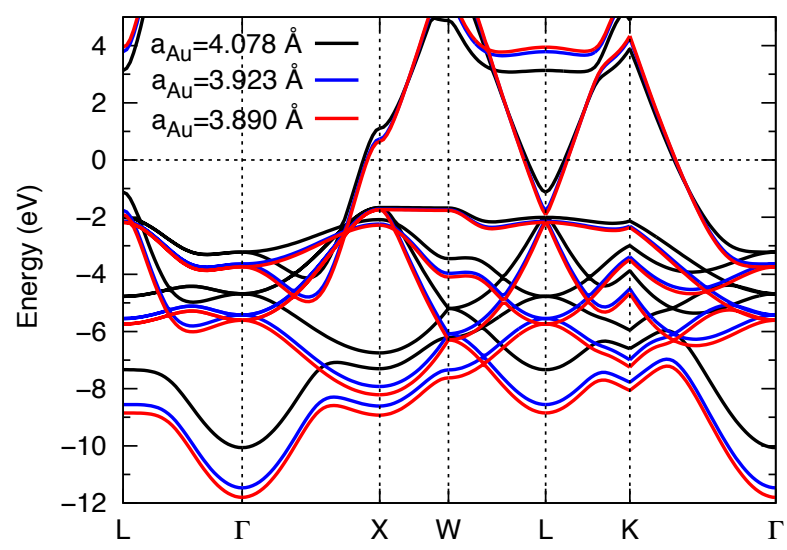

Figure 3.6: Band structure for uncompressed Au (black) and for Au compressed to have the lattice constant of Pt (blue) and Pd (red).

\section{Lattice mismatch: supercells}

The lattice constant of Au is $4.078 \AA$, that of Pt $3.923 \AA$ and of Pd $3.890 \AA$. To construct $\mathrm{Au} \mid \mathrm{Pt}$ and $\mathrm{Au} \mid \mathrm{Pd}$ bilayers, the unit cell areas of the two materials should be equal. This could be achieved by using lateral supercells $[30,32,46]$. Because of its simple nature, the electronic structure of Au does not change qualitatively close to the Fermi energy (shown in Fig. 3.6) when Au is compressed to make it match the lattice constants of Pd and Pt. Because it makes it much easier to study how modelling disorder in a lateral supercell depends on the supercell size, we instead adopt this simpler procedure. The effect of this approximation on the interface parameters is explicitly tested with lattice matched lateral supercells towards the end of this chapter in Table 3.1. In Sec. 2.3.1, we studied the dependence of the spin flip diffusion length and spin Hall angle in Pt on the lateral supercell size used to model thermal disorder. We concluded that a $7 \times 7$ supercell was sufficient to obtain adequately converged spin currents. For the bilayer calculations presented in this chapter, we have tested how spin currents passing through a Au|Pt interface depend on the supercell size and found that $7 \times 7$ is sufficient to describe interface parameters as well. Thus all the calculations presented in the Results section of this chapter are carried out with $7 \times 7$ supercells. The Brillouin zone (BZ) sampling used for this supercell is $32 \times 32 k$ points corresponding to an equivalent sampling of $224 \times 224 k$ points for a $1 \times 1$ unit cell.

\section{Thermal disorder}

A frozen thermal disorder scheme $[66,67]$ is used to model the $\mathrm{NM} \mid \mathrm{NM}^{\prime}$ bilayer systems at finite temperatures in the range $200-400 \mathrm{~K}$. Atoms are displaced from their equilibrium lattice positions following an uncorrelated Gaussian distribution 
that is characterized in terms of a root mean square displacement $\Delta$. For each material ( $\mathrm{NM}=\mathrm{Au}, \mathrm{Pd}, \mathrm{Pt})$ and temperature $T, \Delta_{\mathrm{NM}}(T)$ is chosen so as to reproduce the experimental resistivity at that temperature [73]. On constructing the $\mathrm{NM} \mid \mathrm{NM}^{\prime}$ bilayer, $\Delta_{\mathrm{NM}}(T)$ and $\Delta_{\mathrm{NM}^{\prime}}(T)$ are used for each material to generate multiple configurations with the required thermal disorder at temperature $T$. All the data presented result from averaging over 20 different configurations of disorder.

\subsection{Results}

As an illustration of the scheme that was described in the Methods section, we present results for the AulPt interface at $300 \mathrm{~K}$ : for $A R_{\mathrm{I}}$ in Sec. 3.4.1, for $\delta$ in Sec. 3.4.2, for the interface SHE in Sec. 3.4.3 and for $\Theta_{\mathrm{I}}$ from the ISHE in Sec. 3.4.4. The effect of three center terms on the extracted parameters is examined in Sec. 3.4.4. The $\mathrm{Au} \mid \mathrm{Pt}$ interface parameters are compared to those calculated for $\mathrm{Au} \mid \mathrm{Pd}$ at $300 \mathrm{~K}$ in Sec. 3.4.5. Finally, the dependence of $A R_{\mathrm{I}}, \delta$ and $\Theta_{\mathrm{I}}$ on temperature is presented in Sec. 3.4.6.

\subsubsection{Au|Pt: interface resistance}

The interface resistance $A R_{\mathrm{Au} \mid \mathrm{Pt}}$ is extracted in a two step procedure. We first calculate the total resistance for a symmetric Pt|Au|Pt trilayer embedded between ballistic Au leads for a variable length $L_{\mathrm{Pt}}$ of Pt and fixed length of $\mathrm{Au}, L_{\mathrm{Au}}=45 \mathrm{~nm}$. Both $L_{\mathrm{Pt}}$ and $L_{\mathrm{Au}}$ should be much longer than the respective mean free paths so that the total areal resistance for the scattering region can be expressed in terms of the series resistor model, sketched at the top of Fig. 3.7, as

$$
\begin{aligned}
A R\left(L_{\mathrm{Pt}}, L_{\mathrm{Au}}\right)= & \rho_{\mathrm{Pt}} L_{\mathrm{Pt}}+\rho_{\mathrm{Au}} L_{\mathrm{Au}}+2 A R_{\mathrm{Au} \mid \mathrm{Pt}} \\
& +2 A R_{\mathrm{Pt} \mid \mathrm{Au}(\mathrm{b})}+1 / G_{\mathrm{Sh}} .
\end{aligned}
$$

Here, $A R_{\mathrm{Au} \mid \mathrm{Pt}} \equiv A R_{\mathrm{I}}$ is the interface resistance we are interested in, $A R_{\mathrm{Pt} \mid \mathrm{Au}(\mathrm{b})}$ is the interface resistance between Pt and the ballistic Au lead, and $G_{\mathrm{Sh}}$ is the Sharvin conductance of the Au lead. In separate calculations for a variable thickness $L_{\mathrm{Pt}}$ of Pt embedded between Au leads, shown in Fig. 3.8, the total areal resistance

$$
A R\left(L_{\mathrm{Pt}}\right)=\rho_{\mathrm{Pt}} L_{\mathrm{Pt}}+\left\{2 A R_{\mathrm{Pt} \mid \mathrm{Au}(\mathrm{b})}+1 / G_{\mathrm{Sh}}\right\}
$$

is determined. Fitting $A R\left(L_{\mathrm{Pt}}\right)$ to (3.17) yields $\rho_{\mathrm{Pt}}$ as the slope and the underlined terms as the intercept. A similar calculation for diffusive Au yields $\rho_{\mathrm{Au}}$. We subtract the contributions $2 A R_{\mathrm{Pt} \mid \mathrm{Au}(\mathrm{b})}+1 / G_{\mathrm{Sh}}$ as well as $\rho_{\mathrm{Au}} L_{\mathrm{Au}}$ from $A R\left(L_{\mathrm{Pt}}, L_{\mathrm{Au}}\right)$ and plot the remainder, $\rho_{\mathrm{Pt}} L_{\mathrm{Pt}}+2 A R_{\mathrm{Au} \mid \mathrm{Pt}}$, in Fig. 3.7. Linear fitting yields the $L_{\mathrm{Pt}}=0$ intercept $A R_{\mathrm{Au} \mid \mathrm{Pt}}=0.54 \pm 0.03 \mathrm{f} \Omega \mathrm{m}^{2}$ at $300 \mathrm{~K}$. 


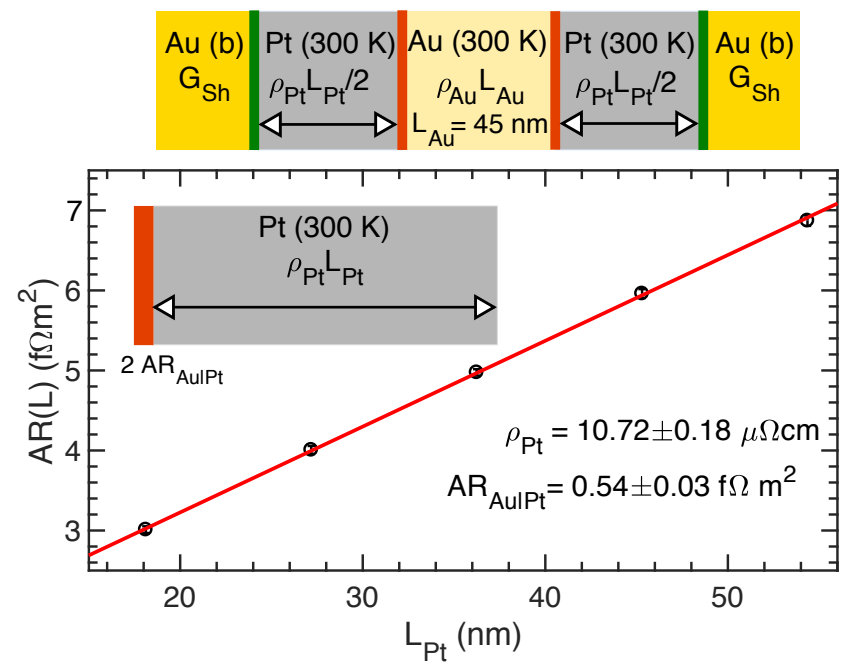

Figure 3.7: Total resistance of a diffusive $\mathrm{Pt}|\mathrm{Au}| \mathrm{Pt}$ trilayer, $\operatorname{Pt}\left(L_{\mathrm{Pt}} / 2\right)\left|\operatorname{Au}\left(L_{\mathrm{Au}}\right)\right| \operatorname{Pt}\left(L_{\mathrm{Pt}} / 2\right)$ sandwiched between ballistic $\mathrm{Au}$ leads as a function of the total Pt thickness $L_{\mathrm{Pt}}$ for a fixed Au thickness $L_{\mathrm{Au}}=45 \mathrm{~nm}$. To extract $A R_{\mathrm{Au} \mid \mathrm{Pt}}$, contributions from diffusive $\mathrm{Au}$ (pale yellow), $G_{\mathrm{Sh}}$ (yellow) and the interface resistance between ballistic $\mathrm{Au}$ and $\mathrm{Pt}$ (green) are calculated separately (see Fig. 3.8) and subtracted. $\rho_{\mathrm{Au}}$ is also determined separately. The intercept of $A R(L)$ at $L=0$ yields $A R_{\mathrm{Au|Pt}}$.

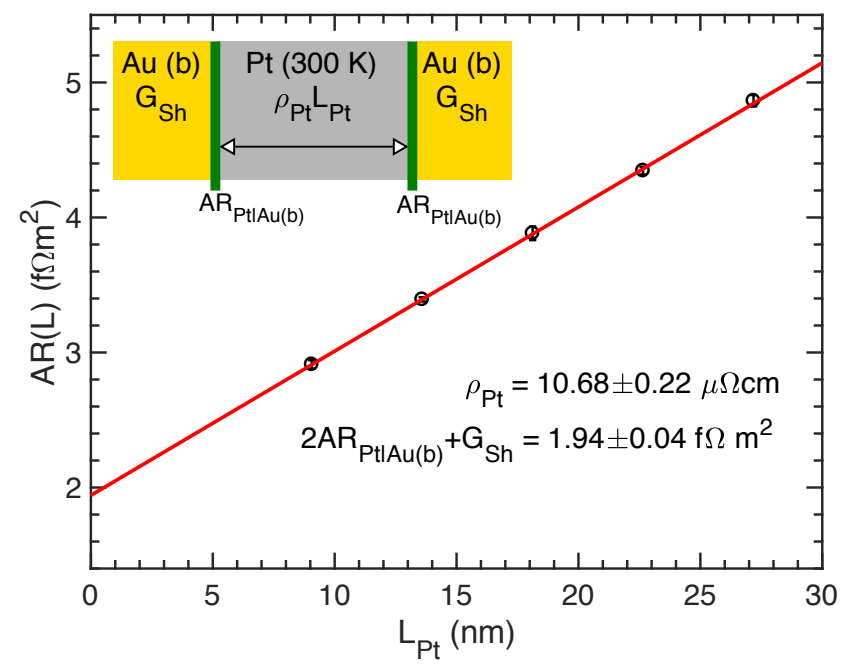

Figure 3.8: Total resistance for diffusive Pt sandwiched between ballistic(b) Au leads as a function of the Pt thickness $L_{\mathrm{Pt}}$. A linear fit AR(L) yields $\rho_{\mathrm{Pt}}$ as the slope; the intercept is a sum of interface and Sharvin contributions. 


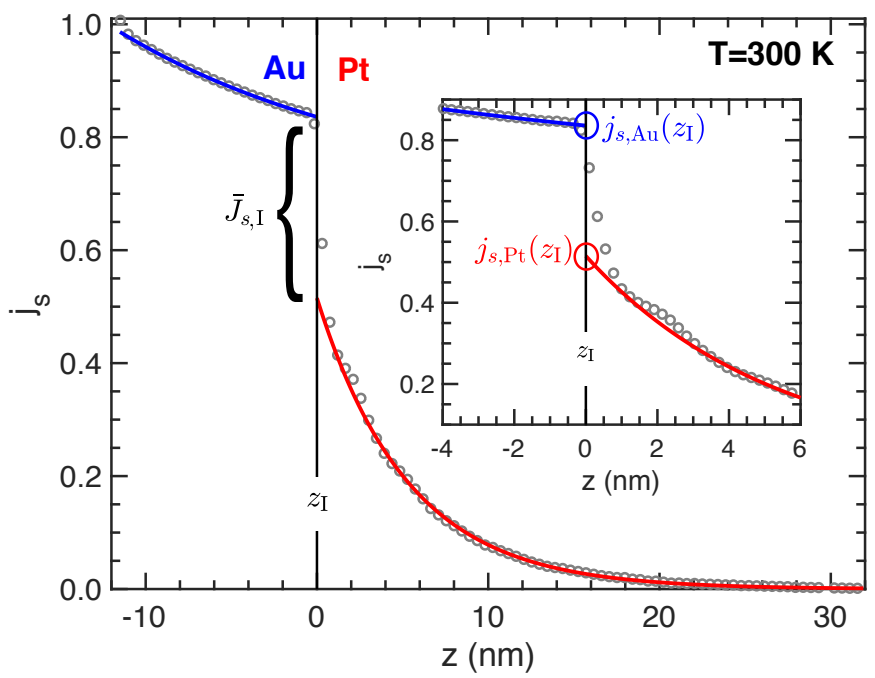

Figure 3.9: A fully polarized spin-current $j_{s}$ injected at $300 \mathrm{~K}$ from the left lead into a $\mathrm{Au}(10) \mid \operatorname{Pt}(30)$ bilayer sandwiched between Au leads decays exponentially in $\mathrm{Au}$ and in Pt. The numbers in brackets are lengths in $\mathrm{nm}$. The solid lines indicate fits for $j_{s}$ in individual layers using (3.6). The change in the spin current in the vicinity of the interface that is not "bulk-like" is denoted $\bar{J}_{s, \mathrm{I}}$. (Inset) Exploded view of the interface. $j_{s, \mathrm{Au}}\left(z_{\mathrm{I}}\right)$ and $j_{s, \mathrm{Pt}}\left(z_{\mathrm{I}}\right)$ indicate the values of the bulk spin currents extrapolated to $z_{\mathrm{I}}$ from the Au side and Pt side, respectively. $\delta$ is extracted using these values and (3.11).

\subsubsection{Au|Pt: $\delta$}

To calculate $\delta$ for a AulPt interface, we inject a fully spin-polarized current from a ballistic Au lead into a Au|Pt bilayer sandwiched between Au leads. The diffusive Au slab into which the spins are injected should be thick enough to avoid artifacts arising from ballistic transport but sufficiently thin so that a substantial spin current still enters Pt after spin flipping has occurred at the interface. We carried out tests with various lengths of $\mathrm{Au}$ (50, 100, 150 layers) keeping Pt fixed at 150 layers and found that the final results for $\delta$ were not affected by this choice. The results presented here are for 50 layers $(\sim 10 \mathrm{~nm})$ of Au and 150 layers $(\sim 30 \mathrm{~nm})$ of $\mathrm{Pt}$ that we denote $\mathrm{Au}(10) \mid \mathrm{Pt}(30)$. Both $\mathrm{Au}$ and $\mathrm{Pt}$ are modelled at $300 \mathrm{~K}$ using the rms displacements discussed in Sec. 3.3. The left lead is made to be "half-metallic" by lifting the bands of one spin channel above the Fermi energy, thus a fully polarized spin current flows into the bilayer. As shown in Fig. 3.9, the spin current decays rather slowly in Au reflecting the large value we found for $l_{\mathrm{Au}}$. At the AulPt interface, we see a sharp decrease in the spin-current as it enters Pt which is a clear indication of spin-memory loss. The spin current then decays exponentially towards zero in Pt. We fit $j_{s}(z)$ in Au and Pt using (3.3), giving values of the spin current close to 


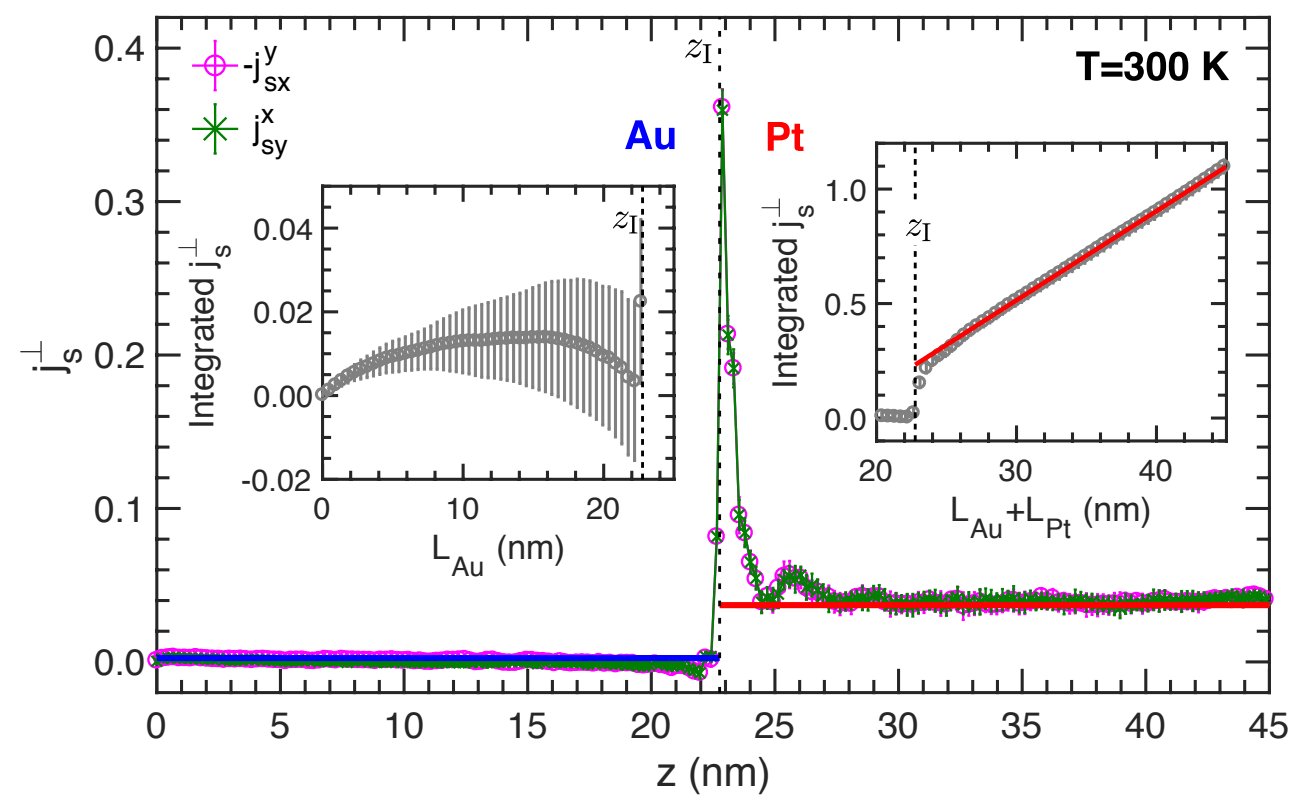

Figure 3.10: SHE in a $\mathrm{Au}(20) \mid \operatorname{Pt}(20)$ bilayer at $300 \mathrm{~K}$ embedded between $\mathrm{Au}$ and Pt leads on the left and right, respectively. Transverse spin currents $-j_{s x}^{y}$ and $j_{s y}^{x}$ are shown as pink circles and green crosses. The blue and red horizontal lines show the values of the bulk spin Hall angles of $\mathrm{Au}, \Theta_{\mathrm{Au}}=0.0025$ and of Pt, $\Theta_{\mathrm{Pt}}=0.037 \pm 0.001$ calculated separately for bulk diffusive Au and Pt. (Left inset) Integrated transverse spin current in Au. (Right inset) Integrated transverse spin current in Pt. The solid red line indicates the fit obtained from the integrated spin current in Pt. The intercept at $z_{\mathrm{I}}$ shows the contribution from the interface.

the interface less weight, and extrapolate the fitted curves to the interface at $z_{\mathrm{I}}=0$.

We rewrite (3.11) for the AulPt interface as

$$
\frac{j_{s, \mathrm{Au}}\left(z_{\mathrm{I}}\right)}{j_{s, \mathrm{Pt}}\left(z_{\mathrm{I}}\right)}=\cosh \delta+\delta \sinh \delta \frac{\rho_{\mathrm{Pt}} l_{\mathrm{Pt}}}{A R_{\mathrm{I}}}
$$

In Sec. 2.3.1, we extracted a value of $l_{\mathrm{Pt}}=5.25 \pm 0.05 \mathrm{~nm}$ at $\mathrm{T}=300 \mathrm{~K}$. As shown in Fig. 3.8, $\rho_{\mathrm{Pt}}=10.7 \pm 0.2 \mu \Omega \mathrm{cm}$. The only unknown in (3.18) is $\delta$. On substituting all the other parameters in (3.18), we extract $\delta$ using a numerical root finder. For this $\mathrm{Au} \mid \mathrm{Pt}$ interface at $300 \mathrm{~K}$, we find $\delta=0.62 \pm 0.03$. The error bar is evaluated by taking into account the spread of all the input parameters as described by their respective error bars. 


\subsubsection{Au|Pt: interface SHE}

In Fig. 3.4, we discussed the qualitative behaviour of the spin Hall effect in the bulk of two nonmagnetic materials and how it abruptly changes at an interface. We now pass an unpolarized charge current through a $300 \mathrm{~K}$ diffusive $\mathrm{Au}(20) \mid \operatorname{Pt}(20)$ bilayer sandwiched between a ballistic Au lead on the left and Pt lead on the right in order to minimize interface contributions from the leads. Transverse spin currents $j_{s y}^{x}$ and $j_{s x}^{y}$ generated by the SHE in the two materials and at their interface are shown as pink circles and green crosses in Fig. 3.10. The horizontal blue and red lines show the values of the bulk spin Hall angles of $\mathrm{Au}, \Theta_{\mathrm{Au}}=0.0025$ and of Pt, $\Theta_{\mathrm{Pt}}=0.037 \pm 0.001$ determined separately. At short distances from the interface, spin currents are seen to obey their bulk behaviour in both materials. In a region of $\sim 5 \mathrm{~nm}$ about the interface at $z_{\mathrm{I}}$, we see clear deviations from bulk behaviour in both Au and Pt that culminate in a large interface spin Hall contribution. To quantitatively describe this sharp peak, we integrate the transverse spin currents in the bilayer starting from the interface with the left Au lead at $z=0$ up to the $\mathrm{Au} \mid \mathrm{Pt}$ interface at $z=z_{\mathrm{I}}=L_{\mathrm{Au}}$ (left-hand inset) and onward to the interface with the right Pt lead at $z=L_{\mathrm{Au}}+L_{\mathrm{Pt}}$ (right-hand inset).

The integral $\int_{0}^{L_{\mathrm{Au}}} d z\left(j_{s y}^{x}-j_{s x}^{y}\right)$ plotted in the left-hand inset of Fig. 3.10 shows the integrated spin current increasing from zero up to a certain value of $L_{\mathrm{Au}}$ before decreasing again to essentially zero close to the interface at $z_{\mathrm{I}}$. If we add the total integrated contribution from $\mathrm{Au}$ and continue to integrate through Pt, the result is $\int_{0}^{L_{\mathrm{Au}}} d z\left(j_{s y}^{x}-j_{s x}^{y}\right)+\int_{z_{\mathrm{I}}}^{L_{\mathrm{Au}}+L_{\mathrm{Pt}}} d z\left(j_{s y}^{x}-j_{s x}^{y}\right)$ and it is shown in the right-hand inset. It can be fitted with a straight line whose slope is just the value we calculate independently for bulk Pt, $\Theta_{\mathrm{Pt}}=0.0371 \pm 0.001$. The finite intercept 0.22 yields the contribution from the interface spin Hall effect in units of nm. To extract a dimensionless interface SHA $\Theta_{\mathrm{I}}$, we make use of the charge currents generated by the inverse spin Hall effect in Au|Pt in the next section.

\subsubsection{Au|Pt: interface ISHE - $\Theta_{\mathrm{I}}$}

The spin current $j_{s}(z)$ shown in Fig. 3.9 is polarized in the $-x$ direction ${ }^{*}$. A consequence of the (inverse) spin Hall effect is that such a transversely polarized spin current induces a charge current $j_{c}^{\perp}(z)$ which is given by the vector product of the current and polarization directions. Its magnitude is shown in Fig. 3.11. Far from the interface, this charge current should simply depend on the material specific SHA $\Theta_{i}$ as $\Theta_{i} j_{s i}(z)$. By comparing this product with the explicitly calculated $j_{c}^{\perp}(z)$, we can identify departures from the expected bulk behavior and attribute them to the interface. We already fitted $j_{s i}(z)$ in Fig. 3.9 to (3.3) resulting in the blue and red solid lines. In Sec. 2.3.4, we calculated $\Theta_{\mathrm{Pt}}=0.037 \pm 0.001$ (using 2-center

\footnotetext{
${ }^{*}$ In principle, $\delta$ could depend on this polarization direction; in practice we find this dependence to fall within the $5 \%$ accuracy with which $\delta$ was determined.
} 


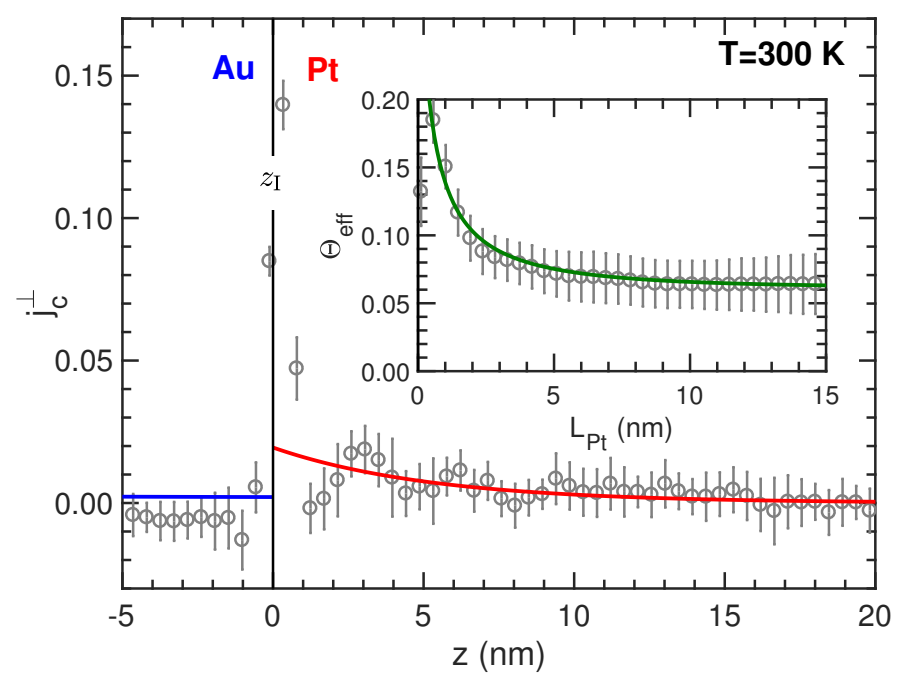

Figure 3.11: $z$ dependence of the ISHE charge current in the $y$ direction (open circles) induced by the spin current polarized in the $-x$ direction shown in Fig. 3.9. The solid blue and red lines indicate the fitted $j_{s, \mathrm{Au}}$ and $j_{s, \mathrm{Pt}}$ from Fig. 3.9 multiplied by the bulk spin Hall angles: $\Theta_{\mathrm{Au}}=0.0025$ and $\Theta_{\mathrm{Pt}}=0.037$ respectively. (Inset) Effective SHA $\Theta_{\text {eff }}$ calculated from the spin and charge currents integrated from $z_{\mathrm{I}}=0$ to $z=L_{\mathrm{Pt}}$. The solid green line is the fit obtained using (3.15). 
terms and spd orbitals) at $300 \mathrm{~K}$. Using the same procedure, we find the spin Hall angle for $\mathrm{Au}$ to be $\Theta_{\mathrm{Au}}=0.0025$ at $300 \mathrm{~K}$. The solid blue and red lines in Fig. 3.11 represent $\Theta_{\mathrm{Au}} j_{s, \mathrm{Au}}(z)$ and $\Theta_{\mathrm{Pt}} j_{s, \mathrm{Pt}}(z)$. On the Pt side of the interface, the calculated $j_{c}^{\perp}(z)$ approaches the expected bulk value $\Theta_{\mathrm{Pt}} j_{s, \mathrm{Pt}}(z)$ as we move away from the interface. Right at the interface, a high and narrow $j_{c}^{\perp}(z)$ signals an interface SHA much larger than the Pt bulk SHA. In Au it seems that the transverse charge current $j_{c}^{\perp}(z)$ has not yet reached its asymptotic bulk value. Although the spin current injected from the lead is still almost fully polarized at the $\mathrm{Au|Pt}$ interface because of the weak spin-flipping in Au, a very small ISHE bulk charge current, shown by the blue line in Fig. 3.11, is expected because of the very small value of $\Theta_{\mathrm{Au}}=0.0025$. However, the actual $j_{c}^{\perp}(z)$ is seen to be negative indicating that the interface has an influence extending further into Au. More work is required to establish how the interface influences the SHE and ISHE in Au.

To extract $\Theta_{\mathrm{I}}$, we integrate the calculated spin current and the corresponding ISHE-induced charge current from $z_{\mathrm{I}}=0$ up to $L_{\mathrm{Pt}}$ in Pt. The resulting effective SHA, $\Theta_{\text {eff }}$, is plotted in the inset to Fig 3.11 as a function of $L_{\mathrm{Pt}}$. Using (3.15), we fit $\Theta_{\text {eff }}$ to obtain $\Theta_{\mathrm{I}}=0.35 \pm 0.10$, almost 10 times larger than the bulk Pt SHA.

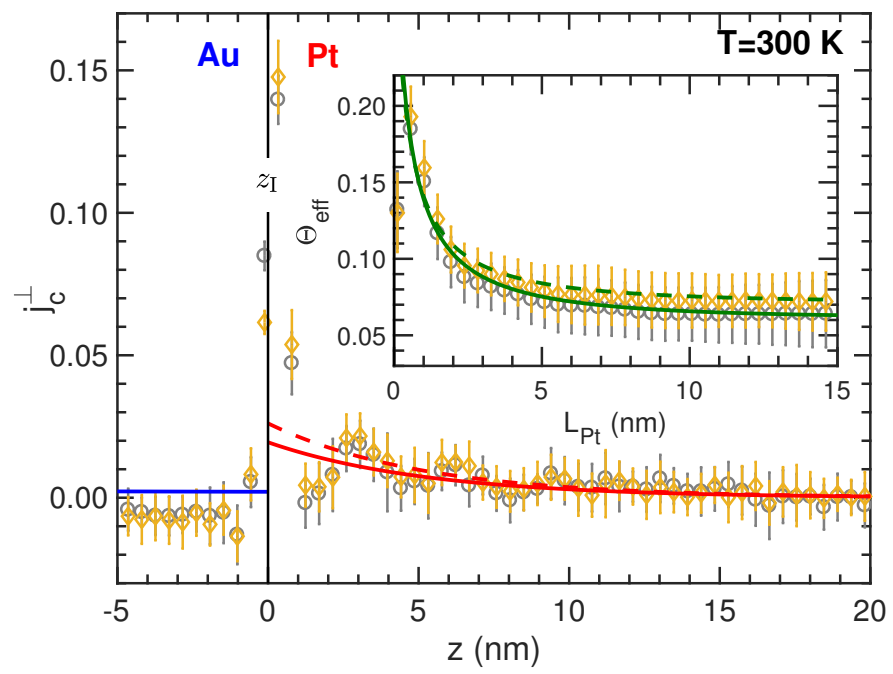

Figure 3.12: Comparison of the ISHE induced transverse charge current $j_{c}^{\perp}$ in the $y$ direction calculated with two center terms (grey circles) and three center terms (yellow diamonds) for a $\mathrm{Au}(10) \mid \mathrm{Pt}(30)$ bilayer embedded between ballistic $\mathrm{Au}$ leads at $300 \mathrm{~K}$. The solid and dashed red lines indicate the fitted $j_{s, \mathrm{Pt}}(z)$ multiplied by the bulk spin Hall angles $\Theta_{\mathrm{Pt}}=0.037$ and $\Theta_{\mathrm{Pt}}=0.050$ obtained using two and and three center terms, respectively. (Inset) Effective SHA $\Theta_{\text {eff }}$ calculated using two and three center terms, in grey circles and yellow diamonds, respectively. The solid and dashed green lines indicate the corresponding fits obtained using (3.15). 


\section{Three center terms}

In Sec. 2.3.4, we compared $l_{\mathrm{Pt}}$ and $\Theta_{\mathrm{Pt}}$ obtained using two and three center terms in the SOC part of the Hamiltonian. On including three center terms, $l_{\mathrm{Pt}}$ decreased by $5 \%$ from 5.25 to $4.96 \mathrm{~nm}$ while $\Theta_{\mathrm{Pt}}$ increased by $35 \%$, from 0.037 to 0.050 . For the present $\mathrm{Au} \mid \mathrm{Pt}$ bilayer, we find that the spin current and spin-memory loss do not depend on the three center terms. In Fig. 3.12, we compare the ISHE induced charge current for the two cases. A slight difference in $j_{c}^{\perp}$ in the vicinity of the interface is visible. By plotting the effective SHA in the inset to Fig. 3.12, we observe that $\Theta_{\text {eff }}$ calculated with three center terms (yellow diamonds) appears simply shifted compared to the original data (grey circles) by virtue of the higher value of $\Theta_{\mathrm{Pt}}$. Indeed by fitting to (3.15), $\Theta_{\mathrm{I}}=0.33 \pm 0.11$ compared to $\Theta_{\mathrm{I}}=0.35 \pm 0.10$ obtained using two-center terms. Thus $\Theta_{\mathrm{I}}$ is not affected by the choice of two or three center terms within the accuracy of the calculations.

\subsubsection{Au|Pt vs Au|Pd}

Now that we have described how $A R_{\mathrm{I}}, \delta$ and $\Theta_{\mathrm{I}}$ are determined for $\mathrm{Au} \mid \mathrm{Pt}$, we repeat the procedure for the Au|Pd interface and compare the results for the two interfaces. First, we extract the bulk parameters for Pd that are needed for the fits that will result in the interface parameters. We find $l_{\mathrm{Pd}}=7.06 \pm 0.02 \mathrm{~nm}$ and $\Theta_{\mathrm{Pd}}=0.035 \pm$ 0.001 corresponding to the experimental resistivity of $\mathrm{Pd}, \rho_{\mathrm{Pd}}=10.8 \mu \Omega \mathrm{cm}$ at 300 $\mathrm{K}$ [73].

In Fig. 3.13, we compare the effect of spin-flip diffusion in AulPt (grey circles) and $\mathrm{Au} \mid \mathrm{Pd}$ (black diamonds) bilayers at room temperature. Small differences are visible in the decrease of $j_{s}(z)$ in Au for the two systems. One reason is that the interface reflectivity determining the coefficient $A_{i}$ of the increasing exponential term in (3.6) is different, as we will see below. In addition, the lattice constant of $\mathrm{Au}$ is matched to that of Pt or Pd so is not the same for the two bilayers. In the vicinity of the interface, the rapid decay of $j_{s}(z)$ is more prominent and much sharper in Pt than in Pd, as shown in the inset to Fig. 3.13. By fitting $j_{s}(z)$ to (3.6), we obtain $j_{s, \mathrm{Au}}\left(z_{\mathrm{I}}\right)$ and $j_{s, \mathrm{Pd}}\left(z_{\mathrm{I}}\right)$. A value of $A R_{\mathrm{Au} \mid \mathrm{Pd}}=0.81 \pm 0.05 \mathrm{f} \Omega \mathrm{m}^{2}$ is obtained using the procedure described in Sec. 3.4.1; this is much larger than the value $0.54 \pm 0.03 \mathrm{f} \Omega \mathrm{m}^{2}$ we found for Au|Pt. By substituting all the input parameters and their uncertainities into (3.11), a value of $\delta=0.43 \pm 0.02$ is extracted numerically.

In Fig. 3.14, we compare the ISHE induced charge current $j_{c}^{\perp}$ for Au|Pt (grey circles) and $\mathrm{Au} \mid \mathrm{Pd}$ (black diamonds) at $\mathrm{T}=300 \mathrm{~K}$. The peak around $z_{\mathrm{I}}$ coming from the interface ISHE described by $\Theta_{\mathrm{I}}$ is significantly lower for Au|Pd than for Au|Pt. By fitting $\Theta_{\text {eff }}$, we find $\Theta_{\mathrm{I}}=0.21 \pm 0.03$ for $\mathrm{Au} \mid \mathrm{Pd}$. There are a few other interesting features. Beyond $3 \mathrm{~nm}$ from the interface, $j_{c}^{\perp}(z)$ in Pd and Pt appear almost identical. Given that we find $\Theta_{\mathrm{Pd}}=0.035$ is only $5 \%$ smaller than $\Theta_{\mathrm{Pt}}=0.037$, this is not surprising. On the other hand, $j_{c}^{\perp}$ in Au gradually decreases towards a 


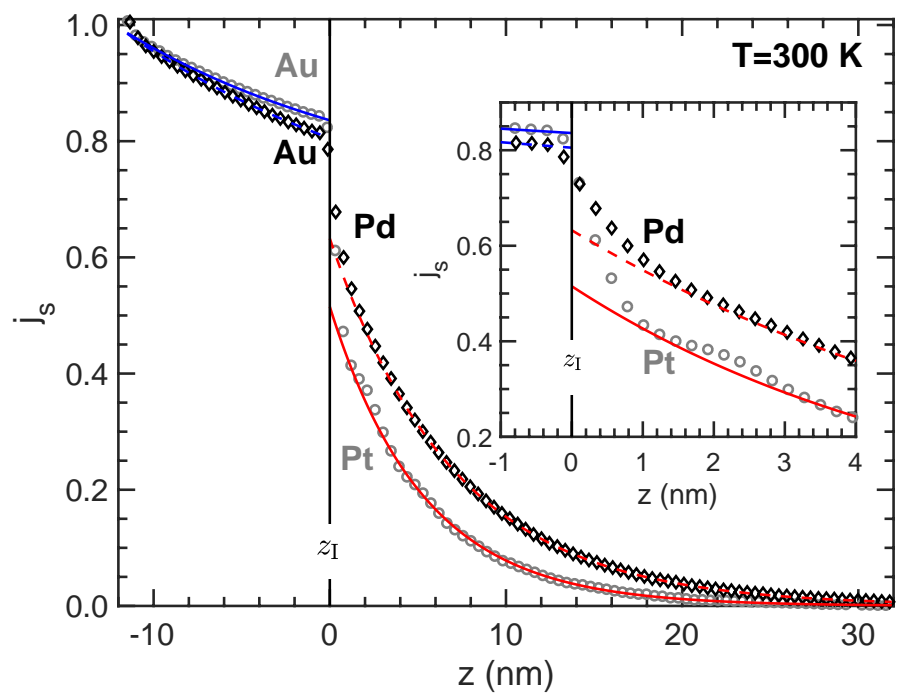

Figure 3.13: Calculated spin-current density for a $\mathrm{Au}(10) \mid \mathrm{Pt}(30)$ bilayer (grey circles) vs $\mathrm{Au}(10) \mid \operatorname{Pd}(30)$ (black diamonds) embedded between ballistic Au leads at $300 \mathrm{~K}$. The solid and dashed lines indicate the fits for $j_{s}$ in individual layers fitted by using (3.6) for $\mathrm{Au|Pt}$ and $\mathrm{Au} \mid \mathrm{Pd}$ bilayers respectively. Inset: a closer look at the rapid decay of the spin current in the vicinity of the $\mathrm{Au} \mid \mathrm{Pt}$ and $\mathrm{Au} \mid \mathrm{Pd}$ interfaces.

small positive value away from the AulPd interface. This is in contrast to the small negative value we see for Au in the Au|Pt bilayer. In neither case has $j_{c}^{\perp}$ reached its asymptotic value in Au, consistent with the assumption that the SHE and ISHE in $\mathrm{Au}$ are strongly influenced by the interface SHE. Determining the lengthscale over which this takes place requires additional study.

\subsubsection{Temperature dependence of the interface parameters}

While the bulk parameters $\rho_{\mathrm{Pt}}, 1 / l_{\mathrm{Pt}}$ and $\Theta_{\mathrm{Pt}}$ increase linearly with temperature $[32,59,67,73]$, nothing is known about how interface parameters behave as a function of temperature. We determined the Au|Pt interface parameters at 200 and $400 \mathrm{~K}$ in addition to the results demonstrated for $300 \mathrm{~K}$ and plot the resulting temperature dependence of $R_{\mathrm{I}}, \delta$ and $\Theta_{\mathrm{I}}$ in Fig. 3.15. Within the error bars of the calculations, all three parameters remain constant between 200 and $400 \mathrm{~K}$.

\subsection{Summary and Conclusions}

In this Chapter, we have described a scheme to extract $R_{\mathrm{I}}$ and $\delta$ for nonmagnetic $\mathrm{Au} \mid \mathrm{Pt}$ and $\mathrm{Au} \mid \mathrm{Pd}$ interfaces at finite temperatures from local spin and charge currents calculated from the results of first principles fully relativistic scattering cal- 


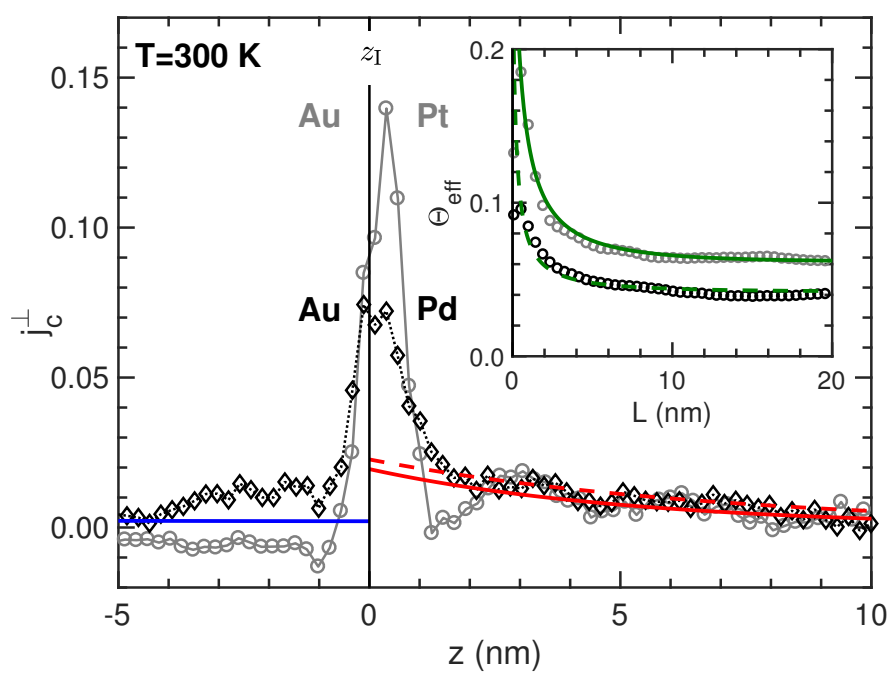

Figure 3.14: Calculated transverse charge current along y direction due to ISHE for a $\mathrm{Au}(10) \mid \mathrm{Pt}(30)$ (grey circles) vs $\mathrm{Au}(10) \mid \mathrm{Pd}(30)$ (black diamonds) bilayer embedded between ballistic Au leads at $300 \mathrm{~K}$. (Inset) Effective SHA $\Theta_{\text {eff }}$ calculated for $\mathrm{Pt}$ and Pd interfaced with $\mathrm{Au}$, in grey circles and black diamonds respectively. The solid and dashed green lines indicate the fits obtained for Pt and Pd respectively using Eq. 3.15. 

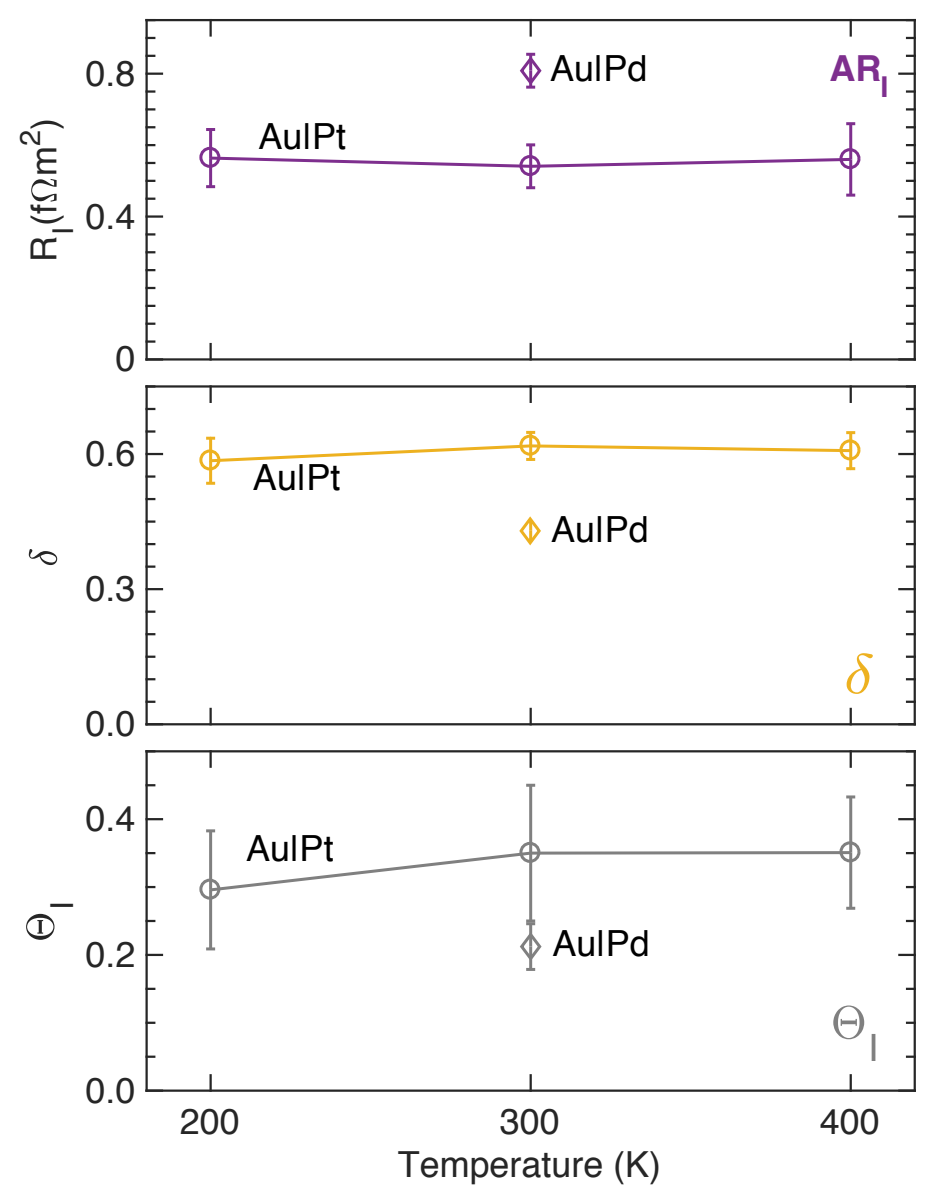

Figure 3.15: Temperature dependence of the interface parameters $R_{\mathrm{I}}$ (purple), $\delta$ (yellow) and $\Theta_{\mathrm{I}}$ (grey) for a Au|Pt interface (circles). The same set of parameters for $\mathrm{Au} \mid \mathrm{Pd}$ at $300 \mathrm{~K}$ are included (diamonds). 
Table 3.1: (Upper) Bulk transport parameters: resistivity $\rho$, spin-flip diffusion length $l_{\mathrm{sf}}$ and spin-Hall angle $\Theta_{\mathrm{sH}}$ for Pt and Pd. (Lower) Interface transport parameters: interface resistance $A R_{\mathrm{Au} \mid \mathrm{NM}}$, spin-memory loss parameter $\delta$ and interface spin-Hall angle $\Theta_{\mathrm{Au} \mid \mathrm{NM}}$ for $\mathrm{Au} \mid \mathrm{Pd}$ and $\mathrm{Au} \mid \mathrm{Pt}$ interfaces at $300 \mathrm{~K}$. For the $\mathrm{Au} \mid \mathrm{Pt}$ case, two interfaces are considered: a pseudomorphic interface for which $a_{\mathrm{Au}}$ is chosen to be equal to $a_{\mathrm{Pt}}$ and a fully relaxed interface between equilibrium Au and Pt.

\begin{tabular}{l|c|cc}
\hline \hline & Pd & \multicolumn{2}{|c}{ Pt } \\
\hline$\rho(\mu \Omega \mathrm{cm})$ & $10.8 \pm 0.1$ & \multicolumn{2}{|c}{$10.7 \pm 0.2$} \\
$l_{\mathrm{sf}}(\mathrm{nm})$ & $7.06 \pm 0.02$ & \multicolumn{2}{|c}{$5.25 \pm 0.05$} \\
$\Theta_{\mathrm{sH}}$ & $0.035 \pm 0.001$ & $0.037 \pm 0.001$ \\
\hline & $a_{\mathrm{Au}}=a_{\mathrm{Pd}}$ & $a_{\mathrm{Au}}=a_{\mathrm{Pt}}$ & $a_{\mathrm{Au}}=a_{\mathrm{Au}}$ \\
\hline$A R_{\mathrm{Au} \mid \mathrm{NM}}\left(f \Omega \mathrm{m}^{2}\right)$ & $0.81 \pm 0.05$ & $0.54 \pm 0.03$ & $0.81 \pm 0.04$ \\
$\delta$ & $0.43 \pm 0.02$ & $0.62 \pm 0.03$ & $0.81 \pm 0.05$ \\
$\Theta_{\mathrm{Au} \mid \mathrm{NM}}$ & $0.21 \pm 0.03$ & $0.35 \pm 0.10$ & $0.46 \pm 0.18$ \\
\hline \hline
\end{tabular}

culations. We also extracted $\Theta_{\mathrm{I}}$ using a scheme proposed by Wang and coworkers [32]. Table 3.1 summarizes the results for the Pd and Pt bulk parameters as well as the interface parameters for the corresponding interfaces with $\mathrm{Au}$ at $\mathrm{T}=300 \mathrm{~K}$. We found a substantial spin-memory loss and an interface SHA that is almost an order of magnitude larger than the bulk SHA for both interfaces even though we neglected the lattice mismatch.

To study the effect lattice mismatch has on the interface parameters, we calculate them for a (111) $\mathrm{Au} \mid \mathrm{Pt}$ interface where both $\mathrm{Au}$ and $\mathrm{Pt}$ have their equilibrium bulk volumes given by $a_{\mathrm{Au}}=4.078 \AA$ and $a_{\mathrm{Pt}}=3.923 \AA$. A (111) oriented $5 \times 5$ unit cell of Au matches with a similarly oriented $3 \sqrt{3} \times 3 \sqrt{3}$ unit cell of Pt to better than $0.02 \%$. The unit cells need to be rotated with respect to each other to make them coincide. For this fully relaxed Au|Pt geometry, we repeat our calculations at $300 \mathrm{~K}$ for $R_{\mathrm{I}}, \delta$ and $\Theta_{\mathrm{I}}$ and include the results in Table 3.1. All three interface parameters obtained with the Au lattice in equilibrium are larger than those obtained with compressed $\mathrm{Au}$; the interface is more pronounced. We attribute this to the lack of conservation of transverse momentum and greater $\mathbf{k} \rightarrow \mathbf{k}^{\prime}$ scattering in the absence of commensurability.

Because of their relatively weak effective SOC, the free electron like metals $\mathrm{Cu}$, $\mathrm{Ag}$ and $\mathrm{Au}$ are often used as spacer layers in spin-pumping and related experiments to suppress the magnetization induced in Pd and Pt by proximity to a magnetic layer, the so-called "magnetization proximity effect" (MPE); this is considered to have a significant influence on spin transport through interfaces [108, 121]. While the role of the MPE and whether a spacer layer modifies the interface effects are still being debated [29, 35, 122-124], our findings show that when nonmagnetic 
spacer layers are introduced, additional interface parameters must also be introduced to describe the additional effects such as spin memory loss and interface SHE at the new interfaces, even when these are between nonmagnetic materials. Many experiments use materials like Ta as capping or seeding layers adjacent to Pt $[36,38]$. For small thicknesses of Pt, an interface with Ta may also lead to an enhanced interface SHE and spin memory loss. Not taking this into consideration will most likely influence the values of "bulk" parameters extracted for Pt.

Experimental [112] and theoretical [113] studies have shown that nonmagnetic interfaces can generate spin currents and exert torques on neighbouring magnetic layers. This again points towards the importance of interface spin-orbit splitting for nonmagnetic interfaces and the large values of the interface SHA we observe support these studies. The interface SHA could be employed as a parameter that determines the efficiency of these spin currents.

Larger SOC in Pt compared to Pd leads to a relatively higher $\delta$ and $\Theta_{\mathrm{I}}$ for Au|Pt compared to $\mathrm{Au} \mid \mathrm{Pd}$. Here, it is important to note that the bulk spin Hall angles that we find for the two materials differ by only $5 \%$, but numerous studies suggest a larger SHA for Pt compared to Pd [12]. Along with the wide spread in values quoted for the bulk SHA, we suggest that identifying the role of the SML and the interface SHA is essential to reliably and reproducibly extract values of the SHA characteristic of bulk Pd and Pt.

For the $\mathrm{Au} \mid \mathrm{Pt}$ interface, we have seen that varying the temperature does not change the interface parameters. Schep et al. developed a model for the interface resistance in terms of a "ballistic" interface embedded in a diffusive scattering medium $[69,125]$. The temperature-independent behaviour we find for $R_{\mathrm{Au} \mid \mathrm{Pt}}$ requires scattering through the interface to remain specular for any given bilayer temperature. To explicitly prove this, we calculate $R_{\mathrm{Au|Pt}}$ in the absence of any thermal disorder, with and without SOC for the lattice matched (compressed Au) case. Compared to the room temperature value of $0.54 \pm 0.03 \mathrm{f} \Omega \mathrm{m}^{2}$ calculated using the procedure described in 3.4.1, we find $R_{\mathrm{Au} \mid \mathrm{Pt}}=0.63 \mathrm{f} \Omega \mathrm{m}^{2}$ with SOC and $0.56 \mathrm{f} \Omega \mathrm{m}^{2}$ without SOC using Schep's procedure.

Our results for $R_{\mathrm{I}}$ and $\delta$ for Au|Pd interfaces can be compared to those for ballistic $\mathrm{Cu} \mid \mathrm{Pd}$ ( $\mathrm{Cu}$ expanded to match the Pd lattice) interfaces estimated theoretically by Belashchenko et al [115]. By combining Schep et al.'s approach with circuit theory, they obtained $R_{\mathrm{I}}$ in the range of 0.70 to $0.85 \mathrm{f} \Omega \mathrm{m}^{2}$ and $\delta$ in the range 0.38 to 0.54 for ideal (001) oriented interfaces as well as for various rough (111) oriented interfaces obtained by intermixing. These results for a ballistic $\mathrm{Cu} \mid \mathrm{Pd}$ bilayer match very well with our results for diffusive $\mathrm{Au} \mid \mathrm{Pd}$ bilayer. Since $\mathrm{Au}$ and $\mathrm{Cu}$ have similar electronic structures, the temperature independence can indeed be traced back to ballistic case for $R_{\mathrm{I}}$ as well as $\delta$. More work is required to establish if such temperature independence is exhibited by other nonmagnetic interfaces. 


\section{4}

\section{Calculating interface transport parameters at finite temperatures: Ferromagnetic | Nonmagnetic interfaces*}

Spin-memory loss (SML) parameter $\delta$ is believed to be significant in experiments involving interfaces between ferromagnetic and nonmagnetic metals, especially heavy metals like Pt, it is more often than not neglected to avoid introducing too many unknown interface parameters in addition to often poorly known bulk parameters like the spin-flip diffusion length $l_{\mathrm{sf}}$. $\delta$ for these interfaces has only been determined directly at low temperatures. In this chapter, we calculate $\delta$ along with the interface resistance $R_{I}$ and the spin-asymmetry parameter $\gamma$ as a function of temperature for $\mathrm{Co} \mid \mathrm{Pt}$ and Py|Pt interfaces where Py is the ferromagnetic $\mathrm{Ni}_{80} \mathrm{Fe}_{20}$ alloy, permalloy. We use first-principles scattering theory to calculate the conductance as well as local charge and spin currents, modeling temperature-induced disorder with frozen thermal lattice and, for ferromagnetic materials, spin disorder within the adiabatic approximation. The bulk and interface parameters are extracted from the spin currents using the Valet-Fert model generalized to include SML.

"To be submitted as: K. Gupta, R.J.H. Wesselink, Z. Yuan, P.J. Kelly, Spin transport at finite temperatures: A first-principles study for Ferromagnetic|Nonmagnetic interfaces 


\subsection{Introduction}

A NM|FM interface between nonmagnetic (NM) and ferromagnetic (FM) metals is characterized in terms of an interface resistance $R_{I}$, a polarization $\gamma$ and a spin memory loss (SML) parameter $\delta$. These parameters are analogous to their bulk counterparts: resistivity $\rho$, a polarization (or spin-asymmetry) parameter $\beta$ that vanishes for nonmagnetic materials, and a spin-flip diffusion length (SDL) $l_{\mathrm{sf}}$. To describe the transport in noncollinearly aligned $\mathrm{FM}|\mathrm{NM}| \mathrm{FM}^{\prime}$ spin valves it is necessary to introduce an additional interface parameter, a complex, so-called spinmixing conductance $G_{\uparrow \downarrow}[55,122,126]$. In ferromagnetic materials the spin-orbit coupling (SOC) and conductivity polarization lead to a Hall effect in the absence of an external magnetic field that is characterized by the "anomalous Hall angle" $\Theta_{\mathrm{aH}}$. In heavy nonmagnetic elements the SOC gives rise to the spin Hall effect (SHE) $[11,12,19,20,119]$ whereby an electric current leads to the generation of a transverse spin current. The SHE is characterized in terms of the spin Hall angle (SHA) $\Theta_{\mathrm{sH}}$ that is the ratio of the spin current (measured in units of $\hbar / 2$ ) to the charge current (measured in units of the electron charge $-|e|$ ). For interfaces an interface SHA $\Theta_{\mathrm{sH}}^{I}$ can be defined by analogy [32]. Phenomenological theories ultimately aim to relate currents of charge $\mathbf{j}_{c}$ and spin $\mathbf{j}_{s \alpha}$ to gradients of the chemical potential $\mu_{c}$ and spin accumulation $\mu_{s \alpha}$ in terms of the above parameters but tell us nothing about the values of the parameters for particular materials or combinations of materials [17]. Here $\alpha$ labels the spin component.

It has turned out to be remarkably difficult to measure many of the parameters described above quantitatively $[10,17]$, especially at other than low temperatures. In particular, virtually nothing is known about the interface parameters $R_{I}, \gamma$ and $\delta$ at room temperature because, unless the sample cross sections are reduced by structuring, [7] the interface resistance is swamped by other resistances. The use of superconducting leads restricts studies to the low superconducting critical temperatures of commonly used metals like $\mathrm{Al}$ or $\mathrm{Nb}$ [6]. At these low temperatures transport parameters are strongly extrinsic but little is known about the nature of the bulk disorder that gives rise to diffuse transport. The situation with respect to interface disorder is even worse because so little is known about it on an atomic level.

Ten years ago only a handful of measurements had been made of the SDL [10] or of the SHA [11, 12]. The advent of nonlocal spin injection [21, 87, 89] and spinpumping (SP) $[22,23,28,90]$ techniques has allowed the SHA to be determined by means of the inverse SHE (ISHE). Alternatively, spin currents generated by the SHE can be used to drive the precession of a magnetization by the spin-transfer torque (STT) that is monitored using ferromagnetic resonance (FMR) [24, 88]. These innovations have changed the situation radically over the past ten years yielding a host of very disparate room temperature results for $l_{\mathrm{sf}}$ and $\Theta_{\mathrm{sH}}[11,12]$. The new measurement techniques work by introducing an interface through which spin 
must flow in order to be detected. Though attempts have been made to take the interface effects described above into account [29,35-38], this has yet to be done systematically by determining all parameters for consistent sets of samples and, if anything, has led to an increase in the spread of values reported for the key parameters for bulk Pt, $l_{\mathrm{sf}}^{\mathrm{Pt}}$ and $\Theta_{\mathrm{sH}}^{\mathrm{Pt}}$ as discussed in Sec. 2.4.

A proper characterization of all the parameters described above is necessary to accurately estimate the efficiency of generation and detection of spin currents, which is one of the key concerns of spintronics. The ultimate goal is to be able to make efficient spintronics devices at finite temperatures [127], where intrinsic scattering mechanisms play an important role. The earliest attempt [29] to include spin memory loss in determining $l_{\mathrm{sf}}$ and $\Theta_{\mathrm{sH}}$ for Pt at room temperature relied on estimates available at $4.2 \mathrm{~K}$ from magnetoresistance experiments [15]. The almost complete lack of information about how interface parameters might depend on temperature motivated the work that is presented here.

To quantitatively describe the magnetic and transport properties of transition metals requires taking into account their degenerate electronic structures. For the layered structures that form the backbone of spintronics, the most promising way to combine complex electronic structures with transport theory is to use scattering theory formulated either in terms of nonequilibrium Green's functions or wavefunction matching [55] that are equivalent in the linear response regime [65]. With few exceptions [116], the attempts that have been made to address interface properties have been based upon circuit theory whereby quantum mechanical transmission matrices form boundary conditions to match solutions of semiclassical Boltzmann or diffusion equations on either side of the interface. Such calculations have been used to calculate interface resistances [69-71, 128-130], mixing conductances [131-133], the spin-dependent transparency of FM|superconducting interfaces [134] and recently the SML [115]. In all of these applications, it is tacitly assumed that the interface properties are temperature independent. A priori it is not clear what the effects of temperature will be. It was found that the interface resistance could be increased $(\mathrm{Co} \mid \mathrm{Cu})$ or reduced $(\mathrm{Fe} \mid \mathrm{Cr})$ by interface disorder depending on the Fermi surfaces on either side of the interface [70, 128].

A simple and effective way of including temperature-induced lattice and spin disorder in the adiabatic approximation in first-principles scattering calculations has been recently demonstrated [46, 67]. Using the Landauer-Büttiker formalism, the resistivity can be extracted from calculations of the conductance as a function of the length $L$ of the scattering region [46, 72]. By calculating local spin currents from the scattering theory results, we can make direct contact with experiments interpreted using the Valet-Fert (VF) formalism that is expressed in terms of these same variables [9]. By focussing on local currents in Chapter 2, we showed how the interface effects that are always present in scattering calculations could be factored out and illustrated the approach with calculations of the room temperature spin-flip diffusion lengths of Pt and Py, the polarization of Py and the SHA for Pt. 
In this chapter, we extend the above approach to study the temperature dependence of the transport properties of $\mathrm{Co} \mid \mathrm{Pt}$ and Py|Pt interfaces. Because the interface and bulk parameters are inextricably coupled, we will first determine the bulk parameters for Pt $\left(\rho, l_{\mathrm{sf}}\right)$, Co and Py $\left(\rho, \beta\right.$ and $\left.l_{\mathrm{sf}}\right)$ before determining the three parameters used to characterize collinear spin transport through an interface $\left(R_{I}\right.$, $\gamma$ and $\delta$ ). Through this study, we are able to address how thermal disorder and magnetic ordering of Py vs Co influence these parameters. We also investigate how proximity induced magnetization in Pt influences the interface.

The plan of the chapter is as follows. In Sec. 4.2 we summarize the VF model extended to include the effect of SOC at interfaces (Sec. 4.2.1) and describe how it will be used to extract interface parameters (Sec. 4.2.2). In Sec. 4.3 we briefly summarize the first-principles scattering theory $[45,46]$ and give details of how fully relaxed $\mathrm{Co} \mid \mathrm{Pt}$ and $\mathrm{Py} \mid \mathrm{Pt}$ interface geometries are constructed, how temperature is incorporated in the adiabatic approximation, how the necessary atomic sphere potentials are calculated and how the length of the scattering system is determined. In Sec. 4.4, we determine the temperature dependence of the Pt, Co and Py bulk transport parameters (Sec. 4.4.1) and of the interface transport parameters for Co|Pt and Py|Pt interfaces (Sec. 4.4.2). To the best of our knowledge, this is the first attempt to evaluate the effect of thermal disorder on interfaces. Sec. 4.5 contains a discussion of the results presented in the previous section.

\subsection{Methods}

The original VF model parametrized interfaces in terms of the spin-dependent interface resistances $R_{\uparrow}$ and $R_{\downarrow}$. The model was extended by Fert and Lee [118] to include interface SOC in the form of a spin-flip interface resistance. It was reformulated in terms of the SML parameter $\delta$ by Baxter et al. for $\mathrm{NM} \mid \mathrm{NM}^{\prime}$ interfaces between two nonmagnetic metals [16], and by Eid et al. for NM|FM interfaces between nonmagnetic and ferromagnetic metals [83]. We summarize this generalized VF model and extract the boundary conditions for a geometrically sharp $\mathrm{NM} \mid \mathrm{FM}$ interface in the next subsection.

\subsubsection{Valet-Fert model}

Starting from the Boltzmann formalism, Valet and Fert derived the following equations to describe a spin current flowing along the $z$ direction perpendicular to the interface plane for an axially symmetric geometry

$$
\begin{aligned}
& \frac{\partial^{2} \mu_{s}}{\partial z^{2}}=\frac{\mu_{s}}{l_{\mathrm{sf}}^{2}} \\
& j_{\sigma}(z)=-\frac{1}{e \rho_{\sigma}} \frac{\partial \mu_{\sigma}}{\partial z}
\end{aligned}
$$




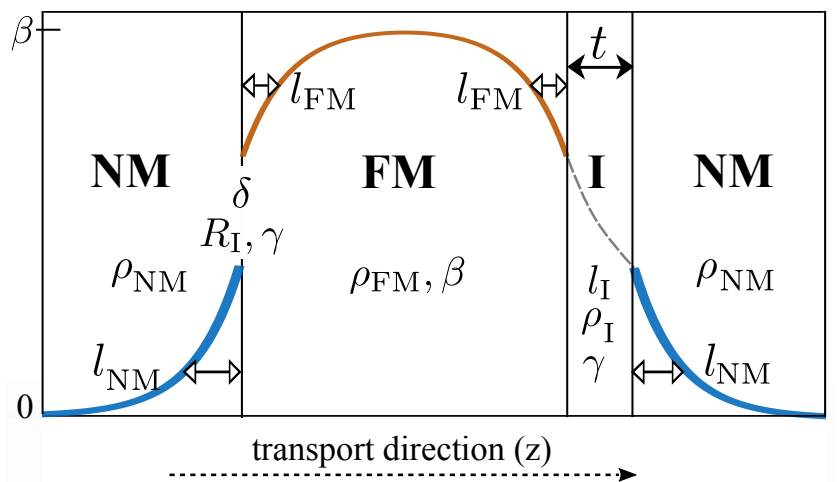

Figure 4.1: Normalized spin current $\widehat{j_{s}}(z)$ across a NM|FM|NM trilayer as described by the VF equations. $\widehat{j_{s}}(z)$ is assumed to be continuous at the FM|I and I|NM interfaces between the FM and NM bulk layers and the fictitious bulklike interface (I) layer with thickness $t$. In the limit that $t \rightarrow 0$, a discontinuity occurs in $\widehat{j_{s}}(z)$ at the left-hand NM|FM interface. .5

where $\sigma=\uparrow$ and $\downarrow$ for majority and minority spins, respectively, $\mu_{s}=\mu_{\uparrow}-\mu_{\downarrow}$ is the spin accumulation and $j_{s}=j_{\uparrow}-j_{\downarrow}$ is a pure spin current density. Equations (4.1a) and (4.1b) can be solved for $\mu_{\uparrow}, \mu_{\downarrow}, j_{\uparrow}$ and $j_{\downarrow}$ making use of the condition that the total current density $j=j_{\uparrow}+j_{\downarrow}$ is conserved in one-dimensional transport. The solutions are

$$
\begin{aligned}
& \mu_{s}(z)=A e^{z / l}+B e^{-z / l} \\
& \widehat{j_{s}}(z)=\beta-\frac{\left(1-\beta^{2}\right)}{2 e j \rho l}\left[A e^{z / l}-B e^{-z / l}\right]
\end{aligned}
$$

where $\widehat{j_{s}}(z) \equiv j_{s} / j$ is the normalized spin-current density and the spin-asymmetry (or polarization) parameter

$$
\beta=\frac{\rho_{\downarrow}-\rho_{\uparrow}}{\rho_{\downarrow}-\rho_{\uparrow}} .
$$

Instead of using the resistivity $\rho$ and polarization $\beta$, the spin-dependent resistivities $\rho_{\downarrow}$ and $\rho_{\uparrow}$ are frequently used with $\rho_{\uparrow}+\rho_{\downarrow}=4 \rho^{*}$ and $\rho_{\uparrow}-\rho_{\downarrow}=4 \rho^{*} \beta$ where the auxiliary quantity $\rho^{*}=\rho /\left(1-\beta^{2}\right)$.

The coefficients $A$ and $B$ in (4.2) are chosen to satisfy appropriate boundary conditions. This is illustrated in Fig. 4.1 with a sketch of $\widehat{j_{s}}(z)$ that arises in a diffusive symmetric $\mathrm{NM}|\mathrm{FM}| \mathrm{NM}$ trilayer when a current of electrons is passed from left to right. Far away (measured in units of $l_{\mathrm{NM}} \equiv l_{\mathrm{sf}}^{\mathrm{NM}}$ ) on the left, the spin current is unpolarized because of the symmetry of the two spin channels in a NM conductor; the solution (4.2) therefore only contains an exponentially increasing term. For a sufficiently thick FM (measured in units of $l_{\mathrm{FM}} \equiv l_{\mathrm{sf}}^{\mathrm{FM}}$ ) in which there 

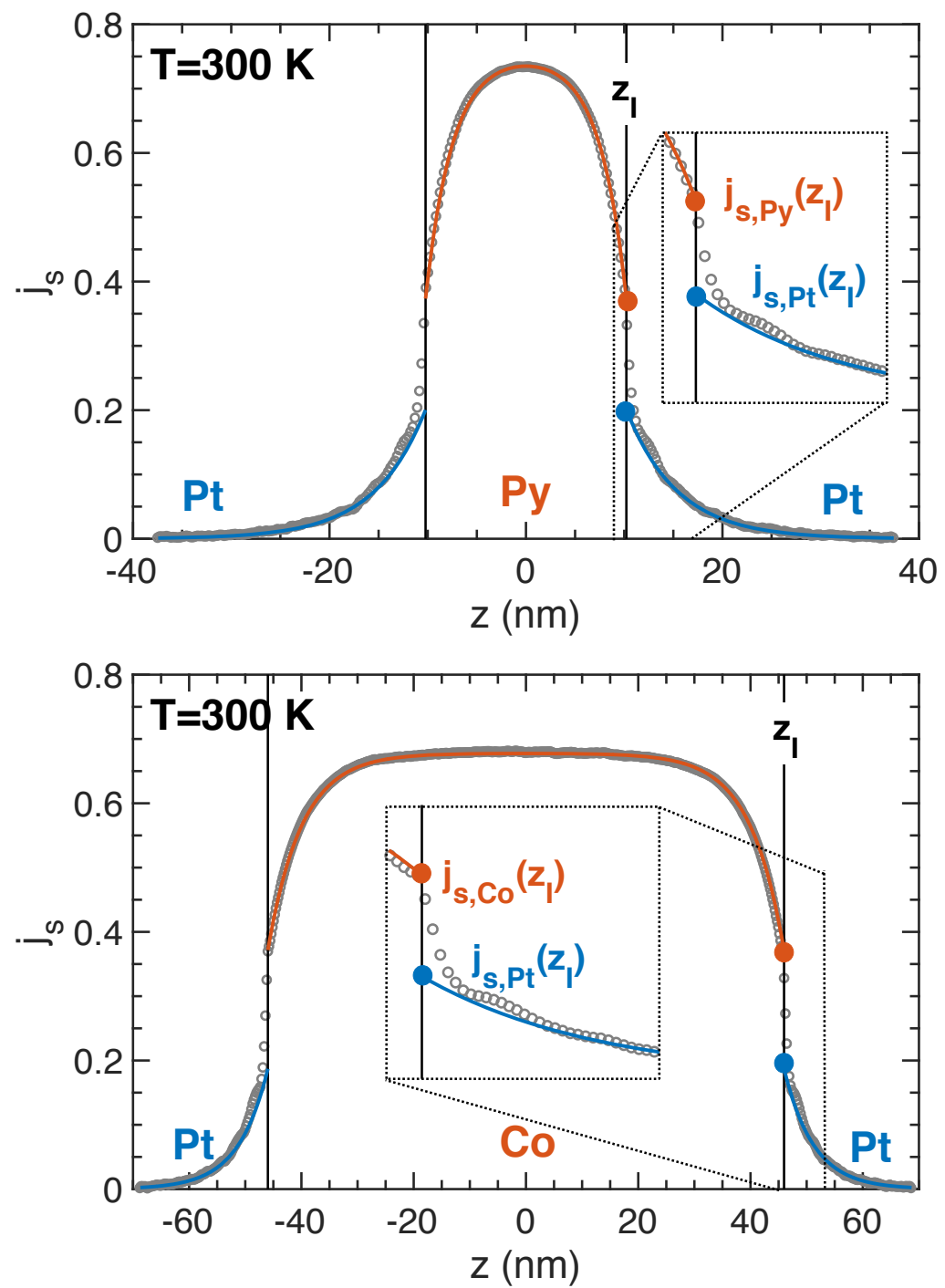

Figure 4.2: Spin current $\widehat{j_{s}}(z)$ calculated for (a) Pt|Py|Pt and (b) Pt $\mid$ Co|Pt trilayers at $300 \mathrm{~K}$ from the results of quantum mechanical scattering calculations. The data is fitted to the VF equations in bulk Pt (blue curves) and Py/Co (orange curve) and extrapolated to the interface $z_{\mathrm{I}}$ to obtain the values $\widehat{j}_{s, \mathrm{Pt}}\left(z_{\mathrm{I}}\right)$ for (a) and (b) separately and $\widehat{j}_{s, \text { Py }}\left(z_{\mathrm{I}}\right)$ and $\widehat{j}_{s, \mathrm{Co}}\left(z_{\mathrm{I}}\right)$, respectively, which are used to calculate $\delta$ and $\gamma$. 
is an asymmetry between the up-spin and down-spin channels, $\widehat{j_{s}}$ saturates to the value $\beta$. Far to the right in the NM material, it becomes zero again and (4.2) only contains an exponentially decreasing term. In the central FM material both terms are present. The spin and charge currents in the FM and NM bulk layers are characterised in terms of the resistivities $\rho$ and spin-flip diffusion lengths $l_{\mathrm{sf}}$. The asymmetry between the two spin channels in the FM is additionally characterised by the bulk spin asymmetry parameter $\beta$.

An interface is modelled by introducing a fictitious interface (I) layer with interface resistivity $\rho_{\mathrm{I}}$, polarization $\beta_{\mathrm{I}} \equiv \gamma$, and SDL $l_{\mathrm{I}} \equiv l_{\mathrm{sf}}^{\mathrm{I}}$. This is illustrated for the right-hand $\mathrm{FM} \mid \mathrm{NM}$ interface which is shown exploded as an FM|I|NM trilayer with a "bulk-like" I layer with finite thickness $t$. In this exploded representation, the spin current density is continuous at the FM|I and I|NM interfaces. For an interface area $A$, an interface resistance is defined as $A R_{\mathrm{I}}=\rho_{\mathrm{I}} t$ and the SML as $\delta=t / l_{\mathrm{I}}$. When $t \rightarrow 0$, a spin current discontinuity occurs at the interface as sketched for the left NM|FM interface in Fig. 4.1. This discontinuity is attributed to interface spin-flip scattering and described in terms of $\delta$. Instead of $A R_{\mathrm{I}}$ and $\gamma$ we can use the spin-dependent interface resistances $A R_{\downarrow}$ and $A R_{\uparrow}$ with $A R_{\uparrow}+A R_{\downarrow}=4 A R^{*}$, $A R_{\uparrow}-A R_{\downarrow}=4 A R^{*} \gamma$ and $A R_{\mathrm{I}}=A R_{\mathrm{I}}^{*}\left(1-\gamma^{2}\right)$.

As sketched in Fig. 4.1, interface spin-flipping is thus expected to lead to a discontinuity in $\widehat{j_{s}}(z)$. The result of calculating $\widehat{j_{s}}(z)$ from the output of quantum mechanical scattering calculations for (111) oriented diffusive $\mathrm{Pt}|\mathrm{Py}| \mathrm{Pt}$ and $\mathrm{Pt}|\mathrm{Co}| \mathrm{Pt}$ trilayers sandwiched between ballistic $\mathrm{Cu}$ leads is shown in Fig. 4.2 where each data point corresponds to a layer of atoms. The interface parameters cannot be determined simply from the calculated spin current by fitting because for the symmetric $\mathrm{NM}|\mathrm{FM}| \mathrm{NM}$ trilayer $\widehat{j_{s}}(z)$ depends not only on the three interface parameters but also on five bulk parameters: two parameters for bulk NM and three for bulk FM so $\widehat{j_{s}}(z) \equiv \widehat{j_{s}}\left(\rho_{\mathrm{NM}}, A R_{\mathrm{I}}, \rho_{\mathrm{FM}}, \beta_{\mathrm{I}}, \beta_{\mathrm{FM}}, l_{\mathrm{NM}}, \delta, l_{\mathrm{FM}}, z\right)$. Instead, we will first determine the bulk parameters with separate calculations for NM and FM materials. Then, using these parameters we will extrapolate $\widehat{j_{s}}(z)$ for the NM|FM|NM trilayer to the interface at $z=z_{\text {I }}$ from the NM side to yield $\widehat{j}_{s, \mathrm{NM}}\left(z_{\mathrm{I}}\right)$ and then from the FM side to yield $\widehat{j}_{s, \mathrm{FM}}\left(z_{\mathrm{I}}\right)$. This will leave us to determine three unknown interface parameters from two values of $\widehat{j_{s}}\left(z_{\mathrm{I}}\right) . A R_{\mathrm{I}}$ can be determined independently by calculating the resistance of a Pt|FM|Pt trilayer as a function of the thickness of the FM layer leaving us to determine $\delta$ and $\gamma$ from the discontinuity of $\widehat{j_{s}}(z)$ at the interface. In the following subsection, we will explain how this will be done without having to determine $\mu_{s}(z)$ explicitly.

\subsubsection{Interface discontinuity}

Viewing an interface as a fictitious bulk-like interface material transforms a $\mathrm{FM} \mid \mathrm{NM}$ bilayer of two materials into a FM|I|NM trilayer. The solutions (4.2) of the VF 
equations for the three distinct layers labelled $i=\mathrm{FM}, \mathrm{I}$, NM are

$$
\begin{aligned}
& \mu_{s i}(z)=A_{i} e^{z / l_{i}}+B_{i} e^{-z / l_{i}} \\
& j_{s i}(z)=\beta_{i}-\frac{\left(1-\beta_{i}^{2}\right)}{2 e j \rho_{i} l_{i}}\left[A_{i} e^{z / l_{i}}-B_{i} e^{-z / l_{i}}\right]
\end{aligned}
$$

and longitudinal spin transport is characterized by the nine transport parameters $\rho_{i}, \beta_{i}, l_{i}$ (with $\beta_{\mathrm{NM}} \equiv 0$ ). The over the normalized current will be omitted when it does not lead to any confusion.

We want to switch from an FM|I|NM picture where $\mu_{s}$ and $j_{s}$ are continuous everywhere to an FM|NM description with discontinuities in $\mu_{s}\left(z_{\mathrm{I}}\right)$ and $j_{s}\left(z_{\mathrm{I}}\right)$ at the sharp interface $z=z_{\text {I }}$ (Fig. 4.1). Continuity at the FM|I interface leads to

$$
\begin{aligned}
& \mu_{s, \mathrm{FM}}\left(z_{\mathrm{I}}\right)=A_{\mathrm{I}} e^{z_{\mathrm{I}} / l_{\mathrm{I}}}+B_{\mathrm{I}} e^{-z_{\mathrm{I}} / l_{\mathrm{I}}} \\
& j_{s, \mathrm{FM}}\left(z_{\mathrm{I}}\right)=\gamma-\frac{\left(1-\gamma^{2}\right)}{2 e j \rho_{\mathrm{I}} l_{\mathrm{I}}}\left[A_{\mathrm{I}} e^{z_{\mathrm{I}} / l_{\mathrm{I}}}-B_{\mathrm{I}} e^{-z_{\mathrm{I}} / l_{\mathrm{I}}}\right]
\end{aligned}
$$

and at the I|NM interface to

$$
\begin{aligned}
& \mu_{s, \mathrm{NM}}\left(z_{\mathrm{I}}+t\right)=A_{\mathrm{I}} e^{\left(z_{\mathrm{I}}+t\right) / l_{\mathrm{I}}}+B_{\mathrm{I}} e^{-\left(z_{\mathrm{I}}+t\right) / l_{\mathrm{I}}} \\
& j_{s, \mathrm{NM}}\left(z_{\mathrm{I}}+t\right)=\gamma-\frac{\left(1-\gamma^{2}\right)}{2 e j \rho_{\mathrm{I}} l_{\mathrm{I}}}\left[A_{\mathrm{I}} e^{\left(z_{\mathrm{I}}+t\right) / l_{\mathrm{I}}}-B_{\mathrm{I}} e^{-\left(z_{\mathrm{I}}+t\right) / l_{\mathrm{I}}}\right] .
\end{aligned}
$$

The coefficients $A_{\mathrm{I}}$ and $B_{\mathrm{I}}$ can be expressed in terms of $\mu_{s, \mathrm{FM}}\left(z_{\mathrm{I}}\right)$ and $\mu_{s, \mathrm{NM}}\left(z_{\mathrm{I}}+t\right)$. Taking the limit $t \rightarrow 0$ results in the expected discontinuity in $\mu_{s}$ and $j_{s}$ at the $\mathrm{FM} \mid \mathrm{NM}$ interface. Substituting $t / l_{\mathrm{I}}=\delta$ and $\rho_{\mathrm{I}}=A R_{\mathrm{I}} / t$ yields

$$
\begin{aligned}
& j_{s, \mathrm{FM}}\left(z_{\mathrm{I}}\right)=\gamma-\frac{\left(1-\gamma^{2}\right) \delta}{2 e j A R_{\mathrm{I}} \sinh \delta}\left[\mu_{s, \mathrm{NM}}\left(z_{\mathrm{I}}\right)-\mu_{s, \mathrm{FM}}\left(z_{\mathrm{I}}\right) \cosh \delta\right] \\
& j_{s, \mathrm{NM}}\left(z_{\mathrm{I}}\right)=\gamma-\frac{\left(1-\gamma^{2}\right) \delta}{2 e j A R_{\mathrm{I}} \sinh \delta}\left[\mu_{s, \mathrm{NM}}\left(z_{\mathrm{I}}\right) \cosh \delta-\mu_{s, \mathrm{FM}}\left(z_{\mathrm{I}}\right)\right]
\end{aligned}
$$

which is the desired result. In the next paragraph, we specialize to a symmetric $\mathrm{NM}|\mathrm{FM}| \mathrm{NM}$ trilayer and describe how we will extract the spin-flipping parameter $\delta$ and the spin-asymmetry parameter $\gamma$ for a FM|NM interface.

\section{Symmetric trilayer}

Although we are interested in the properties of a single interface between thermally disordered FM and NM, embedding an FM|NM bilayer between ballistic $\mathrm{NM}^{\prime}$ leads would result in a $\mathrm{NM}^{\prime}|\mathrm{FM}| \mathrm{NM} \mid \mathrm{NM}^{\prime}$ scattering geometry and a new $\mathrm{NM}^{\prime} \mid \mathrm{FM}$ interface with additional interface parameters. Instead, we consider a thermally 
disordered $\mathrm{NM}|\mathrm{FM}| \mathrm{NM}$ scattering region embedded between left and right ballistic $\left(\mathrm{NM}^{\prime}\right)$ leads. The advantages of this geometry are two-fold. (i) The inversion symmetry of the system makes $A_{\mathrm{FM}}=-B_{\mathrm{FM}}$. (ii) For sufficiently thick NM (and unpolarised leads), the spin currents far from the FM|NM interfaces decay to zero allowing us to assume $B_{\mathrm{NM}}($ left $)=A_{\mathrm{NM}}$ (right) $=0$. By choosing $z=0$ in the middle of the central FM layer, the expressions for $j_{s}(z)$ and $\mu_{s}(z)$ simplify to

$$
\begin{aligned}
& \mu_{s, \mathrm{FM}}(z)=A_{\mathrm{FM}}\left[e^{z / l_{\mathrm{FM}}}-e^{-z / l_{\mathrm{FM}}}\right] \\
& j_{s, \mathrm{FM}}(z)=\beta-\frac{\left(1-\beta^{2}\right)}{2 e j \rho_{\mathrm{FM}} l_{\mathrm{FM}}} A_{\mathrm{FM}}\left[e^{z / l_{\mathrm{FM}}}+e^{-z / l_{\mathrm{FM}}}\right] .
\end{aligned}
$$

Combining these to eliminate $A_{\mathrm{FM}}$ yields

$$
\mu_{s, \mathrm{FM}}(z)=2 e j \frac{\rho_{\mathrm{FM}} l_{\mathrm{FM}}}{1-\beta^{2}} \tanh \left(\frac{z}{l_{\mathrm{FM}}}\right)\left[\beta-j_{s, \mathrm{FM}}(z)\right] .
$$

In an analogous manner, the expressions for $j_{s}(z)$ and $\mu_{s}(z)$ in the right NM layer become

$$
\begin{aligned}
& \mu_{s, \mathrm{NM}}(z)=B_{\mathrm{NM}} e^{-z / l_{\mathrm{NM}}} \\
& j_{s, \mathrm{NM}}(z)=\frac{B_{\mathrm{NM}}}{2 e j \rho_{\mathrm{NM}} l_{\mathrm{NM}}} e^{-z / l_{\mathrm{NM}}}
\end{aligned}
$$

which when combined yield

$$
\mu_{s, \mathrm{NM}}(z)=2 e j \rho_{\mathrm{NM}} l_{\mathrm{NM}} j_{s, \mathrm{NM}}(z) .
$$

We can now replace $\mu_{s, \mathrm{FM}}\left(z_{\mathrm{I}}\right)$ and $\mu_{s, \mathrm{NM}}\left(z_{\mathrm{I}}\right)$ in equations (4.7) with $j_{s, \mathrm{FM}}\left(z_{\mathrm{I}}\right)$ and $j_{s, \mathrm{NM}}\left(z_{\mathrm{I}}\right)$. The choice of origin at the center of the FM layer means that $z_{\mathrm{I}}=L_{\mathrm{FM}} / 2$. Our final expressions for the values of the spin current at a FM|NM interface in terms of the transport parameters are

$$
\begin{aligned}
j_{s, \mathrm{FM}}\left(z_{\mathrm{I}}\right)= & \gamma-\frac{\left(1-\gamma^{2}\right) \delta}{A R_{\mathrm{I}} \sinh \delta} \times \\
& {\left[\rho_{\mathrm{NM}} l_{\mathrm{NM}} j_{s, \mathrm{NM}}\left(z_{\mathrm{I}}\right)-\cosh \delta \frac{\rho_{\mathrm{FM}} l_{\mathrm{FM}}}{1-\beta^{2}} \tanh \left(\frac{z_{\mathrm{I}}}{l_{\mathrm{FM}}}\right)\left\{\beta-j_{s, \mathrm{FM}}\left(z_{\mathrm{I}}\right)\right\}\right] } \\
j_{s, \mathrm{NM}}\left(z_{\mathrm{I}}\right)= & \gamma-\frac{\left(1-\gamma^{2}\right) \delta}{A R_{\mathrm{I}} \sinh \delta} \times \\
& {\left[\rho_{\mathrm{NM}} l_{\mathrm{NM}} j_{s, \mathrm{NM}}\left(z_{\mathrm{I}}\right) \cosh \delta-\frac{\rho_{\mathrm{FM}} l_{\mathrm{FM}}}{1-\beta^{2}} \tanh \left(\frac{z_{\mathrm{I}}}{l_{\mathrm{FM}}}\right)\left\{\beta-j_{s, \mathrm{FM}}\left(z_{\mathrm{I}}\right)\right\}\right] }
\end{aligned}
$$

Assuming that we know $\rho_{\mathrm{NM}}$ and $l_{\mathrm{NM}}$ for $\mathrm{NM}, \beta, \rho_{\mathrm{FM}}$, and $l_{\mathrm{FM}}$ for FM and $A R_{\mathrm{I}}$ for the interface, then using (4.12) we can determine $\gamma$ and $\delta$ if we know $j_{s, \mathrm{NM}}\left(z_{\mathrm{I}}\right)$ and $j_{s, \mathrm{FM}}\left(z_{\mathrm{I}}\right)$. 
Because of their implicit nature, (4.12) can only be solved numerically. We will need to determine the sensitivity of the solutions to uncertainities in all of the parameters as well as the extrapolated values $j_{s, \mathrm{FM}}\left(z_{\mathrm{I}}\right)$ and $j_{s, \mathrm{NM}}\left(z_{\mathrm{I}}\right)$. To identify the factors limiting the accuracy with which the parameters and spin-currents can be calculated, we need to recall some aspects of the scattering formalism used to calculate these quantities. This we do in the next section.

\subsection{Calculations}

Within the framework of density functional theory [43, 44], we solve the quantum mechanical scattering problem [120] for a general two terminal $\mathcal{L}|\mathcal{S}| \mathcal{R}$ configuration of a Pt|FM|Pt scattering region $(\mathcal{S})$ embedded between ballistic left $(\mathcal{L})$ and right $(\mathcal{R}) \mathrm{Cu}$ leads using a wave-function matching (WFM) method [47] implemented $[45,48]$ with a tight-binding (TB) muffin-tin orbital (MTO) basis [51, 74, $75]$ and generalized to include spin-orbit coupling and noncollinearity [46, 72] as well as temperature induced lattice and spin disorder $[66,67]$. The solution yields the scattering matrix $S$, from which we can directly calculate the conductance, as well as the full quantum mechanical wave function throughout the scattering region from which we can calculate position dependent charge and spin currents. The relevant spin current in this study is $j_{s z}^{z}(z)$ where the superscript indicates the direction of (charge or spin) transport and the subscript indicates the orientation of the spins which is here the magnetization direction $\widehat{\mathbf{m}}$ of Py, chosen to be parallel to the transport direction $z$. In this section, we discuss the considerations we make specifically for the $\mathrm{Pt}|\mathrm{Py}| \mathrm{Pt}$ and $\mathrm{Pt}|\mathrm{Co}| \mathrm{Pt}$ scattering region to extract reliable interface parameters.

\section{Supercells: Lattice mismatch}

To model various types of disorder, we assume periodicity in the directions transverse to the transport direction and construct periodic "lateral supercells" with which to model interfaces between fcc materials like Pt and Py that have different lattice parameters, $a_{\mathrm{Pt}}=3.923 \AA$ and $a_{\mathrm{Py}}=3.541 \AA$, respectively. Bulk Co is typically hcp below roughly $700 \mathrm{~K}$ [135]. However, when interfaced with a material like Pt, the Co thin films are predominantly fcc $[136,137]$. By preserving the volume of hcp Co with lattice parameters $a=2.507 \AA$ and $c=4.069 \AA$, we obtain an effective fcc lattice constant $a_{\mathrm{Co}}=3.539 \AA$. The similarity of this value to the lattice constant of Py means that Co and Py can be treated interchangeably when modelling the interface with Pt.

For a given orientation of an $\mathrm{A} \mid \mathrm{B}$ interface between materials $\mathrm{A}$ and $\mathrm{B}$, the periodicity of the atoms in the plane of the interface is described by primitive lattice vectors $\left\{\mathbf{a}_{1}, \mathbf{a}_{2}\right\}$ and $\left\{\mathbf{b}_{1}, \mathbf{b}_{2}\right\}$. We construct an ordered list of all A and B in-plane 

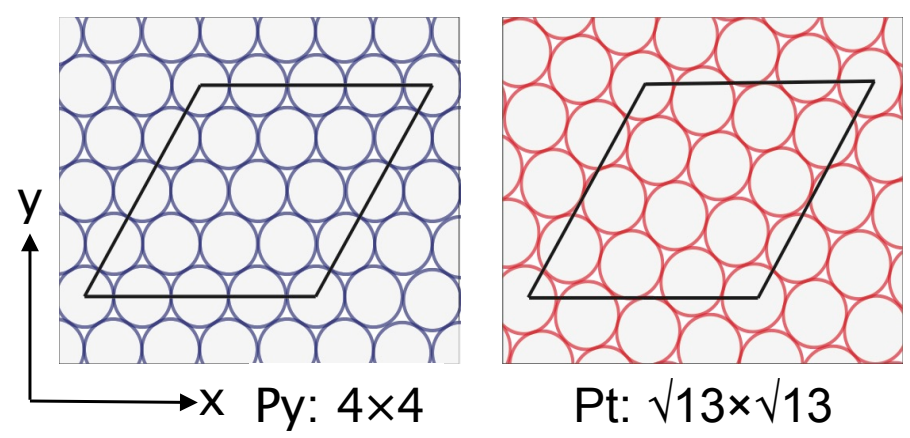

Figure 4.3: Two dimensional atomic planes of Py (left) and Pt (right) along fcc [111] direction. The parallelogram outlines the equivalent unit cells.

lattice vectors $\left|n_{1} \mathbf{a}_{1}+n_{2} \mathbf{a}_{2}\right|$, respectively $\left|m_{1} \mathbf{b}_{1}+m_{2} \mathbf{b}_{2}\right|$ and select a pair acceptably close in magnitude. In general, making these coincide will require rotating the two lattices with respect to one another as sketched in Fig. 4.3 where a $4 \times 4$ unit cell of (111) oriented Py (or Co) is matched to a $\sqrt{13} \times \sqrt{13}$ unit cell of similarly oriented Pt. These "superlattices" match to better than $0.1 \%$ and the residual mismatch is accommodated by uniformly expanding Pt. To ensure that the spin currents do not depend on the artificial lateral periodicity, the unit cells are doubled to $8 \times 8$ for FM and $2 \sqrt{13} \times 2 \sqrt{13}$ for Pt. These considerations are subject to the constraint that the computational expense of solving the scattering problem scales as the third power of the number of atoms in a lateral supercell.

The assumption of periodicity transverse to the transport direction allows us to make use of Bloch's theorem to label the wave functions that are solutions of the Schrödinger equation with a two dimensional (2D) wavevector. The corresponding 2D Brillouin zone (BZ) is sampled with $32 \times 32 k$-points for these supercells leading to equivalent samplings for $1 \times 1$ unit cells of Py and Pt of $256 \times 256$ and $\sim 230 \times 230$ respectively.

\section{Thermal disorder}

To carry out finite temperature calculations, we use a frozen thermal disorder scheme $[46,66,67]$ to displace atoms from their equilibrium positions and rotate magnetic moments from their equilibrium orientations. The distribution of the (uncorrelated) atomic displacements is assumed to be Gaussian and is characterized by a root-mean square displacement $\Delta$. For Pt, we choose a value of $\Delta$ to reproduce the experimental resistivity $[73,81]$ at any given temperature. For a given value of $\Delta$, multiple ( 10-20) random configurations of disorder are generated and all calculations are averaged over these configurations. For Py, $\Delta$ is derived from the Debye model [138] and spin disorder is modelled with a Gaussian distribution of rotations to reproduce the experimental magnetization [139] for a given temper- 
ature; this prescription has been shown to reproduce the experimentally observed resistivity very well $[46,67]$.

For Co, the spin disorder is modelled with a Gaussian distribution of rotations to reproduce the experimental magnetization [140] for a given temperature. Most experiments report the polycrystalline resisitivity profile for Co as a function of temperature [135]. Masumoto et al. [141] measured the experimental resistivity for single crystal hcp Co at $300 \mathrm{~K}$. We choose a value of $\Delta$ so that together with the spin disorder, the experimental resistivity at room temperature along [0001], $\rho_{\mathrm{Co}}=10.28 \mu \Omega \mathrm{cm}[141]$ is reproduced. Since there is no data available for monocrystalline Co at other temperatures and we use fcc instead of hcp Co, we use the Debye model and determine a Debye temperature $(450 \mathrm{~K})$ that yields the chosen $\Delta$ at $300 \mathrm{~K} . \Delta$ and the corresponding resistivities at all temperatures for fcc Co are then determined using a combination of Debye model with Debye temperature $450 \mathrm{~K}$ to describe the lattice disorder and spin disorder that reproduces the experimental magnetization.

Though the resistivity of bulk Pt can be calculated entirely from first principles within the adiabatic approximation by performing first-principles phonon calculations, populating the resulting phonon modes at a fixed temperature $T$, taking snapshots of the superimposed phonons, determining the resistance $R$ of various lengths $L$ of thermally disordered material and finally extracting $\rho$ from $R(L)$, the agreement with experiment, though good, is not perfect [67]. Spin disorder in magnetic materials can be treated analogously but additional approximations are necessary because spin-wave theory underestimates the magnetization decrease induced by temperature [67]. The tediousness of calculating phonon and magnon dispersion relations for magnetic alloys motivated us to adopt the simpler Gaussian disorder approach sketched above not only for Py but also for Pt and Co. Thus in the results we will present below, experimentally observed bulk resistivities are reproduced by construction.

\section{Potentials}

Bulk potentials for all atomic species $(\mathrm{Cu}, \mathrm{Pt}, \mathrm{Ni}, \mathrm{Fe}, \mathrm{Co})$ are calculated in the atomic spheres (AS) approximation (ASA) using the TB-LMTO method [51, 74, 75]. AS potentials for Ni and Fe are evaluated self-consistently for the fcc substitutional random alloy Py using the coherent potential approximation (CPA) [54] implemented with TB-MTOs [53].

In many experiments involving (Pd and) Pt, interface effects are expected to depend strongly on proximity-induced Pt magnetization [121]. Since we are focussing on the evaluation of interface parameters in this chapter, we will test this hypothesis by constructing interfaces with and without proximity-induced magnetism. In the simplest, default scenario, no magnetism is induced in Pt by proximity to FM. Because of the complexity of the FM|Pt interface and the inability of 


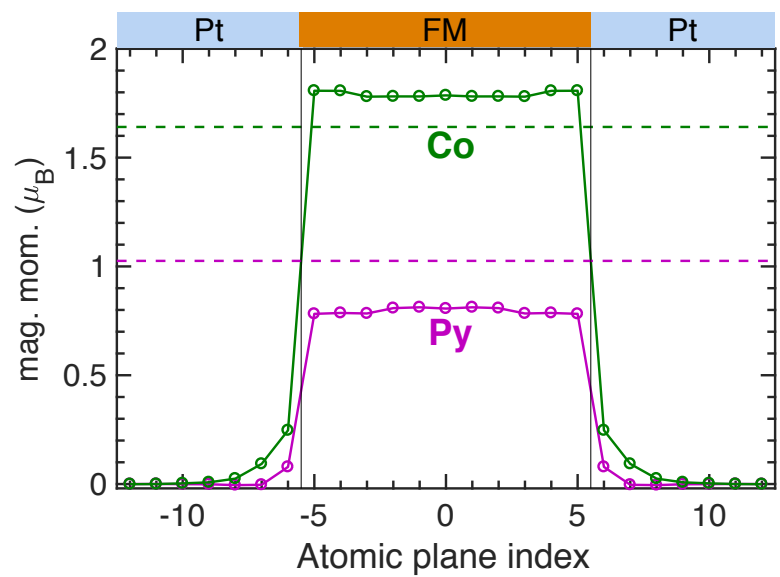

Figure 4.4: Magnetic moment profile of an fcc (111) oriented Pt|FM|Pt (FM=Py, Co) geometry calculated self-consistently using the coherent potential approximation. 11 layers of trigonally distorted pseudomorphic FM are sandwiched between semiinfinite Pt. The atomic moment is shown as a function of the plane number for Py (pink) and Co (green). For comparison, the horizontal dashed lines indicate the atomic moments calculated for bulk unstrained fcc Co (green) and Py (pink).

current CPA implementations to treat large unit cells, we study the magnetic moments induced in Pt by a putative FM grown pseudomorphically on "bulk" Pt; the in-plane lattice constant of a (111) oriented fcc FM is expanded to match that of Pt while the out-of-plane lattice constant is reduced to keep the volume of the FM unchanged.

The magnetization profiles of 11 atomic layers of pseudomorphic Py and Co sandwiched between cubic fcc Pt obtained with self-consistent CPA calculations are shown in Fig. 4.4. The magnetic moment induced in Pt by proximity to Py decreases rapidly from $0.08 \mu_{B}$ per Pt atom in the interface layer to $0.005 \mu_{B}$ (by 95\%) over 5 layers. The magnetic moment profile of Py is essentially constant at $0.8 \mu_{B}$ per atom with little change in the interface layer. For comparison, the average magnetic moment of bulk unstrained Py is $1 \mu_{B}$ so that the effect of the $10.7 \%$ strain is to reduce the Py moment.

Bulk fcc Co has a large magnetic moment of $1.64 \mu_{B}$. Stretching its in-plane lattice constant $a_{\mathrm{Co}}=3.55 \AA$ to make it match $a_{\mathrm{Pt}}$ while conserving its volume increases the moment to $1.78 \mu_{B}$. This stretched Co induces a moment of $0.25 \mu_{B}$ in the adjacent Pt layer. The induced moment decreases to $0.003 \mu_{B}$ (by 99\%) over 5 layers. Why strain affects the moments in Py and Co differently goes beyond the scope of this thesis.

In the scattering calculations to be discussed in the next section, we will replace the bulk non-magnetic Pt potentials for 7 layers of Pt next to the interface 
with magnetized Pt potentials and compare the resulting spin currents in the two cases. Since the Py moment is reduced by strain in the pseudomorphic structure and this may result in an underestimation of the moments induced in Pt, we will use the spin polarized Pt potentials determined for $\mathrm{Pt}|\mathrm{Co}| \mathrm{Pt}$ as input to the scattering calculations for both Py|Pt and Co|Pt interfaces.

To determine the spin-flip diffusion length, we will in the next section inject a fully spin-polarized current $\left(\left|j_{s}\right|=1\right)$ into the scattering region from "half-metallic ferromagnetic" (HMF) Cu leads denoted $\mathrm{Cu} \uparrow$ or $\mathrm{Cu} \downarrow$ as demonstrated in Sec. 2.3.1. These artificial leads are constructed by adding a constant repulsive term to the AS potential of the minority (majority) spin states so that these states are lifted above the Fermi level and the spin-current density consequently consists of only majority (minority) spin states.

\section{Slab length}

Eqs. (4.12) include an apparent dependence on the slab length $z_{\mathrm{I}}=L_{\mathrm{FM}} / 2$. While it is expected that the spin current must saturate to $\beta$ at the center of a sufficiently long FM slab, it is not a priori clear how the spin current close to the interface depends on the slab length. It turns out that for $L_{\mathrm{FM}} \geq 6 l_{\mathrm{FM}}$, the spin current close to the FM|NM interface is independent of $L_{\mathrm{FM}}$. Both left and right slabs of Pt have $L_{\mathrm{Pt}}>4 l_{\mathrm{Pt}}$ ) to ensure any spin current in $\mathrm{Pt}$ has decayed to a negligible value close to the leads.

\subsection{Results and Discussion}

The bulk transport properties of Pt, Py and Co are calculated for the temperature range $100-500 \mathrm{~K}$ in Sec. 4.4.1 allowing $j_{s, \mathrm{FM}}\left(z_{\mathrm{I}}\right)$ and $j_{s, \mathrm{Pt}}\left(z_{\mathrm{I}}\right)$ to be determined. In Sec. 4.4.2 we calculate the interface resistance $A R_{\mathrm{I}}$ for an $\mathrm{FM} \mid \mathrm{Pt}$ interface using the Landauer-Büttiker formalism. The remaining two parameters $\gamma$ and $\delta$ are determined by solving equations (4.12). No calculations were carried out at $100 \mathrm{~K}$ for bulk Co or Pt $|\mathrm{Co}| \mathrm{Pt}$ because $l_{\mathrm{sf}}^{\mathrm{Co}}$ was estimated to be $\geq 25 \mathrm{~nm}$ which when combined

with the calculated value of $l_{\mathrm{sf}}^{\mathrm{Pt}}=22 \mathrm{~nm}$ would require constructing a trilayer with more than 100,000 atoms and require excessive computational resources.

\subsubsection{Bulk materials}

The bulk resistivities $\rho_{\mathrm{Pt}}, \rho_{\mathrm{Py}}$ and $\rho_{\mathrm{Co}}$ can be determined directly from the scattering matrix using the Landauer-Büttiker formalism. The transport polarization $\beta$ for Py and Co as well as the spin-flip diffusion lengths $l_{\mathrm{Pt}}, l_{\mathrm{Py}}$ and $l_{\mathrm{Co}}$ are extracted from calculations of the spin current $j_{s}(z)$. Using these bulk parameters we fit the $j_{s}(z)$ calculated for Pt|Py|Pt and Pt|Co|Pt trilayers shown in Fig. 4.2(a) and Fig. 4.2(b) respectively. Fitting $j_{s}(z)$ in the FM material yields the orange curve in Fig. 4.2 and 


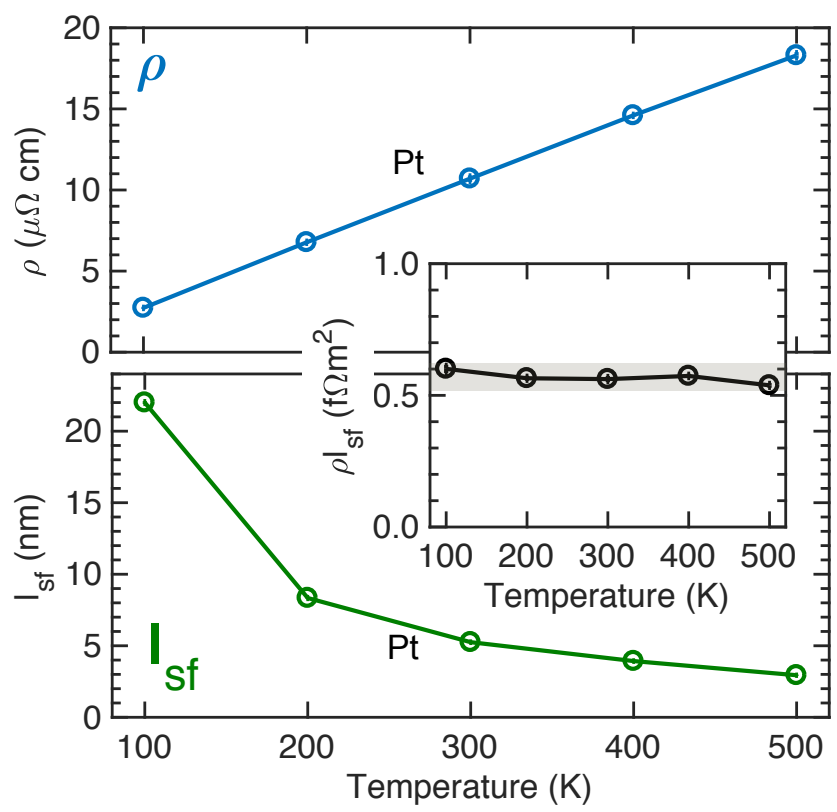

Figure 4.5: Resistivity $\rho_{\mathrm{Pt}}$ and spin-flip diffusion length $l_{\mathrm{Pt}}$ as a function of temperature for bulk Pt. Inset: Constant $\rho_{\mathrm{Pt}} l_{\mathrm{Pt}}$ within the shaded grey area as a function of temperature.

the value of $A_{\mathrm{FM}}$ using (4.8b). Fitting $j_{s}(z)$ in Pt yields the blue curve in Fig. 4.2 and the value of $B_{\mathrm{Pt}}$ using (4.10b). This allows us to calculate $j_{s, \mathrm{FM}}\left(z_{\mathrm{I}}\right)$ and $j_{s, \mathrm{Py}}\left(z_{\mathrm{I}}\right)$ by extrapolation to the interface.

\section{Resistivity}

By construction, the Gaussian lattice and spin disorder we use reproduces the experimentally observed [73, 81] resistivities of bulk Pt (Fig. 4.5) and Py (Fig. 4.6) at all temperatures. For Co, the lattice and spin disorder chosen at $300 \mathrm{~K}$ as described in Sec. 4.3 reproduce the experimental resistivity value of $10.28 \mu \Omega \mathrm{cm}$ observed for crystalline hcp Co [141]. Using the same lattice and spin disorder for an fcc structure yields a resistivity of $9.56 \pm 0.08 \mu \Omega \mathrm{cm}$ at $300 \mathrm{~K}$. Co fcc resistivities at other temperatures are calculated as described in Sec. 4.3 and are plotted in Fig. 4.6. Here, it is important to note how the resistivity behaves for Py and Co to better anticipate the behaviour of the remaining spin transport parameters. The chemi$\mathrm{cal}$, alloy disorder in Py leads to a finite value of $\rho \sim 4 \mu \Omega \mathrm{cm}$ at $0 \mathrm{~K}$ whereas the resistivity of ordered Co approaches zero. A Curie temperature of $872 \mathrm{~K}$ for Py versus $1385 \mathrm{~K}$ for Co implies that at any given temperature the magnetic ordering in Co is stronger. The combination of chemical and magnetic disorder in Py makes its resistivity change more rapidly as a function of temperature. 

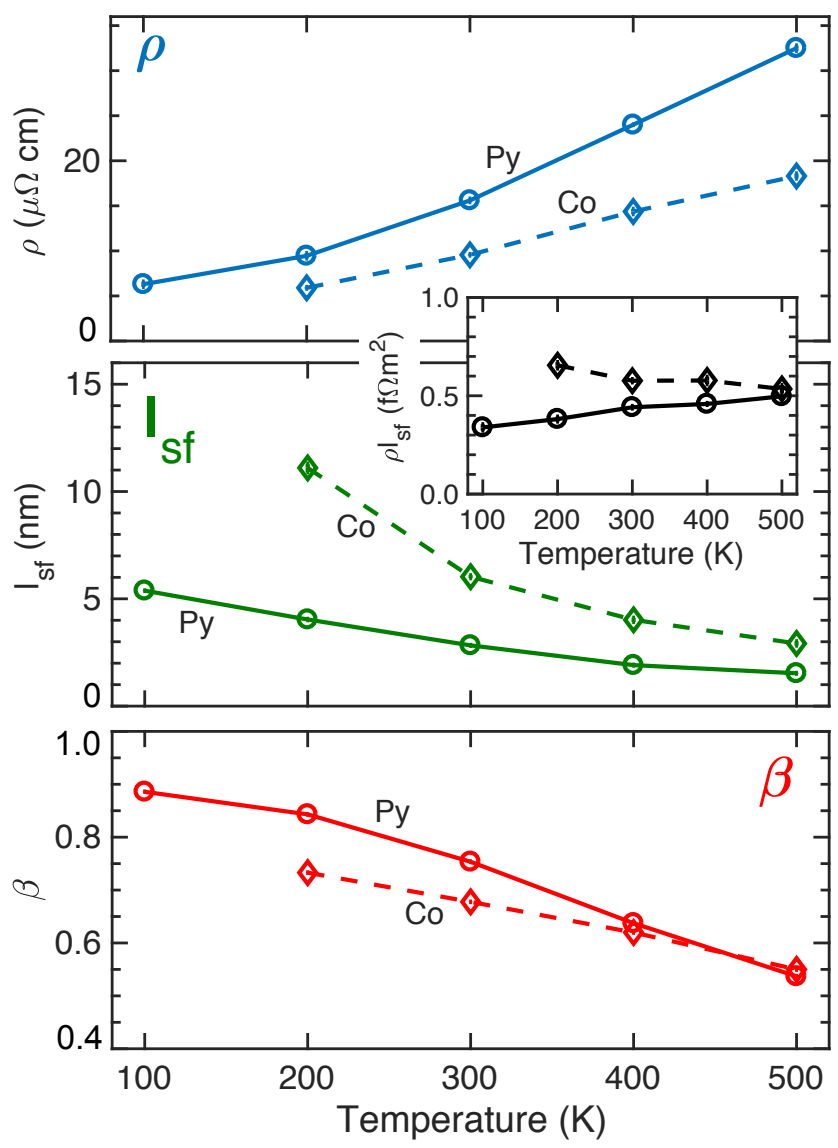

Figure 4.6: Spin asymmetry parameter $\beta$, spin-flip diffusion length $l_{\text {sf }}$ and resistivity $\rho$ as a function of temperature for bulk Py (circles) and bulk Co (diamonds). Inset: $\rho l_{\text {sf }}$ for bulk Py (circles) and bulk Co (diamonds) as a function of temperature.

All other parameters that we report have been calculated using the same thermal disorder employed for the resistivity calculations. We did not attempt to reproduce the resistivities reported for thin films that differ from the known bulk values because so little is known about the microscopic disorder (impurities, vacancies, self interstitials, grain boundaries, surfaces etc.) that might give rise to the differences.

\section{Spin-flip diffusion length $l_{\mathrm{sf}}: \mathrm{Pt}$}

A fully polarized spin current $j_{s}(0)=1$ injected into thermally disordered Pt decays exponentially to zero, $j_{s}(z)=C \exp (-z / l)$. Using the procedure described in Sec. 2.3.1 for a Au个|Pt|Au scattering geometry, we obtain $l_{\mathrm{Pt}}$ from spin current cal- 


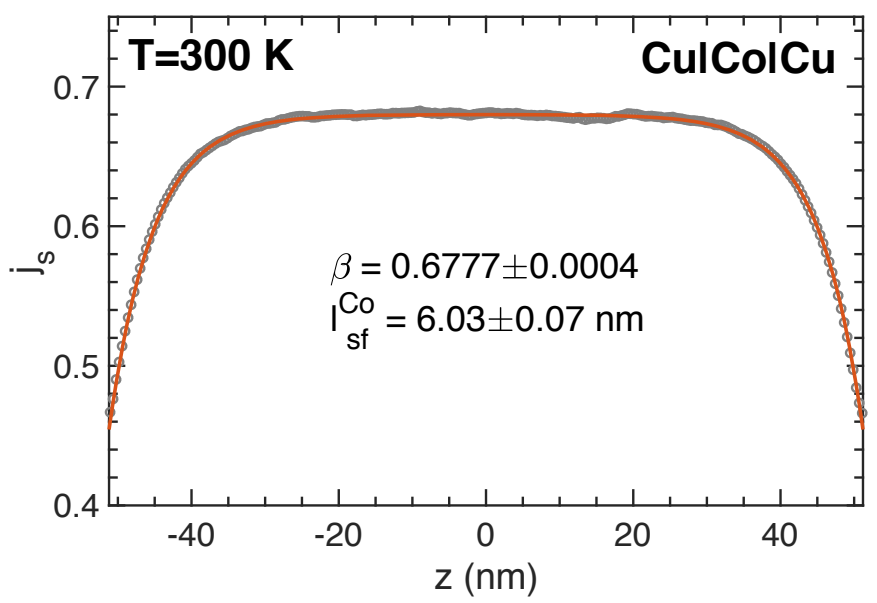

Figure 4.7: An unpolarized charge current enters a sufficiently long slab of an $8 \times 8$ lateral supercell of Co at $300 \mathrm{~K}$. The grey circles show the resulting spin current $j_{s}$ polarized along the magnetization direction $\hat{z}$. The orange curve shows the fit for $j_{s}$ that yields $\beta=0.6777 \pm 0.0004$ and $l_{\mathrm{sf}}=6.03 \pm 0.07 \mathrm{~nm}$.

culations for temperatures between 100-500 K. The results for $l_{\mathrm{Pt}}$ shown in Fig. 4.5 exhibit a $1 / \mathrm{T}$ dependence and satisfy the relationship $\rho_{\mathrm{Pt}} l_{\mathrm{Pt}}=0.57 \pm 0.05 \mathrm{f} \Omega \mathrm{m}^{2}$ in accordance with the Elliott-Yafet mechanism [13, 14], as shown in Fig. 4.5 (inset).

\section{$l_{\mathrm{sf}}$ and $\beta$ : Py and Co}

A charge current passed through a magnetic material is naturally polarized along the magnetization direction. The way in which it approaches its equilibrium polarization value $\beta$ is described in equation (4.8b) by the spin-flip diffusion length $l_{\mathrm{FM}}$. We studied the computational aspects of extracting $\beta$ and $l_{\text {Py }}$ from $j_{s}(z)$ calculated for Py at $300 \mathrm{~K}$ in Secs. 2.3.2 and 2.3.3. Here we show the $z$ dependence of the spin current calculated for a symmetric $\mathrm{Cu}|\mathrm{Co}| \mathrm{Cu}$ scattering geometry with room temperature thermal disorder in Fig. 4.7. By fitting $j_{s}(z)$ to (4.8b), we extract values of $\beta=0.68$ and $l_{\mathrm{Co}}=6.03 \mathrm{~nm}$ for room temperature Co.

The temperature dependence of $l_{\mathrm{FM}}$ and $\beta$ is plotted in Fig. 4.6 for both Py and Co. For Py, $l_{\mathrm{Py}}$ decreases from $5.4 \mathrm{~nm}$ at $100 \mathrm{~K}$ to $1.5 \mathrm{~nm}$ at $500 \mathrm{~K}$. Co has a much larger $l_{\mathrm{Co}}=11.1 \mathrm{~nm}$ at $200 \mathrm{~K}$ that decreases to $2.9 \mathrm{~nm}$ at $500 \mathrm{~K}$. The relatively smaller values of $l_{\mathrm{Py}}$ can be attributed to the chemical disorder that is present at all temperatures in addition to the greater thermal spin disorder. Unlike Pt that conforms to the Elliot-Yafet model behaviour, the product $\rho l_{\mathrm{sf}}$ is not a constant at all temperatures for Py and Co, as shown in Fig. 4.6 (inset). $\rho l_{\text {sf }}$ increases with temperature for Py, but decreases for Co. The interplay of thermal (lattice and spin) and chemical disorder makes it difficult at this point to systematically explain 
our findings.

Polarization $\beta$ is the difference between majority and minority resistivities. For Py at $0 \mathrm{~K}$, the minority resistivity $\rho_{\min }$ is $\sim 200$ times larger than the majority resistivity $\rho_{\text {maj }}$ [46]. This large $\beta \sim 0.99$ decreases to still large values of 0.88 at $100 \mathrm{~K}$, 0.75 at RT and 0.54 at $500 \mathrm{~K}$. For Co, the polarization is relatively smaller.

\subsubsection{Interfaces}

\section{Interface resistance}

We calculate the conductance $G(L)$ of a scattering region of length $L$ using the Landauer-Büttiker formalism [120] whereby $G$ is expressed in terms of the probability that Bloch states in the left lead $\mathcal{L}$ attached to the scattering region $\mathcal{S}$ are transmitted through the scattering region into the right lead $\mathcal{R}$. The results of doing this for a length $L$ of thermally disordered Pt sandwiched between ballistic $\mathrm{Cu}$ leads are shown in Fig. 4.8 for $T=300 \mathrm{~K}$. The resistivity is extracted as a slope of the linear fit of $R(L)=1 / G(L)$ plotted as a function of $L[46,66,72]$. For $L=0$, the resistance does not vanish; there is a finite intercept because a finite cross section $A$ of a ballistic material has a finite conductance, the Sharvin conductance $G_{\mathrm{Sh}}$ [142] . In addition, there is a resistance $R_{\mathrm{Cu} \mid \mathrm{Pt}}$ associated with each interface so the total resistance consists of

$$
A R\left(L_{\mathrm{Pt}}\right)=\rho_{\mathrm{Pt}} L_{\mathrm{Pt}}+2 A R_{\mathrm{Cu} \mid \mathrm{Pt}}+1 / G_{\mathrm{Sh}} .
$$

This interface resistance, however, is for an interface between a ballistic $(T=0)$ $\mathrm{Cu}$ lead and diffusive Pt. To determine the interface resistance $A R_{\mathrm{I}}$ between two diffusive interfaces requires more work.

We extract $A R_{\mathrm{I}}$ in a two step procedure. We calculate the total resistance for a symmetric Pt|FM|Pt trilayer embedded between ballistic leads for a variable length $L_{\mathrm{FM}}$ of FM and fixed length $L_{\mathrm{Pt}}$ of Pt. Both $L_{\mathrm{FM}}$ and $L_{\mathrm{Pt}}$ should be much longer than the respective mean free paths so that the total areal resistance for the scattering region can be expressed in terms of a series resistor model as

$$
\begin{aligned}
A R\left(L_{\mathrm{FM}}\right) & =\rho_{\mathrm{FM}} L_{\mathrm{FM}}+\rho_{\mathrm{Pt}} L_{\mathrm{Pt}} \\
& +2 A R_{\mathrm{FM} \mid \mathrm{Pt}}+2 A R_{\mathrm{Pt} \mid \text { lead }}+1 / G_{\mathrm{Sh}}
\end{aligned}
$$

Here, $R_{\mathrm{FM} \mid \mathrm{Pt}}$ is the interface resistance $A R_{\mathrm{I}}$ we are interested in, $R_{\mathrm{Pt} \mid \text { lead }}$ is the interface resistance between Pt and the ballistic lead, and $G_{\mathrm{Sh}}$ is the Sharvin conductance of the lead. Noting that

$$
A R\left(L_{\mathrm{FM}}=0\right)=\rho_{\mathrm{Pt}} L_{\mathrm{Pt}}+2 A R_{\mathrm{Pt} \mid \text { lead }}+1 / G_{\mathrm{Sh}}
$$

we can subtract (4.15) from (4.14) to obtain

$$
A\left[R\left(L_{\mathrm{FM}}\right)-R\left(L_{\mathrm{FM}}=0\right)\right]=\rho_{\mathrm{FM}} L_{\mathrm{FM}}+2 A R_{\mathrm{FM} \mid \mathrm{Pt}}
$$




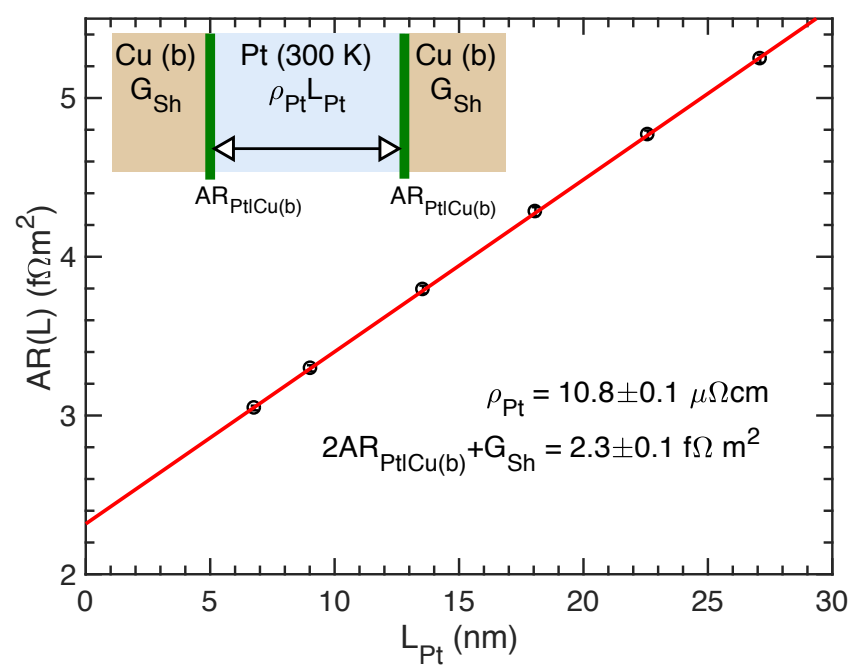

Figure 4.8: Total resistance for diffusive Pt sandwiched between ballistic $\mathrm{Cu}$ leads as a function of the Pt thickness $L_{\mathrm{Pt}}$. A linear fit $R(L)$ yields $\rho_{\mathrm{Pt}}$ as the slope; the intercept is a sum of interface and Sharvin contributions.

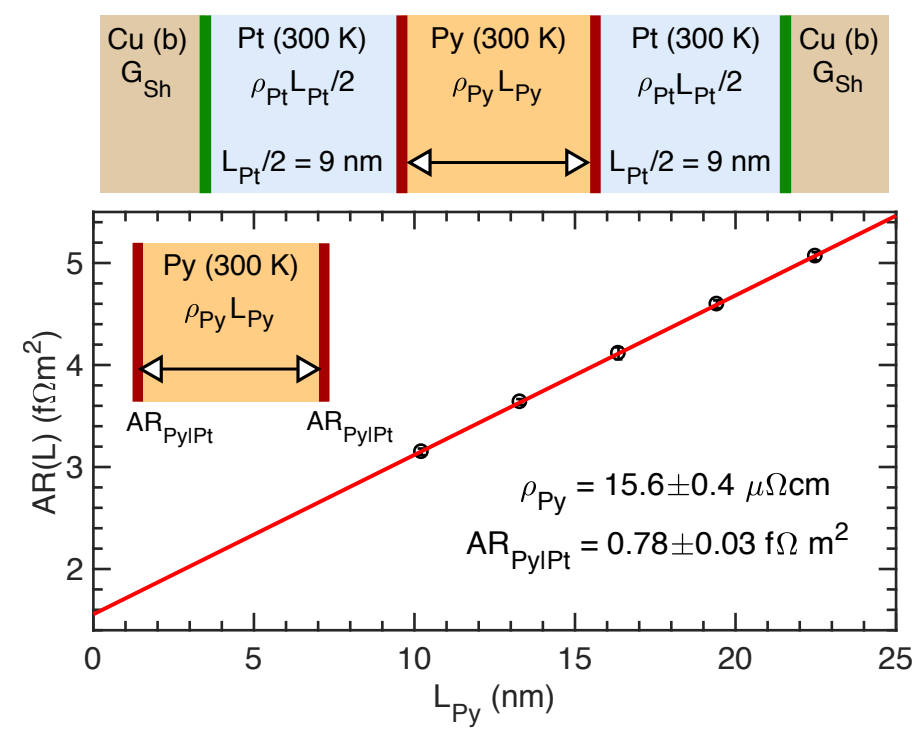

Figure 4.9: Total resistance of a diffusive $\mathrm{Pt}|\mathrm{Py}| \mathrm{Pt}$ trilayer sandwiched between $\mathrm{Cu}$ leads as a function of the Py thickness $L_{\mathrm{Py}}$ for a fixed Pt thickness $L_{\mathrm{Pt}}=18 \mathrm{~nm}$. To extract $\rho_{\text {Py }}$ and $A R_{\mathrm{I}}$, resistances for $\mathrm{Pt}\left|\mathrm{Py}\left(L_{\mathrm{Py}}\right)\right| \mathrm{Pt}$ are calculated and all contributions from $\mathrm{Cu}$ and $\mathrm{Pt}$ are subtracted leaving $A R_{\mathrm{I}}$ as the intercept and $\rho_{\mathrm{Py}}$ as the slope for the linear fit. 


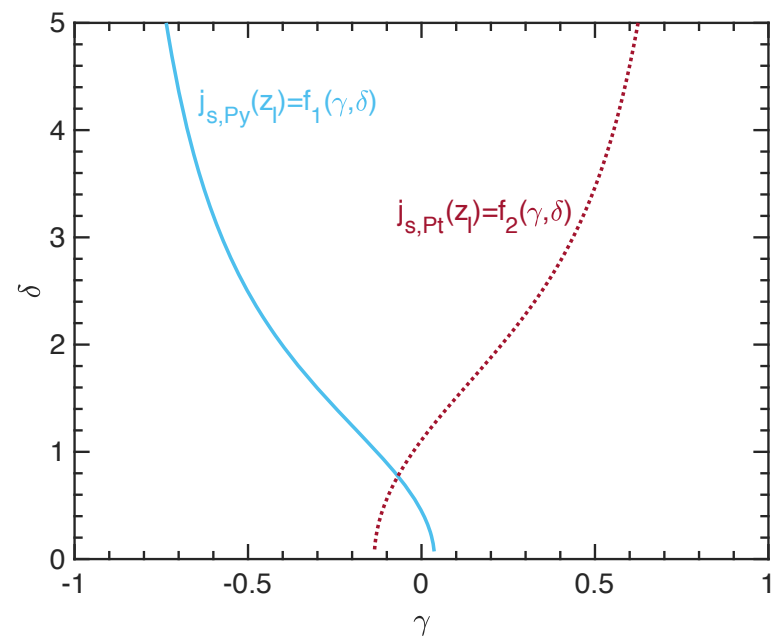

Figure 4.10: Graphical representation of numerical solution for $\gamma$ and $\delta$ obtained by solving Eqs. 4.12 at boundary values $j_{s, \mathrm{Py}}\left(z_{\mathrm{I}}\right)$ and $j_{s, \mathrm{Pt}}\left(z_{\mathrm{I}}\right)$.

and observe that $A R_{\mathrm{FM} \mid \mathrm{Pt}}$ can be calculated as the intercept of the above resistance difference determined as a function of $L_{\mathrm{FM}}$. The second step (4.15) requires calculating the total resistance of a length $L_{\mathrm{Pt}}$ of diffusive Pt sandwiched between the same leads used in the first step. The result of doing this for RT Py|Pt is shown in Fig. 4.9. It can be seen that an interface resistance can be determined with an acceptably small uncertainty, $A R_{\mathrm{I}}=0.78 \pm 0.01 \mathrm{f} \Omega \mathrm{m}^{2}$. For RT Co|Pt $A R_{\mathrm{I}}$ is somewhat larger, $0.83 \pm 0.03 \mathrm{f} \Omega \mathrm{m}^{2}$.

The temperature dependence of the interface resistance is shown in the top panel of Fig. 4.11. $A R_{\mathrm{I}}$ is seen to decrease monotonically with temperature for both Py|Pt and Co|Pt. Microscopically, the total transmission through the $\mathrm{FM} \mid \mathrm{Pt}$ interface increases with increasing thermal disorder. Since the magnetic ordering in Py is weaker than in Co, the decrease in $A R_{\mathrm{I}}$ with temperature is more rapid for Py|Pt.

\section{Spin memory loss and interface spin-asymmetry $\gamma$}

Now that we know all of the variables besides $\delta$ and $\gamma$ in (4.12) for both Py|Pt and $\mathrm{Co} \mid \mathrm{Pt}$, we can solve the two equations simultaneously to yield the remaining two unknown interface parameters. We illustrate this procedure for the RT Py|Pt case by substituting the five bulk parameters for Py and Pt as well as the Py|Pt interface resistance we have just calculated together with the values of $j_{s, \mathrm{Pt}}\left(z_{I}\right)$ and $j_{s, \mathrm{Py}}\left(z_{I}\right)$ into (4.12). The equations are graphically represented as contours in $(\gamma, \delta)$ space in Fig. 4.10 and the solutions found using standard root-searching algorithms. The single crossing indicates that there exists a unique solution for these parameters. 


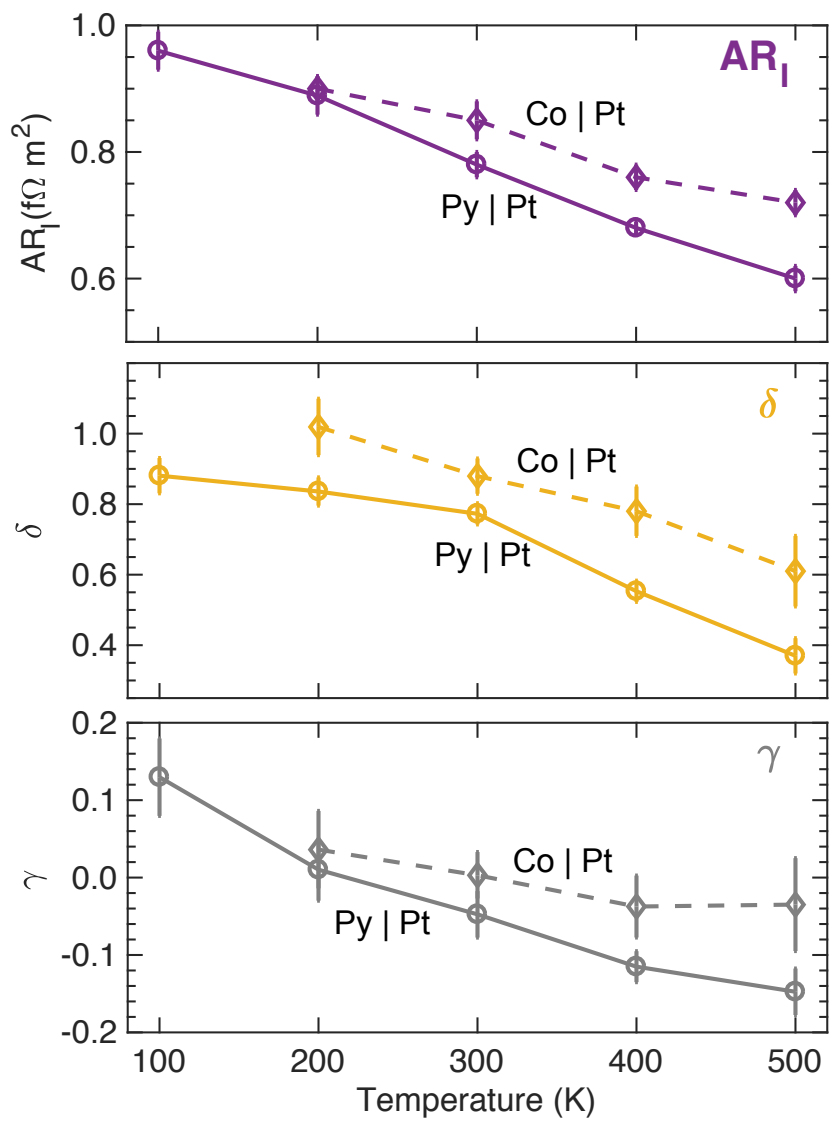

Figure 4.11: Interface parameters $A R_{\mathrm{I}}, \delta$ and $\gamma$ for Py|Pt (circles, solid lines) and $\mathrm{Co} \mid \mathrm{Pt}$ (diamonds, dashed lines) interfaces plotted as a function of temperature.

The topology of the crossing indicates the robustness of the solution set.

The temperature dependence of $\delta$ and $\gamma$ is plotted in Fig. 4.11. The error bars on these parameters are determined as follows: All input parameters, the five bulk parameters for FM and Pt, $R_{\mathrm{I}}$ and the values of $j_{s, \mathrm{Pt}}\left(z_{I}\right)$ and $j_{s, \mathrm{FM}}\left(z_{I}\right)$ span a finite range described by their error bars. Solutions for $\delta$ and $\gamma$ are extracted by substituting all possible combinations of the input parameters into (4.12). The range of $\delta$ and $\gamma$ determined from this exercise yields the error bars shown in the figures.

Like $R_{\mathrm{I}}, \delta$ and $\gamma$ decrease monotonically with temperature for both interfaces. $\gamma$ is found to vary in a small range between -0.1 and 0.1 for Py|Pt and between -0.03 and 0.03 for $\mathrm{Co} \mid \mathrm{Pt}$, displaying an at best weak dependence on its bulk counterpart $\beta$ in Py and Co. However, $\delta$, the main focus of our interest, shows a significant dependence on temperature and choice of FM. For both interfaces, it decreases monotonically with temperature. Its magnitude is larger for Co|Pt compared to Py|Pt for all temperatures in the range 200-500 K. At high temperatures, Co disor- 
ders more slowly than Py because of its higher Curie temperature, the interface is more abrupt and $\delta$ is higher. We expect the same to hold true at lower temperatures where the interface involving Co becomes more abrupt than that involving Py as Co orders completely and SOC-induced interface splittings are not washed out by alloy disorder. Like $R_{\mathrm{I}}$, the decrease in $\delta$ for Py|Pt is more rapid than for $\mathrm{Co} \mid \mathrm{Pt}, \delta$ going from 0.88 at $100 \mathrm{~K}$ to 0.37 at $500 \mathrm{~K}$ (a $58 \%$ decrease or $\sim 15 \%$ per $100 \mathrm{~K}$ ) for Py|Pt and from 1.02 at $200 \mathrm{~K}$ to 0.61 at $500 \mathrm{~K}$ (a $40 \%$ decrease or $\sim 13 \%$ per $100 \mathrm{~K})$ for Co|Pt.

Our results for $R_{\mathrm{I}}$ and $\delta$ show how a combination of alloy and spin disorder determine how charge and spin currents are transmitted through these interfaces. Transmission is facilitated by increasing bulk disorder. However, it must be kept in mind that underlying these parameters is the electronic structure mismatch between the two materials making up the interface and the strong spin-orbit coupling in Pt both of which depend only weakly on temperature. This explains why even at $500 \mathrm{~K}$ well below the Curie temperature of either Py or Co, the interface parameters are not even close to zero. Thus, the common assumption made in interpreting experiments of transparent $\mathrm{FM} \mid \mathrm{Pt}$ interfaces is not supported by our calculations. We will discuss our results in regard to experiment in Sec. 4.5.

\section{Effect of proximity induced magnetization in Pt}

We have found a significant spin-memory loss for all temperatures studied. So far, however, the proximity-induced magnetization of Pt has not been taken into account. The proposal that Pt magnetization plays a key role in determining the transport of spins through $\mathrm{FM} \mid \mathrm{NM}$ interfaces $[121,143]$ then poses the question as to how it might affect the interface parameters we have calculated. To address this question, we repeat the RT calculations for both Py|Pt and Co|Pt interfaces to determine the interface resistance $A R_{\mathrm{I}}$ and the Pt|FM|Pt trilayer spin current profile replacing "bulk, non-magnetic" Pt potentials adjacent to the FM interface with spin-polarized potentials. As discussed in Sec. 4.3, these are obtained from a CPA calculation for a pseudomorphic Pt|Co|Pt trilayer. Within the errorbar of the calculations, the values of $A R_{\mathrm{I}}$ are unchanged. In Fig. 4.12 we compare the spin current $j_{s}(z)$ close to the FM|Pt interface obtained without (blue circles) and with (red squares) magnetic moments induced in Pt for Py|Pt (upper) and $\mathrm{Co} \mid \mathrm{Pt}$ (lower). We see virtually no change in the spin currents and within the errorbars of the calculation no change in the discontinuity of $j_{s}(z)$ at the interface; thus $\delta$ and $\gamma$ are not affected by proximity induced magnetism in Pt. 

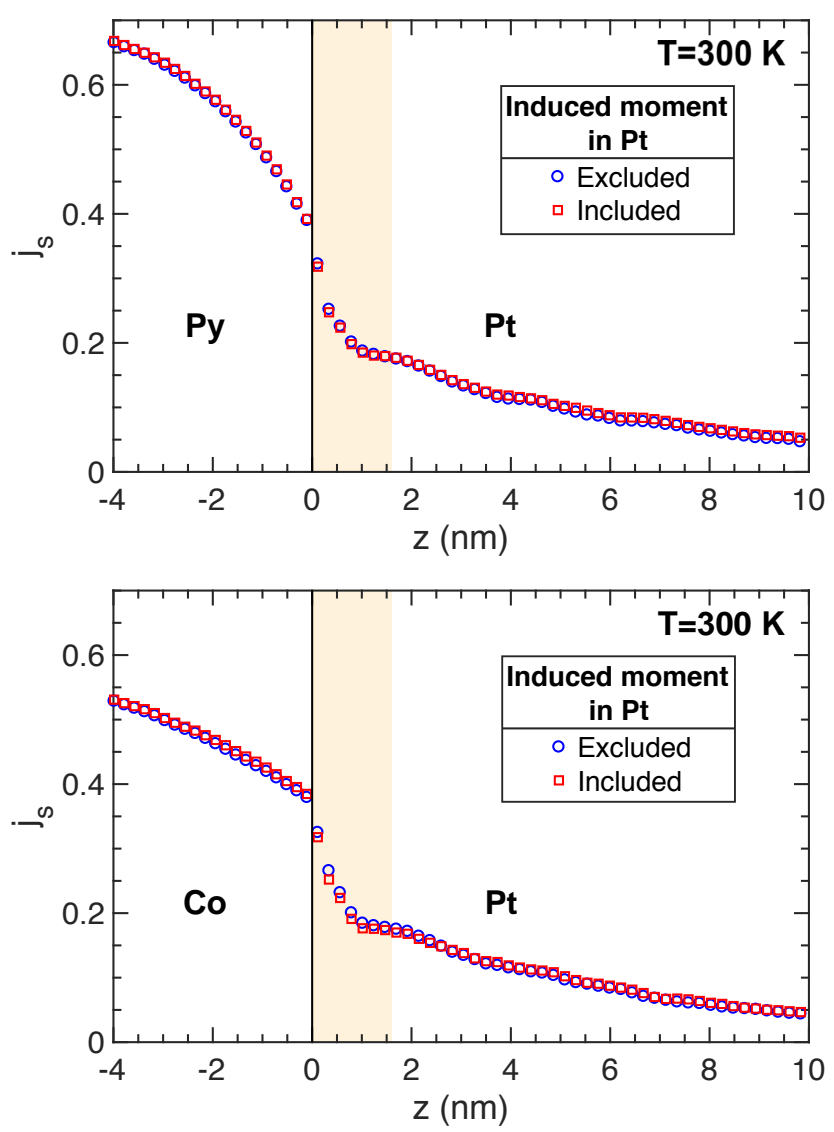

Figure 4.12: Spin currents in (upper panel) Py|Pt and (lower panel) Co|Pt with (red squares) and without (blue circles) induced magnetic moments in Pt. The yellow shaded region consists of $7 \mathrm{Pt}$ layers adjacent to the FM that that are magnetized. The magnetic Pt potentials are calculated self-consistently for a $\mathrm{Pt}|\mathrm{Co}| \mathrm{Pt}$ trilayer. The induced moments go from $0.25 \mu_{B}$ to $0.0006 \mu_{B}$ from left to right in the yellow region. 


\subsection{Comparison with other experiments and calculations}

A direct confrontation of our results for the temperature dependence of the interface parameters with experiment is not possible for a number of reasons.

(1) CPP-MR experiments are conventionally described in terms of the eight VF parameters we have extracted [17]. However, in these experiments the multilayers are usually created by sputtering that leads to intermixing of the materials forming the interface rather than atomically sharp interfaces. While it might be tempting to extend the present study to intermixed interfaces, the distribution of atomic species about the interface is not known; we have to restrict ourselves to ordered interfaces. CPP-MR measurements are restricted to low temperatures $(4.2 \mathrm{~K})$ by making use of superconducting $\mathrm{Nb}$ leads. Lateral microstructuring increases the small resistance of a thin layered structure with respect to long leads [7], allowing the interface parameters of epitaxial $\mathrm{Co} \mid \mathrm{Cu}$ [144] and sputtered $\mathrm{Co}_{50} \mathrm{Fe}_{50} \mid \mathrm{Cu}$ [145] to be determined as a function of temperature up to $300 \mathrm{~K}$. To the best of our knowledge, no similar studies have been carried out for FM|Pt interfaces at finite temperatures.

(2) In SP-ISHE experiments, spins experience enhanced damping at the interface from a combination of SML and spin-backflow [29, 146]. Here it is worth mentioning that the pumped spin current has components polarized both parallel and perpendicular to the FM magnetization. In most studies, only the DC component of the pumped spin is accessed in terms of the DC inverse spin Hall voltage, that is essentially aligned parallel to the magnetization [23, 90]. It has been theoretically predicted that interface spin-orbit coupling can affect spins aligned parallel and perpendicular to the magnetization in different ways [33]. Because we only consider spin memory loss for spins aligned parallel to the magnetization, our estimates of $\delta$ can in principle be used in analyses of SP-ISHE experiments where DC voltages are measured. However, Liu et al. [30] estimated $\delta=3.7$ for Py|Pt interface at $300 \mathrm{~K}$ to account for the interface damping enhancement determined from first-principles "energy pumping" calculations [46]. The spin currents studied in this chapter are generated by spin-polarization of charge currents due to the ferromagnet layer, whereas the spin currents in SP-ISHE experiments and in the work by Liu et al., spin currents are generated by spin-pumping. More work is required to understand whether $\delta$ for "pumped" spin currents is different from $\delta$ for "spin-polarized" currents. Additionally, a large interface contribution to the inverse spin-Hall effect is expected [32]. At present, experiment is unable to disentangle these effects.

(3) In SHE-STT experiments, the in-plane charge current passing through the NM layer gives rise to a spin current that is polarized perpendicular to the charge current and the interface normal direction. This current would be modified at the FM|NM interface by spin memory loss and eventually an interface spin Hall effect. Spin currents are also expected to be generated by spin-orbit filtering and 
precession [113] at the interface that could exert additional torques on the FM. Such a scenario is described by a phenomenological model [33, 34] expressed in terms of a set of parameters larger than current experiments are able to evaluate.

It is nevertheless worth briefly discussing experiments that determine bulk parameters such as the spin-flip diffusion length and the spin Hall angle where interface effects are considered to critically influence the determination of the bulk parameters. These interface effects are expressed in terms of the parameters we have calculated.

\section{Interface resistance and interface spin-asymmetry}

$R_{\mathrm{I}}$ and $\gamma$ are relevant for CPP-MR experiments. These parameters have been extracted for the Co|Pt interface at $4.2 \mathrm{~K}$ by Sharma et al. [147] and by Nguyen et al. [15]. The former did not include $\delta$ in their VF analysis. The latter reported $A R_{\mathrm{I}}=$ $0.53 \pm 0.21 \mathrm{f} \Omega \mathrm{m}^{2}\left[A R_{\mathrm{I}}^{*}\right.$ converted to $A R_{\mathrm{I}}$ using the relation: $\left.A R_{\mathrm{I}}=A R_{\mathrm{I}}^{*}\left(1-\gamma^{2}\right)\right]$ and $\gamma=0.53 \pm 0.12$. We are not aware of any studies of $R_{\mathrm{I}}$ and $\gamma$ in Py|Pt.

\section{Spin memory loss}

Very few experiments have been carried out at finite temperatures that either take SML into account or recognize its role in determining other bulk parameters. Nguyen et al. [15] carried out CPP-MR measurements for sputtered Co|Pt at $4.2 \mathrm{~K}$ and reported $\delta_{\mathrm{Co|Pt}}=0.9_{-0.2}^{0.5}$. Earlier, values of $\delta$ in the range $0.2-0.35$ were found for ColNM pairs with NM metals whose SOC is weaker than that of Pt [10].

Rojas-Sanchez et al. [29] incorporated Nguyen's low temperature interface parameters $\delta$ and $R_{\mathrm{I}}$ for Co|Pt into the analysis of their SP-ISHE experiments to demonstrate that neglecting interface effects leads to underestimation of bulk parameters. Zhang et al. [35] introduced an interface transparency parameter to measure the efficiency of spin-Hall induced spin current transfer from the NM metal to the FM metal. From ST-FMR measurements, they predicted interface transparencies of $0.25 \pm 0.05$ and $0.65 \pm 0.06$ y for Py|Pt and Co|Pt, respectively, the opposite of the trend we found for the interface resistance. Recently, Tao et al. [37] reported $\delta$ for Py|Pt and Co|Pt as $0.63 \pm 0.05$ and $0.39 \pm 0.01$ respectively from SP-ISHE experiment. Berger et al. [38] attributed a 60\% loss of damping enhancement measured using "Vector Network Analyzer-FMR" technique at a Py|Pt interface to SML.

\subsection{Conclusions}

We have developed a practical scheme for calculating the temperature dependence of bulk and interface transport parameters for real materials incorporating all of the complexity of the electronic structure of transition metals both nonmagnetic and magnetic. We illustrated it with a study of Py|Pt and Co|Pt interfaces for which 
we determined all eight VF parameters. Bulk spin flip diffusion lengths could be identified as such for Py, Co and Pt and were determined independent of interface contributions. Thermal and magnetic disorder facilitates the transmission of electrons through the interface, yet doesn't render $A R_{\mathrm{I}}$ and $\delta$ zero even at high temperature. Finally, induced magnetic moments in Pt do not affect the interface discontinuity. 


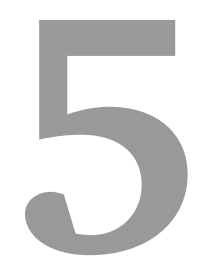

\section{Intrinsic spin-Hall effect in Permalloy*}

The spin Hall effect (SHE) is commonly associated with semiconductors and nonmagnetic metals. In the last few years, several experiments have reported the observation of an inverse SHE in $3 d$ ferromagnetic alloys and metals, in particular for permalloy (Py). The underlying spin currents that generate the ISHE are oriented along the magnetization direction of Py. Some studies also report a non-trivial SHE in permalloy such that the spin-polarization is not oriented along the magnetization; its origin in a $3 d$ magnetic alloy is not known. In this chapter, we investigate SHE in permalloy that is independent of the magnetization direction using first-principles scattering calculations and local spin currents derived from the resulting wavefunctions. By examining the spin Hall angle as a function of energy about the Fermi energy for a virtual crystal approximation of permalloy, we can trace the origin of the SHE in permalloy to a very flat band in the X-W-L-K region of the Brillouin zone and the spin-orbit splittings of orbitally degenerate minority-spin bands at the $X$ and $L$ points.

\footnotetext{
"In preparation: K. Gupta, R.S.Nair, Z. Yuan, K. Chen, P.J. Kelly, Intrinsic spin-Hall effect in Permalloy
} 

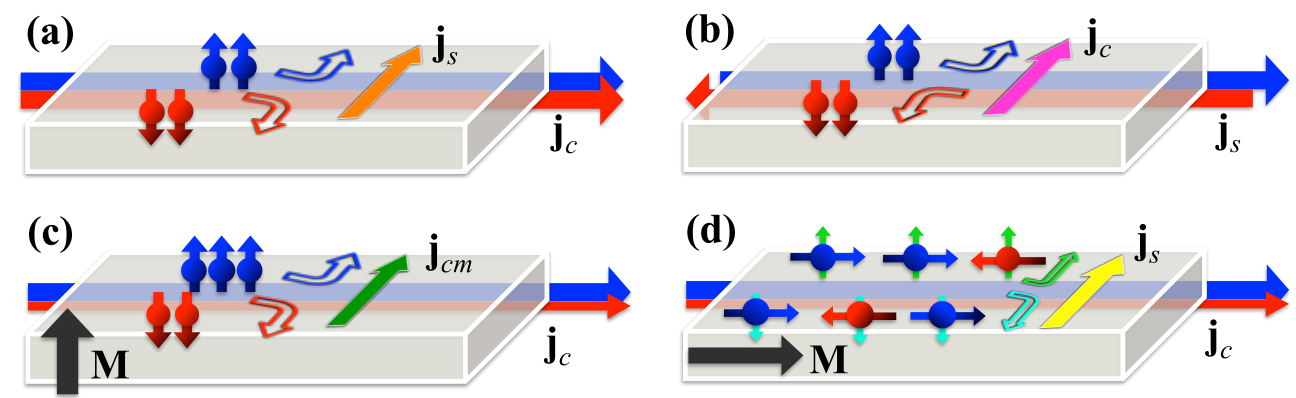

Figure 5.1: Schematic diagrams of (a) the spin Hall effect and (b) inverse spin Hall effect in a nonmagnetic material, (c) the anomalous Hall effect in a ferromagnet for magnetization $\mathbf{M}$ perpendicular to the charge current $\mathbf{j}_{c}$ and (d) the proposed spin Hall effect in ferromagnet for $\mathbf{M} \| \mathbf{j}_{c}$.

\subsection{Introduction}

The spin Hall effect (SHE), inverse spin Hall effect (ISHE) and anomalous Hall effect (AHE) share a common relativistic origin - the spin orbit interaction (SOI). In nonmagnetic materials, the SHE gives rise to a spin current $\mathbf{j}_{s \alpha}$ transverse to a charge current $\mathbf{j}_{c}$, and leads to spin-up and spin-down electrons accumulating at opposite edges of a sample as shown in Fig. 5.1(a). The polarization of the spins, denoted $\alpha$, is mutually perpendicular to $\mathbf{j}_{c}$ and $\mathbf{j}_{s}$. Conversely, a spin current $\mathbf{j}_{s \alpha}$ with a polarization perpendicular to the direction of the current $\hat{\mathbf{j}}_{s}$ generates a mutually orthogonal charge current $\mathbf{j}_{c}$ via the ISHE, Fig. 5.1(b). In a ferromagnet, with an unequal number of spin-up and spin-down electrons, an applied charge current $\mathbf{j}_{c}$ generates a transverse current $\mathbf{j}_{c m}$ perpendicular to $\mathbf{j}_{c}$ and the magnetization direction $\mathbf{M}$ of the ferromagnet in what is called the AHE. $m$ in $\mathbf{j}_{c m}$ indicates that the charge current is spin-polarized along $\mathbf{M}$ because of spin asymmetry, as shown in Fig. 5.1(c).

In the last few years, an ISHE has been observed in ferromagnetic alloys such as permalloy $\left(\mathrm{Py}=\mathrm{Ni}_{80} \mathrm{Fe}_{20}\right)$ [39, 40, 148-150] and $\mathrm{Co}_{90} \mathrm{Fe}_{10}$ [151]. A few studies have estimated the spin Hall angle (SHA) from ISHE measurements for various $3 d$ metals as well as for Py [42, 152]. In all of these ISHE experiments, the incident spin current is polarized along the magnetization direction. In a ferromagnet where the spin polarization and magnetization are inextricably coupled, the ISHE can be said to be the reciprocal of the AHE, compare Figs. 5.1(b) and 5.1(c). In nonmagnetic materials, the SHE is the reciprocal of the ISHE because there is no preferential spin orientation. Subsequent studies have focussed on the spin polarized currents generated by the AHE in NiFe $[41,150]$ and $\mathrm{CoFeB}[153,154]$ as an alternative to SHE-induced spin currents in nonmagnets because the former can be manipulated by changing the magnetization orientation. 
It has also been shown experimentally that SHE can be present in a ferromagnet such that a transverse spin current is generated in response to a charge current with spins polarized perpendicular to the magnetization $\mathbf{M}[41,42]$. This case is sketched in Fig. 5.1(d). Another study shows that the ISHE in Co remains unchanged if $\mathbf{M}$ is reversed [155]. These few studies suggest that spin Hall effects in ferromagnets may not be solely coupled to their magnetization as assumed for most ISHE studies [39, 40, 148-150]. It is commonly expected that any component of a spin current polarized perpendicular to the magnetization must dephase [156]. Very recently, a theoretical study has reported that in $3 \mathrm{~d}$ ferromagnets $\mathrm{Co}, \mathrm{Ni}$ and $\mathrm{Fe}$, SHE is given by a sum of "magnetization orientation independent" as well as magnetization orientation dependent contributions[157].

Because Py is a widely used ferromagnet, our focus in this Chapter will be to demonstrate the existence of a transverse spin current resulting from SHE in Py that is independent of the magnetization direction using first-principles calculations at $T=0 \mathrm{~K}$. With respect to both current direction and polarization, this spin current is orthogonal to the spin current associated with the primary driving charge current that is polarized parallel to the magnetization $\mathbf{M}$ (Sec. 5.3.1). The magnitude of the spin Hall angle (SHA) is comparable to that reported in Sec. 2.3.4 for Pt. By systematically varying the lateral supercell size, we also find no evidence for dephasing of this spin current (Sec. 5.3.2). Calculations at different temperatures up to $600 \mathrm{~K}$ show that the SHE in Py is robust with respect to thermal lattice and spin disorder (Sec. 5.3.3). Finally, we address the origin of the SHE in Py in terms of its electronic structure (Sec. 5.4).

\subsection{Calculations}

The quantum mechanical scattering problem [120] is solved for a scattering region consisting of $\mathrm{Ni}$ and $\mathrm{Fe}$ atoms in the ratio 80:20 embedded between ballistic $\mathrm{Cu}$ leads. The full quantum mechanical wavefunction is obtained using a wavefunction matching scheme [47] implemented [45, 48] with a tight-binding (TB) muffin-tin orbital (MTO) basis [51, 74, 75] and generalized to include spin-orbit coupling and noncollinearity $[46,72]$ as well as temperature induced lattice and spin disorder $[66,67]$. The wavefunction obtained for the scattering region is used to determine position dependent charge and spin currents as described in Sec. 2.2. Calculations are carried out with an $s p d$ orbital basis and including both two and three center terms in the SOC Pauli Hamiltonian $\xi$ l.s [46]. A number of computationally expensive test calculations were carried out with only two center terms to reduce the computational costs by $70 \%$.

The $\mathrm{Cu}$ lead potential was calculated in the atomic spheres (AS) approximation (ASA) using the TB-LMTO method [51, 74, 75]. AS potentials for Ni and Fe were evaluated self-consistently for a substitutional random alloy of fcc Py using the co- 
herent potential approximation (CPA) [54] implemented with TB-MTOs [53]. The resulting $\mathrm{Ni}$ and $\mathrm{Fe}$ AS potentials were used to randomly populate fcc lattice sites in lateral supercells transverse to the [001] transport direction. The percentages of the total numbers of $\mathrm{Ni}$ and $\mathrm{Fe}$ atoms in the scattering region is $80 \%$ and $20 \%$, respectively, and no symmetry is assumed in the transport direction. As discussed in Sec. 2.3.2, a moderately sized $(5 \times 5)$ lateral supercell is sufficient to describe various transport parameters for Py. The calculations in Secs. 5.3.1, 5.3.3 and 5.4 were carried out using $6 \times 6$ supercells. In Sec. 5.3.2, we test the effect of varying the supercell size on the transverse spin currents. For all calculations, the 2D Brillouin zone was sampled with $Q \times Q k$-points so that for an $N \times N$ supercell, the effective sampling for a $1 \times 1$ primitive unit cell is $N Q \times N Q$ with $N Q \sim 190$.

To carry out finite temperature calculations in Sec. 5.3.3, we use a frozen thermal disorder scheme $[46,66,67]$ to displace atoms from their equilibrium positions and rotate magnetic moments from their equilibrium orientations. The distribution of the (uncorrelated) atomic displacements is assumed to be Gaussian and is characterized by a root-mean square displacement $\Delta . \Delta(T)$ at a given temperature $T$ is derived from the Debye model [138] for Py whose Debye temperature $=450 \mathrm{~K}$. Spin disorder is modelled with a Gaussian distribution of rotations to reproduce the experimental magnetization [139] for a given temperature. For a given value of $\Delta$, ten random configurations of disorder are generated and all results are averaged over these configurations.

\subsection{Results}

\subsubsection{Spin-Hall effect in Py at $0 \mathrm{~K}$}

We pass a charge current $\mathbf{j}_{c}$ along the transport direction $z$ through a (001) oriented $120 \mathrm{~nm}$ long slab of Py embedded between ballistic $\mathrm{Cu}$ leads at $T=0 \mathrm{~K}$. The Py magnetization is oriented parallel to the transport direction. The full spin current tensor $\mathbf{j}_{s \alpha}$ is obtained from the scattering calculations described in Sec. 5.2 for 10 configurations of disorder. The index $\alpha \equiv x, y, z$ represents the orientation of the spin polarization and $\mathbf{j} \equiv\left(j^{x}, j^{y}, j^{z}\right)$ represents the current direction. Of all the components of $\mathbf{j}_{s \alpha}$, the ones found to be significantly large are plotted in Fig. 5.2 and discussed as follows.

The charge current $\mathbf{j}_{c}$ carries the polarization of the equilibrium magnetization; the resulting spin current, polarized along $z$ and normalized to the constant charge current $j_{c}$ is denoted $j_{s z}^{z}(z)$ and is plotted as black circles in Fig. 5.2. The center of the data symbols corresponds to the mean of the spin currents obtained by averaging over ten configurations of chemical disorder and their size corresponds to the spread expressed as the mean absolute deviation. The $z$ dependence of $j_{s z}^{z}(z)$ is described by the Valet-Fert parameters $\beta$, the spin-polarization or spin-asymmetry, and $l_{\mathrm{sf}}$, the spin-flip diffusion length. By choosing the center of the FM slab to be 


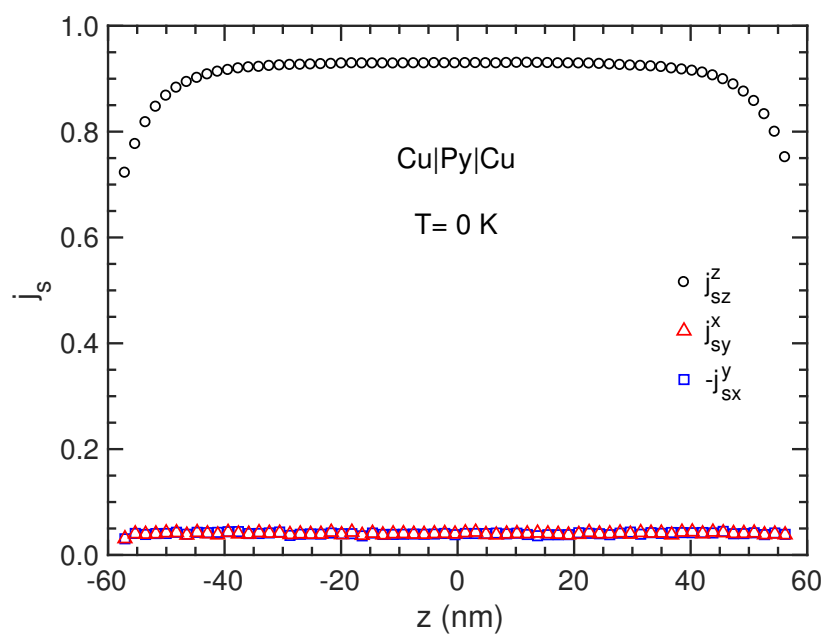

Figure 5.2: A charge current $j_{c}$ passed through (001) oriented fcc Py polarizes because of spin-asymmetry. The Py magnetization is directed along $z$. The spatial variation of this spin-polarized current $j_{s z}^{z}(z)$ normalized to the constant $j_{c}$ is plotted as black circles. The charge current induces transverse spin currents $j_{s y}^{x}$ and $j_{s x}^{y}$ shown as red triangles and blue squares respectively. All currents are averaged over 10 configurations of chemical disorder indicated by center of data symbols. The symbol size indicates the absolute mean deviation. For clarity, the data symbols are only shown for every tenth atomic layer.

the origin $z=0$ and fitting $j_{s}(z) \equiv j_{s z}^{z}(z)$ to the solution of the Valet-Fert equations $j_{s}(z)=\beta-c \cosh \left(z / l_{\mathrm{sf}}\right)$, we find $\beta=0.9289 \pm 0.0001$ and $l_{\mathrm{sf}}=6.34 \pm 0.05 \mathrm{~nm}$ for Py at $0 \mathrm{~K}$.

Transverse spin currents are found in the $x$ and $-y$ directions that are polarized in the $y$ and $x$ directions, respectively. These two transverse currents, normalized to the longitudinal charge current $j_{c}$ and denoted $j_{s y}^{x}(z)$ and $-j_{s x}^{y}(z)$, are plotted in Fig. 5.2 as red triangles and blue squares. Because a magnetization along $z$ will otherwise not lead to polarization along $x$ or $y$, these spin currents must result from the spin Hall effect which gives rise to spin currents in the transverse direction. The polarization direction of the spin current is given by a vector product of the original current direction $(z)$ and the transverse spin current direction. Within the small error bars, the magnitudes of $j_{s y}^{x}(z)$ and $-j_{s x}^{y}(z)$ are identical even though axial symmetry is only restored after averaging.

Because $j_{s y}^{x}(z)$ and $-j_{s x}^{y}(z)$ exhibit barely any spatial $(z)$ dependence, unlike $j_{s z}^{z}(z)$, we can use shorter scattering region lengths to study the SHE in the remainder of this chapter. The transverse spin currents calculated for an $18 \mathrm{~nm}$ thick slab are shown in Fig. 5.3. We extract the bulk value of the spin-Hall an- 


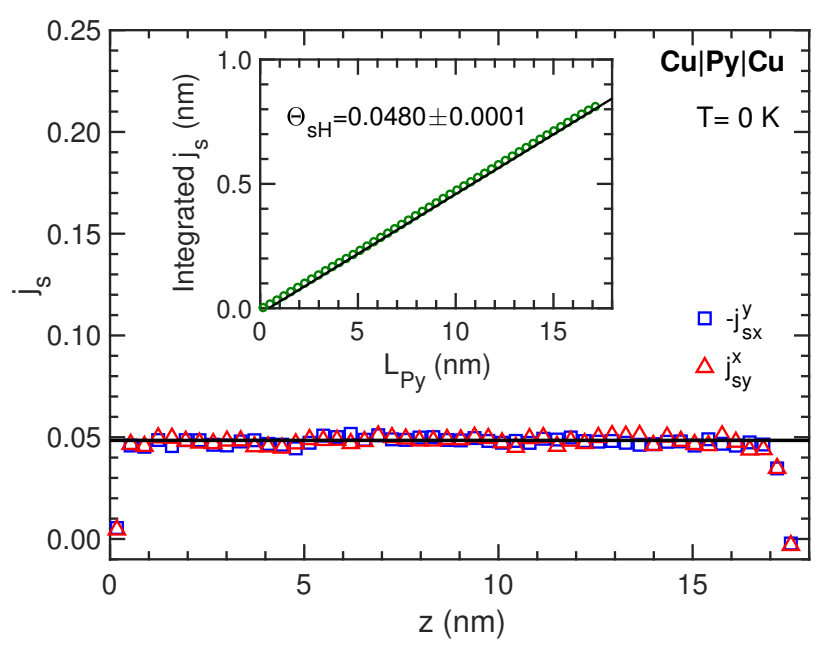

Figure 5.3: Transverse spin currents driven by a charge current in the $z$ direction for a (001) oriented $T=0 \mathrm{~K}$ Py slab embedded between $\mathrm{Cu}$ leads. The Py magnetization is directed along $z .6 \times 6$ supercells were used with a $32 \times 32 \mathrm{BZ}$ sampling. The currents are averaged over ten configurations of disorder and data symbols are only shown for every second atomic layer for clarity. The symbol size indicates the mean absolute deviation. The horizontal solid line indicates the extracted value of $\Theta_{\mathrm{sH}}$. Inset: integrated transverse spin currents $J_{s}^{\perp}$ as a function of $L_{\mathrm{Py}}$. The solid line indicates the weighted linear least squares fit that results in $\Theta_{\mathrm{sH}}=0.0480 \pm 0.0001$. 


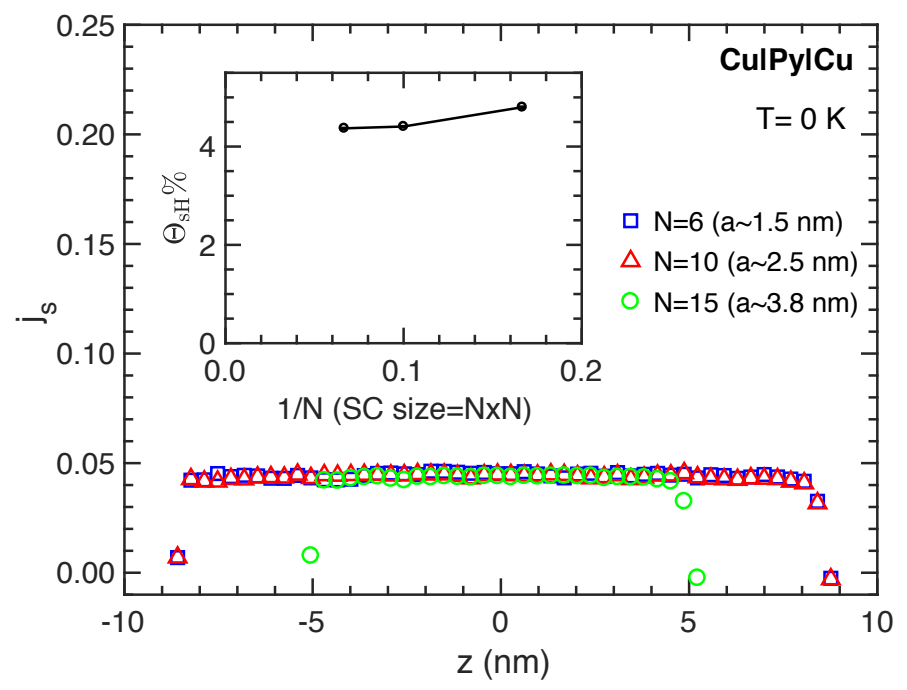

Figure 5.4: Direction averaged transverse spin current $j_{s}=\left(j_{s y}^{x}-j_{s x}^{y}\right) / 2$ in Py for different $N \times N$ supercell sizes $(N=6,10,15)$. Three-center terms in the SOC Hamiltonian are omitted in these calculations. The slab lengths are 18, 18 and $10 \mathrm{~nm}$ for $\mathrm{N}=6,10$ and 15 respectively. $t$ is the dimension of the square lateral supercell for fcc (001) Py. Inset: $\Theta_{\mathrm{sH}} \%$ extracted from the fits of integrated $j_{s}$ are shown as a function of $1 / N$.

gle, $\Theta_{\mathrm{sH}}$, as follows. Starting from the left interface at $z=0$, the configuration averaged $j_{s y}^{x}(z)-j_{s x}^{y}(z)$ is integrated over atomic layers up to some $L_{\mathrm{Py}}: J_{s}^{\perp}\left(L_{\mathrm{Py}}\right)=$ $\int_{0}^{L_{\mathrm{Py}}}\left[j_{s y}^{x}(z)-j_{s x}^{y}(z)\right] / 2 d z$. The integral is shown as a function of $L_{\mathrm{Py}}$ in the inset to Fig. 5.3 as green circles. A linear least squares fit yields a value of $\Theta_{\mathrm{sH}}=$ $4.80 \pm 0.01 \%$ as the slope. The error bar results from the weighted residuals where the weights are the mean absolute deviation for 10 configurations of chemical disorder. Compared to the spin polarization $\beta=92.89 \%$ induced by the magnetization, the transverse spin polarization of $4.8 \%$ is much smaller. However, this spin-Hall angle is very similar to the value $\Theta_{\mathrm{sH}}^{\mathrm{Pt}}=5.0 \pm 0.1 \%$ obtained in Sec. 2.3.4 for Pt with an spd basis and three center SOC terms.

\subsubsection{Effect of SC size}

Even though it is significantly smaller than $\beta$, a finite value of $\Theta_{\mathrm{sH}}$ for a ferromagnetic material is non-trivial because it is generally assumed that any spins with polarization perpendicular to the magnetization direction must rapidly dephase inside the bulk of a ferromagnet. Use of a finite lateral supercell in our calculations poses the question as to whether the supercell size is large enough to observe any 


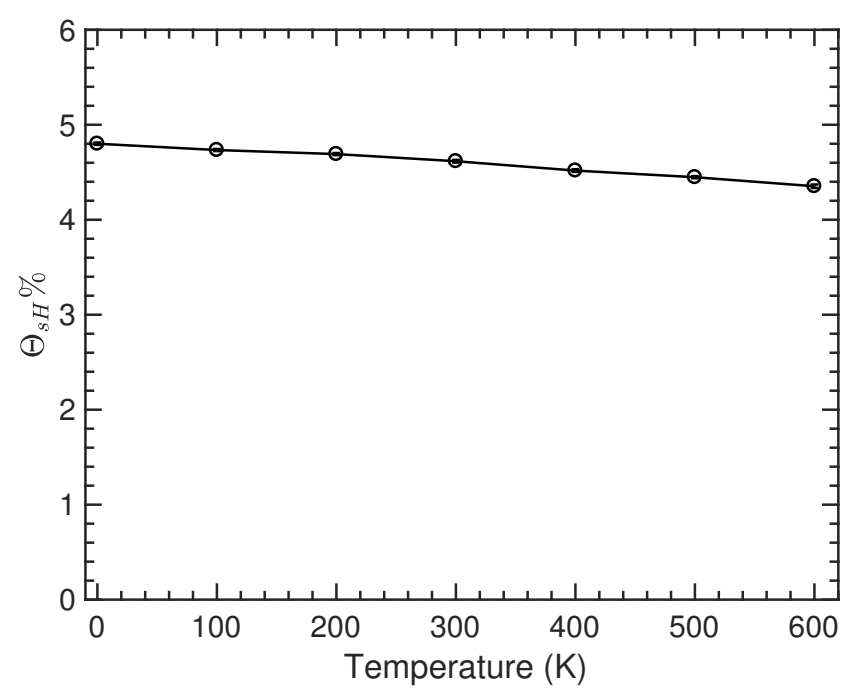

Figure 5.5: Spin-Hall angle for (001) oriented Py as a function of temperature.

eventual dephasing. To test this, we calculate $\Theta_{\mathrm{SH}}$ as a function of the supercell size. Because this is computationally very expensive, we neglected the three-center SOC terms. The results are shown in Fig. 5.4. The $6 \times 6$ supercell used to obtain the results shown in Fig. 5.3 has dimensions of a square with side $a=a_{\mathrm{Py}} / \sqrt{2} \times 6=1.5$ $\mathrm{nm}\left(a_{\mathrm{Py}}=3.54 \mathrm{~nm}\right)$. We carried out calculations for $10 \times 10$ and $15 \times 15$ supercells corresponding to $t \sim 2.5 \mathrm{~nm}$ and $t \sim 3.8 \mathrm{~nm}$, respectively. The values for $\Theta_{\mathrm{sH}}$ are shown in the inset to Fig. 5.4 and display very little dependence on the supercell size. Thus, on a length scale of order $1 \mathrm{~nm}$ there are no indications of dephasing of this spin current. Amin et al. [157] suggest that intrinsic spin currents are not subject to dephasing because they result from perturbed eignstates whose spin components do not acquire a relative phase as they propagate, that would have otherwise lead to dephasing.

\subsubsection{Spin-Hall effect in Py as a function of temperature}

We now study how $\Theta_{\mathrm{sH}}$ behaves as a function of temperature for Py. Two- and three-center SOC terms are once again included in these calculations. As shown in Fig. 5.5, a weak temperature dependence is found. $\Theta_{\mathrm{sH}}$ decreases from $4.8 \pm 0.1 \%$ at $0 \mathrm{~K}$ to $4.35 \pm 0.02 \%$ at $600 \mathrm{~K}$. It would be interesting to see if this decrease becomes more rapid close to the Curie temperature of $\mathrm{Py}=872 \mathrm{~K}$ or is enhanced as has recently been suggested [158]. 


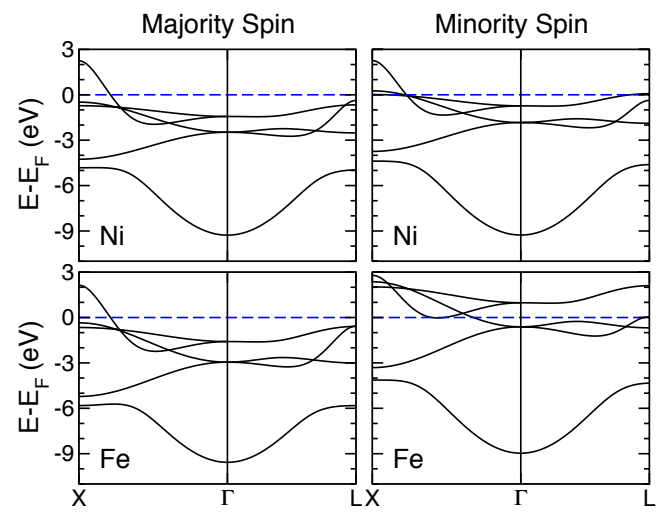

Figure 5.6: Band structures calculated with the Ni and Fe AS potentials and Fermi energy that were calculated self-consistently for $\mathrm{Ni}_{0.8} \mathrm{Fe}_{0.2}$ using the coherent potential approximation. The same AS radii were used for $\mathrm{Ni}$ and $\mathrm{Fe}$.

\subsection{Microscopic origin of the spin Hall effect in Py}

The above study establishes that there is a spin Hall effect in Py that is comparable in magnitude to that found in Pt, that is not affected by dephasing and that shows a very weak temperature dependence up to two thirds of the Curie temperature. The large size of the SHA in Pt (W and Ta) is usually attributed to the size of the SOC in the $5 d$ elements $[84,105]$. In the virtual crystal approximation (VCA), the nuclear charge of Py is $Z_{\mathrm{VCA}}=(26+4 \times 28) / 5=27.6$ and its spin-orbit coupling parameter $\xi$ is about six times smaller than that of Pt with $Z=78$ [159]. Two experimental $[42,152]$ studies and a theoretical [160] study systematically investigated SHA for various $3 \mathrm{~d}$ transition metals. A common observation is that $\mathrm{SH}$ conductivities change sign from negative to positive as the $d$-orbital filling increases, a trend similar to that found for $4 d$ and $5 d$ transition metals [84, 105]. The reported SH conductivities are not much smaller than those found for $4 d$ or $5 d$ transition metals. So far, no direct explanation has been given for why the SHE is so large in a $3 d$ alloy. In the remainder of this section, we will address this question.

The largest contributions to the intrinsic anomalous Hall effect in ferromagnetic metals [161] and spin Hall effect in nonmagnetic metals [12] come from the spin-orbit splitting of bands near the Fermi energy. We will argue that the large $\Theta_{\mathrm{sH}}$ in Py also comes from such a splitting. In a substitutional disordered alloy like Py, translational symmetry is broken even at $T=0 \mathrm{~K}$ so that Bloch's theorem does not hold and a band structure as such does not exist. In the coherent potential approximation (CPA) [54], translational symmetry is restored by introducing a single effective, "coherent potential" on every site in the lattice so eigenstates can again be characterized with a Bloch wavevector $\mathbf{k}$. The coherent potential is complex and energy dependent so that the energy eigenvalues are no longer real and the 

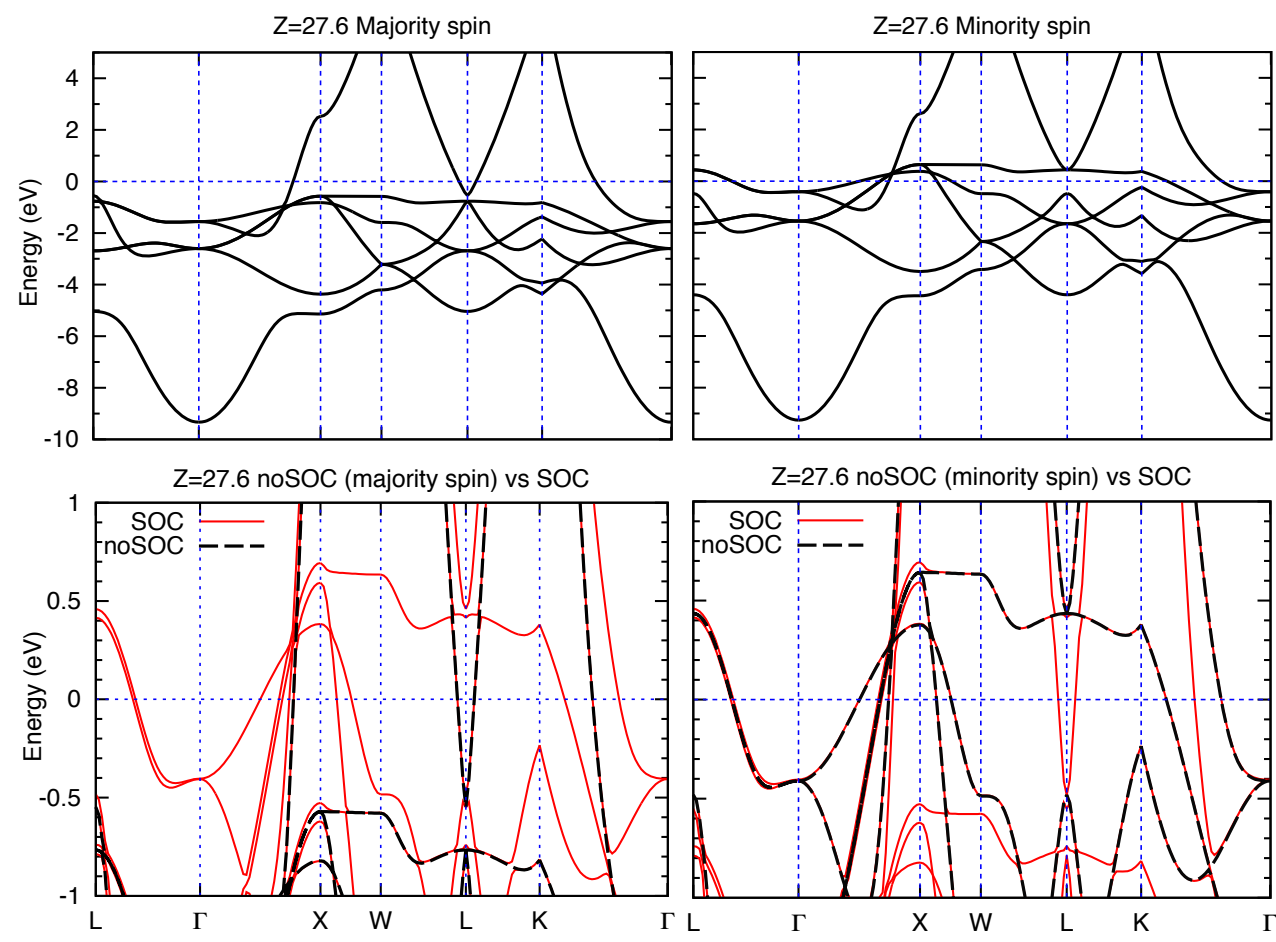

Z=27.6 noSOC (minority spin) vs SOC

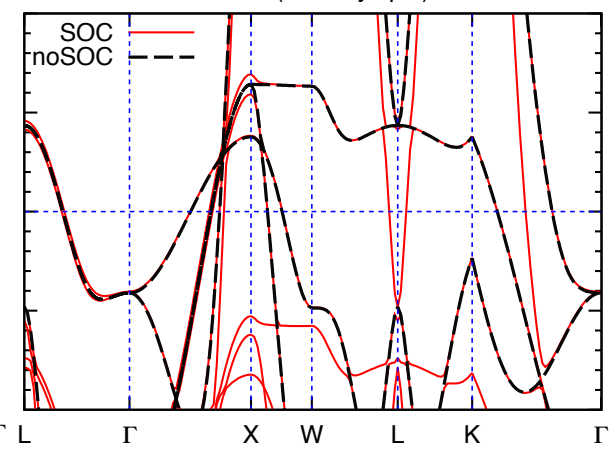

Figure 5.7: Upper panels: (lhs) Majority and (rhs) minority bands for VCA Py evaluated without SOC. Lower panels: (lhs) Majority and (rhs) minority bands evaluated without SOC (black dashed lines) compared with spin-mixed bands evaluated with SOC (red solid lines) for VCA Py.

eigenstates are described as having a finite disorder-induced lifetime.

To understand the electronic structure of Py, we plot in Fig. 5.6 the energy bands corresponding to the optimized Fe and Ni AS potentials that are calculated self-consistently with the TB-LMTO-CPA method [53]. At the Fermi energy, the majority-spin bands for $\mathrm{Ni}$ and Fe are almost identical so that in a disordered alloy the majority-spin electrons see essentially the same potentials on all lattice sites and are only very weakly scattered by the randomly distributed $\mathrm{Ni}$ and Fe potentials. In contrast, the minority-spin bands are quite different for $\mathrm{Ni}$ and $\mathrm{Fe}$, which can be understood in terms of the different exchange splittings; the magnetic moments calculated for $\mathrm{Ni}$ and Fe in permalloy in the CPA are 0.63 and $2.61 \mu_{\mathrm{B}}$, respectively. The random distribution of $\mathrm{Ni}$ and Fe potentials in permalloy then leads to strong scattering of minority-spin electrons in transport. Of particular interest in the present context are the very flat minority spin $d$ bands that straddle the Fermi energy for $\mathrm{Fe}$ and $\mathrm{Ni}$ and the twofold orbital degeneracies at the $\mathrm{X}, \Gamma$ and $\mathrm{L}$ points.

To relate the SHE in Py to an electronic structure, we need to simplify our model 
and to do so, we use the VCA introduced above. With a "Py" nuclear charge of 27.6 and a corresponding number of valence electrons, self consistent calculations are carried out with a TB-LMTO-ASA code ${ }^{\dagger}$ for an fcc lattice with the same lattice constant as Py. The magnetic moment of VCA-Py is $1.04 \mu_{B}$ compared to $1.01 \mu_{B}$ for CPA-Py. We first need to verify that this electronic structure when combined with disorder can generate a SHE. Using thermal lattice and spin disorder appropriate to RT Py, we calculate the SHA for VCA-Py and find a value of $\Theta_{\mathrm{Py}} \equiv \Theta_{\mathrm{sH}}^{\mathrm{Py}} \sim 2 \%$. While this is only half the value of what we calculate for CPA-Py, it is still substantial and adequate for the present purpose of identifying the features in the electronic structure that give rise to such a large effect in a $3 d$ system.

The VCA bands obtained without SOC are shown in the upper panel of Fig. 5.7. The feature that immediately attracts our attention in both the majority spin (lhs) and minority spin (rhs) cases is the uppermost $d$ band that is remarkably dispersionless in a large volume of the Brillouin zone (BZ), from X-W-L-K in the figure. In particular we note the twofold orbital degeneracy at the $\mathrm{X}$ and $\mathrm{L}$ points. By analogy with Pt [84], we might expect these doubly degenerate states to be strongly affected by SOC. In the lower panel of Fig. 5.7, we plot the bands obtained on including SOC self-consistently for VCA-Py. On the left- and right-hand sides, we superimpose the corresponding majority and minority spin bands obtained without SOC. We immediately see that the double degeneracies at $\mathrm{X}$ and $\mathrm{L}$ are lifted by SOC with splittings of $\sim 0.10 \mathrm{eV}$ and $\sim 0.05 \mathrm{eV}$ respectively. If the large SHA can be associated with the lifting of degeneracies, this would explain the larger values of $\Theta_{\mathrm{sH}}$ reported for Ni compared to Fe and Co in experiment [42] and [157].

To study how $\Theta_{\mathrm{sH}}$ depends on these splittings, we calculate $\Theta_{\mathrm{sH}}$ using the same procedure described in Sec. 5.2 for thermally disordered VCA-Py as a function of the energy in a rigid band approximation. Because Py is a strong ferromagnet, nothing changes in the majority spin bands when the composition is varied about that of Py. We choose the same lattice and spin disorder as for Py at $300 \mathrm{~K}$ and for computational efficiency, we only include SOC two center terms. We obtain $\Theta_{\mathrm{sH}}=2 \%$ for VCA-Py compared to $\Theta_{\mathrm{sH}}=4.3 \%$ for CPA-Py (only including two center terms) at $300 \mathrm{~K}$. Assuming a rigid band, we artificially move $E_{F}$ through the band to see how $\Theta_{\mathrm{sH}}$ varies as the $E_{F}$ moves closer to splittings in $\mathrm{X}$ and $\mathrm{L}$. The results are shown in Fig. 5.8. Even though the energy sampling is very coarse, $\Theta_{\mathrm{sH}}$ indeed follows the density of states (DoS) and spin-orbit splitting of minority spins as $E_{F}$ is moved up in energy. We see that as long as the minority spins have a respectable DoS, $\Theta_{\mathrm{sH}}$ is significant.

\footnotetext{
"We use Mark van Schilfgaarde's "lm" extension of the Stuttgart LMTO code that treats noncollinear magnetization and spin-orbit coupling and is maintained in the "QUESTAAL" suite at https://www.questaal.org.
} 


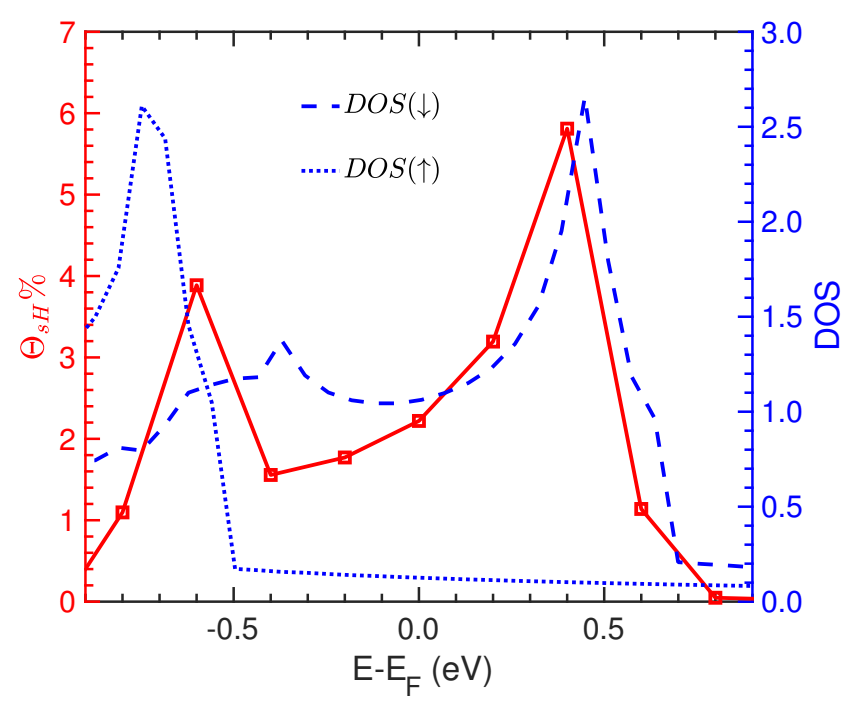

Figure 5.8: Spin Hall angle $\Theta_{\mathrm{sH}}$ for Py as a function of the energy in a rigid band approximation (in red squares). The blue dashed line indicates the density of states (DoS) for minority spins. The blue dotted line indicates the DoS for majority spins.

\subsection{Summary}

In this Chapter, we have demonstrated a substantial spin Hall angle in permalloy that is independent of the magnetization direction. For temperatures between 0 and $600 \mathrm{~K}$, the SHA ranges from $4.8 \%$ to $4.35 \%$. This value is comparable to the spin Hall angle of Pt at $300 \mathrm{~K}, \Theta_{\mathrm{sH}} \sim 5.0 \%$, that is commonly attributed to the large size of its SOC. Our calculations support the recent findings [42, 157] that a large SHA can exist for $3 d$ metals as well. By investigating the band structure of Py in the virtual crystal approximation, we found that there exist spin-orbit splittings at the $X$ and $L$ high symmetry points for minority spin bands. The SHA of Py in the virtual crystal approximation was calculated using $\mathrm{T}=300 \mathrm{~K}$ thermal lattice and spin disorder as a function of energy in a rigid band approximation around the Fermi energy. When the Fermi energy is close to the $X$ and $L$ points, the SHA was maximum thus establishing the link between spin-orbit splittings and SHA. 


\section{Bibliography}

[1] N. F. Mott, "The electrical conductivity of transition metals", Proc. R. Soc. London, Ser. A 153, 699-717 (1936).

[2] M. N. Baibich, J. M. Broto, A. Fert, F. Nguyen Van Dau, F. Petroff, P. Etienne, G. Creuzet, A. Friederich, and J. Chazelas, "Giant Magnetoresistance of (001)Fe/(001)Cr Magnetic Superlattices", Phys. Rev. Lett. 61, 2472-2475 (1988).

[3] G. Binasch, P. Grünberg, F. Saurenbach, and W. Zinn, "Enhanced magnetoresistance in layered magnetic structures with antiferromaganetic interlayer exchange", Phys. Rev. B 39, 4828-4830 (1989).

[4] R. E. Camley and J. Barnaś, "Theory of giant magnetoresistance effects in magnetic layered structures with antiferromagnetic coupling", Phys. Rev. Lett. 63, 664-667 (1989).

[5] P. M. Levy, S. F. Zhang, and A. Fert, "Electrical conductivity of magnetic multilayered structures", Phys. Rev. Lett. 65, 1643-1646 (1990).

[6] W. P. Pratt, Jr., S.-F. Lee, J. M. Slaughter, R. Loloee, P. A. Schroeder, and J. Bass, "Perpendicular Giant Magnetoresistance of Ag/Co Multilayers", Phys. Rev. Lett. 66, 3060-3063 (1991).

[7] M. A. M. Gijs, S. K. J. Lenczowski, and J. B. Giesbers, "Perpendicular giant magnetoresistance of microstructured $\mathrm{Fe} / \mathrm{Cr}$ magnetic multilayers from 4.2 to 300 K", Phys. Rev. Lett. 70, 3343-3346 (1993).

[8] P. C. van Son, H. van Kempen, and P. Wyder, "Boundary Resistance of the Ferromagnetic-Nonferromagnetic Metal Interface", Phys. Rev. Lett. 58, 2271-2273 (1987).

[9] T. Valet and A. Fert, "Theory of the perpendicular magnetoresistance in magnetic multilayers", Phys. Rev. B 48, 7099-7113 (1993).

[10] J. Bass and W. P. Pratt Jr., "Spin-diffusion lengths in metals and alloys, and spin-flipping at metal/metal interfaces: an experimentalist's critical review", J. Phys.: Condens. Matter 19, 183201 (2007). 
[11] A. Hoffmann, "Spin Hall effects in metals", IEEE Trans. Mag. 49, 5172-5193 (2013).

[12] J. Sinova, S. O. Valenzuela, J. Wunderlich, C. H. Back, and J. Jungwirth, "Spin Hall effects", Rev. Mod. Phys. 87, 1213-1259 (2015).

[13] R. J. Elliott, "Theory of the effect of spin-orbit coupling on magnetic resonance in some semiconductors", Phys. Rev. 96, 266-279 (1954).

[14] Y. Yafet, "g factors and spin-lattice relaxation of conduction electrons", in Solid State Physics, edited by F. Seitz and D. Turnbull, volume 14, 1-98 (Academic, New York) (1963).

[15] H. Y. T. Nguyen, W. P. Pratt Jr., and J. Bass, "Spin-flipping in Pt and at Co/Pt interfaces", J. Magn. \& Magn. Mater. 361, 30-33 (2014).

[16] D. V. Baxter, S. D. Steenwyk, J. Bass, and W. P. Pratt, Jr., "Resistance and spin-direction memory loss at Nb/Cu interfaces", J. Appl. Phys. 85, 45454547 (1999).

[17] J. Bass, "CPP magnetoresistance of magnetic multilayers: A critical review", J. Magn. \& Magn. Mater. 408, 244-320 (2016).

[18] M. I. Dyakonov and V. I. Perel, "Current-induced spin orientation of electrons in semiconductors", Phys. Lett. A 35, 459-460 (1971).

[19] J. E. Hirsch, "Spin Hall Effect”, Phys. Rev. Lett. 83, 1834 (1999).

[20] S. Zhang, "Spin Hall Effect in the Presence of Spin Diffusion", Phys. Rev. Lett. 85, 393-396 (2000).

[21] T. Kimura, Y. Otani, T. Sato, S. Takahashi, and S. Maekawa, "RoomTemperature Reversible Spin Hall Effect", Phys. Rev. Lett. 98, 156601 (2007).

[22] O. Mosendz, J. E. Pearson, F. Y. Fradin, G. E. W. Bauer, S. D. Bader, and A. Hoffmann, "Quantifying spin Hall angles from spin pumping: Experiments and theory", Phys. Rev. Lett. 104, 046601 (2010).

[23] O. Mosendz, V. Vlaminck, J. E. Pearson, F. Y. Fradin, G. E. W. Bauer, S. D. Bader, and A. Hoffmann, "Detection and quantification of inverse spin Hall effect from spin pumping in permalloy/normal metal bilayers", Phys. Rev. B 82, 214403 (2010).

[24] L. Liu, T. Moriyama, D. C. Ralph, and R. A. Buhrman, "Spin-Torque Ferromagnetic Resonance Induced by the Spin Hall Effect", Phys. Rev. Lett. 106, 036601 (2011). 
[25] D. Wei, M. Obstbaum, M. Ribow, C. H. Back, and G. Woltersdorf, "Spin Hall voltages from a.c. and d.c. spin currents", Nature Communications 5, 3768 (2014).

[26] Y. Tserkovnyak, A. Brataas, and G. E. W. Bauer, "Enhanced Gilbert Damping in Thin Ferromagnetic Films", Phys. Rev. Lett. 88, 117601 (2002).

[27] Y. Tserkovnyak, A. Brataas, and G. E. W. Bauer, "Spin pumping and magnetization dynamics in metallic multilayers", Phys. Rev. B 66, 224403 (2002).

[28] E. Saitoh, M. Ueda, H. Miyajima, and G. Tatara, "Conversion of spin current into charge current at room temperature: Inverse spin-Hall effect", Appl. Phys. Lett. 88, 182509 (2006).

[29] J.-C. Rojas-Sánchez, N. Reyren, P. Laczkowski, W. Savero, J.-P. Attané, C. Deranlot, M. Jamet, J.-M. George, L. Vila, and H. Jaffrès, "Spin Pumping and Inverse Spin Hall Effect in Platinum: The Essential Role of Spin-Memory Loss at Metallic Interfaces", Phys. Rev. Lett. 112, 106602 (2014).

[30] Y. Liu, Z. Yuan, R. J. H. Wesselink, A. A. Starikov, and P. J. Kelly, "Interface Enhancement of Gilbert Damping from First Principles", Phys. Rev. Lett. 113, 207202 (2014).

[31] F. Freimuth, S. Blügel, and Y. Mokrousov, "Direct and inverse spin-orbit torques", Phys. Rev. B 92, 064415 (2015).

[32] L. Wang, R. J. H. Wesselink, Y. Liu, Z. Yuan, K. Xia, and P. J. Kelly, "Giant Room Temperature Interface Spin Hall and Inverse Spin Hall Effects", Phys. Rev. Lett. 116, 196602 (2016).

[33] V. P. Amin and M. D. Stiles, "Spin transport at interfaces with spin-orbit coupling: Formalism”, Phys. Rev. B 94, 104419 (2016).

[34] V. P. Amin and M. D. Stiles, "Spin transport at interfaces with spin-orbit coupling: Phenomenology”, Phys. Rev. B 94, 104420 (2016).

[35] W. Zhang, W. Han, X. Jiang, S.-H. Yang, and S. S. P. Parkin, "Role of transparency of platinum-ferromagnet interfaces in determining the intrinsic magnitude of the spin Hall effect", Nature Physics 11, 496-502 (2015).

[36] M. Nguyen, D. C. Ralph, and R. A. Buhrman, "Spin Torque Study of the Spin Hall Conductivity and Spin Diffusion Length in Platinum Thin Films with Varying Resistivity", Phys. Rev. Lett. 116, 126601 (2016). 
[37] X. Tao, Q. Liu, B. Miao, R. Yu, Z. Feng, L. Sun, B. You, J. Du, K. Chen, S. Zhang, L. Zhang, Z. Yuan, D. Wu, and H. Ding, "Self-consistent determination of spin Hall angle and spin diffusion length in Pt and Pd: The role of the interface spin loss", Science Advances 4, eaat1670 (2018).

[38] A. J. Berger, E. R. J. Edwards, H. T. Nembach, O. Karis, M. Weiler, and T. J. Silva, "Determination of the spin Hall effect and the spin diffusion length of Pt from self-consistent fitting of damping enhancement and inverse spinorbit torque measurements", Phys. Rev. B 98, 024402 (2018).

[39] B. F. Miao, S. Y. Huang, D. Qu, and C. L. Chien, "Inverse Spin Hall Effect in a Ferromagnetic Metal”, Phys. Rev. Lett. 111, 066602 (2013).

[40] A. Tsukahara, Y. Ando, Y. Kitamura, H. Emoto, E. Shikoh, M. P. Delmo, T. Shinjo, and M. Shiraishi, "Self-induced inverse spin hall effect in permalloy at room temperature", Phys. Rev. B 89, 235317 (2014).

[41] K. S. Das, W. Y. Schoemaker, B. J. van Wees, and I. J. Vera-Marun, "Spin injection and detection via the anomalous spin Hall effect of a ferromagnetic metal", Phys. Rev. B 96, 220408 (2017).

[42] Y. Omori, E. Sagasta, Y. Niimi, M. Gradhand, L. E. Hueso, F. èlix Casanova, and Y. Otani, "Relation between spin hall effect and anomalous hall effect in $3 d$ ferromagnetic metals", Phys. Rev. B 99, 014403 (2019).

[43] P. Hohenberg and W. Kohn, "Inhomogeneous electron gas", Phys. Rev. 136, B864-B871 (1964).

[44] W. Kohn and L. J. Sham, "Self-consistent equations including exchange and correlation effects", Phys. Rev. 140, A1133-A1138 (1965).

[45] K. Xia, M. Zwierzycki, M. Talanana, P. J. Kelly, and G. E. W. Bauer, "Firstprinciples scattering matrices for spin-transport", Phys. Rev. B 73, 064420 (2006).

[46] A. A. Starikov, Y. Liu, Z. Yuan, and P. J. Kelly, "Calculating the transport properties of magnetic materials from first-principles including thermal and alloy disorder, non-collinearity and spin-orbit coupling", Phys. Rev. B 97, 214415 (2018).

[47] T. Ando, "Quantum point contacts in magnetic fields", Phys. Rev. B 44, 8017-8027 (1991).

[48] M. Zwierzycki, P. A. Khomyakov, A. A. Starikov, K. Xia, M. Talanana, P. X. $\mathrm{Xu}$, V. M. Karpan, I. Marushchenko, I. Turek, G. E. W. Bauer, G. Brocks, and P. J. Kelly, "Calculating scattering matrices by wave function matching", Phys. Stat. Sol. B 245, 623-640 (2008). 
[49] R. Landauer, "Electrical resistance of disordered one-dimensional lattices", Phil. Mag. 21, 863-867 (1970).

[50] O. K. Andersen, "Linear methods in band theory", Phys. Rev. B 12, 30603083 (1975).

[51] O. K. Andersen, O. Jepsen, and D. Glötzel, "Canonical description of the band structures of metals in", in Highlights of Condensed Matter Theory, edited by F. Bassani, F. Fumi, and M. P. Tosi, International School of Physics 'Enrico Fermi', Varenna, Italy, 59-176 (North-Holland, Amsterdam) (1985).

[52] H. L. Skriver, The LMTO Method: Muffin-tin Orbitals and Electronic Structure, Lecture Notes in Computer Science (Springer-Verlag) (1984).

[53] I. Turek, V. Drchal, J. Kudrnovský, M. Šob, and P. Weinberger, Electronic Structure of Disordered Alloys, Surfaces and Interfaces (Kluwer, BostonLondon-Dordrecht) (1997).

[54] P. Soven, "Coherent-potential model of substitutional disordered alloys", Phys. Rev. 156, 809-813 (1967).

[55] A. Brataas, G. E. W. Bauer, and P. J. Kelly, "Non-collinear magnetoelectronics", Phys. Rep. 427, 157-255 (2006).

[56] I. I. Mazin, "How to Define and Calculate the Degree of Spin Polarization in Ferromagnets", Phys. Rev. Lett. 83, 1427-1430 (1999).

[57] V. Vlaminck and M. Bailleul, "Current-Induced Spin-Wave Doppler Shift", Science 322, 410-413 (2008).

[58] M. Haidar and M. Bailleul, "Thickness dependence of degree of spin polarization of electrical current in permalloy thin films", Phys. Rev. B 88, 054417 (2013).

[59] M. Isasa, E. Villamor, L. E. Hueso, M. Gradhand, and F. Casanova, "Temperature dependence of spin diffusion length and spin Hall angle in Au and Pt", Phys. Rev. B 91, 024402 (2015).

[60] M. Isasa, E. Villamor, L. E. Hueso, M. Gradhand, and F. Casanova, "Erratum", Phys. Rev. B 92, 019905(E) (2015).

[61] M. Gradhand, D. V. Fedorov, P. Zahn, and I. Mertig, "Spin Hall angle versus spin diffusion length: Tailored by impurities", Phys. Rev. B 81, 245109 (2010). 
[62] S. Lowitzer, M. Gradhand, D. Ködderitzsch, D. V. Fedorov, I. Mertig, and H. Ebert, "Extrinsic and Intrinsic Contributions to the Spin Hall Effect of Alloys", Phys. Rev. Lett. 106, 056601 (2011).

[63] I. Mertig, "Transport properties of dilute alloys", Rep. Prog. Phys. 62, 237276 (1999).

[64] S. Y. Savrasov and D. Y. Savrasov, "Electron-phonon interactions and related physical properties of metals from linear-response theory", Phys. Rev. B 54, 16487-16501 (1996).

[65] P. A. Khomyakov, G. Brocks, V. Karpan, M. Zwierzycki, and P. J. Kelly, "Conductance calculations for quantum wires and interfaces: mode matching and Green functions", Phys. Rev. B 72, 035450 (2005).

[66] Y. Liu, A. A. Starikov, Z. Yuan, and P. J. Kelly, "First-principles calculations of magnetization relaxation in pure $\mathrm{Fe}$, $\mathrm{Co}$, and $\mathrm{Ni}$ with frozen thermal lattice disorder", Phys. Rev. B 84, 014412 (2011).

[67] Y. Liu, Z. Yuan, R. J. H. Wesselink, A. A. Starikov, M. van Schilfgaarde, and P. J. Kelly, "Direct method for calculating temperature-dependent transport properties", Phys. Rev. B 91, 220405(R) (2015).

[68] Z. Yuan, R. J. H. Wesselink, K. Gupta, A. N. Other, S. W. Els, and P. J. Kelly, "Calculating spin transport properties from first principles: chemical potentials", Phys. Rev. B (2019), to be published.

[69] K. M. Schep, J. B. A. N. van Hoof, P. J. Kelly, G. E. W. Bauer, and J. E. Inglesfield, "Interface resistances of magnetic multilayers", Phys. Rev. B 56, 10805-10808 (1997).

[70] K. Xia, P. J. Kelly, G. E. W. Bauer, I. Turek, J. Kudrnovský, and V. Drchal, "Interface resistance of disordered magnetic multilayers", Phys. Rev. B 63, 064407 (2001).

[71] P. X. Xu, K. Xia, M. Zwierzycki, M. Talanana, and P. J. Kelly, "OrientationDependent Transparency of Metallic Interfaces", Phys. Rev. Lett. 96, 176602 (2006).

[72] A. A. Starikov, P. J. Kelly, A. Brataas, Y. Tserkovnyak, and G. E. W. Bauer, "Unified First-Principles Study of Gilbert Damping, Spin-Flip Diffusion and Resistivity in Transition Metal Alloys", Phys. Rev. Lett. 105, 236601 (2010).

[73] D. R. Lide, ed., 90th edition (CRC Press/Taylor and Francis, Boca Raton, FL) (2009). 
[74] O. K. Andersen and O. Jepsen, "Explicit, First-Principles Tight-Binding Theory", Phys. Rev. Lett. 53, 2571-2574 (1984).

[75] O. K. Andersen, Z. Pawlowska, and O. Jepsen, "Illustration of the linearmuffin-tin-orbital tight-binding representation: Compact orbitals and charge density in Si", Phys. Rev. B 34, 5253-5269 (1986).

[76] I. Turek, J. Kudrnovský, V. Drchal, L. Szunyogh, and P. Weinberger, "Interatomic electron transport by semiempirical and ab-initio tight-binding approaches", Phys. Rev. B 65, 125101 (2002).

[77] S. Wang, Y. Xu, and K. Xia, "First-principles study of spin-transfer torques in layered systems with noncollinear magnetization", Phys. Rev. B 77, 184430 (2008).

[78] J. Shi, P. Zhang, D. Xiao, and Q. Niu, "Proper Definition of Spin Current in Spin-Orbit Coupled Systems”, Phys. Rev. Lett. 96, 076604 (2006).

[79] M. S. S. Brooks and P. J. Kelly, "Large Orbital-Moment Contribution to $5 f$ Band Magnetism”, Phys. Rev. Lett. 51, 1708-1711 (1983).

[80] G. H. O. Daalderop, P. J. Kelly, and M. F. H. Schuurmans, "First-principles calculation of the magnetocrystalline anisotropy energy of iron, cobalt and nickel", Phys. Rev. B 41, 11919-11937 (1990).

[81] C. Y. Ho, M. W. Ackerman, K. Y. Wu, T. N. Havill, R. H. Bogaard, R. A. Matula, S. G. Oh, and H. M. James, "Electrical resistivity of ten selected binary alloy systems", J. Phys. Chem. Ref. Data 12, 183-322 (1983).

[82] W. Park, D. V. Baxter, S. Steenwyk, I. Moraru, W. P. Pratt, Jr., and J. Bass, "Measurement of resistance and spin-memory loss (spin relaxation) at interfaces using sputtered current perpendicular-to-plane exchange-biased spin valves", Phys. Rev. B 62, 1178-1185 (2000).

[83] K. Eid, D. Portner, J. A. Borchers, R. Loloee, M. A. Darwish, M. Tsoi, R. D. Slater, K. V. O’Donovan, H. Kurt, W. P. Pratt, Jr., and J. Bass, "Absence of mean-free-path effects in the current-perpendicular-to-plane magnetoresistance of magnetic multilayers", Phys. Rev. B 65, 054424 (2002).

[84] G. Y. Guo, S. Murakami, T.-W. Chen, and N. Nagaosa, "Intrinsic Spin Hall Effect in Platinum: First-Principles Calculations", Phys. Rev. Lett. 100, 096401 (2008).

[85] O. K. Andersen, "Electronic structure of fcc transition metals Ir, Rh, Pt, and Pd", Phys. Rev. B 2, 883-906 (1970). 
[86] H. Kurt, R. Loloee, K. Eid, W. P. Pratt Jr., and J. Bass, "Spin-memory loss at $4.2 \mathrm{~K}$ in sputtered $\mathrm{Pd}$ and $\mathrm{Pt}$ and at $\mathrm{Pd} / \mathrm{Cu}$ and $\mathrm{Pt} / \mathrm{Cu}$ interfaces", Appl. Phys. Lett. 81, 4787-4789 (2002).

[87] S. O. Valenzuela and M. Tinkham, "Direct electronic measurement of the spin Hall effect", Nature 442, 176-179 (2006).

[88] K. Ando, S. Takahashi, K. Harii, K. Sasage, J. Ieda, S. Maekawa, and E. Saitoh, "Electric Manipulation of Spin Relaxation Using the Spin Hall Effect", Phys. Rev. Lett. 101, 036601 (2008).

[89] L. Vila, T. Kimura, and Y. Otani, "Evolution of the Spin Hall Effect in Pt Nanowires: Size and Temperature Effects", Phys. Rev. Lett. 99, 226604 (2007).

[90] A. Azevedo, L. H. Vilela-Leão, R. L. Rodríguez-Suárez, A. F. Lacerda Santos, and S. M. Rezende, "Spin pumping and anisotropic magnetoresistance voltages in magnetic bilayers: theory and experiment", Phys. Rev. B 83, 144402 (2011).

[91] L. Liu, R. A. Buhrman, and D. C. Ralph, "Review and analysis of measurements of the spin Hall effect in platinum", (2011), arXiv:1111.3702.

[92] Z. Feng, J. Hu, L. Sun, B. You, D. Wu, J. Du, W. Zhang, A. Hu, Y. Yang, D. M. Tang, B. S. Zhang, and H. F. Ding, "Spin Hall angle quantification from spin pumping and microwave photoresistance", Phys. Rev. B 85, 214423 (2012).

[93] K. Kondou, H. Sukegawa, S. Mitani, K. Tsukagoshi, and S. Kasai, "Evaluation of spin Hall angle and spin diffusion length by using spin current-induced ferromagnetic resonance", Applied Physics Express 5, 073002 (2012).

[94] H. Nakayama, K. Ando, K. Harii, T. Yoshino, R. Takahashi, Y. Kajiwara, K. Uchida, Y. Fujikawa, and E. Saitoh, "Geometry dependence on inverse spin Hall effect induced by spin pumping in $\mathrm{Ni}_{81} \mathrm{Fe}_{19} / \mathrm{Pt}$ films", Phys. Rev. B 85, 144408 (2012).

[95] M. Althammer, S. Meyer, H. Nakayama, M. Schreier, S. Altmannshofer, M. Weiler, H. Huebl, S. Geprägs, M. Opel, R. Gross, D. Meier, C. Klewe, T. Kuschel, J.-M. Schmalhorst, G. Reiss, L. Shen, A. Gupta, Y.-T. Chen, G. E. W. Bauer, E. Saitoh, and S. T. B. Goennenwein, "Quantitative study of the spin Hall magnetoresistance in ferromagnetic insulator/normal metal hybrids", Phys. Rev. B 87, 224401 (2013).

[96] W. Zhang, V. Vlaminck, J. E. Pearson, R. Divan, S. D. Bader, and A. Hoffmann, "Determination of the Pt spin diffusion length by spin-pumping and spin Hall effect", Appl. Phys. Lett. 103, 242414 (2013). 
[97] H. L. Wang, C. H. Du, Y. Pu, R. Adur, P. C. Hammel, and F. Y. Yang, "Scaling of Spin Hall Angle in $3 d$, $4 d$, and $5 d$ Metals from $\mathrm{Y}_{3} \mathrm{Fe}_{5} \mathrm{O}_{12} /$ Metal Spin Pumping", Phys. Rev. Lett. 112, 197201 (2014).

[98] A. Ganguly, K. Kondou, H. Sukegawa, S. Mitani, S. Kasai, Y. Niimi, Y. Otani, and A. Barman, "Thickness dependence of spin torque ferromagnetic resonance in $\mathrm{Co}_{75} \mathrm{Fe}_{25}$ /Pt bilayer films", Appl. Phys. Lett. 104, 072405 (2014).

[99] E. Sagasta, Y. Omori, M. Isasa, M. Gradhand, L. E. Hueso, Y. Niimi, Y. Otani, and F. Casanova, "Tuning the spin Hall effect of Pt from the moderately dirty to the superclean regime", Phys. Rev. B 94, 060412(R) (2016).

[100] C. Stamm, C. Murer, M. Berritta, J. Feng, M. Gabureac, P. M. Oppeneer, and P. Gambardella, "Magneto-Optical Detection of the Spin Hall Effect in Pt and W Thin Films", Phys. Rev. Lett. 119, 087203 (2017).

[101] T. Kimura, T. Sato, and Y. Otani, "Temperature Evolution of Spin Relaxation in a NiFe/Cu Lateral Spin Valve", Phys. Rev. Lett. 100, 066602 (2008).

[102] T. Kimura, J. Hamrle, and Y. Otani, "Estimation of spin-diffusion length from the magnitude of spin-current absorption: Multiterminal ferromagnetic/nonferromagnetic hybrid structures", Phys. Rev. B 72, 014461 (2005).

[103] E. Sagasta, Y. Omori, M. Isasa, Y. Otani, L. E. Hueso, and F. Casanova, "Spin diffusion length of Permalloy using spin absorption in lateral spin valves", Appl. Phys. Lett. 111, 082407 (2017).

[104] M. Zhu, C. L. Dennis, and R. D. McMichael, "Temperature dependence of magnetization drift velocity and current polarization in $\mathrm{Ni}_{80} \mathrm{Fe}_{20}$ by spinwave Doppler measurements", Phys. Rev. B 81, 140407 (2010).

[105] T. Tanaka, H. Kontani, M. Naito, T. Naito, D. S. Hirashima, K. Yamada, and J. Inoue, "Intrinsic spin Hall effect and orbital Hall effect in $4 d$ and $5 d$ transition metals", Phys. Rev. B 77, 165117 (2008).

[106] I. Turek, J. Kudrnovský, and V. Drchal, "Fermi sea term in the relativistic linear muffin-tin-orbital transport theory for random alloys", Phys. Rev. B 89, 064405 (2014).

[107] S. Mizukami, Y. Ando, and T. Miyazaki, "The Study on Ferromagnetic Resonance Linewidth for $\mathrm{NM} / 80 \mathrm{NiFe} / \mathrm{NM}(\mathrm{NM}=\mathrm{Cu}$, Ta, Pd and Pt) Films", Jpn. J. Appl. Phys. 40, 580-585 (2001).

[108] T. Nan, S. Emori, C. T. Boone, X. Wang, T. M. Oxholm, J. G. Jones, B. M. Howe, G. J. Brown, and N. X. Sun, "Comparison of spin-orbit torques and spin pumping across NiFe/Pt and NiFe/Cu/Pt interfaces", Phys. Rev. B 91, 214416 (2015). 
[109] T. Seki, Y. Hasegawa, S. Mitani, S. Takahashi, H. Imamura, S. Maekawa, J. Nitta, and K. Takanashi, "Giant spin hall effect in perpendicularly spinpolarized fept/au devices", Nature Materials 7, 125-129 (2008).

[110] T. Seki, I. Sugai, Y. Hasegawa, S. Mitani, and K. Takanashi, "Spin hall effect and nernst effect in fept/au multi-terminal devices with different au thicknesses", Sol. State Comm. 150, 496-499 (2010).

[111] B. Gu, I. Sugai, T. Ziman, G. Y. Guo, N. Nagaosa, T. Seki, K. Takanashi, and S. Maekawa, "Surface-assisted spin hall effect in au films with pt impurities", Phys. Rev. Lett. 105, 216401 (2010).

[112] M. B. Jungfleisch, W. Zhang, J. Sklenar, W. Jiang, J. E. Pearson, J. B. Ketterson, and A. Hoffmann, "Interface-driven spin-torque ferromagnetic resonance by rashba coupling at the interface between nonmagnetic materials", Phys. Rev. B 93, 224419 (2016).

[113] V. P. Amin, J. Zemen, and M. D. Stiles, "Interface-Generated Spin Currents", Phys. Rev. Lett. 121, 136805 (2018).

[114] C. Galinon, K. Tewolde, R. Loloee, W.-C. Chiang, S. Olson, H. Kurt, W. P. Pratt Jr., J. Bass, P. X. Xu, K. Xia, and M. Talanana, "Pd/Ag and Pd/Au interface specific resistances and interfacial spin flipping", Appl. Phys. Lett. 86, 182502 (2005).

[115] K. D. Belashchenko, A. A. Kovalev, and M. van Schilfgaarde, "Theory of Spin Loss at Metallic Interfaces", Phys. Rev. Lett. 117, 207204 (2016).

[116] K. Dolui and B. K. Nikolić, "Spin-memory loss due to spin-orbit coupling at ferromagnet/heavy-metal interfaces: Ab initio spin-density matrix approach", Phys. Rev. B 96, 220403 (2017).

[117] R. J. H. Wesselink, K. Gupta, Z. Yuan, and P. J. Kelly, "Calculating spin transport properties from first principles: Spin currents", Phys. Rev. B 99, 144409 (2019).

[118] A. Fert and S.-F. Lee, "Theory of the bipolar spin switch", Phys. Rev. B 53, 6554-6565 (1996).

[119] M. I. D'yakonov and V. I. Perel, "Possibility of orienting electrons spins with a current", JETP Lett. 13, 467-469 (1971).

[120] S. Datta, Electronic Transport in Mesoscopic Systems (Cambridge University Press, Cambridge) (1995). 
[121] S. Y. Huang, X. Fan, D. Qu, Y. P. Chen, W. G. Wang, J. Wu, T. Chen, J. Q. Xiao, and C. L. Chien, "Transport Magnetic Proximity Effects in Platinum", Phys. Rev. Lett. 109, 107204 (2012).

[122] M. Weiler, M. Althammer, M. Schreier, J. Lotze, M. Pernpeintner, S. Meyer, H. Huebl, R. Gross, A. Kamra, J. Xiao, Y.-T. Chen, H. Jiao, G. E. W. Bauer, and S. T. B. Goennenwein, "Experimental test of the spin mixing interface conductivity concept", Phys. Rev. Lett. 111, 176601 (2013).

[123] C. T. Boone, J. M. Shaw, H. T. Nembach, and T. J. Silva, "Spin-scattering rates in metallic thin films measured by ferromagnetic resonance damping enhanced by spin-pumping", J. Appl. Phys. 117, 223910 (2015).

[124] L. J. Zhu, D. C. Ralph, and R. A. Buhrman, "Irrelevance of magnetic proximity effect to spin-orbit torques in heavy-metal/ferromagnet bilayers", Phys. Rev. B 98, 134406 (2018).

[125] K. M. Schep, J. B. A. N. van Hoof, P. J. Kelly, G. E. W. Bauer, and J. E. Inglesfield, "Theory of interface resistances", J. Magn. \& Magn. Mater. 177, 1166-1167 (1998).

[126] A. Brataas, Y. V. Nazarov, and G. E. W. Bauer, "Finite-element theory of transport in ferromagnetic-normal metal systems", Phys. Rev. Lett. 84, 24812484 (2000).

[127] S. Manipatruni, D. E. Nikonov, C.-C. Lin, T. A. Gosavi, H. Liu, B. Prasad, Y.-L. Huang, E. Bonturim, R. Ramesh, and I. A. Young, "Scalable energy-efficient magnetoelectric spin-orbit logic", Nature 565, 35-42 (2018).

[128] G. E. W. Bauer, K. M. Schep, K. Xia, and P. J. Kelly, "Scattering theory of interface resistance in magnetic multilayers", J. Phys. D: Appl. Phys. 35, 2410-2414 (2002).

[129] M. D. Stiles and D. R. Penn, "Calculation of spin-dependent interface resistance", Phys. Rev. B 61, 3200-3202 (2000).

[130] M. Zwierzycki, K. Xia, P. J. Kelly, G. E. W. Bauer, and I. Turek, "Spin injection through an Fe/InAs interface", Phys. Rev. B 67, 092401 (2003).

[131] K. Xia, P. J. Kelly, G. E. W. Bauer, A. Brataas, and I. Turek, "Spin torques in ferromagnetic/normal-metal structures", Phys. Rev. B 65, 220401 (2002).

[132] M. Zwierzycki, Y. Tserkovnyak, P. J. Kelly, A. Brataas, and G. E. W. Bauer, "First-principles study of magnetization relaxation enhancement and spin transfer in thin magnetic films", Phys. Rev. B 71, 064420 (2005). 
[133] I. Turek and K. Carva, "Spin-mixing conductances of metallic and halfmetallic magnetic layers", J. Phys.: Condens. Matter 19, 365203 (2007).

[134] K. Xia, P. J. Kelly, G. E. W. Bauer, and I. Turek, "Spin-dependent transparency of ferromagnet/superconductor interfaces", Phys. Rev. Lett. 89, 166603 (2002).

[135] W. Betteridge, "The properties of metallic cobalt", Progress in Materials Science 24, 51 - 142 (1980).

[136] F. J. Lamelas, C. H. Lee, H. He, W. Vavra, and R. Clarke, "Coherent fcc stacking in epitaxial Co/Cu superlattices", Phys. Rev. B 40, 5837-5840 (1989).

[137] H. A. M. de Gronckel, K. Kopinga, W. J. M. de Jonge, P. Panissod, J. P. Schillé, and F. J. A. den Broeder, "Nanostructure of Co/Cu Multilayers", Phys. Rev. B 44, 9100-9103 (1991).

[138] Y. Tanji, "Debye Temperature and Lattice Deviation of Fe-Ni (fcc) Alloys", J. Phys. Soc. Jpn. 30, 133-138 (1971).

[139] R. J. Wakelin and E. L. Yates, "A Study of the Order-Disorder Transformation in Iron-Nickel Alloys in the Region $\mathrm{FeNi}_{3}$ ", Proc. Phys. Soc. B 66, 221-240 (1953).

[140] M. D. Kuz'min, "Shape of Temperature Dependence of Spontaneous Magnetization of Ferromagnets: Quantitative Analysis", Phys. Rev. Lett. 94, 107204 (2005).

[141] H. Masumoto, H. Saito, and M. Kikuchi, "On the magnetoresistance effect and electric resistance of single crystals of cobalt", Journal of the Japan Institute of Metals and Materials 30, 885-888 (1966), [Nippon Kinzoku GakkaiShi 30, 885 (1966)].

[142] Y. V. Sharvin, "A possible method for studying Fermi surfaces", Zh. Eksp. Teor. Fiz. 48, 984-985 (1965), [Sov. Phys. JETP 21, 655-656 (1965)].

[143] W. Zhang, M. B. Jungfleisch, W. Jiang, Y. Liu, J. E. Pearson, S. G. E. te Velthuis, A. Hoffmann, F. Freimuth, and Y. Mokrousov, "Reduced spin-Hall effects from magnetic proximity”, Phys. Rev. B 91, 115316 (2015).

[144] W. Oepts, M. A. M. Gijs, A. Reinders, R. M. Jungblut, R. M. J. van Gansewinkel, and W. J. M. de Jonge, "Perpendicular giant magnetoresistance of $\mathrm{Co} / \mathrm{Cu}$ multilayers on grooved substrates: Systematic analysis of the temperature dependence of spin-dependent scattering", Phys. Rev. B 53, 14024-14027 (1996). 
[145] F. Delille, A. Manchon, N. Strelkov, B. Dieny, M. Li, Y. Liu, P. Wang, and E. Favre-Nicolin, "Thermal variation of current perpendicular-to-plane giant magnetoresistance in laminated and nonlaminated spin valves", J. Appl. Phys. 100, 013912 (2006).

[146] P. M. Haney, H.-W. Lee, K.-J. Lee, A. Manchon, and M. D. Stiles, "Currentinduced torques and interfacial spin-orbit coupling", Phys. Rev. B 88, 214417 (2013).

[147] A. Sharma, J. A. Romero, N. Theodoropoulou, R. Loloee, W. P. Pratt, and J. Bass, "Specific resistance and scattering asymmetry of Py/Pd, Fe/V, Fe/Nb, and Co/Pt interfaces", J. Appl. Phys. 102, 113916 (2007).

[148] A. Azevedo, O. Alves Santos, R. O. Cunha, R. L. Rodríguez-Suárez, and S. M. Rezende, "Addition and subtraction of spin pumping voltages in magnetic hybrid structures", Appl. Phys. Lett. 104, 152408 (2014).

[149] H. Wang, C. Du, P. C. Hammel, and F. Yang, "Spin current and inverse spin Hall effect in ferromagnetic metals probed by $\mathrm{Y}_{3} \mathrm{Fe}_{5} \mathrm{O}_{12}$-based spin pumping", Appl. Phys. Lett. 104, 202405 (2014).

[150] C. Qin, S. Chen, Y. Cai, F. Kandaz, and Y. Ji, "Nonlocal electrical detection of spin accumulation generated by anomalous Hall effect in mesoscopic $\mathrm{Ni}_{81} \mathrm{Fe}_{19}$ films", Phys. Rev. B 96, 134418 (2017).

[151] M. Weiler, J. M. Shaw, H. T. Nembach, and T. J. Silva, "Detection of the DC Inverse Spin Hall Effect Due to Spin Pumping in a Novel Meander-Stripline Geometry", IEEE Magnetics Letters 5, 1-4 (2014).

[152] C. Du, H. Wang, F. Yang, and P. C. Hammel, "Systematic variation of spinorbit coupling with $d$-orbital filling: Large inverse spin hall effect in $3 d$ transition metals", Phys. Rev. B 90, 140407(R) (2014).

[153] J. D. Gibbons, D. MacNeill, R. A. Buhrman, and D. Ralph, "Reorientable spin direction for spin current produced by the anomalous hall effect", Phys. Rev. Appl. 9, 064033 (2018).

[154] S. Iihama, T. Taniguchi, K. Yakushiji, A. Fukushima, Y. Shiota, S. Tsunegi, R. Hiramatsu, S. Yuasa, Y. Suzuki, and H. K. title = .

[155] D. Tian, Y. Li, D. Qu, S. Y. Huang, X. Jin, and C. L. Chien, "Manipulation of pure spin current in ferromagnetic metals independent of magnetization", Phys. Rev. B 94, 020403 (2016).

[156] T. Taniguchi, J. Grollier, and M. D. Stiles, "Spin-transfer torques generated by the anomalous hall effect and anisotropic magnetoresistance", Phys. Rev. Appl. 3, 044001 (2015). 
[157] M. D. S. P. M. H. V. P. Amin, Junwen Li, "Intrinsic spin currents in ferromagnets", arXiv:1901.04022v2 (2019).

[158] Y. Ou, D. C. Ralph, and R. A. Buhrman, "Strong enhancement of the spin hall effect by spin fluctuations near the curie point of fexpt1-x alloys", Phys. Rev. Lett. 120, 097203 (2018).

[159] F. Herman and S. Skillman, Atomic Structure Calculations (Prentice Hall, Englewood Cliffs, New Jersey) (1963).

[160] D. Jo, D. Go, and H.-W. Lee, "Gigantic intrinsic orbital hall effects in weakly spin-orbit coupled metals", Phys. Rev. B 98, 214405 (2018).

[161] N. Nagaosa, J. Sinova, S. Onoda, A. H. MacDonald, and N. P. Ong, "Anomalous Hall effect", Rev. Mod. Phys. 82, 1539-1592 (2010). 


\section{Summary}

Almost everything that we know about interface transport parameters is from low temperature measurements using superconducting leads whereas the vast majority of experimental studies in spintronics are carried out at room temperature. In this thesis, I focussed on calculating spin currents at finite temperatures in bulk materials such as $\mathrm{Pt}, \mathrm{Pd}, \mathrm{Au}$, permalloy (Py) and Co and through interfaces between these materials using first-principles scattering calculations. I was able to extract bulk spin transport parameters like spin-flip diffusion lengths (SDL) and spin Hall angles (SHA) for transition metals independent of the interface contributions that are unavoidable in scattering calculations. This made it possible to establish theoretical benchmarks for bulk properties of various materials at finite temperatures. It also paved the way to disentangle interface and bulk contributions for multilayers and extract interface transport parameters such as the spin-memory loss (SML), interface resistance, interface SHA and interface spin polarization for various nonmagnetic and ferromagnetic|nonmagnetic interfaces.

In Chapter 2, I present a scheme to evaluate the spin-flip diffusion length, the spin Hall angle and the transport spin polarization for bulk metals from local spin currents. I begin by showing that interface contributions can pose a problem for determining the SDL directly from the scattering matrix. Localized currents make it possible to identify and disentangle interface-like and bulk-like transport behaviour so that my estimates of the above-mentioned bulk parameters become "interface-independent". Thermal disorder is modelled in terms of a Gaussian distribution of lattice displacements so that the resistivity I calculate for a given material at some given temperature corresponds to its experimentally reported value. To model thermal disorder in ferromagnets, account must be taken of both lattice and spin disorder. The robustness of the computational estimates for Pt and Py at room temperature is tested by varying a number of computational parameters. Considering all the variations, my estimates are found to lie within reasonable error bars.

In Chapter 3, I describe a scheme to extract the SML for nonmagnetic interfaces from local spin currents. By generalizing the Valet-Fert model along the lines of work by Baxter and coworkers [16], I derived a set of equations that describe the current discontinuity at the interface between two materials in terms of the spin memory loss and the interface resistance as well as the spin-flip diffusion lengths 
and resistivities of the two bulk materials. I evaluated the bulk parameters using the procedure developed in Chapter 2 and extracted the interface resistance from conductance calculations. These parameters, together with the values of the (discontinuous) spin current on either side of the interface are used to determine the SML.

I succeeded in determining values of the interface resistance and SML for pseudomorphic Au|Pt and Au|Pd interfaces" at room temperature. I found a "giant" interface SHA for these interfaces using the scheme developed by Wang and coworkers that led them to predict such an effect at the Py|Pt interface [32]. These findings show that spin-orbit coupling effects cannot be ignored for nonmagnetic interfaces. Another interesting observation is that the bulk SHA for Pt is found to be larger than that of Pd by only 5\%. This contradicts a widely held expectation that the SHA must depend strongly on the spin-orbit coupling strength that increases substantially on going from $3 d$ to $4 d$ to $5 d$ materials. However, the magnitude of the interface parameters I calculate for $\mathrm{Au} \mid \mathrm{Pd}$ is three-fifths of what I calculate for $\mathrm{Au} \mid \mathrm{Pt}$. Identifying the role of the SML and the interface SHA in experiments is essential to reliably and reproducibly extract values of the SHA characteristic of bulk Pd and Pt.

Finally, I studied the effect of varying the temperature and relaxing the Au lattice constant on $\mathrm{Au} \mid \mathrm{Pt}$ interface transport parameters. No temperature dependence was found within the error bars of the calculation. Constructing a fully relaxed $\mathrm{Au}$ interface with Pt requires matching differently sized unit cells of Au and Pt that are rotated with respect to each other to make them coincide. Compared to the results for the pseudomorphic interface, the interface parameters are found to be enhanced for the relaxed interface. This observation is attributed to the lack of conservation of transverse momentum and greater $\mathbf{k} \rightarrow \mathbf{k}^{\prime}$ scattering in the absence of commensurability.

Chapter 4 extends the methodology of Chapter 3 to extract values of the SML and interface spin polarization for Py|Pt and Co|Pt interfaces as a function of temperature. I first calculated the bulk spin-flip diffusion length and spin polarization (for ferromagnets) for Pt, Py and Co. Both parameters decrease with temperature for all three materials. The product of resistivity and spin-flip diffusion length for Pt is a constant as a function of temperature satisfying a relationship first proposed by Elliot and Yafet. However, it does not hold for ferromagnetic materials: for Py the product increases with temperature while for Co it decreases.

The interface resistance, SML and interface spin polarization are found to decrease monotonically with temperature for both $\mathrm{Py} \mid \mathrm{Pt}$ and $\mathrm{Co} \mid \mathrm{Pt}$; the interface spin polarization is negligibly small for both. The magnitude of the interface resistance and SML is larger for Co|Pt compared to Py|Pt. These findings indicate that chemical and spin disorder together contribute to determine the magnitude of interface

\footnotetext{
${ }^{*}$ At a pseudomorphic A|B interface, the lattice constant of material $\mathrm{A}$ is matched to that of material B
} 
parameters. Because the nonmagnetic interfaces I considered only involved lattice disorder and no chemical or spin disorder, this could be why I found no temperature dependence for Au|Pt. Finally, I also looked to see if magnetic moments induced in Pt by proximity to a ferromagnet influence my estimates. No influence of socalled proximity induced magnetization was observed for Py|Pt or Co|Pt interface parameters.

Chapter 5 deals with the discovery of a large SHA in permalloy in our calculations. This SHA is independent of the magnetization direction and does not disappear by dephasing or decrease significantly with increasing temperature. I find that this SHA can be attributed to spin-orbit splittings at the $X$ and $L$ high symmetry points close to the Fermi energy. In Chapter 3, I reported that the SHA for Pt and Pd are similar. Combining that with the findings of this chapter for a $3 d$ ferromagnetic alloy emphasizes that more work is required to understand how spin-orbit coupling contributes quantitatively to the spin Hall effect. 


\section{Acknowledgements}

When you opened this book and perhaps read it, you found a compilation of scientific findings. Apart from all the chapters written in their technical glory, it is only in the acknowledgements section where one finds due mention of all the human elements that make a thesis possible: curiosity, joy of learning, teaching and sharing knowledge, care, empathy, love, friendship and family. All the wonderful people who have been a part of my life through this $\mathrm{PhD}$ journey have helped bring together the words, ideas, questions and answers culminating into this thesis. Now I take this opportunity to express my sincere gratitude towards all of them.

Paul, you told me on my first day at CMS, "You are the driver of your own $\mathrm{PhD}$ ". It took me some time to understand your statement and to learn how to drive this vehicle myself. But you have been the fuel of this $\mathrm{PhD}$ vehicle. Your curiosity, your enthusiasm and eagerness to know more whenever I showed you a new result have been my strongest motivators. I admire your breadth of knowledge, problemsolving skills and the commitment towards making a scientific work as transparent and complete as possible in the times when "publish or perish" seems to be the mantra. I hope to imbibe these qualities in my professional life. I am indebted to you for the following and more: for being patient with me right from the beginning till the writing phase. For always keeping the door of your office open and taking out time to answer even the randomest of my questions. For your concern and consideration right from ensuring my comfortable arrival in Enschede till now in sharing advice for my future career. Andrea, I'm sorry for the times when you had to wait for Paul to come home for dinner because of our never-ending discussions and my thesis drafts. I would like to thank you for your warmth and wonderful dinners at your place.

Zhe, from our first Skype interaction in 2013, I had probably assumed you were going to be in Twente when I would arrive. We did not overlap in Twente, but yet you were always available in the last 4.5 years. A big thank you for all your detailed email and Skype explanations to my long queries. There were times when things seemed intractable, but with your calm demeanour and great suggestions, it all worked out. I am also grateful for your efforts to arrange my visits to Beijing and for Yi's and your very warm hospitality during the visits. Yi, we met in person only in 2016, but I had already learnt a great deal from you through your thesis. I am grateful for your efforts in answering my emails that sometimes required you to go 
through old calculations. You both are excellent physicists and kind supervisors. I wish you all the best for your future!

I arrived at CMS during a transition period when old members were set to leave and new members arrived halfway through my $\mathrm{PhD}$. Good colleagues are the key to a positive work environment and I was twice lucky with that. First I would like to thank the constant members of CMS. Geert, your anecdotes always liven up our corridor and coffee corner conversations. It is easy to see why you are an immensely popular teacher, I wish I would have attended one of your courses. Els, heel erg bedankt voor alles wat je doet met toewijding en efficiëntie. Ik wens je een prettige en ontspannen pensioen. Nikolai, thank you for keeping the lifeline of CMS alive and thriving.

Rien, this thesis would not have come into existence without your currents code. Thank you for guiding through all the codes during your busiest times at CMS and even afterwards via email. Despite your busy work schedule, you always took out time to send helpful detailed replies. I hope we can keep our collaboration active in the future. Diana, you took this 'feeling lost in a new country' kid under your wings and guided me through the rituals of settling into the Dutch life. I never had to ask for help because everytime you offered it even before I could ask, muchas gracias mi querido amigo por tu amor y afecto! Ireneus, it was a lot of fun to have you as my office roomie and you saved me from "deadly" office disasters. I miss both of you but we still get to reunite now and then, here and there. Nirmal and Jayita, we did not overlap for long, but you were the first ones to welcome me to CMS. You made me feel at home during a very busy time for you with newly born Rik, thank you. I'm glad to see you doing well in Bhopal. I wish you both a great academic career ahead! Mojtaba, I miss our great conversations over lunch (behind others' back!). Your deeply caring nature is admirable and I'm thankful that I can reach you at any time, any day for brotherly advice. Taher, thank you for contributing to the nice environment at CMS and for your helpful tips with mathematics problems.

Sridevi, your arrival in 2016 marked the beginning of a new phase at CMS. You were kind enough to ask me if I needed something from India even before we had met and we have kept this nice tradition more or less alive since then. I really enjoy our conversations about everything under the sun. Thank you for your care, support and motivation throughout and especially during my writing days. Rohit, it has been great working with you on different projects. I'm grateful to have you right next door to discuss with and learn from about different teeny-tiny specifics of the codes. I really admire how well you took on multiple new challenges with your $\mathrm{PhD}$. Thank you for your kind help and motivation during my writing days. Yuqiang, you make all lunch breaks brighter with your positive attitude and friendly smile. Thank you for all your help, practical advice and motivation. I wish you all the best for your thesis. Ehsan, thank you for being ever-ready to help and always making us laugh during lunch breaks.

Moving to a new country in another continent $7000 \mathrm{~km}$ away from your home 
can be exciting or overwhelming. For me it was the former, in part because of the CSER PhD 75 program. The personnel from FOM and Shell ensured that all PhDs had a great support network here in the Netherlands even before relocating. I would like to thank Joost Weber, Maria Sovago, Maria Teuwissen, Martijn de Jager from FOM and Kumar Ramachandran, Pavithra Sarma, Peter Schutjens and Frank Wubbolts from Shell. This program also gave me some great friends and my paranymphs in the Netherlands.

It is very difficult to determine the order in which I should thank my friends. Let me do it spatiotemporally. It is going to be brief because otherwise this thesis will never go to print. I met the 'Vishals' (Ahuja and Metri) on my first day at Twente and we almost immediately hit it off. Soon we met Sahana and the gang was complete. We had so many crazy and fun memories together. But more than that, I'm amazed that right on the 4th floor of Carré, I could find friends with whom I could connect on so many different levels. Sahana, you identified the artistic side in me, I had my first Dutch and swimming lessons with and probably because of you. You always gave me the best advice in times of need. Thank you for everything! Ahuja, tere sath Hindi me vaartalaap karne ka maza hi kuch aur tha. Thank you for being my google, muhavara generator and for always being there. Metri, we went from exploring Enschede market to job market together. Thank you for your Yoda gyaan and constant support.

Poorvi, our friendship grew slowly but steadily. We come from very similar social backgrounds in India, that makes us understand each other so well. I love our conversations that can go on forever. Thank you for supporting me through some of the most difficult times. Monika, initially you lured me with Swiss chocolates and aloo paranthas. Now seeing you everyday at work has turned into an essential need. Thank you for always pushing me to go out after work, motivating me to write and for your moral (and food) support. I'm also grateful that you introduced me to awk. A collective thank you to both Monika and Poorvi for agreeing to be my paranymphs. Not that you had any other choice, because you are family. I look forward to the days when we all will be done with our PhDs and can plan some exciting adventures.

Akshita, with you I finally had a place to call home in Enschede. It still remains 'hamara ghar' for me. Thank you for all your care and consideration. I miss coming back home to you and hope to see you very soon again! Vivek and Somil, we were not together at Twente but you were just a call away for anything. I'm grateful for your constant care and support and all the fun memories in Utrecht, Amsterdam and Veldhoven. Jing, thanks to Physics@Veldhoven I met you and thanks to GRC you quickly became a dear friend. We have a lot in common and you are such a great travel buddy. Thank you for your care and support. All the best with thesis writing! Can and Anna, thank you for your warmth and affection and making my Beijing visits so memorable. I fondly remember all our conversations and I hope to meet you again. 
I would like to thank all the lovely NESTers for bringing so much fun, excitement and laughter in my PhD life: Adi, Amy, Ander, Ankit, Anne, Bastien, Bram, Henk, Iris, Jessie, Jim, Karina, Kira, Louise, Manu, Marie, Natasha, Nils, Renske, Robert, Silas and Viktor. I am proud of all the plays we created and hope we stay "dramatic" all our lives. There are several other people in the Netherlands I would like to thank for various outings, trips, cooking efforts, festival celebrations, parties, being good housemates and friends: Abhistha, Alice, Biljana, Csaba, Denisa, Devashish, Harijot, Ozlem, Shantanu, Tonnishtha, Vamsi, Varghese, Vikas, Vishakha ji and Pramodji.

My friends from college and school have helped me in all possible ways to come so far. Pious, your love and faith in me always drives me to do better. Saranyo, thank you for always listening and being there for me. I cherish our friendship. Thank you Navneeta, Shilpi, Santwana, Amit, Mohanish, Akanksha, Shalini for staying in touch and constantly motivating me during my PhD. Malika, Sarang, Appu, Apoo, Abhishek, Saba, Paati, Praveena, Sujata, Jyoti thank you for your affection since school days till now! I am indebted to all my teachers from school and college who taught me valuable lessons and made me believe in myself.

Sander, in you I found not just a great friend, but also a great teacher. Thank you for your unconditional support and affection. You took me back to childhood days by reminding me of the litle things that made me happy. You taught me that with due time and patience, it is possible to learn anything. You are a great scientist and I'm very proud of you. Sietske en Joop, heel erg bedankt dat je me met open armen in je familie verwelkomt. Ik koester je genegenheid, warmte en interesse in mijn onderzoek. Ilona, Tom en iedereen in de Wildeman en Jongema familie, dank je wel voor de gezelligheid.

Last, but most importantly I would like to thank my family. Aman, you have made our family very proud. In the last 5 years, you transformed from being my younger brother into a friend whom I can turn to for advice and support. You will achieve a lot of success wherever you go. Mummy and papa, you made endless sacrifices to prioritize your children's well being and education. You gave me all the freedom and encouragement to pursue my dreams, even when the distance pained you. Papa, you always taught me to aim higher and mummy, you always taught me that there is no substitute of hardwork. This $\mathrm{PhD}$ is a result of your teachings and sacrifices. Thank you for your love and faith and everything that you do for us. I look forward to your retirement and spending more time with you in the future. 